\title{
Virtual fundamental classes for moduli spaces of sheaves on Calabi-Yau four-folds
}

\author{
Dennis Borisov and Dominic Joyce
}

\begin{abstract}
Let $\left(\boldsymbol{X}, \omega_{\boldsymbol{X}}^{*}\right)$ be a separated, -2-shifted symplectic derived $\mathbb{C}$-scheme, in the sense of Pantev, Toën, Vezzosi and Vaquié [30, of complex virtual

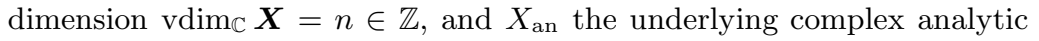
topological space. We prove that $X_{\text {an }}$ can be given the structure of a derived smooth manifold $\boldsymbol{X}_{\mathrm{dm}}$, of real virtual dimension vdim $\operatorname{dim}_{\mathbb{d m}}=n$. This $\boldsymbol{X}_{\mathrm{dm}}$ is not canonical, but is independent of choices up to bordisms fixing the underlying topological space $X_{\text {an }}$. There is a 1-1 correspondence between orientations on $\left(\boldsymbol{X}, \omega_{\boldsymbol{X}}^{*}\right)$ and orientations on $\boldsymbol{X}_{\mathrm{dm}}$.

Because compact, oriented derived manifolds have virtual classes, this means that proper, oriented -2 -shifted symplectic derived $\mathbb{C}$-schemes have virtual classes, in either homology or bordism. This is surprising, as conventional algebro-geometric virtual cycle methods fail in this case. Our virtual classes have half the expected dimension.

Now derived moduli schemes of coherent sheaves on a Calabi-Yau 4fold are expected to be -2 -shifted symplectic (this holds for stacks). We propose to use our virtual classes to define new Donaldson-Thomas style invariants 'counting' (semi)stable coherent sheaves on Calabi-Yau 4-folds $Y$ over $\mathbb{C}$, which should be unchanged under deformations of $Y$.
\end{abstract}

\section{Contents}

1 Introduction $\quad 2$

2 Background material 4

2.1 Commutative differential graded algebras . . . . . . . . . . 5

2.2 Derived algebraic geometry and derived schemes . . . . . . . 7

2.3 PTVV's shifted symplectic geometry . . . . . . . . . . . 8

2.4 Orientations on $k$-shifted symplectic derived schemes . . . . . . 11

2.5 Kuranishi atlases . . . . . . . . . . . . . . . . . . 12

2.6 Derived smooth manifolds and virtual classes . . . . . . . . 15

3 The main results $\quad 19$

3.1 Zariski homotopy atlases on derived schemes . . . . . . . . . 20

3.2 Interpreting Zariski atlases using complex geometry . . . . . . . 21

3.3 Subbundles $E^{-} \subseteq E$ and Kuranishi neighbourhoods $\ldots \ldots .27$ 
3.4 Comparing $\left(U_{J}, E_{J}^{-}\right),\left(U_{K}, E_{K}^{-}\right)$under $\Phi_{J K} \ldots \ldots \ldots \ldots$

3.5 Constructing Kuranishi atlases and derived manifolds . . . . . . 31

3.6 Orientations, bordism classes, and virtual classes . . . . . . . . . 34

3.7 Working relative to a smooth base $\mathbb{C}$-scheme $Z \ldots \ldots$. . . . . 34

3.8 'Holomorphic Donaldson invariants' of Calabi-Yau 4-folds . . . . 40

3.9 Motivation from gauge theory, and 'SU(4) instantons' . . . . . . 42

4 Proof of Theorem $3.1 \quad 44$

5 Proof of Theorem $3.7 \quad 51$

5.1 Theorem 3.7(a): $(*)$ is an open condition $\ldots \ldots \ldots \ldots \ldots$

5.2 Theorem 3.7 (b): extending pairs $\left(U, E^{-}\right)$satisfying $(*) \ldots \ldots .53$

5.3 Theorem 3.7 (c): $s^{-1}(0)=\left(s^{+}\right)^{-1}(0)$ locally in $U \ldots \ldots \ldots$

6 Proofs of some auxiliary results 5

6.1 Proof of Proposition $3.13 \ldots \ldots \ldots$

6.2 Proof of Proposition $3.14 \ldots \ldots \ldots$

6.3 Proof of Proposition $3.17 \ldots \ldots \ldots$

6.4 Proof of Proposition $3.18 \ldots \ldots \ldots \ldots$

References $\quad 67$

\section{Introduction}

This paper will relate two apparently rather different classes of 'derived' geometric spaces. The first class is derived $\mathbb{C}$-schemes $\boldsymbol{X}$, in the Derived Algebraic Geometry of Toën and Vezzosi [33, 35], equipped with a -2-shifted symplectic structure $\omega_{\boldsymbol{X}}^{*}$ in the sense of Pantev, Toën, Vaquié and Vezzosi [30]. Such $\left(\boldsymbol{X}, \omega_{\boldsymbol{X}}^{*}\right)$ are the expected structure on 4 -Calabi-Yau derived moduli $\mathbb{C}$-schemes.

The second class is derived smooth manifolds $\boldsymbol{X}_{\mathrm{dm}}$, in Derived Differential Geometry. There are several different models available: the derived manifolds of Spivak [31] and Borisov-Noël [6, 7], (which form $\infty$-categories DerMan $\mathbf{S p i}$, $\operatorname{DerMan}_{\mathrm{BoNo}}$ ), and the second author's d-manifolds [18 20] (a strict 2-category dMan), and m-Kuranishi spaces [22, §4.7] (a weak 2-category mKur).

As it is known that equivalence classes of objects in all these higher categories are in natural bijection, these four models are interchangeable for our purposes. But we use theorems proved for d-manifolds or (m-)Kuranishi spaces.

Here is a summary of our main results, taken from Theorems 3.15, 3.16 and 3.24 and Propositions 3.17 and 3.18 below.

Theorem 1.1. Let $\left(\boldsymbol{X}, \omega_{\boldsymbol{X}}^{*}\right)$ be a-2-shifted symplectic derived $\mathbb{C}$-scheme, in the sense of Pantev et al. [30], with complex virtual dimension $\operatorname{vdim}_{\mathbb{C}} \boldsymbol{X}=n$ in $\mathbb{Z}$, and write $X_{\text {an }}$ for the set of $\mathbb{C}$-points of $X=t_{0}(\boldsymbol{X})$, with the complex analytic topology. Suppose that $X$ is separated, and $X_{\text {an }}$ is second countable. Then we can make the topological space $X_{\mathrm{an}}$ into a derived manifold $\boldsymbol{X}_{\mathrm{dm}}$ of real virtual dimension $\operatorname{vdim}_{\mathbb{R}} \boldsymbol{X}_{\mathrm{dm}}=n$, in the sense of any of [6,7, 18, 20, 22, 31]. 
There is a natural 1-1 correspondence between orientations on $\left(\boldsymbol{X}, \omega_{\boldsymbol{X}}^{*}\right)$, in the sense of 2.4 , and orientations on $\boldsymbol{X}_{\mathrm{dm}}$, in the sense of 2.6

The (oriented) derived manifold $\boldsymbol{X}_{\mathrm{dm}}$ above depends on arbitrary choices made in its construction. However, $\boldsymbol{X}_{\mathrm{dm}}$ is independent of choices up to (oriented) bordisms of derived manifolds which fix the underlying topological space.

All the above extends to (oriented) -2-shifted symplectic derived schemes $\left(\boldsymbol{\pi}: \boldsymbol{X} \rightarrow Z, \omega_{\boldsymbol{X} / Z}^{*}\right)$ over a base $Z$ which is a smooth affine $\mathbb{C}$-scheme of pure dimension, yielding an (oriented) derived manifold $\boldsymbol{\pi}_{\mathrm{dm}}: \boldsymbol{X}_{\mathrm{dm}} \rightarrow Z_{\mathrm{an}}$ over the complex manifold $Z_{\mathrm{an}}$ associated to $Z$, regarded as an (oriented) real manifold.

In $\$ 2.5$ we give a short definition of Kuranishi atlases $\mathcal{K}$ on a topological space $X$. These are families of 'Kuranishi neighbourhoods' $(V, E, s, \psi)$ on $X$ and 'coordinate changes' between them, based on work of Fukaya, Oh, Ohta and Ono [14,15] in symplectic geometry. The hard work in proving Theorem 1.1 is using $\left(\boldsymbol{X}, \omega_{\boldsymbol{X}}^{*}\right)$ to construct a Kuranishi atlas $\mathcal{K}$ on $X_{\text {an }}$. Then we use results from [6, 7, 18, 20,22] to convert $\left(X_{\mathrm{an}}, \mathcal{K}\right)$ into a derived manifold $\boldsymbol{X}_{\mathrm{dm}}$.

Readers of this papers do not need to understand derived manifolds, if they do not want to. They can just think in terms of Kuranishi atlases, as is common in symplectic geometry, without passing to derived manifolds.

We prove Theorem 1.1 using a 'Darboux Theorem' for $k$-shifted symplectic derived schemes by Bussi, Brav and the second author [4. This paper is related to the series [2 5, 21, mostly concerning the -1-shifted (3-Calabi-Yau) case.

An important motivation for proving Theorem 1.1 is that compact, oriented derived manifolds have virtual classes, in both bordism and homology. As in 33.6 3.7. from Theorem 1.1 we may deduce:

Corollary 1.2. Let $\left(\boldsymbol{X}, \omega_{\boldsymbol{X}}^{*}\right)$ be a proper, oriented -2-shifted symplectic derived $\mathbb{C}$-scheme, with $\operatorname{vdim}_{\mathbb{C}} \boldsymbol{X}=n$. Theorem 1.1 gives a compact, oriented derived manifold $\boldsymbol{X}_{\mathrm{dm}}$ with $\operatorname{vdim}_{\mathbb{R}} \boldsymbol{X}_{\mathrm{dm}}=n$. We may define a $\boldsymbol{d}$-bordism class $\left[\boldsymbol{X}_{\mathrm{dm}}\right]_{\mathrm{dbo}}$ in the bordism group $B_{n}(*)$, and a virtual class $\left[\boldsymbol{X}_{\mathrm{dm}}\right]_{\mathrm{virt}}$ in the homology group $H_{n}\left(X_{\mathrm{an}} ; \mathbb{Z}\right)$, depending only on $\left(\boldsymbol{X}, \omega_{\boldsymbol{X}}^{*}\right)$ and its orientation.

Let $\boldsymbol{X}$ be a derived $\mathbb{C}$-scheme, $Z$ a connected $\mathbb{C}$-scheme, $\boldsymbol{\pi}: \boldsymbol{X} \rightarrow Z$ be proper, and $\left[\omega_{\boldsymbol{X} / Z}\right]$ a family of oriented -2 -shifted symplectic structures on $\boldsymbol{X} / Z$, with $\operatorname{vdim}_{\mathbb{C}} \boldsymbol{X} / Z=n$. For each $z \in Z_{\text {an }}$ we have a proper, oriented -2 shifted symplectic $\mathbb{C}$-scheme $\left(\boldsymbol{X}^{z}, \omega_{\boldsymbol{X}^{z}}^{*}\right)$ with $\operatorname{vdim} \boldsymbol{X}^{z}=n$. Then $\left[\boldsymbol{X}_{\mathrm{dm}}^{z_{1}}\right]_{\mathrm{dbo}}$ $=\left[\boldsymbol{X}_{\mathrm{dm}}^{z_{2}}\right]_{\mathrm{dbo}}$ and $\imath_{*}^{z_{1}}\left(\left[\boldsymbol{X}_{\mathrm{dm}}^{z_{1}}\right]_{\text {virt }}\right)=\imath_{*}^{z_{2}}\left(\left[\boldsymbol{X}_{\mathrm{dm}}^{z_{2}}\right]_{\text {virt }}\right)$ for all $z_{1}, z_{2} \in Z_{\mathrm{an}}$, with $\imath_{*}^{z}\left(\left[\boldsymbol{X}_{\mathrm{dm}}^{z}\right]_{\mathrm{virt}}\right) \in H_{n}\left(X_{\mathrm{an}} ; \mathbb{Z}\right)$ the pushforward under the inclusion $\imath^{z}: X_{\mathrm{an}}^{z} \hookrightarrow X_{\mathrm{an}}$.

So, proper, oriented -2 -shifted symplectic derived $\mathbb{C}$-schemes $\left(\boldsymbol{X}, \omega_{\boldsymbol{X}}^{*}\right)$ have virtual classes. This is not obvious, in fact it is rather surprising. Firstly, if $\left(\boldsymbol{X}, \omega_{\boldsymbol{X}}^{*}\right)$ is -2 -shifted symplectic then $X=t_{0}(\boldsymbol{X})$ has a natural obstruction theory $\left.\mathbb{L}_{\boldsymbol{X}}\right|_{X} \rightarrow \mathbb{L}_{X}$ in the sense of Behrend and Fantechi [1, which is perfect in the interval $[-2,0]$. But the Behrend-Fantechi construction of virtual cycles [1] works only for obstruction theories perfect in $[-1,0]$, and does not apply here.

Secondly, our virtual cycle has real dimension $\operatorname{vdim}_{\mathbb{C}} \boldsymbol{X}=\frac{1}{2} \operatorname{vdim}_{\mathbb{R}} \boldsymbol{X}$, which is half what we might have expected. A heuristic explanation is that one should be able to make $\boldsymbol{X}$ into a 'derived $C^{\infty}$-scheme' $\boldsymbol{X}^{C^{\infty}}$ (not a derived manifold), in 
some sense similar to Lurie [26, §4.5] or Spivak [31], and $\left(\boldsymbol{X}^{C^{\infty}}, \operatorname{Im} \omega_{\boldsymbol{X}}^{*}\right)$ should be a 'real -2-shifted symplectic derived $C^{\infty}$-scheme', with $\operatorname{Im} \omega_{\boldsymbol{X}}^{*}$ the imaginary part of $\omega_{\boldsymbol{X}}^{*}$. There should be a morphism $\boldsymbol{X}^{C^{\infty}} \rightarrow \boldsymbol{X}_{\mathrm{dm}}$ which is a 'Lagrangian fibration' of $\left(\boldsymbol{X}^{C^{\infty}}, \operatorname{Im} \omega_{\boldsymbol{X}}^{*}\right)$. So $\operatorname{vdim}_{\mathbb{R}} \boldsymbol{X}_{\mathrm{dm}}=\frac{1}{2} \operatorname{vdim}_{\mathbb{R}} \boldsymbol{X}^{C^{\infty}}=\frac{1}{2} \operatorname{vdim}_{\mathbb{R}} \boldsymbol{X}$, as for Lagrangian fibrations $\pi:(S, \omega) \rightarrow B$ we have $\operatorname{dim} B=\frac{1}{2} \operatorname{dim} S$.

The main application we intend for these results, motivated by Donaldson and Thomas [13] and explained in $\lceil 3.8-\$ 3.9$, is to define new invariants 'counting' (semi)stable coherent sheaves on Calabi-Yau 4-folds $Y$ over $\mathbb{C}$, which should be unchanged under deformations of $Y$. These are similar to Donaldson-Thomas invariants [24, 25, 32] 'counting' (semi)stable coherent sheaves of Calabi-Yau 3 -folds, and could be called 'holomorphic Donaldson invariants', as they are complex analogues of Donaldson invariants of 4-manifolds [12].

Pantev, Toën, Vaquié and Vezzosi [30, §2.1] show that any derived moduli stack $\mathcal{M}$ of coherent sheaves (or complexes of coherent sheaves) on a CalabiYau $m$-fold has a $(2-m)$-shifted symplectic structure $\omega_{\mathcal{M}}^{*}$, so in particular 4-Calabi-Yau moduli stacks are -2-shifted symplectic. Given an analogue of this for derived moduli schemes, and a way to define orientations upon them, Corollary 1.2 would give virtual classes for moduli schemes of (semi)stable coherent sheaves on Calabi-Yau 4-folds, and so enable us to define invariants.

It is well known that there is a great deal of interesting and special geometry, related to String Theory, concerning Calabi-Yau 3-folds and 3-Calabi-Yau categories - Mirror Symmetry, Donaldson-Thomas theory, and so on. One message of this paper is that there should also be special geometry concerning Calabi-Yau 4-folds and 4-Calabi-Yau categories, which is not yet understood.

During the writing of this paper, Cao and Leung [8, 10] also proposed a theory of invariants counting coherent sheaves on Calabi-Yau 4-folds, based on gauge theory rather than derived geometry. We discuss their work in 3.9 .

Section 2 provides background material on derived schemes, shifted symplectic structures upon them, Kuranishi atlases, and derived manifolds. The heart of the paper is $\$ 3$, with the definitions, main results, shorter proofs, and discussion. Longer proofs of results in $\$ 3$ are deferred to sections 46 .

Acknowledgements. We would like to thank Yalong Cao, Conan Leung, Bertrand Toën, Gabriele Vezzosi, and a referee for helpful conversations. This research was supported by EPSRC Programme Grant EP/I033343/1.

\section{Background material}

We begin with some background material and notation needed later. Some references are Toën and Vezzosi [33 35] for $2.1-\$ 2.2$, Pantev, Toën, Vezzosi and Vaquié [30] and Brav, Bussi and Joyce [4] for \$2.3] and Spivak [31, Borisov and Noël [6, 7] and Joyce [18, 20, 22, for $\$ 2.6$. 


\subsection{Commutative differential graded algebras}

Definition 2.1. Write cdga $\mathbf{C}_{\mathbb{C}}$ for the category of commutative differential graded $\mathbb{C}$-algebras in nonpositive degrees, and cdga $_{\mathbb{C}}^{\text {op }}$ for its opposite category. In fact $\mathbf{c d g a}_{\mathbb{C}}$ has the additional structure of a model category (a kind of $\infty$-category), but we only use this in the proof of Theorem 3.1 in 44 In the rest of the paper we treat $\mathbf{c d g a}_{\mathbb{C}}, \mathbf{c d g a}_{\mathbb{C}}^{\text {op }}$ just as ordinary categories.

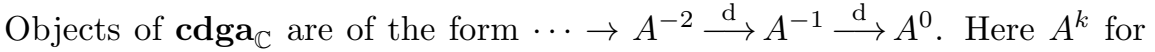
$k=0,-1,-2, \ldots$ is the $\mathbb{C}$-vector space of degree $k$ elements of $A$, and we have a $\mathbb{C}$-bilinear, associative, supercommutative multiplication $\cdot: A^{k} \times A^{l} \rightarrow A^{k+l}$ for $k, l \leqslant 0$, an identity $1 \in A^{0}$, and differentials d : $A^{k} \rightarrow A^{k+1}$ for $k<0$ satisfying

$$
\mathrm{d}(a \cdot b)=(\mathrm{d} a) \cdot b+(-1)^{k} a \cdot(\mathrm{d} b)
$$

for all $a \in A^{k}, b \in A^{l}$. We write such objects as $A^{\bullet}$ or $\left(A^{*}, \mathrm{~d}\right)$.

Here and throughout we will use the superscript " * ' to denote graded objects (e.g. graded algebras or vector spaces), where $*$ stands for an index in $\mathbb{Z}$, so that $A^{*}$ means $\left(A^{k}, k \in \mathbb{Z}\right)$. We will use the superscript ' $\bullet$ ' to denote differential graded objects (e.g. differential graded algebras or complexes), so that $A^{\bullet}$ means $\left(A^{*}, \mathrm{~d}\right)$, the graded object $A^{*}$ together with the differential d.

Morphisms $\alpha: A^{\bullet} \rightarrow B^{\bullet}$ in cdga $_{\mathbb{C}}$ are $\mathbb{C}$-linear maps $\alpha^{k}: A^{k} \rightarrow B^{k}$ for all $k \leqslant 0$ commuting with all the structures on $A^{\bullet}, B^{\bullet}$.

A morphism $\alpha: A^{\bullet} \rightarrow B^{\bullet}$ is a quasi-isomorphism if $H^{k}(\alpha): H^{k}\left(A^{\bullet}\right) \rightarrow$ $H^{k}\left(B^{\bullet}\right)$ is an isomorphism on cohomology groups for all $k \leqslant 0$. A fundamental principle of derived algebraic geometry is that $\mathbf{c d g a}_{\mathbb{C}}$ is not really the right category to work in, but instead one wants to define a new category (or better, $\infty$-category) by inverting (localizing) quasi-isomorphisms in $\mathbf{c d g a}_{\mathbb{C}}$.

We will call $A^{\bullet} \in \mathbf{c d g a}_{\mathbb{C}}$ of standard form if $A^{0}$ is a smooth finitely generated $\mathbb{C}$-algebra of pure dimension, and the graded $\mathbb{C}$-algebra $A^{*}$ is freely generated over $A^{0}$ by finitely many generators in each degree $i=-1,-2, \ldots$. Here we require $A^{0}$ to be smooth of pure dimension so that $\left(\operatorname{Spec} A^{0}\right)_{\text {an }}$ is a complex manifold, rather than a disjoint union of complex manifolds of different dimensions. This is not crucial, but will be convenient in 33 .

Remark 2.2. Brav, Bussi and Joyce [4, Def. 2.9] work with a stronger notion of standard form cdgas than us, as they require $A^{*}$ to be freely generated over $A^{0}$ by finitely many generators, all in negative degrees. In contrast, we allow infinitely many generators, but only finitely many in each degree $i=-1,-2, \ldots$.

The important thing for us is that since standard form cdgas in the sense of [4 are also standard form in the (slightly weaker) sense of this paper, we can apply some of their results [4, Th.s 4.1, 4.2, 5.18] on the existence and properties of nice standard form cdga local models for derived schemes.

Definition 2.3. Let $A^{\bullet} \in \mathbf{c d g a}_{\mathbb{C}}$, and write $D(\bmod A)$ for the derived category of dg-modules over $A^{\bullet}$. Define a derivation of degree $k$ from $A^{\bullet}$ to an $A^{\bullet}$-module $M^{\bullet}$ to be a $\mathbb{C}$-linear map $\delta: A^{\bullet} \rightarrow M^{\bullet}$ that is homogeneous of degree $k$ with

$$
\delta(f g)=\delta(f) g+(-1)^{k|f|} f \delta(g) .
$$


Just as for ordinary commutative algebras, there is a universal derivation into an $A^{\bullet}$-module of Kähler differentials $\Omega_{A}^{1} \bullet$, which can be constructed as $I / I^{2}$ for $I=\operatorname{Ker}\left(m: A^{\bullet} \otimes A^{\bullet} \rightarrow A^{\bullet}\right)$. The universal derivation $\delta: A^{\bullet} \rightarrow \Omega_{A}^{1}{ }^{\bullet}$ is $\delta(a)=a \otimes 1-1 \otimes a \in I / I^{2}$. One checks that $\delta$ is a universal degree 0 derivation, so that $\circ \delta: \operatorname{Hom}_{A}^{\bullet}\left(\Omega_{A}^{1} \bullet, M^{\bullet}\right) \rightarrow \operatorname{Der}^{\bullet}\left(A, M^{\bullet}\right)$ is an isomorphism of dg-modules.

Note that $\Omega_{A \bullet}^{1}=\left(\left(\Omega_{A}^{1} \bullet\right)^{*}, \mathrm{~d}\right)$ is canonical up to strict isomorphism, not just up to quasi-isomorphism of complexes, or up to equivalence in $D(\bmod A)$. Also, the underlying graded vector space $\left(\Omega_{A}^{1} \bullet\right)^{*}$, as a module over the graded algebra $A^{*}$, depends only on $A^{*}$ and not on the differential d in $A^{\bullet}=\left(A^{*}, \mathrm{~d}\right)$.

Similarly, given a morphism of cdgas $\Phi: A^{\bullet} \rightarrow B^{\bullet}$, we can define the relative Kähler differentials $\Omega_{B}^{1} \bullet / A^{\bullet}$.

The cotangent complex $\mathbb{L}_{A} \bullet$ of $A^{\bullet}$ is related to the Kähler differentials $\Omega_{A}^{1} \bullet$, but is not quite the same. If $\Phi: A^{\bullet} \rightarrow B^{\bullet}$ is a quasi-isomorphism of cdgas over $\mathbb{C}$, then $\Phi_{*}: \Omega_{A}^{1} \bullet \otimes_{A} \bullet B^{\bullet} \rightarrow \Omega_{B}^{1} \bullet$ may not be a quasi-isomorphism of $B^{\bullet}$-modules. So Kähler differentials are not well-behaved under localizing quasi-isomorphisms of cdgas, which is bad for doing derived algebraic geometry.

The cotangent complex $\mathbb{L}_{A}$ • is a substitute for $\Omega_{A}^{1}$. which is well-behaved under localizing quasi-isomorphisms. It is an object in $D(\bmod A)$, canonical up to equivalence. We can define it by replacing $A^{\bullet}$ by a quasi-isomorphic, cofibrant (in the sense of model categories) cdga $B^{\bullet}$, and then setting $\mathbb{L}_{A} \bullet$ $\left(\Omega_{B^{\bullet}}^{1}\right) \otimes_{B^{\bullet}} A^{\bullet}$. We will be interested in the $p^{\text {th }}$ exterior power $\Lambda^{p} \mathbb{L}_{A^{\bullet}}$, and the dual $\left(\mathbb{L}_{A} \bullet\right)^{\vee}$, which is called the tangent complex, and written $\mathbb{T}_{A} \bullet=\left(\mathbb{L}_{A} \bullet\right)^{\vee}$.

There is a de Rham differential $\mathrm{d}_{\mathrm{dR}}: \Lambda^{p} \mathbb{L}_{A} \bullet \rightarrow \Lambda^{p+1} \mathbb{L}_{A^{\bullet}}$, a morphism of complexes, with $\mathrm{d}_{\mathrm{dR}}^{2}=0: \Lambda^{p} \mathbb{L}_{A} \bullet \rightarrow \Lambda^{p+2} \mathbb{L}_{A} \bullet$. Note that each $\Lambda^{p} \mathbb{L}_{A} \bullet$ is also a complex with its own internal differential $\mathrm{d}:\left(\Lambda^{p} \mathbb{L}_{A} \bullet\right)^{k} \rightarrow\left(\Lambda^{p} \mathbb{L}_{A} \bullet\right)^{k+1}$, and $\mathrm{d}_{\mathrm{dR}}$ being a morphism of complexes means that $\mathrm{d} \circ \mathrm{d}_{\mathrm{dR}}=\mathrm{d}_{\mathrm{dR}} \circ \mathrm{d}$.

Similarly, given a morphism of cdgas $\Phi: A^{\bullet} \rightarrow B^{\bullet}$, we can define the relative cotangent complex $\mathbb{L}_{B \cdot / A} \bullet$

As in [4, §2.3], an important property of our standard form cdgas $A^{\bullet}$ in Definition 2.1 is that they are sufficiently cofibrant that the Kähler differentials $\Omega_{A}^{1} \bullet$ provide a model for the cotangent complex $\mathbb{L}_{A} \bullet$, so we can take $\Omega_{A}^{1} \bullet=\mathbb{L}_{A} \bullet$, without having to replace $A^{\bullet}$ by an unknown cdga $B^{\bullet}$. Thus standard form cdgas are convenient for doing explicit computations with cotangent complexes.

A morphism $\Phi: A^{\bullet} \rightarrow B^{\bullet}$ of cdgas will be called quasi-free if $\Phi^{0}: A^{0} \rightarrow B^{0}$ is a smooth morphism of $\mathbb{C}$-algebras of pure relative dimension, and as a graded $A^{*} \otimes_{A^{0}} B^{0}$-algebra $B^{*}$ is free and finitely generated in each degree. Here if $A^{\bullet}$ is of standard form and $\Phi$ is quasi-free then $B^{\bullet}$ is of standard form, and a cdga $A^{\bullet}$ is of standard form if and only if the unique morphism $\mathbb{C} \rightarrow A^{\bullet}$ is quasi-free. We will only consider quasi-free morphisms when $A^{\bullet}, B^{\bullet}$ are of standard form.

If $\Phi: A^{\bullet} \rightarrow B^{\bullet}$ is a quasi-free morphism then the relative Kähler differentials $\Omega_{B}^{1} \bullet / A \bullet$ are a model for the relative cotangent complex $\mathbb{L}_{B} \bullet / A \bullet$, so we can take $\Omega_{B}^{1} \bullet / A \bullet=\mathbb{L}_{B} \bullet / A^{\bullet}$. Thus quasi-free morphisms are a convenient class of morphisms for doing explicit computations with cotangent complexes. 


\subsection{Derived algebraic geometry and derived schemes}

Definition 2.4. Write $\mathbf{d S t}_{\mathbb{C}}$ for the $\infty$-category of derived $\mathbb{C}$-stacks (or $D^{-}$stacks) defined by Toën and Vezzosi [35, Def. 2.2.2.14], 33, Def. 4.2]. Objects $\boldsymbol{X}$ in $\mathbf{d S t}_{\mathbb{C}}$ are $\infty$-functors

$$
\boldsymbol{X}:\{\text { simplicial commutative } \mathbb{C} \text {-algebras }\} \longrightarrow\{\text { simplicial sets }\}
$$

satisfying sheaf-type conditions. There is a spectrum functor

$$
\text { Spec }: \operatorname{cdga}_{\mathbb{C}}^{\text {op }} \longrightarrow \text { dSt }_{\mathbb{C}} .
$$

A derived $\mathbb{C}$-stack $\boldsymbol{X}$ is called an affine derived $\mathbb{C}$-scheme if $\boldsymbol{X}$ is equivalent in $\mathbf{d S t}_{\mathbb{C}}$ to $\mathbf{S p e c} A^{\bullet}$ for some cdga $A^{\bullet}$ over $\mathbb{C}$. As in [33, §4.2], a derived $\mathbb{C}$-stack $\boldsymbol{X}$ is called a derived $\mathbb{C}$-scheme if it may be covered by Zariski open $\boldsymbol{Y} \subseteq \boldsymbol{X}$ with $\boldsymbol{Y}$ an affine derived $\mathbb{C}$-scheme. Write $\mathbf{d S c h}_{\mathbb{C}}$ for the full $\infty$-subcategory of

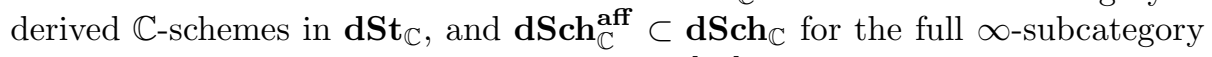
of affine derived $\mathbb{C}$-schemes. See also Toën 34 for a different but equivalent way to define derived $\mathbb{C}$-schemes, as an $\infty$-category of derived ringed spaces.

We shall assume throughout this paper that all derived $\mathbb{C}$-schemes $\boldsymbol{X}$ are locally finitely presented in the sense of Toën and Vezzosi [35, Def. 1.3.6.4]. Note that this is a strong condition, for instance it implies that the cotangent complex $\mathbb{L}_{\boldsymbol{X}}$ is perfect [35, Prop. 2.2.2.4]. A locally finitely presented classical $\mathbb{C}$-scheme $X$ need not be locally finitely presented as a derived $\mathbb{C}$-scheme. A local normal form for locally finitely presented derived $\mathbb{C}$-schemes is given in [4, Th. 4.1].

There is a classical truncation functor $t_{0}: \mathbf{d S c h}_{\mathbb{C}} \rightarrow \mathbf{S c h}_{\mathbb{C}}$ taking a derived $\mathbb{C}$-scheme $\boldsymbol{X}$ to the underlying classical $\mathbb{C}$-scheme $X=t_{0}(\boldsymbol{X})$. On affine derived schemes $\mathbf{d S c h} \mathbb{C}_{\mathbb{C}}^{\text {aff }}$ this maps $t_{0}: \operatorname{Spec} A^{\bullet} \rightarrow \operatorname{Spec} H^{0}\left(A^{\bullet}\right)=\operatorname{Spec}\left(A^{0} / \mathrm{d}\left(A^{-1}\right)\right)$.

Toën and Vezzosi show that a derived $\mathbb{C}$-scheme $\boldsymbol{X}$ has a cotangent complex $\mathbb{L}_{\boldsymbol{X}}[35, \S 1.4],[33, \S 4.2 .4-\S 4.2 .5]$ in a stable $\infty$-category $L_{\mathrm{qcoh}}(\boldsymbol{X})$ defined in [33, $\S 3.1 .7, \S 4.2 .4]$. We will be interested in the $p^{\text {th }}$ exterior power $\Lambda^{p} \mathbb{L}_{\boldsymbol{X}}$, and the dual $\left(\mathbb{L}_{\boldsymbol{X}}\right)^{\vee}$, which is called the tangent complex $\mathbb{T}_{\boldsymbol{X}}$. There is a de Rham differential $\mathrm{d}_{\mathrm{dR}}: \Lambda^{p} \mathbb{L}_{\boldsymbol{X}} \rightarrow \Lambda^{p+1} \mathbb{L}_{\boldsymbol{X}}$.

Restricted to the classical scheme $X=t_{0}(\boldsymbol{X})$, the cotangent complex $\left.\mathbb{L}_{\boldsymbol{X}}\right|_{X}$ may Zariski locally be modelled as a finite complex of vector bundles $\left[F^{-m} \rightarrow\right.$ $\left.F^{1-m} \rightarrow \cdots \rightarrow F^{0}\right]$ on $X$ in degrees $[-m, 0]$ for some $m \geqslant 0$. The (complex) virtual dimension $\operatorname{vdim}_{\mathbb{C}} \boldsymbol{X}$ is $\operatorname{vdim}_{\mathbb{C}} \boldsymbol{X}=\sum_{i=0}^{m}(-1)^{i} \operatorname{rank} F^{-i}$. It is a locally constant function $\operatorname{vdim}_{\mathbb{C}} \boldsymbol{X}: X \rightarrow \mathbb{Z}$, so is constant on each connected component of $X$. We say that $\boldsymbol{X}$ has (complex) virtual dimension $n \in \mathbb{Z}$ if $\operatorname{vdim}_{\mathbb{C}} \boldsymbol{X}=n$.

When $\boldsymbol{X}=X$ is a classical scheme, the homotopy category of $L_{\mathrm{qcoh}}(\boldsymbol{X})$ is the triangulated category $D_{\text {qcoh }}(X)$ of complexes of quasicoherent sheaves. These $\mathbb{L}_{\boldsymbol{X}}, \mathbb{T}_{\boldsymbol{X}}$ have the usual properties of (co)tangent complexes. For instance,

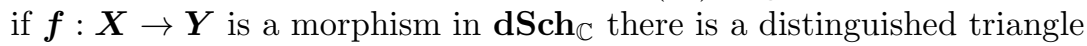

$$
f^{*}\left(\mathbb{L}_{\boldsymbol{Y}}\right) \stackrel{\mathbb{L}_{f}}{\longrightarrow} \mathbb{L}_{\boldsymbol{X}} \longrightarrow \mathbb{L}_{\boldsymbol{X} / \boldsymbol{Y}} \longrightarrow \boldsymbol{f}^{*}\left(\mathbb{L}_{\boldsymbol{Y}}\right)[1],
$$

where $\mathbb{L}_{\boldsymbol{X} / \boldsymbol{Y}}$ is the relative cotangent complex of $\boldsymbol{f}$. 
Now suppose $A^{\bullet}$ is a cdga over $\mathbb{C}$, and $\boldsymbol{X}$ a derived $\mathbb{C}$-scheme with $\boldsymbol{X} \simeq$ $\operatorname{Spec} A^{\bullet}$ in $\mathbf{d S c h}_{\mathbb{C}}$. Then we have an equivalence of triangulated categories $L_{\mathrm{qcoh}}(\boldsymbol{X}) \simeq D(\bmod A)$, which identifies cotangent complexes $\mathbb{L}_{\boldsymbol{X}} \simeq \mathbb{L}_{A} \bullet$. If also $A^{\bullet}$ is of standard form then $\mathbb{L}_{A} \bullet \simeq \Omega_{A}^{1} \bullet$, so $\mathbb{L}_{\boldsymbol{X}} \simeq \Omega_{A}^{1} \bullet$.

Bussi, Brav and Joyce [4, Th. 4.1] prove:

Theorem 2.5. Suppose $\boldsymbol{X}$ is a derived $\mathbb{C}$-scheme (as always, assumed locally finitely presented), and $x \in \boldsymbol{X}$. Then there exists a standard form cdga $A^{\bullet}$ over $\mathbb{C}$ and a Zariski open inclusion $\boldsymbol{\alpha}: \operatorname{Spec} A^{\bullet} \hookrightarrow \boldsymbol{X}$ with $x \in \operatorname{Im} \boldsymbol{\alpha}$.

See Remark 2.2 on the difference in definitions of 'standard form'. Bussi et al. also explain [4, Th. 4.2] how to compare two such standard form charts Spec $A^{\bullet} \hookrightarrow \boldsymbol{X}, \operatorname{Spec} B^{\bullet} \hookrightarrow \boldsymbol{X}$ on their overlap in $\boldsymbol{X}$, using a third chart. We will need the following conditions on derived $\mathbb{C}$-schemes and their morphisms.

Definition 2.6. A derived $\mathbb{C}$-scheme $\boldsymbol{X}$ is called separated, or proper, or quasicompact, if the classical $\mathbb{C}$-scheme $X=t_{0}(\boldsymbol{X})$ is separated, or proper, or quasicompact, respectively, in the classical sense, as in Hartshorne [16. pp. 80, $96,100]$. Proper implies sperated. A morphism of derived schemes $\boldsymbol{f}: \boldsymbol{X} \rightarrow \boldsymbol{Y}$ is proper if $t_{0}(\boldsymbol{f}): t_{0}(\boldsymbol{X}) \rightarrow t_{0}(\boldsymbol{Y})$ is proper in the classical sense [16, p. 100].

We will need the following nontrivial fact about the relation between classical and derived $\mathbb{C}$-schemes. As in Toën [34, $\$ 2.2$, p. 186], a derived $\mathbb{C}$-scheme $\boldsymbol{X}$ is affine if and only if the classical $\mathbb{C}$-scheme $X=t_{0}(\boldsymbol{X})$ is affine.

Recall that a morphism $\alpha: X \rightarrow Y$ in $\mathbf{S c h}_{\mathbb{C}}$ (or $\boldsymbol{\alpha}: \boldsymbol{X} \rightarrow \boldsymbol{Y}$ in $\mathbf{d S c h}_{\mathbb{C}}$ ) is affine if whenever $\beta: U \rightarrow Y$ is a Zariski open inclusion with $U$ affine (or $\boldsymbol{\beta}: \boldsymbol{U} \rightarrow \boldsymbol{Y}$ is Zariski open with $\boldsymbol{U}$ affine), the fibre product $X \times_{\alpha, Y, \beta} U$ in $\mathbf{S c h}_{\mathbb{C}}$ (or homotopy fibre product $\boldsymbol{X} \times{ }_{\boldsymbol{\alpha}, \boldsymbol{Y}, \boldsymbol{\beta}}^{h} \boldsymbol{U}$ in $\mathbf{d S c h}_{\mathbb{C}}$ ) is also affine. Since $\boldsymbol{X}$ is affine if and only if $X=t_{0}(\boldsymbol{X})$ is affine, we see that a morphism $\boldsymbol{\alpha}: \boldsymbol{X} \rightarrow \boldsymbol{Y}$ in $\mathbf{d S c h}_{\mathbb{C}}$ is affine if and only if $t_{0}(\boldsymbol{\alpha}): t_{0}(\boldsymbol{X}) \rightarrow t_{0}(\boldsymbol{Y})$ is affine.

Now let $\boldsymbol{X}$ be a separated derived $\mathbb{C}$-scheme. Then $X=t_{0}(\boldsymbol{X})$ is a separated classical $\mathbb{C}$-scheme, so [16, p. 96] the diagonal morphism $\Delta_{X}: X \rightarrow X \times X$ is a closed immersion. But closed immersions are affine, and $\Delta_{X}=t_{0}\left(\Delta_{\boldsymbol{X}}\right)$ for $\Delta_{\boldsymbol{X}}: \boldsymbol{X} \rightarrow \boldsymbol{X} \times \boldsymbol{X}$ the derived diagonal morphism, so $\Delta_{\boldsymbol{X}}$ is also affine. That is, $\boldsymbol{X}$ has affine diagonal. Therefore if $\boldsymbol{U}_{1}, \boldsymbol{U}_{2} \hookrightarrow \boldsymbol{X}$ are Zariski open inclusions with $\boldsymbol{U}_{1}, \boldsymbol{U}_{2}$ affine, then $\boldsymbol{U}_{1} \times_{\boldsymbol{X}}^{h} \boldsymbol{U}_{2} \hookrightarrow \boldsymbol{X}$ is also Zariski open with $\boldsymbol{U}_{1} \times{ }_{\boldsymbol{X}}^{h} \boldsymbol{U}_{2}$ affine. Thus, finite intersections of open affine derived $\mathbb{C}$-subschemes in a separated derived $\mathbb{C}$-scheme $\boldsymbol{X}$ are affine.

\subsection{PTVV's shifted symplectic geometry}

Next we summarize parts of the theory of shifted symplectic geometry, as developed by Pantev, Toën, Vaquié, and Vezzosi in [30. We explain them for derived $\mathbb{C}$-schemes $\boldsymbol{X}$, although Pantev et al. work more generally with derived stacks.

Given a (locally finitely presented) derived $\mathbb{C}$-scheme $\boldsymbol{X}$ and $p \geqslant 0, k \in \mathbb{Z}$, Pantev et al. 30] define complexes of $k$-shifted p-forms $\mathcal{A}_{\mathbb{C}}^{p}(\boldsymbol{X}, k)$ and $k$-shifted closed $p$-forms $\mathcal{A}_{\mathbb{C}}^{p, \mathrm{cl}}(\boldsymbol{X}, k)$. These are defined first for affine derived $\mathbb{C}$-schemes 
$\boldsymbol{Y}=\operatorname{Spec} A^{\bullet}$ for $A^{\bullet}$ a cdga over $\mathbb{C}$, and shown to satisfy étale descent. Then for general $\boldsymbol{X}, k$-shifted (closed) $p$-forms are defined as a mapping stack; basically, a $k$-shifted (closed) $p$-form $\boldsymbol{\omega}$ on $\boldsymbol{X}$ is the functorial choice for all $\boldsymbol{Y}, \boldsymbol{f}$ of a $k$-shifted (closed) $p$-form $\boldsymbol{f}^{*}(\omega)$ on $\boldsymbol{Y}$ whenever $\boldsymbol{Y}=\operatorname{Spec} A^{\bullet}$ is affine and $\boldsymbol{f}: \boldsymbol{Y} \rightarrow \boldsymbol{X}$ is a morphism.

Definition 2.7. Let $\boldsymbol{Y} \simeq \operatorname{Spec} A^{\bullet}$ be an affine derived $\mathbb{C}$-scheme, for $A^{\bullet}$ a cdga over $\mathbb{C}$. A $k$-shifted $p$-form on $\boldsymbol{Y}$ for $k \in \mathbb{Z}$ is an element $\omega_{A} \bullet \in\left(\Lambda^{p} \mathbb{L}_{A^{\bullet}}\right)^{k}$ with $\mathrm{d} \omega_{A} \bullet=0$ in $\left(\Lambda^{p} \mathbb{L}_{A} \bullet\right)^{k+1}$, so that $\omega_{A} \bullet$ defines a cohomology class $\left[\omega_{A} \bullet\right] \in$ $H^{k}\left(\Lambda^{p} \mathbb{L}_{A} \bullet\right)$. When $p=2$, we call $\omega_{A} \bullet$ nondegenerate, or a $k$-shifted presymplectic form, if the induced morphism $\omega_{A} \bullet: \mathbb{T}_{A} \bullet \rightarrow \mathbb{L}_{A} \bullet[k]$ is a quasi-isomorphism.

A $k$-shifted closed $p$-form on $\boldsymbol{Y}$ is a sequence $\omega_{A}^{*} \bullet=\left(\omega_{A}^{0} \bullet, \omega_{A}^{1} \bullet, \omega_{A}^{2}, \ldots\right)$ such that $\omega_{A \bullet}^{m} \in\left(\Lambda^{p+m} \mathbb{L}_{A} \bullet\right)^{k-m}$ for $m \geqslant 0$, with $\mathrm{d} \omega_{A}^{0} \bullet=0$ and $\mathrm{d} \omega_{A}^{1+m}+\mathrm{d}_{\mathrm{dR}} \omega_{A}^{m}=0$ in $\left(\Lambda^{p+m+1} \mathbb{L}_{A} \bullet\right)^{k-m}$ for all $m \geqslant 0$. Note that if $\omega_{A}^{*} \bullet=\left(\omega_{A}^{0} \bullet, \omega_{A}^{1} \bullet, \ldots\right)$ is a $k$-shifted closed $p$-form then $\omega_{A}^{0}$ e is a $k$-shifted $p$-form.

When $p=2$, we call a $k$-shifted closed 2 -form $\omega_{A}^{*}$ • a $k$-shifted symplectic form if the associated 2 -form $\omega_{A}^{0}$ • is nondegenerate (presymplectic).

If $\boldsymbol{X}$ is a general derived $\mathbb{C}$-scheme, then Pantev et al. [30, §1.2] define $k$ shifted 2-forms $\omega_{\boldsymbol{X}}$, which may be nondegenerate (presymplectic), and $k$-shifted closed 2-forms $\omega_{\boldsymbol{X}}^{*}$, which have an associated $k$-shifted 2 -form $\omega_{\boldsymbol{X}}^{0}$, and where $\omega_{\boldsymbol{X}}^{*}$ is called a $k$-shifted symplectic form if $\omega_{\boldsymbol{X}}^{0}$ is nondegenerate (presymplectic). We will not go into the details of this definition for general $\boldsymbol{X}$.

The important thing for us is this: if $\boldsymbol{Y} \subseteq \boldsymbol{X}$ is a Zariski open affine derived $\mathbb{C}$-subscheme with $\boldsymbol{Y} \simeq \operatorname{Spec} A^{\bullet}$ then a $k$-shifted 2-form $\omega_{\boldsymbol{X}}$ (or a $k$-shifted closed 2-form $\omega_{\boldsymbol{X}}^{*}$ ) on $\boldsymbol{X}$ induces a $k$-shifted 2-form $\omega_{A}$ • (or a $k$-shifted closed 2 -form $\left.\omega_{A}^{*} \bullet\right)$ on $\boldsymbol{Y}$ in the sense above, where $\omega_{A} \bullet$ is unique up to cohomology in the complex $\left(\left(\Lambda^{2} \mathbb{L}_{A} \bullet\right)^{*}, \mathrm{~d}\right)$ (or $\omega_{A}^{*}$ • is unique up to cohomology in the complex $\left.\left(\prod_{m \geqslant 0}\left(\Lambda^{2+m} \mathbb{L}_{A} \bullet\right)^{*-m}, \mathrm{~d}+\mathrm{d}_{\mathrm{dR}}\right)\right)$, and $\omega_{\boldsymbol{X}}$ nondegenerate/presymplectic (or $\omega_{\boldsymbol{X}}^{*}$ symplectic) implies $\omega_{A}$ • nondegenerate/presymplectic (or $\omega_{A}^{*}$ • symplectic).

It is easy to show that if $\boldsymbol{X}$ is a derived $\mathbb{C}$-scheme with a $k$-shifted symplectic or presymplectic form, then $k \leqslant 0$, and the complex virtual dimension vdim $\mathbb{C} \boldsymbol{X}$ satisfies $\operatorname{vdim}_{\mathbb{C}} \boldsymbol{X}=0$ if $k$ is odd, and $\operatorname{vdim}_{\mathbb{C}} \boldsymbol{X}$ is even if $k \equiv 0 \bmod 4$ (which includes classical complex symplectic schemes when $k=0$ ), and $\operatorname{vdim}_{\mathbb{C}} \boldsymbol{X} \in \mathbb{Z}$ if $k \equiv 2 \bmod 4$. In particular, in the case $k=-2$ of interest in this paper, $\operatorname{vdim}_{\mathbb{C}} \boldsymbol{X}$ can take any value in $\mathbb{Z}$.

The main examples we have in mind come from Pantev et al. [30, §2.1]:

Theorem 2.8. Suppose $Y$ is a Calabi-Yau $m$-fold over $\mathbb{C}$, and $\mathcal{M}$ a derived moduli stack of coherent sheaves (or complexes of coherent sheaves) on $Y$. Then $\mathcal{M}$ has a natural $(2-m)$-shifted symplectic form $\omega_{\mathcal{M}}$.

In particular, derived moduli schemes and stacks on a Calabi-Yau 4-fold $Y$ are -2 -shifted symplectic.

Bussi, Brav and Joyce (4) prove 'Darboux Theorems' for $k$-shifted symplectic derived $\mathbb{C}$-schemes $\left(\boldsymbol{X}, \omega_{\boldsymbol{X}}\right)$ for $k<0$, which give explicit Zariski local models for $\left(\boldsymbol{X}, \omega_{\boldsymbol{X}}\right)$. We will explain their main result for $k=-2$. The next definition is taken from [4, Ex. 5.16] (with notation changed, $2 q_{j} s_{j}$ in place of $s_{j}$ ). 
Definition 2.9. A pair $\left(A^{\bullet}, \omega_{A} \bullet\right)$ is called in -2-Darboux form if $A^{\bullet}$ is a standard form cdga over $\mathbb{C}$, and $\omega_{A} \bullet \in\left(\Lambda^{2} \mathbb{L}_{A} \bullet\right)^{-2}=\left(\Lambda^{2} \Omega_{A}^{1} \bullet\right)^{-2}$ with $\mathrm{d} \omega_{A} \bullet=0$ in $\left(\Lambda^{2} \mathbb{L}_{A} \bullet\right)^{-1}$ and $\mathrm{d}_{\mathrm{dR}} \omega_{A} \bullet=0$ in $\left(\Lambda^{3} \mathbb{L}_{A} \bullet\right)^{-2}$, so that $\omega_{A}^{*} \bullet:=\left(\omega_{A} \bullet, 0,0, \ldots\right)$ is a -2-shifted closed 2-form on $A^{\bullet}$, such that:

(i) $A^{0}$ is a smooth $\mathbb{C}$-algebra of dimension $m$, and there exist $x_{1}, \ldots, x_{m}$ in $A^{0}$ forming an étale coordinate system on $V=\operatorname{Spec} A^{0}$.

(ii) The commutative graded algebra $A^{*}$ is freely generated over $A^{0}$ by elements $y_{1}, \ldots, y_{n}$ of degree -1 and $z_{1}, \ldots, z_{m}$ of degree -2 .

(iii) There are invertible elements $q_{1}, \ldots, q_{n}$ in $A^{0}$ such that

$$
\begin{aligned}
\omega_{A} \bullet= & \mathrm{d}_{\mathrm{dR}} z_{1} \mathrm{~d}_{\mathrm{dR}} x_{1}+\cdots+\mathrm{d}_{\mathrm{dR}} z_{m} \mathrm{~d}_{\mathrm{dR}} x_{m} \\
& \quad+\mathrm{d}_{\mathrm{dR}}\left(q_{1} y_{1}\right) \mathrm{d}_{\mathrm{dR}} y_{1}+\cdots+\mathrm{d}_{\mathrm{dR}}\left(q_{n} y_{n}\right) \mathrm{d}_{\mathrm{dR}} y_{n} .
\end{aligned}
$$

(iv) There are elements $s_{1}, \ldots, s_{n} \in A^{0}$ satisfying

$$
q_{1}\left(s_{1}\right)^{2}+\cdots+q_{n}\left(s_{n}\right)^{2}=0 \text { in } A^{0},
$$

such that the differential d on $A^{\bullet}=\left(A^{*}, \mathrm{~d}\right)$ is given by

$$
\mathrm{d} x_{i}=0, \quad \mathrm{~d} y_{j}=s_{j}, \quad \mathrm{~d} z_{i}=\sum_{j=1}^{n} y_{j}\left(2 q_{j} \frac{\partial s_{j}}{\partial x_{i}}+s_{j} \frac{\partial q_{j}}{\partial x_{i}}\right) .
$$

Here the only assumptions are that $A^{0}, x_{1}, \ldots, x_{m}$ are as in (i) and we are given $q_{1}, \ldots, q_{n}, s_{1}, \ldots, s_{n}$ in $A^{0}$ satisfying (2.2), and everything else follows from these. Defining $A^{*}$ as in (ii) and d as in (2.3), then $A^{\bullet}=\left(A^{*}, \mathrm{~d}\right)$ is a standard form cdga over $\mathbb{C}$, where to show that $\mathrm{d} \circ \mathrm{d} z_{i}=0$ we apply $\frac{\partial}{\partial x_{i}}$ to (2.2). Clearly $\mathrm{d}_{\mathrm{dR}} \omega_{A} \bullet=0$, as $\mathrm{d}_{\mathrm{dR}} \circ \mathrm{d}_{\mathrm{dR}}=0$. We have

$$
\begin{aligned}
& \mathrm{d} \omega_{A} \bullet=\sum_{i=1}^{m}\left(\mathrm{~d} \circ \mathrm{d}_{\mathrm{dR}} z_{i}\right) \mathrm{d}_{\mathrm{dR}} x_{i}+\sum_{j=1}^{n}\left(\mathrm{~d} \circ \mathrm{d}_{\mathrm{dR}}\left(q_{j} y_{j}\right)\right) \mathrm{d}_{\mathrm{dR}} y_{j}+\left(\mathrm{d}_{0} \circ \mathrm{d}_{\mathrm{dR}} y_{j}\right) \mathrm{d}_{\mathrm{dR}}\left(q_{j} y_{j}\right) \\
& =-\mathrm{d}_{\mathrm{dR}} \sum_{i=1}^{m} \mathrm{~d} z_{i} \mathrm{~d}_{\mathrm{dR}} x_{i}-\mathrm{d}_{\mathrm{dR}} \sum_{j=1}^{n}\left[\mathrm{~d}\left(q_{j} y_{j}\right) \mathrm{d}_{\mathrm{dR}} y_{j}+\mathrm{d} y_{j} \mathrm{~d}_{\mathrm{dR}}\left(q_{j} y_{j}\right)\right] \\
& =-\mathrm{d}_{\mathrm{dR}} \sum_{i=1}^{m} \sum_{j=1}^{n} y_{j}\left(2 q_{j} \frac{\partial s_{j}}{\partial x_{i}}+s_{j} \frac{\partial q_{j}}{\partial x_{i}}\right) \mathrm{d}_{\mathrm{dR}} x_{i}-\mathrm{d}_{\mathrm{dR}} \sum_{j=1}^{n}\left[q_{j} s_{j} \mathrm{~d}_{\mathrm{dR}} y_{j}+s_{j} \mathrm{~d}_{\mathrm{dR}}\left(q_{j} y_{j}\right)\right] \\
& =-\mathrm{d}_{\mathrm{dR}} \circ \mathrm{d}_{\mathrm{dR}} \sum_{j=1}^{n}\left[\left(q_{j} s_{j}\right) y_{j}+s_{j}\left(q_{j} y_{j}\right)\right]=0,
\end{aligned}
$$

using (2.1) and $\operatorname{dod}_{\mathrm{dR}} x_{i}=0$ for degree reasons in the first step, $\mathrm{d}_{\mathrm{dR}}=-\mathrm{d}_{\mathrm{dR}} \circ \mathrm{d}$ and $\mathrm{d}_{\mathrm{dR}} \circ \mathrm{d}_{\mathrm{dR}}=0$ in the second, (2.3) in the third, $\mathrm{d} s_{j}=\sum_{i=1}^{n} \frac{\partial s_{j}}{\partial x_{i}} \mathrm{~d}_{\mathrm{dR}} x_{i}$ and similarly for $q_{j}$ in the fourth, and $\mathrm{d}_{\mathrm{dR}} \circ \mathrm{d}_{\mathrm{dR}}=0$ in the fifth. Hence $\omega_{A}^{*}$. is a -2-shifted closed 2-form on $A^{\bullet}$. 
The action $\omega_{A} \bullet: \mathbb{T}_{A} \bullet \rightarrow \mathbb{L}_{A} \bullet[-2]$ is given by

$$
\begin{gathered}
\omega_{A} \cdot \frac{\partial}{\partial x_{i}}=-\mathrm{d}_{\mathrm{dR}} z_{i}+\sum_{j=1}^{n} \frac{\partial q_{j}}{\partial x_{i}} y_{j} \mathrm{~d}_{\mathrm{dR}} y_{j} \\
\omega_{A} \cdot \frac{\partial}{\partial y_{j}}=2 q_{j} \mathrm{~d}_{\mathrm{dR}} y_{j}-\sum_{i=1}^{m} y_{j} \frac{\partial q_{j}}{\partial x_{i}} \mathrm{~d}_{\mathrm{dR}} x_{i}, \quad \omega_{A} \cdot \frac{\partial}{\partial z_{i}}=\mathrm{d}_{\mathrm{dR}} x_{i} .
\end{gathered}
$$

By writing this as an upper triangular matrix with invertible diagonal (since the $q_{j}$ are invertible), we see that $\omega_{A} \bullet$ is actually an isomorphism of complexes, so a quasi-isomorphism, and $\omega_{A}^{*}$ is a -2 -shifted symplectic form on $A^{\bullet}$.

The main result of Bussi, Brav and Joyce [4, Th. 5.18] when $k=-2$ yields:

Theorem 2.10. Suppose $\left(\boldsymbol{X}, \omega_{\boldsymbol{X}}^{*}\right)$ is a-2-shifted symplectic derived $\mathbb{C}$-scheme. Then for each $x \in X=t_{0}(\boldsymbol{X})$ there exists a pair $\left(A^{\bullet}, \omega_{A} \bullet\right)$ in -2 -Darboux form and a Zariski open inclusion $\boldsymbol{\alpha}: \operatorname{Spec} A^{\bullet} \hookrightarrow \boldsymbol{X}$ such that $x \in \operatorname{Im} \boldsymbol{\alpha}$ and $\boldsymbol{\alpha}^{*}\left(\omega_{\boldsymbol{X}}^{*}\right) \simeq \omega_{A} \bullet$ in $\mathcal{A}_{\mathbb{C}}^{2, \mathrm{cl}}\left(\mathbf{S p e c} A^{\bullet},-2\right)$. Furthermore, we can choose $A^{\bullet}$ minimal at $x$, in the sense that $m=\operatorname{dim} H^{0}\left(\left.\mathbb{T}_{\boldsymbol{X}}\right|_{x}\right), n=\operatorname{dim} H^{1}\left(\left.\mathbb{T}_{\boldsymbol{X}}\right|_{x}\right)$ in Definition 2.9.

\subsection{Orientations on $k$-shifted symplectic derived schemes}

If $\boldsymbol{X}$ is a derived $\mathbb{C}$-scheme (always assumed locally finitely presented), with classical $\mathbb{C}$-scheme $X=t_{0}(\boldsymbol{X})$, the cotangent complex $\left.\mathbb{L}_{\boldsymbol{X}}\right|_{X}$ restricted to $X$ is a perfect complex, so it has a determinant line bundle $\operatorname{det}\left(\left.\mathbb{L}_{\boldsymbol{X}}\right|_{X}\right)$ on $X$.

The following notion is important for -1-shifted symplectic derived schemes, 3-Calabi-Yau moduli spaces, and generalizations of Donaldson-Thomas theory:

Definition 2.11. Let $\left(\boldsymbol{X}, \omega_{\boldsymbol{X}}^{*}\right)$ be a -1 -shifted symplectic derived $\mathbb{C}$-scheme (or more generally $k$-shifted symplectic, for $k<0$ odd). An orientation for $\left(\boldsymbol{X}, \omega_{\boldsymbol{X}}^{*}\right)$ is a choice of square root line bundle $\operatorname{det}\left(\left.\mathbb{L}_{\boldsymbol{X}}\right|_{X}\right)^{1 / 2}$ for $\operatorname{det}\left(\left.\mathbb{L}_{\boldsymbol{X}}\right|_{X}\right)$.

Writing $X_{\text {an }}$ for the complex analytic topological space of $X$, the obstruction to existence of orientations for $\left(\boldsymbol{X}, \omega_{\boldsymbol{X}}^{*}\right)$ lies in $H^{2}\left(X_{\mathrm{an}} ; \mathbb{Z}_{2}\right)$, and if the obstruction vanishes, the set of orientations is a torsor for $H^{1}\left(X_{\text {an }} ; \mathbb{Z}_{2}\right)$.

This notion of orientation, and its analogue for 'd-critical loci', are used by Ben-Bassat, Brav, Bussi, Dupont, Joyce, Meinhardt, and Szendrői in a series of papers [2 5, 21]. They use orientations on $\left(\boldsymbol{X}, \omega_{\boldsymbol{X}}^{*}\right)$ to define natural perverse sheaves, $\mathcal{D}$-modules, mixed Hodge modules, and motives on $X$. A similar idea first appeared in Kontsevich and Soibelman [25, §5] as 'orientation data' needed to define motivic Donaldson-Thomas invariants of Calabi-Yau 3-folds.

This paper concerns -2-shifted symplectic derived schemes, and 4-CalabiYau moduli spaces. It turns out that there is a parallel notion of orientation in the -2 -shifted case, needed to construct virtual cycles.

To define this, note that determinant line bundles $\operatorname{det}\left(E^{\bullet}\right)$ of perfect complexes $\mathcal{E}^{\bullet}$ satisfy $\operatorname{det}\left[\left(E^{\bullet}\right)^{\vee}\right] \cong\left[\operatorname{det}\left(E^{\bullet}\right)\right]^{-1}$, and $\operatorname{det}\left(E^{\bullet}[k]\right) \cong\left[\operatorname{det}\left(E^{\bullet}\right)\right]^{(-1)^{k}}$. If $\left(\boldsymbol{X}, \omega_{\boldsymbol{X}}^{*}\right)$ is a $k$-shifted symplectic derived $\mathbb{C}$-scheme, then $\mathbb{T}_{\boldsymbol{X}} \simeq \mathbb{L}_{\boldsymbol{X}}[k]$, where 
$\mathbb{T}_{\boldsymbol{X}} \simeq\left(\mathbb{L}_{\boldsymbol{X}}\right)^{\vee}$. Restricting to $X$ and taking determinant line bundles gives $\operatorname{det}\left(\left.\mathbb{L}_{\boldsymbol{X}}\right|_{X}\right)^{-1} \cong \operatorname{det}\left(\left.\mathbb{L}_{\boldsymbol{X}}\right|_{X}\right)^{(-1)^{k}}$. If $k$ is odd this is trivial, but for $k$ even, this gives a canonical isomorphism of line bundles on $X$ :

$$
\iota_{\boldsymbol{X}, \omega_{X}^{*}}:\left[\operatorname{det}\left(\left.\mathbb{L}_{\boldsymbol{X}}\right|_{X}\right)\right]^{\otimes^{2}} \longrightarrow \mathcal{O}_{X} \cong \mathcal{O}_{X}^{\otimes^{2}} .
$$

The next definition is new, so far as the authors know.

Definition 2.12. Let $\left(\boldsymbol{X}, \omega_{\boldsymbol{X}}^{*}\right)$ be a -2 -shifted symplectic derived $\mathbb{C}$-scheme (or more generally $k$-shifted symplectic, for $k<0$ with $k \equiv 2 \bmod 4$ ). An orientation for $\left(\boldsymbol{X}, \omega_{\boldsymbol{X}}^{*}\right)$ is a choice of isomorphism $o: \operatorname{det}\left(\left.\mathbb{L}_{\boldsymbol{X}}\right|_{X}\right) \rightarrow \mathcal{O}_{X}$ such

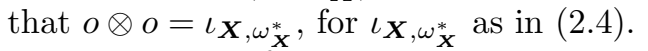

Writing $X_{\text {an }}$ for the complex analytic topological space of $X$, the obstruction to existence of orientations for $\left(\boldsymbol{X}, \omega_{\boldsymbol{X}}^{*}\right)$ lies in $H^{1}\left(X_{\mathrm{an}} ; \mathbb{Z}_{2}\right)$, and if the obstruction vanishes, the set of orientations is a torsor for $H^{0}\left(X_{\mathrm{an}} ; \mathbb{Z}_{2}\right)$.

This definition makes sense for $k$-shifted symplectic derived $\mathbb{C}$-schemes with $k$ even, but when $k \equiv 0 \bmod 4$ (including the classical symplectic case $k=0$ ) there is a natural choice of orientation $o$, so we restrict to $k \equiv 2 \bmod 4$.

At a point $x \in X_{\text {an }}$, we have a canonical isomorphism

$$
\operatorname{det}\left(\left.\mathbb{L}_{\boldsymbol{X}}\right|_{x}\right) \cong \Lambda^{\text {top }} H^{0}\left(\left.\mathbb{L}_{\boldsymbol{X}}\right|_{x}\right) \otimes\left[\Lambda^{\text {top }} H^{-1}\left(\left.\mathbb{L}_{\boldsymbol{X}}\right|_{x}\right)\right]^{*} \otimes \Lambda^{\text {top }} H^{-2}\left(\left.\mathbb{L}_{\boldsymbol{X}}\right|_{x}\right) .
$$

Now $H^{-1}\left(\left.\mathbb{L}_{\boldsymbol{X}}\right|_{x}\right) \cong H^{1}\left(\left.\mathbb{T}_{\boldsymbol{X}}\right|_{x}\right)^{*}$, and $\left.\omega_{\boldsymbol{X}}^{0}\right|_{x}$ gives $H^{0}\left(\left.\mathbb{L}_{\boldsymbol{X}}\right|_{x}\right) \cong H^{-2}\left(\left.\mathbb{L}_{\boldsymbol{X}}\right|_{x}\right)^{*}$, so $\Lambda^{\text {top }} H^{0}\left(\left.\mathbb{L}_{\boldsymbol{X}}\right|_{x}\right) \cong\left[\Lambda^{\text {top }} H^{-2}\left(\left.\mathbb{L}_{\boldsymbol{X}}\right|_{x}\right)\right]^{*}$. Thus we have a canonical isomorphism

$$
\operatorname{det}\left(\left.\mathbb{L}_{\boldsymbol{X}}\right|_{x}\right) \cong \Lambda^{\text {top }} H^{1}\left(\left.\mathbb{T}_{\boldsymbol{X}}\right|_{x}\right) .
$$

Write $Q_{x}$ for the nondegenerate, symmetric $\mathbb{C}$-bilinear pairing

$$
Q_{x}:=\left.\omega_{\boldsymbol{X}}^{0}\right|_{x}: H^{1}\left(\left.\mathbb{T}_{\boldsymbol{X}}\right|_{x}\right) \times H^{1}\left(\left.\mathbb{T}_{\boldsymbol{X}}\right|_{x}\right) \longrightarrow \mathbb{C} .
$$

The determinant $\operatorname{det} Q_{x}$ is an isomorphism $\left[\Lambda^{\text {top }} H^{1}\left(\left.\mathbb{T}_{\boldsymbol{X}}\right|_{x}\right)\right]^{\otimes^{2}} \rightarrow \mathbb{C}$, and $\operatorname{det} Q_{x}$ corresponds to $\left.\iota_{\boldsymbol{X}, \omega_{\boldsymbol{X}}^{*}}\right|_{x}$ under the isomorphism (2.5). There is a natural bijection

$$
\left\{\text { orientations on }\left(\boldsymbol{X}, \omega_{\boldsymbol{X}}^{*}\right) \text { at } x\right\} \cong\left\{\mathbb{C} \text {-orientations on }\left(H^{1}\left(\left.\mathbb{T}_{\boldsymbol{X}}\right|_{x}\right), Q_{x}\right)\right\} \text {. }
$$

To see this, note that if $\left(e_{1}, \ldots, e_{n}\right)$ is an orthonormal basis for $\left(H^{1}\left(\left.\mathbb{T}_{\boldsymbol{X}}\right|_{x}\right), Q_{x}\right)$ then $e_{1} \wedge \cdots \wedge e_{n}$ lies in $\Lambda^{\text {top }} H^{1}\left(\left.\mathbb{T}_{\boldsymbol{X}}\right|_{x}\right)$ with $\operatorname{det} Q_{x}:\left[e_{1} \wedge \cdots \wedge e_{n}\right]^{\otimes^{2}} \mapsto 1$. Orientations for $\left(\boldsymbol{X}, \omega_{\boldsymbol{X}}^{*}\right)$ at $x$ give isomorphisms $\lambda: \Lambda^{\text {top }} H^{1}\left(\left.\mathbb{T}_{\boldsymbol{X}}\right|_{x}\right) \rightarrow \mathbb{C}$ with $\lambda^{2}=\operatorname{det} Q_{x}$, and these correspond to orientations for $\left(H^{1}\left(\left.\mathbb{T}_{\boldsymbol{X}}\right|_{x}\right), Q_{x}\right)$ such that $\lambda: e_{1} \wedge \cdots \wedge e_{n} \mapsto 1$ if $\left(e_{1}, \ldots, e_{n}\right)$ is an oriented orthonormal basis.

\subsection{Kuranishi atlases}

We now define our notion of Kuranishi atlas on a topological space $X$. These are a simplification of m-Kuranishi spaces in [22, §4.7], which in turn are based on the 'Kuranishi spaces' of Fukaya, Oh, Ohta, Ono [14,15]. 
Definition 2.13. Let $X$ be a topological space. A Kuranishi neighbourhood on $X$ is a quadruple $(V, E, s, \psi)$ such that:

(a) $V$ is a smooth manifold.

(b) $\pi: E \rightarrow V$ is a real vector bundle over $V$, called the obstruction bundle.

(c) $s: V \rightarrow E$ is a smooth section of E, called the Kuranishi section.

(d) $\psi$ is a homeomorphism from $s^{-1}(0)$ to an open subset $R=\operatorname{Im} \psi$ in $X$, where $\operatorname{Im} \psi=\left\{\psi(x): x \in s^{-1}(0)\right\}$ is the image of $\psi$.

If $S \subseteq X$ is open, by a Kuranishi neighbourhood over $S$, we mean a Kuranishi neighbourhood $(V, E, s, \psi)$ on $X$ with $S \subseteq \operatorname{Im} \psi \subseteq X$.

Definition 2.14. Let $\left(V_{J}, E_{J}, s_{J}, \psi_{J}\right),\left(V_{K}, E_{K}, s_{K}, \psi_{K}\right)$ be Kuranishi neighbourhoods on a topological space $X$, and $S \subseteq \operatorname{Im} \psi_{J} \cap \operatorname{Im} \psi_{K} \subseteq X$ be open. A coordinate change $\Phi_{J K}:\left(V_{J}, E_{J}, s_{J}, \psi_{J}\right) \rightarrow\left(V_{K}, E_{K}, s_{K}, \psi_{K}\right)$ over $S$ is a triple $\Phi_{J K}=\left(V_{J K}, \phi_{J K}, \hat{\phi}_{J K}\right)$ satisfying:

(a) $V_{J K}$ is an open neighbourhood of $\psi_{J}^{-1}(S)$ in $V_{J}$.

(b) $\phi_{J K}: V_{J K} \rightarrow V_{K}$ is a smooth map.

(c) $\hat{\phi}_{J K}:\left.E_{J}\right|_{V_{J K}} \rightarrow \phi_{J K}^{*}\left(E_{K}\right)$ is a morphism of vector bundles on $V_{J K}$.

(d) $\hat{\phi}_{J K}\left(\left.s_{J}\right|_{V_{J K}}\right)=\phi_{J K}^{*}\left(s_{K}\right)$.

(e) $\psi_{J}=\psi_{K} \circ \phi_{J K}$ on $s_{J}^{-1}(0) \cap V_{J K}$.

(f) Let $x \in S$, and set $v_{J}=\psi_{J}^{-1}(x) \in V_{J}$ and $v_{K}=\psi_{K}^{-1}(x) \in V_{K}$. Then the following is an exact sequence of real vector spaces:

$$
\left.\left.0 \rightarrow T_{v_{J}} V_{J} \stackrel{\left.\left.\mathrm{d} s_{J}\right|_{v_{J}} \oplus \mathrm{d} \phi_{J K}\right|_{v_{J}}}{\longrightarrow} E_{J}\right|_{v_{J}} \oplus T_{v_{K}} V_{K} \stackrel{-\left.\left.\hat{\phi}_{J K}\right|_{v_{J}} \oplus \mathrm{d} s_{K}\right|_{v_{K}}}{\longrightarrow} E_{K}\right|_{v_{K}} \rightarrow 0 .
$$

We can compose coordinate changes: if $\Phi_{J K}=\left(V_{J K}, \phi_{J K}, \hat{\phi}_{J K}\right):\left(V_{J}, E_{J}\right.$, $\left.s_{J}, \psi_{J}\right) \rightarrow\left(V_{K}, E_{K}, s_{K}, \psi_{K}\right)$ and $\Phi_{K L}\left(V_{K L}, \phi_{K L}, \hat{\phi}_{K L}\right):\left(V_{K}, E_{K}, s_{K}, \psi_{K}\right) \rightarrow$ $\left(V_{L}, E_{L}, s_{L}, \psi_{L}\right)$ are coordinate changes over $S_{J K}, S_{K L}$, then

$$
\begin{aligned}
\Phi_{K L} \circ \Phi_{J K}:= & \left(V_{J K} \cap \phi_{J K}^{-1}\left(V_{K L}\right), \phi_{K L} \circ \phi_{J K}\left|\ldots, \phi_{J K}^{*}\left(\hat{\phi}_{K L}\right) \circ \hat{\phi}_{J K}\right| \ldots\right): \\
& \left(V_{J}, E_{J}, s_{J}, \psi_{J}\right) \longrightarrow\left(V_{L}, E_{L}, s_{L}, \psi_{L}\right)
\end{aligned}
$$

is a coordinate change over $S_{J K} \cap S_{K L}$.

Definition 2.15. A Kuranishi atlas $\mathcal{K}$ of virtual dimension $n$ on a topological space $X$ is data $\mathcal{K}=\left(A, \prec,\left(V_{J}, E_{J}, s_{J}, \psi_{J}\right)_{J \in A}, \Phi_{J K}, J \prec K \in A\right)$, where:

(a) $A$ is an indexing set (not necessarily finite).

(b) $\prec$ is a partial order on $A$, where by convention $J \prec K$ only if $J \neq K$.

(c) $\left(V_{J}, E_{J}, s_{J}, \psi_{J}\right)$ is a Kuranishi neighbourhood on $X$ for each $J \in A$, with $\operatorname{dim} V_{J}-\operatorname{rank} E_{J}=n$.

(d) The images $\operatorname{Im} \psi_{J} \subseteq X$ for $J \in A$ have the property that if $J, K \in A$ with $J \neq K$ and $\operatorname{Im} \psi_{J} \cap \operatorname{Im} \psi_{K} \neq \emptyset$ then either $J \prec K$ or $K \prec J$. 
(e) $\Phi_{J K}=\left(V_{J K}, \phi_{J K}, \hat{\phi}_{J K}\right):\left(V_{J}, E_{J}, s_{J}, \psi_{J}\right) \rightarrow\left(V_{K}, E_{K}, s_{K}, \psi_{K}\right)$ is a coordinate change for all $J, K \in A$ with $J \prec K$, over $S=\operatorname{Im} \psi_{J} \cap \operatorname{Im} \psi_{K}$.

(f) $\Phi_{K L} \circ \Phi_{J K}=\Phi_{J L}$ for all $J, K, L \in A$ with $J \prec K \prec L$.

(g) $\bigcup_{J \in A} \operatorname{Im} \psi_{J}=X$.

We call $\mathcal{K}$ a finite Kuranishi atlas if the indexing set $A$ is finite.

If $X$ has a Kuranishi atlas then it is locally compact. In applications we invariably impose extra global topological conditions on $X$, for instance $X$ might be assumed to be compact and Hausdorff; or Hausdorff and second countable; or metrizable; or Hausdorff and paracompact.

We will also need a relative version of Kuranishi atlas in 3.7 Suppose $Z$ is a manifold, and $\pi: X \rightarrow Z$ a continuous map. A relative Kuranishi atlas for $\pi: X \rightarrow Z$ is a Kuranishi atlas $\mathcal{K}$ on $X$ as above, together with smooth maps $\varpi_{J}: V_{J} \rightarrow Z$ for $J \in A$, such that $\left.\varpi_{J}\right|_{s_{J}^{-1}(0)}=\pi \circ \psi_{J}: s_{J}^{-1}(0) \rightarrow Z$ for all $J \in A$, and $\left.\varpi_{J}\right|_{V_{J K}}=\varpi_{K} \circ \phi_{J K}: V_{J K} \rightarrow Z$ for all $J \prec K$ in $A$.

Definition 2.16. Let $X$ be a topological space, with a Kuranishi atlas $\mathcal{K}$ as in Definition 2.15. For each $J \in A$ we can form the $C^{\infty}$ real line bundle $\Lambda^{\text {top }} T^{*} V_{J} \otimes \Lambda^{\text {top }} E_{J}$ over $V_{J}$, where $\Lambda^{\text {top }}(\cdots)$ means the top exterior power. Thus we can form the restriction

$$
\left.\left(\Lambda^{\mathrm{top}} T^{*} V_{J} \otimes \Lambda^{\mathrm{top}} E_{J}\right)\right|_{s_{J}^{-1}(0)} \longrightarrow s_{J}^{-1}(0),
$$

considered as a topological real line bundle over the topological space $s_{J}^{-1}(0)$.

If $J \prec K$ in $A$ then for each $v_{J}$ in $s_{J}^{-1}(0) \cap V_{J K}$ with $\phi_{J K}\left(v_{J}\right)=v_{K}$ in $s_{K}^{-1}(0)$ we have an exact sequence (2.8). Taking top exterior powers in (2.8) (and using a suitable orientation convention) gives an isomorphism

$$
\left.\left.\Lambda^{\mathrm{top}} T_{v_{J}}^{*} V_{J} \otimes \Lambda^{\mathrm{top}} E_{J}\right|_{v_{J}} \cong \Lambda^{\mathrm{top}} T_{v_{K}}^{*} V_{K} \otimes \Lambda^{\mathrm{top}} E_{K}\right|_{v_{K}} .
$$

This depends continuously on $v_{J}, v_{K}$, and so induces an isomorphism of topological line bundles on $s_{J}^{-1}(0) \cap V_{J K}$

$$
\left(\Phi_{J K}\right)_{*}:\left.\left.\left(\Lambda^{\mathrm{top}} T^{*} V_{J} \otimes \Lambda^{\mathrm{top}} E_{J}\right)\right|_{s_{J}^{-1}(0) \cap V_{J K}} \longrightarrow \phi_{J K}\right|_{\ldots} ^{*}\left(\Lambda^{\mathrm{top}} T^{*} V_{K} \otimes \Lambda^{\mathrm{top}} E_{K}\right) .
$$

If $J \prec K \prec L$ in $A$ then as $\Phi_{K L} \circ \Phi_{J K}=\Phi_{J L}$ by Definition 2.15)(f), we see that $\left(\Phi_{K L}\right)_{*} \circ\left(\Phi_{J K}\right)_{*}=\left(\Phi_{J L}\right)_{*}$ in topological line bundles over $s_{J}^{-1}(0) \cap V_{J K} \cap V_{J L}$.

An orientation on $(X, \mathcal{K})$ is a choice of orientation on the fibres of the topological real line bundle $\left.\left(\Lambda^{\mathrm{top}} T^{*} V_{J} \otimes \Lambda^{\mathrm{top}} E_{J}\right)\right|_{s_{J}^{-1}(0)}$ on $s_{J}^{-1}(0)$ for all $J \in A$, such that $\left(\Phi_{J K}\right)_{*}$ is orientation-preserving on $s_{J}^{-1}(0) \cap V_{J K}$ for all $J \prec K$ in $A$.

An equivalent way to think about this is that there is a natural topological real line bundle $K_{X} \rightarrow X$ called the canonical bundle with given isomorphisms

$$
\iota_{J}:\left.\left(\Lambda^{\text {top }} T^{*} V_{J} \otimes \Lambda^{\text {top }} E_{J}\right)\right|_{s_{J}^{-1}(0)} \longrightarrow \psi_{J}^{*}\left(K_{X}\right)
$$

for $J \in A$, such that $\left.\iota_{J}\right|_{s_{J}^{-1}(0) \cap V_{J K}}=\phi_{J K}^{*}\left(\iota_{K}\right) \circ\left(\Phi_{J K}\right)_{*}$ for all $J \prec K$ in $A$, and an orientation on $(X, \mathcal{K})$ is an orientation on the fibres of $K_{X}$. 
Remark 2.17. (a) Our Kuranishi atlases are based on Joyce's 'm-Kuranishi spaces' $22, \S 4.7]$. They are similar to Fukaya-Oh-Ohta-Ono's 'good coordinate systems' [14, Lem. A1.11], [15, Def. 6.1], and McDuff-Wehrheim's 'Kuranishi atlases' 27 28. Our orientations are based on [15, Def. 5.8] and [14, Def. A1.17].

There are two important differences with [14,15,27,28. Firstly, 14,15,27,28] use Kuranishi neighbourhoods $(V, E, \Gamma, s, \psi)$, where $\Gamma$ is a finite group acting equivariantly on $V, E, s$ and $\psi$ maps $s^{-1}(0) / \Gamma \rightarrow X$. This is because their Kuranishi spaces are a kind of derived orbifolds, not derived manifolds.

Secondly, [14, 15, 27, 28, use a more restrictive notion of coordinate change $\Phi_{J K}=\left(V_{J K}, \phi_{J K}, \hat{\phi}_{J K}\right)$, in which $\phi_{J K}: V_{J K} \hookrightarrow V_{K}$ must be an embedding, and $\hat{\phi}_{J K}:\left.E_{J}\right|_{V_{J K}} \hookrightarrow \phi_{J K}^{*}\left(E_{K}\right)$ an embedding of vector bundles, so that $\operatorname{dim} V_{J} \leqslant \operatorname{dim} V_{K}$ and $\operatorname{rank} E_{J} \leqslant \operatorname{rank} E_{K}$. In the Kuranishi atlases we construct later, $\phi_{J K}: V_{J K} \rightarrow V_{K}$ will be a submersion, and $\hat{\phi}_{J K}:\left.E_{J}\right|_{V_{J K}} \rightarrow \phi_{J K}^{*}\left(E_{K}\right)$ will be surjective, so that $\operatorname{dim} V_{J} \geqslant \operatorname{dim} V_{K}$ and $\operatorname{rank} E_{J} \geqslant \operatorname{rank} E_{K}$. That is, our coordinate changes actually go the opposite way to those in [14, 15, 27, 28].

(b) Similar structures to Kuranishi atlases are studied [14, 15,22,27,28, because it is natural to construct them on many differential-geometric moduli spaces. Broadly speaking, any moduli space of solutions of a smooth nonlinear elliptic p.d.e. on a compact manifold should admit a Kuranishi atlas. References [14, 15, 27,28] concern moduli spaces of $J$-holomorphic curves in symplectic geometry.

\subsection{Derived smooth manifolds and virtual classes}

Readers of this paper do not need to know what a derived manifold is. Here is a brief summary of the points relevant to this paper:

- 'Derived manifolds' are derived versions of smooth manifolds, where 'derived' is in the sense of Derived Algebraic Geometry.

- There are several different versions, due to Spivak [31, Borisov-Noel 6, 7 , and Joyce [18 20,22, which form $\infty$-categories or 2-categories. They all include ordinary manifolds Man as a full subcategory.

- All these versions are roughly equivalent. There are natural 1-1 correspondences between equivalence classes of derived manifolds in each theory.

- Much of classical differential geometry generalizes nicely to derived manifolds - submersions, orientations, transverse fibre products, ....

- Given a Hausdorff, second countable topological space $X$ with a Kuranishi atlas $\mathcal{K}$ of dimension $n$, we can construct a derived manifold $\boldsymbol{X}$ with topological space $X$ and dimension $\operatorname{vim} \boldsymbol{X}=n$, unique up to equivalence. Orientations on $(X, \mathcal{K})$ are in 1-1 correspondence with orientations on $\boldsymbol{X}$.

- Compact, oriented derived manifolds $\boldsymbol{X}$ have virtual classes $[\boldsymbol{X}]_{\mathrm{virt}}$ in homology or bordism, generalizing the fundamental class $[X] \in H_{\operatorname{dim} X}(X ; \mathbb{Z})$ of a compact oriented manifold $X$.

- These virtual classes are used to define enumerative invariants such as Gromov-Witten, Donaldson, and Donaldson-Thomas invariants. Such invariants are unchanged under deformations of the underlying geometry. 
- Given a compact Hausdorff topological space $X$ with an oriented Kuranishi atlas $\mathcal{K}$, we could construct the virtual class $[\boldsymbol{X}]_{\text {virt }}$ directly from $(X, \mathcal{K})$, as in [14, 15, 27, 28], without going via the derived manifold $\boldsymbol{X}$.

Readers who do not want to know more details can now skip forward to $\$ 3$

\subsubsection{Different definitions of derived manifold}

The earliest reference to derived differential geometry we are aware of is a short final paragraph by Jacob Lurie [26, §4.5]. Broadly following [26, §4.5], Lurie's student David Spivak 31] constructed an $\infty$-category DerMan $_{\mathbf{S p i}}$ of 'derived manifolds'. Borisov and Noël [7] gave a simplified version, an $\infty$-category $\operatorname{DerMan}_{\mathrm{BoNo}}$, and showed that $\operatorname{DerMan}_{\mathrm{Spi}} \simeq \operatorname{DerMan}_{\mathrm{BoNo}}$.

Joyce 18 20] defined 2-categories dMan of 'd-manifolds' (a kind of derived manifold), and dOrb of 'd-orbifolds' (a kind of derived orbifold), and also strict 2-categories of d-manifolds and d-orbifolds with boundary $\mathbf{d M a n}^{\mathbf{b}}, \mathbf{d O r b}^{\mathbf{b}}$ and with corners $\mathbf{d M a n}^{\mathbf{c}}, \mathbf{d O r b}^{\mathbf{c}}$, and studied their differential geometry in detail.

Borisov 6] constructed a 2-functor $F: \pi_{2}\left(\right.$ DerMan $\left._{\mathrm{BoNo}}\right) \rightarrow$ dMan, where $\pi_{2}\left(\right.$ DerMan $\left._{\mathbf{B o N o}}\right)$ is the 2-category truncation of $\operatorname{DerMan}_{\mathbf{B o N o}}$, and proved that $F$ is close to being an equivalence of 2-categories.

All of [6, 7, 18, 20, 26, 31] use ' $C^{\infty}$-algebraic geometry', as in Joyce [17, a version of (derived) algebraic geometry in which rings are replaced by ' $C^{\infty}$ rings', and define derived manifolds to be special kinds of 'derived $C^{\infty}$-schemes'.

In 22, Joyce gave an alternative approach to derived differential geometry based on the work of Fukaya et al. [14, 15]. He defined 2-categories of ' $\mathrm{m}$ Kuranishi spaces' mKur, a kind of derived manifold, and 'Kuranishi spaces' Kur, a kind of derived orbifold. Here m-Kuranishi spaces are similar to a pair $(X, \mathcal{K})$ of a Hausdorff, second countable topological space $X$ and a Kuranishi atlas $\mathcal{K}$ in the sense of 22.5 In 23 . Joyce defines equivalences of 2-categories $\mathbf{d M a n} \simeq \mathbf{m K u r}$ and $\mathbf{d O r b} \simeq \mathbf{K u r}$, showing that the two approaches to derived differential geometry of [18 20] and [22] are essentially the same.

\subsubsection{Orientations on derived manifolds}

Derived manifolds have a good notion of orientation, which behaves much like orientations on ordinary manifolds. Some references are Joyce [18, §4.8], [19, $\S 4.8],[20, \S 4.6]$ for d-manifolds, Joyce [23] for m-Kuranishi spaces, and Fukaya, Oh, Ohta and Ono [15, §5], 14, §A1.1] for Kuranishi spaces in their sense.

For any kind of derived manifold $\boldsymbol{X}$, we can define a (topological or $C^{\infty}$ ) real line bundle $K_{\boldsymbol{X}}$ over the topological space $X$ called the canonical bundle. It is the determinant line bundle of the cotangent complex $\mathbb{L}_{\boldsymbol{X}}$. For each $x \in \boldsymbol{X}$ we can define a tangent space $T_{x} \boldsymbol{X}$ and obstruction space $O_{x} \boldsymbol{X}$, and then

$$
\left.K_{\boldsymbol{X}}\right|_{x} \cong \Lambda^{\text {top }} T_{x}^{*} \boldsymbol{X} \otimes_{\mathbb{R}} \Lambda^{\text {top }} O_{x} \boldsymbol{X} .
$$

An orientation on $\boldsymbol{X}$ is an orientation on the fibres of $K_{\boldsymbol{X}}$. In a similar way to (2.7), at a single point $x \in X$ we have a natural bijection

$$
\{\text { orientations on } \boldsymbol{X} \text { at } x\} \cong\left\{\text { orientations on } T_{x}^{*} \boldsymbol{X} \oplus O_{x} \boldsymbol{X}\right\} \text {. }
$$


If $(V, E, s, \psi)$ is a Kuranishi neighbourhood on $\boldsymbol{X}$ and $v \in s^{-1}(0) \subseteq V$ with $\psi(v)=x \in \boldsymbol{X}$, then there is a natural exact sequence

$$
\left.0 \longrightarrow T_{x} \boldsymbol{X} \longrightarrow T_{v} V \stackrel{\left.\mathrm{d} s\right|_{v}}{\longrightarrow} E\right|_{v} \longrightarrow O_{x} \boldsymbol{X} \longrightarrow 0 \text {. }
$$

Taking top exterior powers in (2.10) gives an isomorphism

$$
\left.\left.K_{\boldsymbol{X}}\right|_{x} \cong \Lambda^{\mathrm{top}} T_{x}^{*} \boldsymbol{X} \otimes_{\mathbb{R}} \Lambda^{\mathrm{top}} O_{x} \boldsymbol{X} \cong \Lambda^{\mathrm{top}} T_{v}^{*} V \otimes_{\mathbb{R}} \Lambda^{\mathrm{top}} E\right|_{v},
$$

and thus, with a suitable orientation convention, a natural bijection

$$
\{\text { orientations on } \boldsymbol{X} \text { at } x\} \cong\left\{\text { orientations on }\left.T_{v}^{*} V \oplus E\right|_{v}\right\} \text {. }
$$

\subsubsection{Kuranishi atlases and derived manifolds}

The next theorem relates topological spaces with Kuranishi atlases to derived manifolds. The assumption that $X$ is Hausdorff and second countable is just to match the global topological assumptions in [7, 18, 20, 22, 31]. For the last part we restrict to (a),(b) as orientations have not been written down for the theories of (c),(d), although this would not be very difficult.

Theorem 2.18. Let $X$ be a Hausdorff, second countable topological space with a Kuranishi atlas $\mathcal{K}$ of dimension $n$ in the sense of $\$ 2.5$. Then we can construct

(a) An $\boldsymbol{m}$-Kuranishi space $\boldsymbol{X}$ in the sense of Joyce [22, §4.7].

(b) A d-manifold $\boldsymbol{X}$ in the sense of Joyce [18 20 .

(c) A derived manifold in the sense of Borisov and Noël [7].

(d) A derived manifold in the sense of Spivak [31.

In each case $\boldsymbol{X}$ has topological space $X$ and dimension $\operatorname{vdim} \boldsymbol{X}=n$, and $\boldsymbol{X}$ is canonical up to equivalence in the 2 -categories $\mathbf{m K u r , d M a n}$ or $\infty$-categories $\operatorname{DerMan}_{\mathbf{B o N o}}$, DerMan $_{\mathbf{S p i}}$. In cases (a),(b) there is a natural 1-1 correspondence between orientations on $\mathcal{K}$, and orientations on $\boldsymbol{X}$ in 23 and [18,20].

If also $Z$ is a manifold, $\pi: X \rightarrow Z$ is continuous, and $\mathcal{K}, \varpi_{J: J \in A}$ is a relative Kuranishi atlas for $\pi: X \rightarrow Z$, then we can construct a morphism of derived manifolds $\boldsymbol{\pi}: \boldsymbol{X} \rightarrow Z$, canonical up to 2-isomorphism, with continuous map $\pi$.

Proof. Part (a) follows from [22, Th. 4.67] in the m-Kuranishi space case, and part (b) from [18, Th. 4.16], in each case with topological space $X$, and $\operatorname{vdim} \boldsymbol{X}=n$, and $\boldsymbol{X}$ canonical up to equivalence in mKur, dMan. Part (c) then follows from (b) and Borisov [6], and part (d) from (c) and Borisov and Noël [7. The 1-1 correspondences of orientations can be proved by comparing Definition 2.16 with $\$ 2.6 .2$. The last part also follows from [18, Th. 4.16].

\subsubsection{Bordism for derived manifolds}

We now discuss bordism, following [18, §4.10], [19, §15] and [20, §13]. 
Definition 2.19. Let $Y$ be a manifold, and $k \in \mathbb{N}$. Consider pairs $(X, f)$, where $X$ is a compact, oriented manifold with $\operatorname{dim} X=k$, and $f: X \rightarrow Y$ is a smooth map. Define an equivalence relation $\sim$ on such pairs by $(X, f) \sim\left(X^{\prime}, f^{\prime}\right)$ if there exists a compact, oriented $(k+1)$-manifold with boundary $W$, a smooth map $e: W \rightarrow Y$, and a diffeomorphism of oriented manifolds $j:-X \amalg X^{\prime} \rightarrow \partial W$, such that $f \amalg f^{\prime}=e \circ i_{W} \circ j$, where $-X$ is $X$ with the opposite orientation, and $i_{W}: \partial W \hookrightarrow W$ is the inclusion map.

Write $[X, f]$ for the $\sim$-equivalence class (bordism class) of a pair $(X, f)$. Define the bordism group $B_{k}(Y)$ of $Y$ to be the set of all such bordism classes $[X, f]$ with $\operatorname{dim} X=k$. It is an abelian group, with zero $0_{Y}=[\emptyset, \emptyset]$, addition $[X, f]+\left[X^{\prime}, f^{\prime}\right]=\left[X \amalg X^{\prime}, f \amalg f^{\prime}\right]$, and inverses $-[X, f]=[-X, f]$.

Define $\Pi_{\mathrm{bo}}^{\text {hom }}: B_{k}(Y) \rightarrow H_{k}(Y ; \mathbb{Z})$ by $\Pi_{\mathrm{bo}}^{\text {hom }}:[X, f] \mapsto f_{*}([X])$, where $H_{*}(-; \mathbb{Z})$ is singular homology, and $[X] \in H_{k}(X ; \mathbb{Z})$ is the fundamental class.

When $Y$ is the point $*$, the maps $f: X \rightarrow *, e: W \rightarrow *$ are trivial, and we can omit them, and consider $B_{k}(*)$ to be the abelian group of bordism classes $[X]$ of compact, oriented, $k$-dimensional manifolds $X$.

As in Conner [11, §I.5], bordism is a generalized homology theory. Results of Thom, Wall and others in [11, §I.2] compute the bordism groups $B_{k}(*)$. We define d-manifold bordism by replacing manifolds $X$ in $[X, f]$ by d-manifolds $\boldsymbol{X}$ :

Definition 2.20. Let $Y$ be a manifold, and $k \in \mathbb{Z}$. Consider pairs $(\boldsymbol{X}, \boldsymbol{f})$, where $\boldsymbol{X} \in \mathbf{d M a n}$ is a compact, oriented d-manifold with $\operatorname{vdim} \boldsymbol{X}=k$, and $\boldsymbol{f}: \boldsymbol{X} \rightarrow Y$ is a 1-morphism in dMan.

Define an equivalence relation $\sim$ between such pairs by $(\boldsymbol{X}, \boldsymbol{f}) \sim\left(\boldsymbol{X}^{\prime}, \boldsymbol{f}^{\prime}\right)$ if there exists a compact, oriented d-manifold with boundary $\boldsymbol{W}$ with vdim $\boldsymbol{W}=$ $k+1$, a 1-morphism $\boldsymbol{e}: \boldsymbol{W} \rightarrow Y$ in $\mathbf{d M a n}^{\mathbf{b}}$, an equivalence of oriented dmanifolds $\boldsymbol{j}:-\boldsymbol{X} \amalg \boldsymbol{X}^{\prime} \rightarrow \partial \boldsymbol{W}$, and a 2-morphism $\eta: \boldsymbol{f} \amalg \boldsymbol{f}^{\prime} \Rightarrow \boldsymbol{e} \circ \boldsymbol{i}_{\boldsymbol{W}} \circ \boldsymbol{j}$, where $\boldsymbol{i}_{\boldsymbol{W}}: \partial \boldsymbol{W} \rightarrow \boldsymbol{W}$ is the natural 1-morphism.

Write $[\boldsymbol{X}, \boldsymbol{f}]$ for the $\sim$-equivalence class (d-bordism class) of a pair $(\boldsymbol{X}, \boldsymbol{f})$. Define the $d$-bordism group $d B_{k}(Y)$ of $Y$ to be the set of d-bordism classes $[\boldsymbol{X}, \boldsymbol{f}]$ with $\operatorname{vdim} \boldsymbol{X}=k$. As for $B_{k}(Y)$, it is an abelian group, with zero $0_{Y}=[\emptyset, \emptyset]$, addition $[\boldsymbol{X}, \boldsymbol{f}]+\left[\boldsymbol{X}^{\prime}, \boldsymbol{f}^{\prime}\right]=\left[\boldsymbol{X} \amalg \boldsymbol{X}^{\prime}, \boldsymbol{f} \amalg \boldsymbol{f}^{\prime}\right]$, and $-[\boldsymbol{X}, \boldsymbol{f}]=[-\boldsymbol{X}, \boldsymbol{f}]$. Define $\Pi_{\mathrm{bo}}^{\mathrm{dbo}}: B_{k}(Y) \rightarrow d B_{k}(Y)$ for $k \geqslant 0$ by $\Pi_{\mathrm{bo}}^{\mathrm{dbo}}:[X, f] \mapsto[X, f]$.

When $Y$ is a point $*$, we can omit $\boldsymbol{f}: \boldsymbol{X} \rightarrow *$, and consider $d B_{k}(*)$ to be the abelian group of d-bordism classes $[\boldsymbol{X}]$ of compact, oriented d-manifolds $\boldsymbol{X}$.

In [20, $\$ 13.2$ ] we show $B_{*}(Y)$ and $d B_{*}(Y)$ are isomorphic. See [31, Th. 2.6] for an analogous (unoriented) result for Spivak's derived manifolds.

Theorem 2.21. For any manifold $Y$, we have $d B_{k}(Y)=0$ for $k<0$, and $\Pi_{\mathrm{bo}}^{\mathrm{dbo}}: B_{k}(Y) \rightarrow d B_{k}(Y)$ is an isomorphism for $k \geqslant 0$.

The main idea of the proof of Theorem 2.21 is that (compact, oriented) dmanifolds $\boldsymbol{X}$ can be turned into (compact, oriented) manifolds $\tilde{X}$ by a small perturbation. By Theorem 2.21, we may define a projection $\Pi_{\mathrm{dbo}}^{\text {hom }}: d B_{k}(Y) \rightarrow$ $H_{k}(Y ; \mathbb{Z})$ for $k \geqslant 0$ by $\Pi_{\mathrm{dbo}}^{\text {hom }}=\Pi_{\mathrm{bo}}^{\text {hom }} \circ\left(\Pi_{\mathrm{bo}}^{\mathrm{dbo}}\right)^{-1}$. We think of $\Pi_{\mathrm{dbo}}^{\text {hom }}$ as a virtual class map, and call $[\boldsymbol{X}]_{\mathrm{virt}}=\Pi_{\mathrm{dbo}}^{\text {hom }}([\boldsymbol{X}, \boldsymbol{f}])$ the virtual class. Virtual classes 
are used in several areas of geometry to construct enumerative invariants using moduli spaces, for example in [14, §A1], [15, §6] for Fukaya-Oh-Ohta-Ono's Kuranishi spaces, and in Behrend and Fantechi [1] in algebraic geometry.

\subsubsection{Virtual classes for derived manifolds in homology}

If $\boldsymbol{X}$ is a compact, oriented derived manifold of dimension $k \in \mathbb{Z}$ we can also define a virtual class $[\boldsymbol{X}]_{\text {virt }}$ in the homology $H_{k}(X ; \mathbb{Z})$ of the underlying topological space $X$, for a suitable homology theory. By [18, Cor. 4.30] or [19, Cor. 4.31] or [20, Th. 4.29], we can choose an embedding $\boldsymbol{f}: \boldsymbol{X} \hookrightarrow \mathbb{R}^{n}$ for $n \gg 0$. If $Y$ is an open neighbourhood of $f(X)$ in $\mathbb{R}^{n}$ then 2.6 .4 defines $\Pi_{\mathrm{dbo}}^{\text {hom }}([\boldsymbol{X}, \boldsymbol{f}])$ in $H_{k}(Y ; \mathbb{Z})$. We also have a pushforward map $f_{*}: H_{k}(X ; \mathbb{Z}) \rightarrow H_{k}(Y ; \mathbb{Z})$.

If $X$ is a Euclidean Neighbourhood Retract (ENR), we can choose $Y$ so that it retracts onto $f(X)$, and then $f_{*}: H_{k}(X ; \mathbb{Z}) \rightarrow H_{k}(Y ; \mathbb{Z})$ is an isomorphism, so we can define the virtual class $[\boldsymbol{X}]_{\text {virt }}=\left(f_{*}\right)^{-1} \circ \Pi_{\mathrm{dbo}}^{\text {hom }}([\boldsymbol{X}, \boldsymbol{f}])$ in ordinary homology $H_{k}(X ; \mathbb{Z})$. This $[\boldsymbol{X}]_{\text {virt }}$ is independent of the choices of $\boldsymbol{f}, n, Y$.

General derived manifolds may not be ENRs. In this case we use a trick the authors learned from McDuff and Wehrheim [28, §7.5]. Choose a sequence $\mathbb{R}^{n} \supseteq$ $Y_{1} \supseteq Y_{2} \supseteq \cdots$ of open neighbourhoods of $f(X)$ in $\mathbb{R}^{n}$ with $f(X)=\bigcap_{i \geqslant 1} Y_{i}$. Now Steenrod homology $H_{*}^{\mathrm{St}}(-; \mathbb{Z})$ (see Milnor [29]) is a homology theory with the nice properties that $H_{*}^{\mathrm{St}}\left(Y_{i} ; \mathbb{Z}\right) \cong H_{*}\left(Y_{i} ; \mathbb{Z}\right)$ as $Y_{i}$ is a manifold, and as $f(X)=\bigcap_{i \geqslant 1} Y_{i}$ we have an isomorphism with the inverse limit

$$
H_{k}^{\mathrm{St}}(f(X) ; \mathbb{Z}) \cong \lim _{i \geqslant 1} H_{k}^{\mathrm{St}}\left(Y_{i} ; \mathbb{Z}\right) .
$$

Cech homology $\check{H}_{*}(-; \mathbb{Q})$ over $\mathbb{Q}$ (the dual $\mathbb{Q}$-vector spaces to Čech cohomology $\left.\check{H}^{*}(-; \mathbb{Q})\right)$ has the same limiting property. Then writing $\boldsymbol{f}_{i}=\boldsymbol{f}: \boldsymbol{X} \rightarrow Y_{i}$, so that $\Pi_{\mathrm{dbo}}^{\mathrm{hom}}\left(\left[\boldsymbol{X}, \boldsymbol{f}_{i}\right]\right) \in H_{k}\left(Y_{i} ; \mathbb{Z}\right) \cong H_{k}^{\mathrm{St}}\left(Y_{i} ; \mathbb{Z}\right)$, using (2.11) we may form $\varliminf_{i \geqslant 1} \Pi_{\mathrm{dbo}}^{\text {hom }}\left(\left[\boldsymbol{X}, \boldsymbol{f}_{i}\right]\right)$ in $H_{k}^{\mathrm{St}}(f(X) ; \mathbb{Z})$, so that

$$
[\boldsymbol{X}]_{\mathrm{virt}}:=\left(f_{*}\right)^{-1}\left[\lim _{\longleftarrow} \geqslant 1 \Pi_{\mathrm{dbo}}^{\text {hom }}\left(\left[\boldsymbol{X}, \boldsymbol{f}_{i}\right]\right)\right]
$$

is a virtual class in $H_{k}^{\mathrm{St}}(X ; \mathbb{Z})$, or similarly in $\check{H}_{k}(X ; \mathbb{Q})$. Here $[\boldsymbol{X}]_{\text {virt }}$ is independent of the choices of $\boldsymbol{f}, n, Y_{i}$.

For the examples in this paper, $X$ is the complex analytic topological space of a proper $\mathbb{C}$-scheme, and therefore an ENR. Then $H_{k}^{\mathrm{St}}(X ; \mathbb{Z}) \cong H_{k}(X ; \mathbb{Z})$ and $\check{H}_{k}(X ; \mathbb{Q}) \cong H_{k}(X ; \mathbb{Q})$, and the virtual class lives in ordinary homology.

\section{The main results}

We now give our main results. We begin in 33.1 with a general existence result for a special kind of atlas for $\boldsymbol{\pi}: \boldsymbol{X} \rightarrow Z$, where $\boldsymbol{X}$ is a separated derived $\mathbb{C}$ scheme and $Z$ a smooth affine classical $\mathbb{C}$-scheme, an atlas in which the charts are spectra of standard form cdgas, the coordinate changes are quasi-free, and composition of coordinate changes is strictly associative.

Sections 3.23 .5 build up to our primary goal, Theorems 3.15 and 3.16 in 93.5. which show that to a separated, -2 -shifted symplectic derived $\mathbb{C}$-scheme 
$\left(\boldsymbol{X}, \omega_{\boldsymbol{X}}^{*}\right)$ with $\operatorname{vdim}_{\mathbb{C}} \boldsymbol{X}=n$ and complex analytic topological space $X_{\text {an }}$, we can build a Kuranishi atlas $\mathcal{K}$ on $X_{\text {an }}$, and so construct a derived manifold $\boldsymbol{X}_{\mathrm{dm}}$ with topological space $X_{\mathrm{an}}$, with $\operatorname{vdim}_{\mathbb{R}} \boldsymbol{X}_{\mathrm{dm}}=n$. In 33.6 we show that orientations on $\left(\boldsymbol{X}, \omega_{\boldsymbol{X}}^{*}\right)$ and on $\left(X_{\mathrm{an}}, \mathcal{K}\right)$ and on $\boldsymbol{X}_{\mathrm{dm}}$ correspond, and prove that for $\left(\boldsymbol{X}, \omega_{\boldsymbol{X}}^{*}\right)$ proper and oriented, the bordism class $\left[\boldsymbol{X}_{\mathrm{dm}}\right] \in d B_{n}(*)$ is a 'virtual cycle' independent of choices.

Section 3.7 extends 33.2 3.6 to families $\left(\boldsymbol{\pi}: \boldsymbol{X} \rightarrow Z,\left[\omega_{\boldsymbol{X} / Z}\right]\right)$ over a connected base $\mathbb{C}$-scheme $Z$, and shows that the bordism class $\left[\boldsymbol{X}_{\mathrm{dm}}^{z}\right] \in d B_{n}(*)$ associated to a fibre $\boldsymbol{\pi}^{-1}(z)$ is independent of $z \in Z_{\text {an }}$. Finally, $\$ 3.8-93.9$ discuss applying our results to define Donaldson-Thomas style invariants 'counting' coherent sheaves on Calabi-Yau 4-folds, and motivation from gauge theory.

\subsection{Zariski homotopy atlases on derived schemes}

Derived schemes and stacks, discussed in 2.2 are very abstract objects, and difficult to do computations with. But standard form cdgas $A^{\bullet}, B^{\bullet}$ and quasifree morphisms $\Phi: A^{\bullet} \rightarrow B^{\bullet}$ in $\$ 2.1$ are easy to work with explicitly. Our first main result, proved in $\$ 4$, constructs well-behaved homotopy atlases for a derived scheme $\boldsymbol{X}$, built from standard form cdgas and quasi-free morphisms.

Theorem 3.1. Let $\boldsymbol{X}$ be a separated derived $\mathbb{C}$-scheme, $Z=\operatorname{Spec} B$ be a smooth classical affine $\mathbb{C}$-scheme for $B$ a smooth $\mathbb{C}$-algebra of pure dimension, and $\boldsymbol{\pi}: \boldsymbol{X} \rightarrow Z$ be a morphism. Suppose we are given data $\left\{\left(A_{i}^{\bullet}, \boldsymbol{\alpha}_{i}, \beta_{i}\right): i \in\right.$ $I\}$, where $I$ is an indexing set and for each $i \in I, A_{i}^{\bullet} \in \mathbf{c d g a}_{\mathbb{C}}$ is a standard form cdga, and $\boldsymbol{\alpha}_{i}: \operatorname{Spec} A_{i}^{\bullet} \hookrightarrow \boldsymbol{X}$ is a Zariski open inclusion in $\mathbf{d S c h}_{\mathbb{C}}$, and $\beta_{i}: B \rightarrow A_{i}^{0}$ is a smooth morphism of classical $\mathbb{C}$-algebras such that the following diagram homotopy commutes in $\mathbf{d S c h}_{\mathbb{C}}$ :

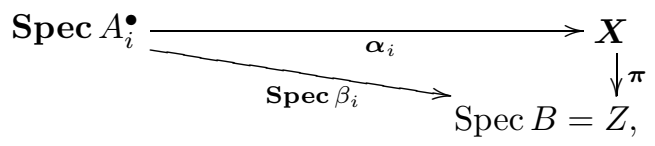

regarding $\beta_{i}$ as a morphism $B \rightarrow A_{i}^{\bullet}$. Then we can construct the following data:

(i) For all finite subsets $\emptyset \neq J \subseteq I$, a standard form cdga $A_{J}^{\bullet} \in$ cdga $_{\mathbb{C}}$, a Zariski open inclusion $\boldsymbol{\alpha}_{J}: \operatorname{Spec} A_{J}^{\bullet} \hookrightarrow \boldsymbol{X}$, with image $\operatorname{Im} \boldsymbol{\alpha}_{J}=$ $\bigcap_{i \in J} \operatorname{Im} \boldsymbol{\alpha}_{i}$, and a smooth morphism of classical $\mathbb{C}$-algebras $\beta_{J}: B \rightarrow A_{J}^{0}$, such that the following diagram homotopy commutes in $\mathbf{d S c h}_{\mathbb{C}}$ :

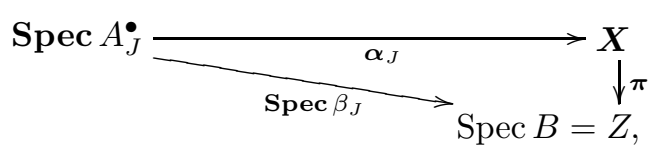

and when $J=\{i\}$ for $i \in I$ we have $A_{\{i\}}^{\bullet}=A_{i}^{\bullet}, \boldsymbol{\alpha}_{\{i\}}=\boldsymbol{\alpha}_{i}$, and $\beta_{\{i\}}=\beta_{i}$.

(ii) For all inclusions of finite subsets $\emptyset \neq K \subseteq J \subseteq I$, a quasi-free morphism of standard form cdgas $\Phi_{J K}: A_{K}^{\bullet} \rightarrow A_{J}^{\bullet}$ with $\beta_{J}=\Phi_{J K} \circ \beta_{K}: B \rightarrow A_{J}^{0}$, 
such that the following diagram homotopy commutes in $\mathbf{d S c h}_{\mathbb{C}}$ :

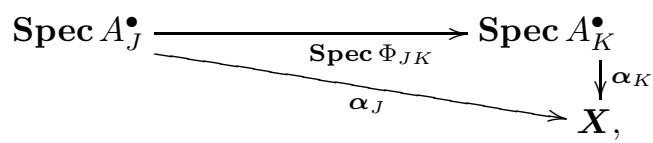

and if $\emptyset \neq L \subseteq K \subseteq J \subseteq I$ then $\Phi_{J L}=\Phi_{J K} \circ \Phi_{K L}: A_{L}^{\bullet} \rightarrow A_{J}^{\bullet}$.

\subsection{Interpreting Zariski atlases using complex geometry}

Given a -2-shifted symplectic derived $\mathbb{C}$-scheme $\left(\boldsymbol{X}, \omega_{\boldsymbol{X}}^{*}\right)$ satisfying conditions, we will construct a derived manifold structure $\boldsymbol{X}_{\mathrm{dm}}$ on the complex analytic topological space $X_{\text {an }}$ underlying $\boldsymbol{X}$. To do this, we need a change of language: we have to pass from talking about derived schemes $\boldsymbol{X}$, cdgas $A^{\bullet}$, etc., to talking about smooth manifolds $V$, vector bundles $E \rightarrow V$, smooth sections $s: V \rightarrow E$, as $\boldsymbol{X}_{\mathrm{dm}}$ will be built by gluing together such local Kuranishi models $(V, E, s)$.

Therefore we now rewrite part of the output $A_{J}^{\bullet}, \beta_{J}: B \rightarrow A_{J}^{0}, \Phi_{J K}$ : $A_{J}^{\bullet} \rightarrow A_{K}^{\bullet}$ of Theorem 3.1 in terms of complex manifolds $V$, holomorphic vector bundles $E \rightarrow V$, and holomorphic sections $s: V \rightarrow E$. In $\$ 3.5$ we will pass to certain real vector bundles $E^{+}=E / E^{-}$to define $\boldsymbol{X}_{\mathrm{dm}}$.

First we interpret standard form cdgas $A^{\bullet} \in \mathbf{c d g a}_{\mathbb{C}}$ using holomorphic data. We discuss only data from degrees $0,-1,-2$ in $A^{\bullet}$, as this is all we need, but one could also define vector bundles $G, H, \ldots$ over $V$ corresponding to $M^{-3}, M^{-4}, \ldots$, and many vector bundle morphisms, satisfying equations.

Definition 3.2. Let $A^{\bullet}=\left(\cdots \rightarrow A^{-2} \stackrel{\mathrm{d}}{\longrightarrow} A^{-1} \stackrel{\mathrm{d}}{\longrightarrow} A^{0}\right)$ be a standard form cdga over $\mathbb{C}$, as in 2.1 Then $A^{0}$ is a finitely generated smooth $\mathbb{C}$-algebra, so $V^{\text {alg }}:=\operatorname{Spec} A^{0}$ is a smooth affine $\mathbb{C}$-scheme, assumed of pure dimension, as in 2.1. Now any $\mathbb{C}$-scheme $S$ has an underlying complex analytic space $S_{\text {an }}$, which is a complex manifold if $S$ is smooth and of pure dimension.

Write $V$ for the complex manifold $\left(V^{\text {alg }}\right)_{\text {an }}$ associated to $V^{\text {alg }}=\operatorname{Spec} A^{0}$.

As $A^{\bullet}$ is of standard form, the graded $\mathbb{C}$-algebra $A^{*}$ is freely generated over $A^{0}$ by a series of finitely generated free $A^{0}$-modules $M^{-1} \subseteq A^{-1}, M^{-2} \subseteq$ $A^{-2}, \ldots$ Thus $A^{-1} \cong M^{-1}, A^{-2} \cong M^{-2} \oplus \Lambda_{A^{0}}^{2} M^{-1}$, and so on, giving

$$
M^{-1}=A^{-1}, \quad M^{-2} \cong A^{-2} / \Lambda_{A^{0}}^{2} A^{-1}, \ldots
$$

Hence, the $M^{i}$ are determined by $A^{*}$ as $A^{0}$-modules up to canonical isomorphism, although for $i \leqslant-2$ the inclusions $M^{i} \hookrightarrow A^{i}$ involve an arbitrary choice.

Now finitely generated free $A^{0}$-modules $M$ are those of the form $M \cong$ $H^{0}\left(C^{\text {alg }}\right)$ for $C^{\text {alg }} \rightarrow V^{\text {alg }}=$ Spec $A^{0}$ a trivial algebraic vector bundle. Write $E^{\text {alg }} \rightarrow V^{\text {alg }}, F^{\text {alg }} \rightarrow V^{\text {alg }}$ for the trivial algebraic vector bundles (unique up to canonical isomorphism) with $M^{-1} \cong H^{0}\left(\left(E^{\text {alg }}\right)^{*}\right), M^{-2} \cong H^{0}\left(\left(F^{\text {alg }}\right)^{*}\right)$. That

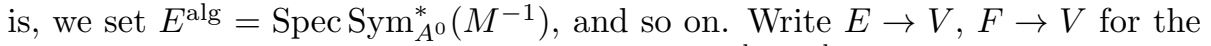
holomorphic vector bundles corresponding to $E^{\mathrm{alg}}, F^{\mathrm{alg}}$. 
We now have isomorphisms

$$
\begin{aligned}
A^{0} & \cong H^{0}\left(\mathcal{O}_{V^{\mathrm{alg} g}}\right), \quad A^{-1} \cong H^{0}\left(\left(E^{\mathrm{alg}}\right)^{*}\right), \\
A^{-2} & \cong H^{0}\left(\left(F^{\mathrm{alg}}\right)^{*}\right) \oplus H^{0}\left(\Lambda^{2}\left(E^{\mathrm{alg}}\right)^{*}\right) .
\end{aligned}
$$

Thus d : $A^{-1} \rightarrow A^{0}$ is identified with an $A^{0}$-module morphism $H^{0}\left(\left(E^{\text {alg }}\right)^{*}\right) \rightarrow$ $H^{0}\left(\mathcal{O}_{V^{\text {alg }}}\right)$, that is, a morphism $\left(E^{\text {alg }}\right)^{*} \rightarrow \mathcal{O}_{V^{\text {alg }}}$ of algebraic vector bundles, which is dual to a morphism $\mathcal{O}_{V^{\text {alg }}} \cong \mathcal{O}_{V^{\text {alg }}}^{*} \rightarrow E^{\text {alg }}$, i.e. a section $s^{\text {alg }} \in$ $H^{0}\left(E^{\text {alg }}\right)$ of $E^{\text {alg }}$. Write $s \in H^{0}(E)$ for the corresponding holomorphic section.

Similarly, write $t^{\text {alg }}: E^{\text {alg }} \rightarrow F^{\text {alg }}$ for the algebraic vector bundle morphism dual to the component of d : $A^{-2} \rightarrow A^{-1}$ mapping $H^{0}\left(\left(F^{\text {alg }}\right)^{*}\right) \rightarrow H^{0}\left(\left(E^{\text {alg }}\right)^{*}\right)$ under (3.5), and write $t: E \rightarrow F$ for the corresponding morphism of holomorphic vector bundles. Then $\mathrm{d} \circ \mathrm{d}=0$ implies that $t \circ s=0: \mathcal{O}_{V} \rightarrow F$.

We should also consider how this data $E, F, s, t$ depends on the choice of inclusion $M^{-2} \hookrightarrow A^{-2}$. Here $E, F$ are independent of choices up to canonical isomorphism, and $s$ is independent of choices. Changing the inclusion $M^{-2} \hookrightarrow A^{-2}$ is equivalent to choosing an algebraic vector bundle morphism $\gamma^{\text {alg }}: \Lambda^{2} E^{\text {alg }} \rightarrow F^{\text {alg }}$ and identifying $M^{-2}$ with the image of id $\oplus\left(\gamma^{\text {alg }}\right)^{*}$ : $H^{0}\left(\left(F^{\text {alg }}\right)^{*}\right) \hookrightarrow H^{0}\left(\left(F^{\text {alg }}\right)^{*}\right) \oplus H^{0}\left(\Lambda^{2}\left(E^{\text {alg }}\right)^{*}\right)$. Writing $\gamma: \Lambda^{2} E \rightarrow F$ for the corresponding holomorphic morphism, this changes $t$ to $\tilde{t}$, where

$$
\tilde{t}=t+\gamma \circ(-\wedge s)
$$

Notice that $\left.t\right|_{v}:\left.\left.E\right|_{v} \rightarrow F\right|_{v}$ is independent of choices at $v \in V$ with $s(v)=0$.

Next suppose $\boldsymbol{X}$ is a derived $\mathbb{C}$-scheme and $\boldsymbol{\alpha}$ : $\mathbf{S p e c} A^{\bullet} \hookrightarrow \boldsymbol{X}$ a Zariski open inclusion. Write $X=t_{0}(\boldsymbol{X})$ for the classical $\mathbb{C}$-scheme, and $X_{\text {an }}$ for the set of $\mathbb{C}$-points of $X$ equipped with the complex analytic topology. (One can give $X_{\text {an }}$ the structure of a complex analytic space, but we will not use this.) Then $t_{0}\left(\mathbf{S p e c} A^{\bullet}\right)$ is the $\mathbb{C}$-subscheme $\left(s^{\text {alg }}\right)^{-1}(0) \subseteq V^{\text {alg }}$, so $\alpha=t_{0}(\boldsymbol{\alpha})$ is a Zariski open inclusion $\left(s^{\text {alg }}\right)^{-1}(0) \hookrightarrow X$. Write $\psi: s^{-1}(0) \hookrightarrow X_{\text {an }}$ for the corresponding map of $\mathbb{C}$-points. Then $\psi$ is a homeomorphism with an open set $R=\operatorname{Im} \psi \subseteq X_{\text {an }}$. Note that $(V, E, s, \psi)$ is a Kuranishi neighbourhood on $X_{\text {an }}$, in the sense of $₫ 2.5$.

As we explained in 2.1 2.2 if $A^{\bullet}$ is a standard form cdga then it is easy to compute the cotangent complex $\mathbb{L}_{A} \bullet \simeq \Omega_{A}^{1} \bullet$, and this also can be identified with the cotangent complex $\mathbb{L}_{\mathbf{S p e c} A} \bullet$ of the derived scheme $\operatorname{Spec} A^{\bullet}$. Let $v \in$ $s^{-1}(0) \subseteq V$ with $\psi(v)=x \in X_{\text {an }}$. Then $v$ is a $\mathbb{C}$-point of Spec $A^{\bullet}$ and $x$ a $\mathbb{C}$ point of $\boldsymbol{X}$ with $\boldsymbol{\alpha}(v)=x$, so $\left.\mathbb{L}_{\boldsymbol{\alpha}}\right|_{v}:\left.\left.\mathbb{L}_{\boldsymbol{X}}\right|_{x} \rightarrow \mathbb{L}_{\mathbf{S p e c} A} \bullet\right|_{v}$ is a quasi-isomorphism, and induces an isomorphism on cohomology. One can show that $\left.\mathbb{L}_{\mathbf{S p e c} A} \cdot\right|_{v}$ is represented by the complex of $\mathbb{C}$-vector spaces

$$
\left.\left.\cdots \longrightarrow F\right|_{v} ^{*} \stackrel{\left.t\right|_{v} ^{*}}{\longrightarrow} E\right|_{v} ^{*} \stackrel{\left.\mathrm{d} s\right|_{v} ^{*}}{\longrightarrow} T_{v}^{*} V \longrightarrow 0,
$$

with $T_{v}^{*} V$ in degree 0 . Dualizing to tangent complexes and taking cohomology, 
we get canonical isomorphisms

$$
\begin{aligned}
& H^{0}\left(\left.\mathbb{T}_{\boldsymbol{\alpha}}\right|_{v}\right): \operatorname{Ker}\left(\left.\mathrm{d} s\right|_{v}:\left.T_{v} V \rightarrow E\right|_{v}\right) \longrightarrow H^{0}\left(\left.\mathbb{T}_{\boldsymbol{X}}\right|_{x}\right), \\
& H^{1}\left(\left.\mathbb{T}_{\boldsymbol{\alpha}}\right|_{v}\right): \frac{\operatorname{Ker}\left(\left.t\right|_{v}:\left.\left.E\right|_{v} \rightarrow F\right|_{v}\right)}{\operatorname{Im}\left(\left.\mathrm{d} s\right|_{v}:\left.T_{v} V \rightarrow E\right|_{v}\right)} \longrightarrow H^{1}\left(\left.\mathbb{T}_{\boldsymbol{X}}\right|_{x}\right) .
\end{aligned}
$$

Now suppose that $Z=\operatorname{Spec} B$ is a smooth classical affine $\mathbb{C}$-scheme of pure dimension, $\boldsymbol{\pi}: \boldsymbol{X} \rightarrow Z$ is a morphism, and $\beta: B \rightarrow A^{0}$ is a smooth morphism of $\mathbb{C}$-algebras, such that as for (3.1) 3.2 the following homotopy commutes

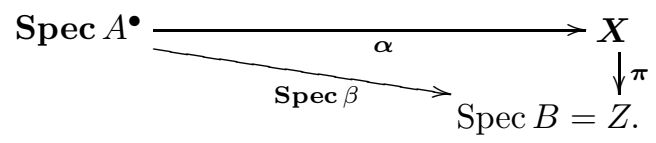

Then $Z_{\text {an }}$ is a complex manifold, and $\tau^{\text {alg }}:=\operatorname{Spec} \beta: V^{\text {alg }} \rightarrow Z$ is a smooth morphism of $\mathbb{C}$-schemes, and $\tau:=\left(\tau^{\text {alg }}\right)_{\text {an }}: V \rightarrow Z_{\text {an }}$ is a holomorphic submersion of complex manifolds. We can form the relative cotangent complexes $\mathbb{L}_{\boldsymbol{X} / Z}, \mathbb{L}_{\text {Spec } A \bullet / Z}$ and dual relative tangent complexes $\mathbb{T}_{\boldsymbol{X} / Z}, \mathbb{T}_{\mathbf{S p e c} A} \bullet / Z$, and (3.10) gives morphisms $\mathbb{L}_{\boldsymbol{\alpha}}: \mathbb{L}_{\boldsymbol{X} / Z} \rightarrow \mathbb{L}_{\text {Spec } A} \bullet / Z, \mathbb{T}_{\boldsymbol{\alpha}}: \mathbb{T}_{\mathbf{S p e c} A} \bullet / Z \rightarrow \mathbb{T}_{\boldsymbol{X} / Z}$.

Write $T\left(V / Z_{\text {an }}\right)=\operatorname{Ker}\left(\mathrm{d} \tau: T V \rightarrow \tau^{*}\left(T Z_{\text {an }}\right)\right)$ for the relative tangent bundle of $V / Z_{\text {an }}$. It is a holomorphic vector subbundle of $T V$ of $\operatorname{rank} \operatorname{dim} V-\operatorname{dim} Z$, as $\tau$ is a holomorphic submersion. Let $v \in s^{-1}(0) \subseteq V$ with $\psi(v)=x \in X_{\text {an }}$ and $\tau(v)=\pi(x)=z \in Z_{\text {an }}$. Then as in (3.7), $\left.\mathbb{L}_{\text {Spec } A \bullet / Z}\right|_{v}$ is represented by the complex of $\mathbb{C}$-vector spaces

$$
\left.\left.\cdots \longrightarrow F\right|_{v} ^{*} \stackrel{\left.t\right|_{v} ^{*}}{\longrightarrow} E\right|_{v} ^{*} \stackrel{\left.\mathrm{d} s\right|_{v} ^{*}}{\longrightarrow} T_{v}^{*}\left(V / Z_{\mathrm{an}}\right) \longrightarrow 0,
$$

with $T_{v}^{*}\left(V / Z_{\text {an }}\right)$ in degree 0 . As for (3.8)-(3.9) we get canonical isomorphisms

$$
\begin{aligned}
& H^{0}\left(\left.\mathbb{T}_{\boldsymbol{\alpha}}\right|_{v}\right): \operatorname{Ker}\left(\left.\mathrm{d} s\right|_{v}:\left.T_{v}\left(V / Z_{\mathrm{an}}\right) \rightarrow E\right|_{v}\right) \longrightarrow H^{0}\left(\left.\mathbb{T}_{\boldsymbol{X} / Z}\right|_{x}\right), \\
& H^{1}\left(\left.\mathbb{T}_{\boldsymbol{\alpha}}\right|_{v}\right): \frac{\operatorname{Ker}\left(\left.t\right|_{v}:\left.\left.E\right|_{v} \rightarrow F\right|_{v}\right)}{\operatorname{Im}\left(\left.\mathrm{d} s\right|_{v}:\left.T_{v}\left(V / Z_{\mathrm{an}}\right) \rightarrow E\right|_{v}\right)} \longrightarrow H^{1}\left(\left.\mathbb{T}_{\boldsymbol{X} / Z}\right|_{x}\right) .
\end{aligned}
$$

Example 3.3. Suppose $\left(A^{\bullet}, \omega_{A} \bullet\right)$ is in -2-Darboux form, in the sense of Definition 2.9, with coordinates $x_{1}, \ldots, x_{m}, y_{1}, \ldots, y_{n}, z_{1}, \ldots, z_{m}$, and 2 -form $\omega_{A}$ • in (2.1), depending on invertible functions $q_{1}, \ldots, q_{n} \in A^{0}$.

Let $V, E, F, s, t$ be as in Definition [3.2. Then $V$ is a smooth $\mathbb{C}$-scheme of dimension $m$, with étale coordinates $\left(x_{1}, \ldots, x_{m}\right)$, so that $T V$ is a trivial vector bundle with basis of sections $\frac{\partial}{\partial x_{1}}, \ldots, \frac{\partial}{\partial x_{m}}$. Also $E$ is a trivial vector bundle of rank $n$, with basis $e_{1}:=\frac{\partial}{\partial y_{1}}, \ldots, e_{n}:=\frac{\partial}{\partial y_{n}}$, and $F$ is trivial of rank $m$, with basis $\frac{\partial}{\partial z_{1}}, \ldots, \frac{\partial}{\partial z_{m}}$. Using the first line of $\omega_{A}$ ・ in (2.1), it is natural to identify $F \cong T^{*} V$ by identifying $\frac{\partial}{\partial z_{i}} \cong \mathrm{d}_{\mathrm{dR}} x_{i}$ for $i=1, \ldots, m$.

The natural section $s \in H^{0}(E)$ is $s=s_{1} e_{1}+\cdots+s_{n} e_{n}$. Write $\epsilon^{1}, \ldots, \epsilon^{n}$ for the basis of sections of $E^{*}$ dual to $e_{1}, \ldots, e_{n}$, so that $\epsilon^{j} \cong \mathrm{d}_{\mathrm{dR}} y_{j}$. Motivated 
by the second line of $\omega_{A}$ • in (2.1), define $Q=q_{1} \epsilon^{1} \otimes \epsilon^{1}+\cdots+q_{n} \epsilon^{n} \otimes \epsilon^{n}$ in $H^{0}\left(S^{2} E^{*}\right)$. Then $Q$ is a natural nondegenerate quadratic form on the fibres of $E$, and (2.2) implies that $Q(s, s)=0$.

Identifying $F=T^{*} V$, from (2.3) we see that $t: E \rightarrow F$ is given by

$$
t\left(e_{j}\right)=\sum_{i=1}^{m}\left(2 q_{j} \frac{\partial s_{j}}{\partial x_{i}}+s_{j} \frac{\partial q_{j}}{\partial x_{i}}\right) \mathrm{d}_{\mathrm{dR}} x_{i}=2 q_{j} \mathrm{~d}_{\mathrm{dR}} s_{j}+s_{j} \mathrm{~d}_{\mathrm{dR}} q_{j},
$$

for $j=1, \ldots, n$. Then $t \circ s=0$ follows from applying $\mathrm{d}_{\mathrm{dR}}$ to $Q(s, s)=0$.

What will matter later is that we have a complex manifold $V$, a holomorphic vector bundle $E \rightarrow V$, a section $s \in H^{0}(E)$, and a nondegenerate holomorphic quadratic form $Q \in H^{0}\left(S^{2} E^{*}\right)$ with $Q(s, s)=0$, such that the classical complex analytic topological space $\left(\operatorname{Spec} H^{0}\left(A^{\bullet}\right)\right)$ an is $s^{-1}(0) \subseteq V$.

Next we interpret quasi-free morphisms of standard form cdgas $\Phi_{J K}: A_{K}^{\bullet} \rightarrow$ $A_{J}^{\bullet}$, as in Theorem 3.1 (a)(ii), in terms of complex geometry.

Definition 3.4. Let $\Phi_{J K}: A_{K}^{\bullet} \rightarrow A_{J}^{\bullet}$ be a quasi-free morphism of standard form cdgas over $\mathbb{C}$, as in $\$ 2.1$. Let $V_{J}^{\mathrm{alg}}, E_{J}^{\mathrm{alg}}, F_{J}^{\mathrm{alg}}, s_{J}^{\mathrm{alg}}, t_{J}^{\mathrm{alg}}, V_{J}, E_{J}, F_{J}, s_{J}, t_{J}$ be as in Definition 3.2 for $A_{J}^{\bullet}$, and $V_{K}^{\text {alg }}, E_{K}^{\text {alg }}, \ldots, t_{K}$ for $A_{K}^{\bullet}$.

Then $\phi_{J K}^{\text {alg }}:=\operatorname{Spec} \Phi_{J K}^{0}: V_{J}^{\text {alg }}=\operatorname{Spec} A_{J}^{0} \rightarrow V_{K}^{\text {alg }}=\operatorname{Spec} A_{K}^{0}$ is a $\mathbb{C}$-scheme morphism. Write $\phi_{J K}: V_{J} \rightarrow V_{K}$ for the corresponding holomorphic map. The quasi-free condition on $\Phi_{J K}$ implies that $\mathrm{d} \phi_{J K}^{\mathrm{alg}}:\left(\phi_{J K}^{\mathrm{alg}}\right)^{*}\left(T^{*} V_{K}^{\mathrm{alg}}\right) \rightarrow T^{*} V_{J}^{\mathrm{alg}}$ is injective, so d $\phi_{J K}: \phi_{J K}^{*}\left(T^{*} V_{K}\right) \rightarrow T^{*} V_{J}$ is injective, that is, $\phi_{J K}: V_{J} \rightarrow V_{K}$ is a submersion of complex manifolds.

Now $\Phi_{J K}^{-1}: A_{K}^{-1} \rightarrow A_{J}^{-1}$ induces an $A_{J}^{0}$-linear map $\left(\Phi_{J K}^{-1}\right)_{*}: A_{K}^{-1} \otimes_{A_{K}^{0}}$ $A_{J}^{0} \rightarrow A_{J}^{-1}$, which under (3.5) corresponds to an algebraic vector bundle mor$\operatorname{phism}\left(\phi_{J K}^{\mathrm{alg}}\right)^{*}\left(\left(E_{K}^{\mathrm{alg}}\right)^{*}\right) \rightarrow\left(E_{J}^{\mathrm{alg}}\right)^{*}$. Write $\chi_{J K}^{\mathrm{alg}}: E_{J}^{\mathrm{alg}} \rightarrow\left(\phi_{J K}^{\mathrm{alg}}\right)^{*}\left(E_{K}^{\mathrm{alg}}\right)$ for the dual morphism, and $\chi_{J K}: E_{J} \rightarrow \phi_{J K}^{*}\left(E_{K}\right)$ for the corresponding morphism of holomorphic vector bundles. It is surjective, as $\Phi_{J K}$ is quasi-free. Then $\mathrm{d} \circ \Phi_{J K}^{-1}=\Phi_{J K}^{0} \circ \mathrm{d}$ implies that

$$
\chi_{J K}\left(s_{J}\right)=\phi_{J K}^{*}\left(s_{K}\right) \in H^{0}\left(\phi_{J K}^{*}\left(E_{K}\right)\right) \text {. }
$$

By (3.4) we have a natural composition of morphisms

$$
H^{0}\left(\left(F_{K}^{\mathrm{alg}}\right)^{*}\right) \cong M_{K}^{-2} \cong A_{K}^{-2} / \Lambda_{A_{K}^{0}}^{2} A_{K}^{-1} \stackrel{\left(\Phi_{J K}^{-2}\right)^{*}}{\longrightarrow} A_{J}^{-2} / \Lambda_{A_{J}^{0}}^{2} A_{J}^{-1} \cong M_{J}^{-2} \cong H^{0}\left(\left(F_{J}^{\mathrm{alg}}\right)^{*}\right) \text {. }
$$

The induced $A_{J}^{0}$-linear map corresponds to a natural algebraic vector bundle morphism $\left(\phi_{J K}^{\mathrm{alg}}\right)^{*}\left(\left(F_{K}^{\mathrm{alg}}\right)^{*}\right) \rightarrow\left(F_{J}^{\mathrm{alg}}\right)^{*}$. Write $\xi_{J K}^{\mathrm{alg}}: F_{J}^{\mathrm{alg}} \rightarrow\left(\phi_{J K}^{\mathrm{alg}}\right)^{*}\left(F_{K}^{\mathrm{alg}}\right)$ for the dual morphism, and $\xi_{J K}: F_{J} \rightarrow \phi_{J K}^{*}\left(F_{K}\right)$ for the corresponding morphism of holomorphic vector bundles. It is surjective, as $\Phi_{J K}$ is quasi-free.

These $\xi_{J K}^{\mathrm{alg}}, \xi_{J K}$ are independent of choices, as they depend on the canonical isomorphism $M^{-2} \cong A^{-2} / \Lambda_{A^{0}}^{2} A^{-1}$ rather than on the non-canonical inclusion $M^{-2} \hookrightarrow A^{-2}$ in Definition [3.2. However, $\Phi_{J K}^{-2}$ need not map $M_{K}^{-2} \subseteq$ $A_{K}^{-2}$ to $M_{J}^{-2} \subseteq A_{J}^{-2}$, and so under the isomorphisms (3.5) need not map $H^{0}\left(\left(F_{K}^{\mathrm{alg}}\right)^{*}\right) \rightarrow H^{0}\left(\left(F_{J}^{\mathrm{alg}}\right)^{*}\right)$. Write $\delta_{J K}^{\mathrm{alg}}: \Lambda^{2} E_{J}^{\mathrm{alg}} \rightarrow\left(\phi_{J K}^{\mathrm{alg}}\right)^{*}\left(F_{K}^{\mathrm{alg}}\right)$ for the algebraic vector bundle morphism dual to the component of $\Phi_{J K}^{-2}$ mapping 
$H^{0}\left(\left(F_{K}^{\mathrm{alg}}\right)^{*}\right) \rightarrow H^{0}\left(\Lambda^{2}\left(E_{J}^{\mathrm{alg}}\right)^{*}\right)$, and $\delta_{J K}: \Lambda^{2} E_{J} \rightarrow \phi_{J K}^{*}\left(F_{K}\right)$ for the corresponding morphism of vector bundles. Then $\mathrm{d} \circ \Phi_{J K}^{-2}=\Phi_{J K}^{-1} \circ \mathrm{d}$ implies that

$$
\xi_{J K} \circ t_{J}+\delta_{J K} \circ\left(-\wedge s_{J}\right)=\phi_{J K}^{*}\left(t_{K}\right) \circ \chi_{J K}: E_{J} \longrightarrow \phi_{J K}^{*}\left(F_{K}\right) .
$$

Thus $\chi_{J K}, \xi_{J K}$ do not strictly commute with $t_{J}, t_{K}$, which is not surprising, since $t_{J}, t_{K}$ depend on arbitrary choices as in (3.6). But notice that $\left.\left.\xi_{J K}\right|_{v} \circ t_{J}\right|_{v}=$ $\left.\left.t_{K}\right|_{\phi_{J K}(v)} \circ \chi_{J K}\right|_{v}$ at $v \in V_{J}$ with $s_{J}(v)=0$.

Next suppose we are given Zariski open inclusions $\boldsymbol{\alpha}_{J}: \operatorname{Spec} A_{J}^{\bullet} \hookrightarrow \boldsymbol{X}$, $\boldsymbol{\alpha}_{K}:$ Spec $A_{K}^{\bullet} \hookrightarrow \boldsymbol{X}$ into a derived $\mathbb{C}$-scheme $\boldsymbol{X}$, such that (3.3) homotopy commutes, and let $\psi_{J}: s_{J}^{-1}(0) \hookrightarrow X_{\text {an }}, \psi_{K}: s_{K}^{-1}(0) \hookrightarrow X_{\text {an }}$ be as in Definition 3.2. As the classical truncation of (3.3) commutes, we see that

$$
\psi_{J}=\left.\psi_{K} \circ \phi_{J K}\right|_{s_{J}^{-1}(0)}: s_{J}^{-1}(0) \longrightarrow X_{\mathrm{an}} .
$$

Suppose $v_{J} \in s_{J}^{-1}(0) \subseteq V_{J}$ with $\phi_{J K}\left(v_{J}\right)=v_{K} \in s_{K}^{-1}(0) \subseteq V_{K}$ and $\psi_{J}\left(v_{J}\right)=\psi_{K}\left(v_{K}\right)=x \in X_{\mathrm{an}}$. As (3.3) homotopy commutes, the corresponding morphisms of tangent complexes $\mathbb{T}_{\mathbf{S p e c} A_{J}^{\bullet}}, \mathbb{T}_{\mathbf{S p e c} A_{K}^{\bullet}}, \mathbb{T}_{\boldsymbol{X}}$ commute up to homotopy, so restricting to $v_{J}, v_{K}, x$ and taking homology gives strictly commuting diagrams. Thus using (3.8)-(3.9), we see that the following diagrams commute:

$$
\begin{aligned}
& \begin{array}{l}
\operatorname{Ker}\left(\left.\mathrm{d} s_{J}\right|_{v_{J}}:\left.T_{v_{J}} V_{J} \rightarrow E_{J}\right|_{v_{J}}\right) \underset{H^{0}\left(\left.\mathbb{T}_{\boldsymbol{\alpha}_{J}}\right|_{v_{J}}\right)}{ } \\
\downarrow^{\left.\left(\left.\mathrm{d} \phi_{J K}\right|_{v_{J}}\right)\right|_{\operatorname{Ker}(\cdots)}} \\
\operatorname{Ker}\left(\left.\mathrm{d} s_{K}\right|_{v_{K}}:\left.T_{v_{K}} V_{K} \rightarrow E_{K}\right|_{v_{K}}\right) \stackrel{H^{0}\left(\left.\mathbb{T}_{\boldsymbol{\alpha}_{K}}\right|_{v_{K}}\right)}{\longrightarrow}\left(\left.\mathbb{T}_{\boldsymbol{X}}\right|_{x}\right),
\end{array} \\
& \begin{array}{l}
\frac{\operatorname{Ker}\left(\left.t_{J}\right|_{v_{J}}:\left.\left.E_{J}\right|_{v_{J}} \rightarrow F_{J}\right|_{v_{J}}\right)}{\operatorname{Im}\left(\left.\mathrm{d} s_{J}\right|_{v_{J}}:\left.T_{v_{J}} V_{J} \rightarrow E_{J}\right|_{v_{K}}\right)} \stackrel{H^{1}\left(\left.\mathbb{T}_{\alpha_{J}}\right|_{v_{J}}\right)}{ } \\
\quad \frac{\operatorname{Ker}\left(\left.\chi_{J K}\right|_{v_{J}}\right)_{*}}{\operatorname{Km}\left(\left.t_{K}\right|_{v_{K}}:\left.\left.E_{K}\right|_{v_{K}} \rightarrow F_{K}\right|_{v_{K}}\right)} \longrightarrow H^{1}\left(\left.\mathbb{T}_{\boldsymbol{X}}\right|_{x}\right) .
\end{array}
\end{aligned}
$$

Now suppose that $Z=\operatorname{Spec} B$ is a smooth classical affine $\mathbb{C}$-scheme of pure dimension, $\boldsymbol{\pi}: \boldsymbol{X} \rightarrow Z$ is a morphism, and $\beta_{J}: B \rightarrow A_{J}^{0}, \beta_{K}: B \rightarrow A_{K}^{0}$ are smooth morphisms of $\mathbb{C}$-algebras, such that (3.2) homotopy commutes for $J, K$, and $\beta_{J}=\Phi_{J K} \circ \beta_{K}$. As in Definition 3.2 we have holomorphic submersions $\tau_{J}: V_{J} \rightarrow Z_{\text {an }}, \tau_{K}: V_{K} \rightarrow Z_{\text {an }}$, with $\tau_{J}=\tau_{K} \circ \phi_{J K}: V_{J} \rightarrow Z_{\text {an }}$ as $\beta_{J}=$ $\Phi_{J K} \circ \beta_{K}$. Let $v_{J} \in s_{J}^{-1}(0) \subseteq V_{J}$ with $\phi_{J K}\left(v_{J}\right)=v_{K} \in s_{K}^{-1}(0) \subseteq V_{K}$, and $\psi_{J}\left(v_{J}\right)=\psi_{K}\left(v_{K}\right)=x \in X_{\mathrm{an}}$, and $\tau_{J}\left(v_{J}\right)=\tau_{K}\left(v_{K}\right)=\pi(x)=z \in Z_{\mathrm{an}}$. Then 
using (3.11)-(3.12), we see that the following diagrams commute:

$$
\begin{aligned}
& \begin{array}{l}
\left.\operatorname{Ker}\left(\left.\mathrm{d} s_{J}\right|_{v_{J}}:\left.T_{v_{J}}\left(V_{J} / Z_{\mathrm{an}}\right) \rightarrow E_{J}\right|_{v_{J}}\right) \underset{H^{0}\left(\mathbb{T}_{\left.\left.\boldsymbol{\alpha}_{J}\right|_{v_{J}}\right)}\right.}{ }\left(\left.\mathrm{d}_{\phi_{K}}\right|_{v_{J}}\right)\right|_{\operatorname{Ker}(\cdots)} \\
\left.\left.\operatorname{Ker}\left(\left.\mathrm{d} s_{K}\right|_{v_{K}}:\left.T_{v_{K}}\left(V_{K} / Z_{\mathrm{an}}\right) \rightarrow E_{K}\right|_{v_{K}}\right) \stackrel{H^{0}\left(\mathbb{T}_{\left.\boldsymbol{\alpha}_{K} \mid v_{K}\right)}^{\longrightarrow} H^{0}\right.}{\longrightarrow} \mathbb{T}_{\boldsymbol{X} / Z}\right|_{x}\right),
\end{array}
\end{aligned}
$$

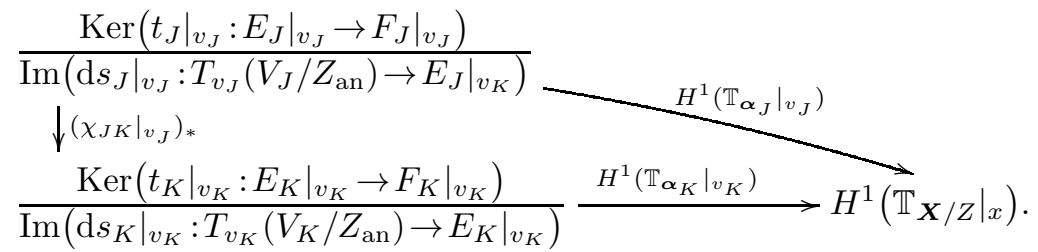

Applying Definitions 3.2 and 3.4 to the conclusions of Theorem 3.1 yields:

Corollary 3.5. In the situation of Theorem 3.1, write $X_{\text {an }}$ for the set of $\mathbb{C}$ points of $X=t_{0}(\boldsymbol{X})$, regarded as a topological space with the complex analytic topology. Then we obtain the following data in complex geometry:

(i) For all finite subsets $\emptyset \neq J \subseteq I$, a complex manifold $V_{J}$, a holomorphic submersion $\tau_{J}: V_{J} \rightarrow Z_{\text {an }}$, holomorphic vector bundles $E_{J}, F_{J} \rightarrow V_{J}, a$ holomorphic section $s_{J}: V_{J} \rightarrow E_{J}$, and a homeomorphism $\psi_{J}: s_{J}^{-1}(0) \rightarrow$ $R_{J} \subseteq X_{\text {an }}$, where $R_{J} \subseteq X_{\text {an }}$ is open, with $\pi \circ \psi_{J}=\left.\tau_{J}\right|_{s_{J}^{-1}(0)}: s_{J}^{-1}(0) \rightarrow$ $Z_{\text {an }}$. These image subsets satisfy $R_{J}=\bigcap_{i \in J} R_{\{i\}}$.

By making an additional arbitrary choice we also obtain a morphism of holomorphic vector bundles $t_{J}: E_{J} \rightarrow F_{J}$, with $t_{J} \circ s_{J}=0$. Different choices $t_{J}, \tilde{t}_{J}$ are related by (3.6). The restrictions $\left.t_{J}\right|_{v_{J}}:\left.\left.E_{J}\right|_{v_{J}} \rightarrow F_{J}\right|_{v_{J}}$ for $v_{J} \in s_{J}^{-1}(0)$ are independent of choices. For each $v_{J} \in s_{J}^{-1}(0)$ with $\psi_{J}\left(v_{J}\right)=x \in X_{\mathrm{an}}$, there are canonical isomorphisms (3.8)-(3.9) writing $H^{i}\left(\left.\mathbb{T}_{\boldsymbol{X}}\right|_{x}\right)$ for $i=0,1$ and (3.11) -(3.12) writing $H^{i}\left(\left.\mathbb{T}_{\boldsymbol{X} / Z}\right|_{x}\right)$ for $i=0,1$ in terms of $V_{J}, E_{J}, F_{J}, s_{J}, t_{J}, \tau_{J}$ at $v_{J}$.

(ii) For all inclusions of finite subsets $\emptyset \neq K \subseteq J \subseteq I$, a holomorphic submersion $\phi_{J K}: V_{J} \rightarrow V_{K}$, and surjective morphisms of holomorphic vector bundles $\chi_{J K}: E_{J} \rightarrow \phi_{J K}^{*}\left(E_{K}\right)$ and $\xi_{J K}: F_{J} \rightarrow \phi_{J K}^{*}\left(F_{K}\right)$. These satisfy $\tau_{J}=\tau_{K} \circ \phi_{J K}: V_{J} \rightarrow Z_{\mathrm{an}}$, and $\chi_{J K}\left(s_{J}\right)=\phi_{J K}^{*}\left(s_{K}\right)$, and $\psi_{J}=$ $\left.\psi_{K} \circ \phi_{J K}\right|_{s_{J}^{-1}(0)}: s_{J}^{-1}(0) \rightarrow X_{\mathrm{an}}$.

If $t_{J}, t_{K}$ are possible choices in (i) then $\chi_{J K}, \xi_{J K}, t_{J}, t_{K}$ are related as in (3.15). If $v_{J} \in s_{J}^{-1}(0)$ with $\phi_{J K}\left(v_{J}\right)=v_{K} \in s_{K}^{-1}(0)$, this implies that

$$
\left.\left.\xi_{J K}\right|_{v_{J}} \circ t_{J}\right|_{v_{J}}=\left.\left.t_{K}\right|_{v_{K}} \circ \chi_{J K}\right|_{v_{J}}:\left.\left.E_{J}\right|_{v_{J}} \longrightarrow F_{K}\right|_{v_{K}} .
$$

If $v_{J} \in s_{J}^{-1}(0) \subseteq V_{J}$ with $\phi_{J K}\left(v_{J}\right)=v_{K} \in s_{K}^{-1}(0) \subseteq V_{K}$ and $\psi_{J}\left(v_{J}\right)=$ $\psi_{K}\left(v_{K}\right)=x \in X_{\text {an }}$, then (3.17)-(3.20) commute.

If $\emptyset \neq L \subseteq K \subseteq J \subseteq I$ then $\phi_{J L}=\phi_{K L} \circ \phi_{J K}, \chi_{J L}=\phi_{J K}^{*}\left(\chi_{K L}\right) \circ \chi_{J K}$, and $\xi_{J L}=\phi_{J K}^{*}\left(\xi_{K L}\right) \circ \xi_{J K}$. 


\subsection{Subbundles $E^{-} \subseteq E$ and Kuranishi neighbourhoods}

Throughout 3.3 , when we apply Theorem 3.1 we take $B=\mathbb{C}$, so that $Z$ is the point $*=\operatorname{Spec} \mathbb{C}$, and the data $\pi, \beta_{i}, \beta_{J}, \tau_{J}$ is trivial, so we omit it.

Suppose $\left(\boldsymbol{X}, \omega_{\boldsymbol{X}}^{*}\right)$ is a -2 -shifted symplectic derived $\mathbb{C}$-scheme, $A^{\bullet}$ a standard form cdga over $\mathbb{C}$, and $\boldsymbol{\alpha}: \operatorname{Spec} A^{\bullet} \rightarrow \boldsymbol{X}$ a Zariski open inclusion. Then Definition 3.2 defines complex geometric data $V, E, F, s, t, \psi, R$, such that $(V, E, s, \psi)$ is a Kuranishi neighbourhood on the topological space $X_{\text {an }}$ of $\boldsymbol{X}$.

However these are not the Kuranishi neighbourhoods we want: they depend only on $\boldsymbol{X}$, not on $\omega_{\boldsymbol{X}}^{*}$, and in general two such neighbourhoods $\left(V_{J}, E_{J}, s_{J}, \psi_{J}\right)$ and $\left(V_{K}, E_{K}, s_{K}, \psi_{K}\right)$ are not compatible over their intersection $R_{J} \cap R_{K}$ in $X_{\text {an }}$ (e.g. the virtual dimensions $\operatorname{dim}_{\mathbb{R}} V_{J}-\operatorname{rank}_{\mathbb{R}} E_{J}$ and $\operatorname{dim}_{\mathbb{R}} V_{K}-\operatorname{rank}_{\mathbb{R}} E_{K}$ may be different), so we cannot glue them to make $X_{\text {an }}$ into a derived manifold.

The basic problem is that the rank of $E$ may be too large - for instance, we can modify $A^{\bullet}$ to replace $E, F, s, t$ by $\tilde{E}=E \oplus G, \tilde{F}=F \oplus G, \tilde{s}=s \oplus 0$, $\tilde{t}=t \oplus \mathrm{id}_{G}$ for some holomorphic vector bundle $G \rightarrow V$. Our solution is to choose a real vector subbundle $E^{-} \subseteq E$ satisfying some conditions involving $\omega_{\boldsymbol{X}}^{*}$, and set $E^{+}=E / E^{-}$to be the quotient bundle and $s^{+}=s+E^{-}$in $C^{\infty}\left(E^{+}\right)$to be the quotient section. The conditions on $E^{-}$imply that $s^{-1}(0)=\left(s^{+}\right)^{-1}(0)$, so $\left(V, E^{+}, s^{+}, \psi^{+}\right)$is also a Kuranishi neighbourhood on $X_{\text {an }}$. Under good conditions we can make two such $\left(V_{J}, E_{J}^{+}, s_{J}^{+}, \psi_{J}^{+}\right),\left(V_{K}, E_{K}^{+}, s_{K}^{+}, \psi_{K}^{+}\right)$compatible over $R_{J} \cap R_{K}$, and glue these local models to make $X_{\text {an }}$ into a derived manifold.

We define the class of subbundles $E^{-} \subseteq E$ we are interested in:

Definition 3.6. Let $\left(\boldsymbol{X}, \omega_{\boldsymbol{X}}^{*}\right)$ be a -2 -shifted symplectic derived $\mathbb{C}$-scheme with $\operatorname{vdim}_{\mathbb{C}} \boldsymbol{X}=n$, and suppose $A^{\bullet} \in \mathbf{c d g a}_{\mathbb{C}}$ is of standard form and $\boldsymbol{\alpha}: A^{\bullet} \hookrightarrow \boldsymbol{X}$ is a Zariski open inclusion. Define complex geometric data $V, E, F, s, t$ and $\psi: s^{-1}(0) \stackrel{\cong}{\longrightarrow} R \subseteq X_{\text {an }}$ as in Definition 3.2 , and suppose $R \neq \emptyset$. Then for each $v \in s^{-1}(0)$ with $\psi(v)=x \in X_{\text {an }}$, equation (3.9) gives an isomorphism from a vector space depending on $V, E, F, s, t$ at $v$ to $H^{1}\left(\left.\mathbb{T}_{\boldsymbol{X}}\right|_{x}\right)$.

Equation (2.6) defined a quadratic form $Q_{x}$ on $H^{1}\left(\left.\mathbb{T}_{\boldsymbol{X}}\right|_{x}\right)$. Define

$$
\tilde{Q}_{v}: \frac{\operatorname{Ker}\left(\left.t\right|_{v}:\left.\left.E\right|_{v} \rightarrow F\right|_{v}\right)}{\operatorname{Im}\left(\left.\mathrm{d} s\right|_{v}:\left.T_{v} V \rightarrow E\right|_{v}\right)} \times \frac{\operatorname{Ker}\left(\left.t\right|_{v}:\left.\left.E\right|_{v} \rightarrow F\right|_{v}\right)}{\operatorname{Im}\left(\left.\mathrm{d} s\right|_{v}:\left.T_{v} V \rightarrow E\right|_{v}\right)} \rightarrow \mathbb{C}
$$

to be the nondegenerate complex quadratic form identified with $Q_{x}$ in (2.6) by the isomorphism $H^{1}\left(\left.\mathbb{T}_{\boldsymbol{\alpha}}\right|_{v}\right)$ in (3.9).

Consider pairs $\left(U, E^{-}\right)$, where $U \subseteq V$ is open and $E^{-}$is a real vector subbundle of $\left.E\right|_{U}$. Given such $\left(U, E^{-}\right)$, we write $E^{+}=\left.E\right|_{U} / E^{-}$for the quotient vector bundle over $U$, and $s^{+} \in C^{\infty}\left(E^{+}\right)$for the image of $\left.s\right|_{U}$ under the projection $\left.E\right|_{U} \rightarrow E^{+}$, and $\psi^{+}:=\left.\psi\right|_{s^{-1}(0) \cap U}: s^{-1}(0) \cap U \rightarrow X_{\mathrm{an}}$. We say that $\left(U, E^{-}\right)$satisfies condition $(*)$ if:

$(*)$ For each $v \in s^{-1}(0) \cap U$, we have

$$
\begin{aligned}
\left.\operatorname{Im}\left(\left.\mathrm{d} s\right|_{v}:\left.T_{v} V \rightarrow E\right|_{v}\right) \cap E^{-}\right|_{v} & =\{0\} & & \text { in }\left.E\right|_{v}, \\
\left.t\right|_{v}\left(\left.E^{-}\right|_{v}\right) & =\left.t\right|_{v}\left(\left.E\right|_{v}\right) & & \text { in }\left.F\right|_{v},
\end{aligned}
$$


and the natural real linear map

$$
\Pi_{v}:\left.E^{-}\right|_{v} \cap \operatorname{Ker}\left(\left.t\right|_{v}:\left.\left.E\right|_{v} \rightarrow F\right|_{v}\right) \longrightarrow \frac{\operatorname{Ker}\left(\left.t\right|_{v}:\left.\left.E\right|_{v} \rightarrow F\right|_{v}\right)}{\operatorname{Im}\left(\left.\mathrm{d} s\right|_{v}:\left.T_{v} V \rightarrow E\right|_{v}\right)},
$$

which is injective by (3.22), has image $\operatorname{Im} \Pi_{v}$ a real vector subspace of dimension exactly half the real dimension of $\operatorname{Ker}\left(\left.t\right|_{v}\right) / \operatorname{Im}\left(\left.\mathrm{d} s\right|_{v}\right)$, and the real quadratic form $\operatorname{Re} \tilde{Q}_{v}$ on $\operatorname{Ker}\left(\left.t\right|_{v}\right) / \operatorname{Im}\left(\left.\mathrm{d} s\right|_{v}\right)$ from (3.21) restricts to a negative definite real quadratic form on $\operatorname{Im} \Pi_{v}$.

We say $\left(U, E^{-}\right)$satisfies condition $(\dagger)$ if:

$(\dagger)\left(U, E^{-}\right)$satisfies condition $(*)$ and $s^{-1}(0) \cap U=\left(s^{+}\right)^{-1}(0) \subseteq U$.

Then $\left(U, E^{+}, s^{+}, \psi^{+}\right)$is a Kuranishi neighbourhood on $X_{\text {an }}$.

Observe that if $v \in s^{-1}(0) \cap U$ with $\psi(v)=x \in X_{\text {an }}$ then using (3.8)-(3.9) and (3.22) - (3.24) we find there is an exact sequence

$$
\left.0 \longrightarrow H^{0}\left(\left.\mathbb{T}_{\boldsymbol{X}}\right|_{x}\right) \longrightarrow T_{v} U \longrightarrow E^{+}\right|_{v} \longrightarrow H^{1}\left(\left.\mathbb{T}_{\boldsymbol{X}}\right|_{x}\right) / \operatorname{Im} \Pi_{v} \longrightarrow 0 .
$$

Hence

$$
\begin{aligned}
& \operatorname{dim}_{\mathbb{R}} U-\operatorname{rank}_{\mathbb{R}} E^{+}=\operatorname{dim}_{\mathbb{R}} H^{0}\left(\left.\mathbb{T}_{\boldsymbol{X}}\right|_{x}\right)-\operatorname{dim}_{\mathbb{R}} H^{1}\left(\left.\mathbb{T}_{\boldsymbol{X}}\right|_{x}\right)+\operatorname{dim}_{\mathbb{R}} \operatorname{Im} \Pi_{v} \\
& =2 \operatorname{dim}_{\mathbb{C}} H^{0}\left(\left.\mathbb{T}_{\boldsymbol{X}}\right|_{x}\right)-\operatorname{dim}_{\mathbb{C}} H^{1}\left(\left.\mathbb{T}_{\boldsymbol{X}}\right|_{x}\right) \\
& =\operatorname{dim}_{\mathbb{C}} H^{0}\left(\left.\mathbb{T}_{\boldsymbol{X}}\right|_{x}\right)-\operatorname{dim}_{\mathbb{C}} H^{1}\left(\left.\mathbb{T}_{\boldsymbol{X}}\right|_{x}\right)+\operatorname{dim}_{\mathbb{C}} H^{2}\left(\left.\mathbb{T}_{\boldsymbol{X}}\right|_{x}\right)=\operatorname{vdim}_{\mathbb{C}} \boldsymbol{X}=n .
\end{aligned}
$$

Here in the second step we use $\operatorname{dim}_{\mathbb{R}} \Pi_{v}=\frac{1}{2} \operatorname{dim}_{\mathbb{R}} H^{1}\left(\left.\mathbb{T}_{\boldsymbol{X}}\right|_{x}\right)$ by $(*)$ and (3.9), in the third that $H^{0}\left(\left.\mathbb{T}_{\boldsymbol{X}}\right|_{x}\right) \cong H^{2}\left(\left.\mathbb{T}_{\boldsymbol{X}}\right|_{x}\right)^{*}$ as $\left(\boldsymbol{X}, \omega_{\boldsymbol{X}}^{*}\right)$ is -2 -shifted symplectic (or -2-shifted presymplectic will do), and in the fourth that $\mathbb{T}_{\boldsymbol{X}}$ is perfect in the interval $[0,2]$ as $\left(\boldsymbol{X}, \omega_{\boldsymbol{X}}^{*}\right)$ is -2-shifted symplectic (or presymplectic).

Equation (3.26) says that the Kuranishi neighbourhood $\left(U, E^{+}, s^{+}, \psi^{+}\right)$has real virtual dimension $\operatorname{dim} U-\operatorname{rank} E^{+}=n=\operatorname{vdim}_{\mathbb{C}} \boldsymbol{X}=\frac{1}{2} \operatorname{vdim}_{\mathbb{R}} \boldsymbol{X}$. Note that this is half the virtual dimension we might have expected, and the real virtual dimension can be odd, even though $\boldsymbol{X}, V, E, s, \ldots$ are all complex.

Here are some important properties of such $U, E^{-}, E^{+}, s^{+}$, proved in $\$ 5$

Theorem 3.7. In the situation of Definition 3.6. with $\boldsymbol{X}, \omega_{\boldsymbol{X}}^{*}, A^{\bullet}, \boldsymbol{\alpha}, V, E, F, s$, $t, \psi$ fixed, we have:

(a) If the conditions in $(*)$ hold at some $v \in s^{-1}(0) \cap U$, then they also hold for all $v^{\prime}$ in an open neighbourhood of $v$ in $s^{-1}(0) \cap U$.

(b) Suppose $C \subseteq V$ is closed, and $\left(U, E^{-}\right)$satisfies condition $(*)$ with $C \subseteq$ $U \subseteq V$. (We allow $C=U=\emptyset$.) Then there exists $\left(\tilde{U}, \tilde{E}^{-}\right)$satisfying $(*)$ with $C \cup s^{-1}(0) \subseteq \tilde{U} \subseteq V$, and an open neighbourhood $U^{\prime}$ of $C$ in $U \cap \tilde{U}$ such that $\left.E^{-}\right|_{U^{\prime}}=\left.\tilde{E}^{-}\right|_{U^{\prime}}$. 
(c) If $\left(U, E^{-}\right)$satisfies $(*)$, the closed subsets $s^{-1}(0) \cap U$ and $\left(s^{+}\right)^{-1}(0)$ in $U \subseteq V$ coincide in an open neighbourhood $U^{\prime}$ of $s^{-1}(0) \cap U$ in $U$. Hence $\left(U^{\prime},\left.E^{-}\right|_{U^{\prime}}\right)$ satisfies condition $(\dagger)$, and $\left(U^{\prime},\left.E^{+}\right|_{U^{\prime}},\left.s^{+}\right|_{U^{\prime}}, \psi^{+}\right)$is a Kuranishi neighbourhood on $X_{\text {an }}$. Thus, we can make $\left(U, E^{-}\right)$satisfying $(*)$ also satisfy $(\dagger)$ by shrinking $U$, without changing $R=\operatorname{Im} \psi$ in $X_{\text {an }}$.

The next example proves Theorem 3.7(c) near $v \in s^{-1}(0) \cap U$ in a special case, when $\left(A^{\bullet}, \omega_{A} \bullet\right)$ is in -2-Darboux form and minimal at $v$. The general case in $\$ 5.3$ is proved by reducing to Example 3.8 .

Example 3.8. Suppose $\left(\boldsymbol{X}, \omega_{\boldsymbol{X}}^{*}\right)$ is a -2 -shifted symplectic derived $\mathbb{C}$-scheme, and $x \in X_{\text {an }}$. Then Theorem 2.10 gives a pair $\left(A^{\bullet}, \omega_{A} \bullet\right)$ in -2-Darboux form and a Zariski open inclusion $\boldsymbol{\alpha}: \operatorname{Spec} A^{\bullet} \hookrightarrow \boldsymbol{X}$ which is minimal at $x \in \operatorname{Im} \boldsymbol{\alpha}$, with $\boldsymbol{\alpha}^{*}\left(\omega_{\boldsymbol{X}}^{*}\right) \simeq \omega_{A} \bullet$ in $\mathcal{A}_{\mathbb{C}}^{2, \text { cl }}\left(\operatorname{Spec} A^{\bullet},-2\right)$.

Example 3.3 describes the data $V, E, F, s, t$ associated to $A^{\bullet}$ in 33.2 , and defines a nondegenerate quadratic form $Q \in H^{0}\left(S^{2} E^{*}\right)$ with $Q(s, s)=0$ using $\omega_{A} \bullet$. As $x \in \operatorname{Im} \boldsymbol{\alpha}$ there is $v \in s^{-1}(0) \subseteq V$ with $\boldsymbol{\alpha}(v)=x$, and $\left(A^{\bullet}, \boldsymbol{\alpha}\right)$ minimal at $x$ means that $\left.\mathrm{d} s\right|_{v}=0$, so that $\left.t\right|_{v}=0$ by (3.13). Thus in (3.9) we have $\operatorname{Ker}\left(\left.t\right|_{v}\right) / \operatorname{Im}\left(\left.\mathrm{d} s\right|_{v}\right)=\left.E\right|_{v}$, identified with $H^{1}\left(\left.\mathbb{T}_{\boldsymbol{X}}\right|_{x}\right)$. Since $\boldsymbol{\alpha}^{*}\left(\omega_{\boldsymbol{X}}^{*}\right) \simeq \omega_{A} \bullet$, the quadratic form $\tilde{Q}_{v}$ on $\operatorname{Ker}\left(\left.t\right|_{v}\right) / \operatorname{Im}\left(\left.\mathrm{d} s\right|_{v}\right)=\left.E\right|_{v}$ in (3.21) is $\left.Q\right|_{v}$.

Given a pair $\left(U, E^{-}\right)$as in Definition 3.6 with $v \in U$, the map $\Pi_{v}$ in (3.24) is just the inclusion $\left.\left.E^{-}\right|_{v} \hookrightarrow E\right|_{v}$. So $(*)$ at $v$ says that $\left.E^{-}\right|_{v}$ is a real vector subspace of $\left.E\right|_{v}$ with $\left.\operatorname{dim}_{\mathbb{R}} E^{-}\right|_{v}=\left.\frac{1}{2} \operatorname{dim}_{\mathbb{R}} E\right|_{v}=\left.\operatorname{dim}_{\mathbb{C}} E\right|_{v}$, such that $\left.\operatorname{Re} Q\right|_{v}$ is negative definite on $\left.E^{-}\right|_{v}$.

As this is an open condition, there exists an open neighbourhood $U^{\prime}$ of $v$ in $U$ such that $\left.\operatorname{Re} Q\right|_{U^{\prime}}$ is negative definite on $\left.E^{-}\right|_{U^{\prime}}$. Define a real vector subbundle $\tilde{E}^{+}$of $\left.E\right|_{U^{\prime}}$ to be the orthogonal subbundle of $\left.E^{-}\right|_{U^{\prime}}$ w.r.t. the nondegenerate real quadratic form $\left.\operatorname{Re} Q\right|_{U^{\prime}}$. Then $\left.E\right|_{U^{\prime}}=\left.\tilde{E}^{+} \oplus E^{-}\right|_{U^{\prime}}$, so we can write $\left.s\right|_{U^{\prime}}=\tilde{s}^{+} \oplus s^{-}$, for $\tilde{s}^{+} \in C^{\infty}\left(\tilde{E}^{+}\right)$and $s^{-} \in C^{\infty}\left(\left.E^{-}\right|_{U^{\prime}}\right)$. The projection $\left.\left.E\right|_{U^{\prime}} \rightarrow E^{+}\right|_{U^{\prime}}=\left.E\right|_{U^{\prime}} /\left.E^{-}\right|_{U^{\prime}}$ restricts to an isomorphism $\left.\tilde{E}^{+} \rightarrow E^{+}\right|_{U^{\prime}}$, which $\left.\operatorname{maps} \tilde{s}^{+} \mapsto s^{+}\right|_{U^{\prime}}$.

Because $\operatorname{Re} Q$ is the real part of a complex form, it has the same number of positive as negative eigenvalues. Thus $\left.\operatorname{Re} Q\right|_{U^{\prime}}$ is positive definite on $\tilde{E}^{+}$. Now

$$
0=\left.\operatorname{Re} Q(s, s)\right|_{U^{\prime}}=\operatorname{Re} Q\left(\tilde{s}^{+}+s^{-}, \tilde{s}^{+}+s^{-}\right)=\operatorname{Re} Q\left(\tilde{s}^{+}, \tilde{s}^{+}\right)+\operatorname{Re} Q\left(s^{-}, s^{-}\right),
$$

using $\operatorname{Re} Q\left(\tilde{s}^{+}, s^{-}\right)=0$ as $\tilde{E}^{+},\left.E^{-}\right|_{U^{\prime}}$ are orthogonal w.r.t. $\left.\operatorname{Re} Q\right|_{U^{\prime}}$.

For each $u \in U^{\prime}$, we now have

$$
\begin{aligned}
& s^{+}(u)=0 \quad \Longleftrightarrow \quad \tilde{s}^{+}(u)=\left.0 \quad \Longleftrightarrow \operatorname{Re} Q\left(\tilde{s}^{+}, \tilde{s}^{+}\right)\right|_{u}=0 \quad \Longleftrightarrow \\
& \left.\operatorname{Re} Q\left(s^{-}, s^{-}\right)\right|_{u}=0 \Longleftrightarrow \tilde{s}^{+}(u)=s^{-}(u)=0 \Longleftrightarrow s(u)=0,
\end{aligned}
$$

using $\left.\tilde{E}^{+} \rightarrow E^{+}\right|_{U^{\prime}}$ an isomorphism mapping $\left.\tilde{s}^{+} \mapsto s^{+}\right|_{U^{\prime}}$ in the first step, $\operatorname{Re} Q$ positive definite on $\tilde{E}^{+}$in the second, (3.27) in the third, $\operatorname{Re} Q$ negative definite on $\left.E^{-}\right|_{U^{\prime}}$ in the fourth, and $\left.s\right|_{U^{\prime}}=\tilde{s}^{+} \oplus s^{-}$in the fifth.

This proves there exists an open neighbourhood $U^{\prime}$ of $v$ in $U$ such that $s^{-1}(0) \cap U^{\prime}=\left(s^{+}\right)^{-1}(0) \cap U^{\prime}$, which is Theorem 3.7(c), except that $U^{\prime}$ is a neighbourhood of $v$ rather than of $s^{-1}(0) \cap U$. 
Remark 3.9. Pairs $\left(U, E^{-}\right)$satisfying $(\dagger)$ will be used to prove our main result, constructing a derived manifold structure $\boldsymbol{X}_{\mathrm{dm}}$ on the complex analytic topological space $X_{\text {an }}$ of a -2 -shifted symplectic derived $\mathbb{C}$-scheme $\left(\boldsymbol{X}, \omega_{\boldsymbol{X}}^{*}\right)$.

Our construction apparently uses less than the full -2 -shifted symplectic structure $\omega_{\boldsymbol{X}}^{*}$ on $\boldsymbol{X}$. In particular, conditions $(*),(\dagger)$ only involve the nondegenerate pairings $\left.\omega_{\boldsymbol{X}}^{0}\right|_{x}$ on $H^{1}\left(\left.\mathbb{T}_{\boldsymbol{X}}\right|_{x}\right)$ in (2.6), which depend only on the presymplectic structure $\omega_{\boldsymbol{X}}^{0}$, not the symplectic structure $\omega_{\boldsymbol{X}}^{*}=\left(\omega_{\boldsymbol{X}}^{0}, \omega_{\boldsymbol{X}}^{1}, \ldots\right)$. The proofs of Theorem $3.7(a),(b)$ in $\$ 5.1-\$ 5.2$ also use only $\omega_{\boldsymbol{X}}^{0}$ rather than $\omega_{\boldsymbol{X}}^{*}$.

However, the proof of Theorem 3.7 (c) in $\$ 5.3$ involves $\omega_{\boldsymbol{X}}^{*}$, as it uses the existence of a minimal -2-Darboux form presentation for $\left(\boldsymbol{X}, \omega_{\boldsymbol{X}}^{*}\right)$ near each $x \in X_{\text {an }}$, as in Theorem 2.10. The authors do not know whether Theorem 3.7(c) holds for -2 -shifted presymplectic $\left(\boldsymbol{X}, \omega_{\boldsymbol{X}}^{0}\right)$ which are not symplectic.

\subsection{Comparing $\left(U_{J}, E_{J}^{-}\right),\left(U_{K}, E_{K}^{-}\right)$under $\Phi_{J K}$}

Section 3.3 discussed how to use standard form charts $\boldsymbol{\alpha}: \operatorname{Spec} A^{\bullet} \rightarrow \boldsymbol{X}$ on $\left(\boldsymbol{X}, \omega_{\boldsymbol{X}}^{*}\right)$ to choose pairs $\left(U, E^{-}\right)$, and so define Kuranishi neighbourhoods $\left(U, E^{+}, s^{+}, \psi^{+}\right)$on $X_{\mathrm{an}}$. We now explain how to pull back such pairs $\left(U_{K}, E_{K}^{-}\right)$ along a quasi-free morphism $\Phi_{J K}: A_{K}^{\bullet} \rightarrow A_{J}^{\bullet}$, and construct coordinate changes between the Kuranishi neighbourhoods $\left(U_{J}, E_{J}^{+}, s_{J}^{+}, \psi_{J}^{+}\right),\left(U_{K}, E_{K}^{+}, s_{K}^{+}, \psi_{K}^{+}\right)$.

Definition 3.10. Let $\left(\boldsymbol{X}, \omega_{\boldsymbol{X}}^{*}\right)$ be a -2 -shifted symplectic derived $\mathbb{C}$-scheme with $\operatorname{vdim}_{\mathbb{C}} \boldsymbol{X}=n$, and suppose $\Phi_{J K}: A_{K}^{\bullet} \rightarrow A_{J}^{\bullet}$ is a quasi-free morphism of standard form cdgas over $\mathbb{C}$ and $\boldsymbol{\alpha}_{J}: \operatorname{Spec} A_{J}^{\bullet} \hookrightarrow \boldsymbol{X}, \boldsymbol{\alpha}_{K}: \operatorname{Spec} A_{K}^{\bullet} \hookrightarrow \boldsymbol{X}$ are Zariski open inclusions such that (3.3) homotopy commutes. Define complex geometric data $V_{J}, E_{J}, F_{J}, s_{J}, t_{J}, \psi_{J}, R_{J}, V_{K}, E_{K}, F_{K}, s_{K}, t_{K}, \psi_{K}, R_{K}, \phi_{J K}, \chi_{J K}$, $\xi_{J K}$ in Definitions 3.2, 3.4, and suppose $R_{J} \neq \emptyset$, so $R_{K} \neq \emptyset$ as $R_{J} \subseteq R_{K} \subseteq X_{\text {an }}$.

Consider pairs $\left(U_{J}, E_{J}^{-}\right)$for $A_{J}^{\bullet}$ and $\left(U_{K}, E_{K}^{-}\right)$for $A_{K}^{\bullet}$ satisfying condition $(*)$ in Definition [3.6. We say that $\left(U_{J}, E_{J}^{-}\right)$and $\left(U_{K}, E_{K}^{-}\right)$are compatible if $\phi_{J K}\left(U_{J}\right) \subseteq U_{K}$ and $\left.\left.\left.\chi_{J K}\right|_{U_{J}}\left(E_{J}^{-}\right) \subseteq \phi_{J K}\right|_{U_{J}} ^{*}\left(E_{K}^{-}\right) \subseteq \phi_{J K}\right|_{U_{J}} ^{*}\left(E_{K}\right)$.

For $\left(U_{J}, E_{J}^{-}\right),\left(U_{K}, E_{K}^{-}\right)$compatible, define a vector bundle morphism $\chi_{J K}^{+}$: $\left.E_{J}^{+} \rightarrow \phi_{J K}\right|_{U_{J}} ^{*}\left(E_{K}^{+}\right)$on $U_{J}$ by the commutative diagram with exact rows

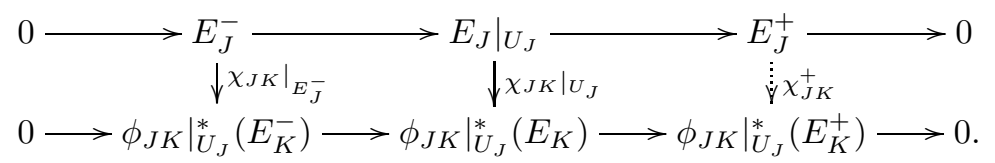

Let $v_{J} \in s_{J}^{-1}(0) \subseteq U_{J} \subseteq V_{J}$ with $\phi_{J K}\left(v_{J}\right)=v_{K} \in s_{K}^{-1}(0) \subseteq U_{K} \subseteq V_{K}$ and $\psi_{J}\left(v_{J}\right)=\psi_{K}\left(v_{K}\right)=x \in X_{\mathrm{an}}$. Consider the diagram, with rows (3.25) for $\left(U_{J}, E_{J}^{-}\right), v_{J}$ and $\left(U_{K}, E_{K}^{-}\right), v_{K}$

$$
\begin{aligned}
0 & \left.\rightarrow H^{0}\left(\left.\mathbb{T}_{\boldsymbol{X}}\right|_{x}\right) \rightarrow T_{v_{J}} U_{J} \underset{\left.\mathrm{d} s_{J}^{+}\right|_{v_{J}}}{\longrightarrow} E_{J}^{+}\right|_{v_{J}} \rightarrow H^{1}\left(\left.\mathbb{T}_{\boldsymbol{X}}\right|_{x}\right) / \operatorname{Im} \Pi_{v_{J}} \rightarrow 0 \\
\quad \text { id }\left.\downarrow \quad \mathrm{d} \phi_{J K}\right|_{v_{J}} \downarrow & \left.\mathrm{d} s_{K}^{+}\right|_{v_{K}} \\
0 & \rightarrow H^{0}\left(\left.\mathbb{T}_{\boldsymbol{X}}\right|_{x}\right) \rightarrow T_{v_{K}} U_{K} \stackrel{v_{v_{K}}}{\longrightarrow} H^{1}\left(\left.\mathbb{T}_{\boldsymbol{X}}\right|_{x}\right) / \operatorname{Im} \Pi_{v_{K}} \rightarrow 0 .
\end{aligned}
$$


Here if we regard $\operatorname{Im} \Pi_{v_{J}}, \operatorname{Im} \Pi_{v_{K}}$ from (3.24) as subspaces of $H^{1}\left(\left.\mathbb{T}_{\boldsymbol{X}}\right|_{x}\right)$ using (3.9), compatibility $\left.\chi_{J K}\left(\left.E_{J}^{-}\right|_{v_{J}}\right) \subseteq E_{K}^{-}\right|_{v_{K}}$ and (3.18) imply that $\operatorname{Im} \Pi_{v_{J}} \subseteq$ $\operatorname{Im} \Pi_{v_{K}}$, so $\operatorname{Im} \Pi_{v_{J}}=\operatorname{Im} \Pi_{v_{K}}$ as they have the same dimension by $(*)$, and the right hand column of (3.28) makes sense. From (3.14), (3.17) and (3.18) we see that (3.28) commutes. Elementary linear algebra then gives an exact sequence

$$
\left.\left.0 \longrightarrow T_{v_{J}} U_{J} \stackrel{\left.\left.\mathrm{d} s_{J}^{+}\right|_{v_{J}} \oplus \mathrm{d} \phi_{J K}\right|_{v_{J}}}{\longrightarrow} T_{v_{K}} E_{K}^{+}\right|_{v_{J}} \oplus \stackrel{-\left.\left.\chi_{J K}^{+}\right|_{v_{J}} \oplus \mathrm{d} s_{K}^{+}\right|_{v_{K}}}{\longrightarrow} E_{K}^{+}\right|_{v_{K}} \rightarrow 0 .
$$

From (3.29) and Definition 2.14, we deduce:

Corollary 3.11. In the situation of Definition 3.10 if $\left(U_{J}, E_{J}^{-}\right)$and $\left(U_{K}, E_{K}^{-}\right)$ are compatible and satisfy $(\dagger)$ then in the sense of 2.5

$$
\left(U_{J},\left.\phi_{J K}\right|_{U_{J}}, \chi_{J K}^{+}\right):\left(U_{J}, E_{J}^{+}, s_{J}^{+}, \psi_{J}\right) \longrightarrow\left(U_{K}, E_{K}^{+}, s_{K}^{+}, \psi_{K}\right)
$$

is a coordinate change of Kuranishi neighbourhoods on $X_{\mathrm{an}}$.

Lemma 3.12. In the situation of Definition 3.10, fix $\left(U_{K}, E_{K}^{-}\right)$satisfying $(*)$ for $A_{K}^{\bullet}, \boldsymbol{\alpha}_{K}$. Set $U_{J K}^{\prime}=\phi_{J K}^{-1}\left(U_{K}\right) \subseteq V_{J}$. Then $E_{J K}^{\prime}:=\left.\chi_{J K}\right|_{U_{J K}^{\prime}} ^{-1}\left(E_{K}^{-}\right)$is a vector subbundle of $\left.E_{J}\right|_{U_{J K}^{\prime}}$, as $\chi_{J K}$ is surjective. Choose a complementary real vector subbundle $E_{J K}^{\prime \prime}$, so that $\left.E_{J}\right|_{U_{J K}^{\prime}}=E_{J K}^{\prime} \oplus E_{J K}^{\prime \prime}$.

Choose a connection $\nabla$ on $E_{J}$, so that $\nabla s_{J}: T V_{J} \rightarrow E_{J}$ is a vector bundle morphism. Now $\operatorname{Ker}\left(\mathrm{d} \phi_{J K}: T V_{J} \rightarrow \phi_{J K}^{*}\left(T V_{K}\right)\right)$ is a vector subbundle of $T V_{J}$, as $\mathrm{d} \phi_{J K}$ is surjective, and $\nabla s_{J}$ is injective on $\operatorname{Ker} \phi_{J K}$ near $s_{J}^{-1}(0)$, so $E_{J K}^{\prime \prime \prime}:=$ $\left(\nabla s_{J}\right)\left[\operatorname{Ker} \mathrm{d} \phi_{J K}\right]$ is a vector subbundle of $E_{J}$ near $s_{J}^{-1}(0)$ in $V_{J}$.

Then $\left(U_{J}, E_{J}^{-}\right)$satisfies $(*)$ for $A_{J}^{\bullet}, \boldsymbol{\alpha}_{J}$ and is compatible with $\left(U_{K}, E_{K}^{-}\right)$if and only if $U_{J}$ is open in $U_{J K}^{\prime}$, and $E_{J K}^{-}$is a vector subbundle of $\left.E_{J K}^{\prime}\right|_{U_{J}}$ satisfying $\left.E_{J}\right|_{U_{J}}=\left.\left.E_{J K}^{-} \oplus E_{J K}^{\prime \prime}\right|_{U_{J}} \oplus E_{J K}^{\prime \prime \prime}\right|_{U_{J}}$ near $s_{J}^{-1}(0) \cap U_{J}$ in $U_{J}$. Alternatively, identifying $E_{J K}^{\prime}$ with $\left.E_{J}\right|_{U_{J K}^{\prime}} / E_{J K}^{\prime \prime}$, this condition may be written as $\left.E_{J K}^{\prime}\right|_{U_{J}}=\left.E_{J K}^{-} \oplus\left[\left(E_{J K}^{\prime \prime} \oplus E_{J K}^{\prime \prime \prime}\right) / E_{J K}^{\prime \prime}\right]\right|_{U_{J}}$ near $s_{J}^{-1}(0) \cap U_{J}$.

Proof. We deduce $\nabla s_{J}$ is injective on Ker $\mathrm{d} \phi_{J K}$ at $v_{J} \in s_{J}^{-1}(0)$ using (3.17), check that $(*)$ for $U_{J}, E_{J}^{-}$is equivalent to $E_{J}=E_{J K}^{-} \oplus E_{J K}^{\prime \prime} \oplus E_{J K}^{\prime \prime \prime}$ at each $v_{J} \in s_{J}^{-1}(0)$, and note that both are open conditions.

Lemma 3.12 shows we can always pullback $\left(U_{K}, E_{K}^{-}\right)$satisfying $(*)$ along submersions $\phi_{J K}: V_{J} \rightarrow V_{K}$ : we just have to choose a complement $E_{J}^{-}$to $\left(E_{J K}^{\prime \prime} \oplus E_{J K}^{\prime \prime \prime}\right) / E_{J K}^{\prime \prime}$ in $E_{J K}^{\prime}$ on some small open neighbourhood $U_{J}$ of $s_{J}^{-1}(0)$ in $U_{J K}^{\prime}$, for instance, the orthogonal complement w.r.t. any metric on $E_{J K}^{\prime}$. By Theorem 3.7(c), making $U_{J}$ smaller, we can suppose $\left(U_{J}, E_{J}^{-}\right)$satisfies $(\dagger)$.

\subsection{Constructing Kuranishi atlases and derived manifolds}

Let $\left(\boldsymbol{X}, \omega_{\boldsymbol{X}}^{*}\right)$ be a -2 -shifted symplectic derived $\mathbb{C}$-scheme with $\operatorname{vdim}_{\mathbb{C}} \boldsymbol{X}=n$ in $\mathbb{Z}$, and write $X_{\text {an }}$ for the complex analytic topological space. Suppose $\boldsymbol{X}$ is separated and $X_{\mathrm{an}}$ is a paracompact topological space. (Paracompactness is 
automatic if $\boldsymbol{X}$ is proper, or quasicompact, or of finite type, or $X_{\text {an }}$ is second countable.) We will construct a Kuranishi atlas on $X_{\mathrm{an}}$, in the sense of $\$ 2.5$.

First choose a family $\left\{\left(A_{i}^{\bullet}, \boldsymbol{\alpha}_{i}\right): i \in I\right\}$, where $A_{i}^{\bullet} \in \mathbf{c d g a}_{\mathbb{C}}$ is a standard form cdga, and $\boldsymbol{\alpha}_{i}: \operatorname{Spec} A_{i}^{\bullet} \hookrightarrow \boldsymbol{X}$ a Zariski open inclusion in $\mathbf{d S c h}_{\mathbb{C}}$ for each $i$ in $I$, an indexing set, such that $\left\{R_{i}:=\left(\operatorname{Im} \boldsymbol{\alpha}_{i}\right)_{\text {an }}: i \in I\right\}$ is an open cover of the complex analytic topological space $X_{\text {an }}$. This is possible by Theorem 2.5. If $\boldsymbol{X}$ is quasicompact (since $\boldsymbol{X}$ is locally of finite type, this is equivalent to $\boldsymbol{X}$ being of finite type) then we can take $I$ to be finite.

Apply Theorem 3.1 to get data $A_{J}^{\bullet} \in \mathbf{c d g a}_{\mathbb{C}}, \boldsymbol{\alpha}_{J}: \operatorname{Spec} A_{J}^{\bullet} \hookrightarrow \boldsymbol{X}$ for finite $\emptyset \neq J \subseteq I$ and quasi-free $\Phi_{J K}: A_{K}^{\bullet} \rightarrow A_{J}^{\bullet}$, for all finite $\emptyset \neq K \subseteq J \subseteq I$.

Use the notation of 33.2 to rewrite $A_{J}^{\bullet}, \Phi_{J K}$ in terms of complex geometry. As in Corollary 3.5, this gives data $V_{J}, E_{J}, F_{J}, s_{J}, t_{J}, \psi_{J}, R_{J}$ for all finite $\emptyset \neq J \subseteq I$, and $\phi_{J K}, \chi_{J K}, \xi_{J K}$ for all finite $\emptyset \neq K \subseteq J \subseteq I$.

For brevity we write $A=\{J: \emptyset \neq J \subseteq I, J$ is finite $\}$. The proof of the next result in 6.1 is based on McDuff and Wehrheim [28, Lem. 7.1.7].

Proposition 3.13. Suppose $Z$ is a paracompact, Hausdorff topological space and $\left\{R_{i}: i \in I\right\}$ an open cover of $Z$. Then we can choose closed subsets $C_{J} \subseteq Z$ for all finite $\emptyset \neq J \subseteq I$, satisfying:

(i) $C_{J} \subseteq \bigcap_{i \in J} R_{i}$ for all $J$.

(ii) Each $z \in Z$ has an open neighbourhood $U_{z} \subseteq Z$ with $U_{z} \cap C_{J} \neq \emptyset$ for only finitely many $J$.

(iii) $C_{J} \cap C_{K} \neq \emptyset$ only if $J \subseteq K$ or $K \subseteq J$.

(iv) $\bigcup_{\emptyset \neq J \subseteq I \text { finite }} C_{J}=Z$.

In our case, $X_{\mathrm{an}}$ is Hausdorff and second countable. It is also locally compact, as it is locally homeomorphic to closed subsets $s_{J}^{-1}(0)$ of complex manifolds $V_{J}$. But Hausdorff, locally compact and second countable imply that $X$ is paracompact and normal. Thus Proposition 3.13 applies to $Z=X_{\text {an }}$ with the open cover $\left\{R_{i}: i \in I\right\}$, and we can choose closed subsets $C_{J} \subseteq R_{J}=\bigcap_{i \in J} R_{i} \subseteq$ $X_{\text {an }}$ for all $J \in A$ satisfying conditions (i)-(iv).

The next proposition, proved in $\$ 6.2$ using Theorem 3.7 and Lemma 3.12 , chooses pairs $\left(U_{J}, E_{J}^{-}\right)$satisfying $(\dagger)$, as in $\$ 3.3$, with $\left(U_{J}, E_{J}^{-}\right),\left(U_{K}, E_{K}^{-}\right)$compatible near $C_{J} \cap C_{K}$ under the quasi-free morphism $\Phi_{J K}: A_{K}^{\bullet} \rightarrow A_{J}^{\bullet}$.

Proposition 3.14. In the situation above, we can choose $\left(U_{J}, E_{J}^{-}\right)$satisfying condition ( $\dagger$ ) for $V_{J}, E_{J}, \ldots$ for each $J \in A$, such that $\psi_{J}^{-1}\left(C_{J}\right) \subseteq U_{J} \subseteq V_{J}$, and setting $S_{J}=\psi_{J}\left(s_{J}^{-1}(0) \cap U_{J}\right)$ so that $S_{J}$ is an open neighbourhood of $C_{J}$ in $X_{\text {an }}$, then for all $J, K \in A$, we have $S_{J} \cap S_{K} \neq \emptyset$ only if $J \subseteq K$ or $K \subseteq J$, and if $K \subsetneq J$ then there exists open $U_{J K} \subseteq U_{J}$ with $s_{J}^{-1}(0) \cap U_{J K}=\psi_{J}^{-1}\left(S_{J} \cap S_{K}\right)$ such that $\left(U_{J K},\left.E_{J}^{-}\right|_{U_{J K}}\right)$ is compatible with $\left(U_{K}, E_{K}^{-}\right)$, in the sense of 3.4 .

We can now prove two of the central results of this paper. 
Theorem 3.15. Let $\left(\boldsymbol{X}, \omega_{\boldsymbol{X}}^{*}\right)$ be a-2-shifted symplectic derived $\mathbb{C}$-scheme with complex virtual dimension $\operatorname{vdim}_{\mathbb{C}} \boldsymbol{X}=n$ in $\mathbb{Z}$, and write $X_{\text {an }}$ for the set of $\mathbb{C}$ points of $X=t_{0}(\boldsymbol{X})$ with the complex analytic topology. Suppose that $\boldsymbol{X}$ is separated, and $X_{\mathrm{an}}$ is a paracompact topological space. Then we can construct a Kuranishi atlas $\mathcal{K}$ on $X_{\text {an }}$ of real dimension n, in the sense of $\$ 2.5$. If $\boldsymbol{X}$ is quasicompact (equivalently, of finite type) then we can take $\mathcal{K}$ to be finite.

Proof. In the discussion from the beginning of 83.5 up to Proposition 3.14, we have constructed the following data:

(i) A Hausdorff, paracompact topological space $X_{\text {an }}$.

(ii) An indexing set $I$, where we write $A=\{J: \emptyset \neq J \subseteq I, J$ is finite $\}$.

(iii) An open cover $\left\{S_{J}: J \in A\right\}$ of $X_{\text {an }}$, such that $S_{J} \cap S_{K} \neq \emptyset$ for $J, K \in A$ only if $J \subseteq K$ or $K \subseteq J$.

(iv) For each $J \in A$, a Kuranishi neighbourhood $\left(U_{J}, E_{J}^{+}, s_{J}^{+}, \psi_{J}^{+}\right)$on $X_{\text {an }}$ with $\operatorname{dim} U_{J}-\operatorname{rank} E_{J}^{+}=n$, constructed as in $\$ 3.3$ from $\left(U_{J}, E_{J}^{-}\right)$satisfying $(\dagger)$, with $\operatorname{Im} \psi_{J}^{+}=S_{J} \subseteq X_{\text {an }}$.

(v) For all $J, K \in A$ with $K \subsetneq J$, a coordinate change of Kuranishi neighbourhoods over $S_{J} \cap S_{K}$, as in Corollary 3.11 .

$$
\left(U_{J K},\left.\phi_{J K}\right|_{U_{J K}}, \chi_{J K}^{+}\right):\left(U_{J}, E_{J}^{+}, s_{J}^{+}, \psi_{J}^{+}\right) \longrightarrow\left(U_{K}, E_{K}^{+}, s_{K}^{+}, \psi_{K}^{+}\right),
$$

since $\left(U_{J K},\left.E_{J}^{-}\right|_{U_{J K}}\right)$ is compatible with $\left(U_{K}, E_{K}^{-}\right)$.

(vi) For all $J, K, L \in A$ with $L \subsetneq K \subsetneq J$, Corollary 3.5 implies that $\phi_{J L}=$ $\phi_{K L} \circ \phi_{J K}$ and $\chi_{J L}^{+}=\phi_{J K}^{*}\left(\chi_{K L}^{+}\right) \circ \chi_{J K}^{+}$on $U_{J K} \cap U_{J L} \cap \phi_{J K}^{-1}\left(U_{K L}\right)$.

All this data is a Kuranishi atlas $\mathcal{K}$ in the sense of Definition 2.15] where the partial order $\prec$ on $A$ is $J \prec K$ if $K \subsetneq J$. If $\boldsymbol{X}$ is quasicompact then we can take $I$ finite, so $A$ and $\mathcal{K}$ are finite.

Combining Theorems 2.18 and 3.15 yields:

Theorem 3.16. Let $\left(\boldsymbol{X}, \omega_{\boldsymbol{X}}^{*}\right)$ be a-2-shifted symplectic derived $\mathbb{C}$-scheme with complex virtual dimension $\operatorname{vdim}_{\mathbb{C}} \boldsymbol{X}=n$ in $\mathbb{Z}$, and write $X_{\text {an }}$ for the set of $\mathbb{C}$ points of $X=t_{0}(\boldsymbol{X})$ with the complex analytic topology. Suppose that $\boldsymbol{X}$ is separated, so that $X_{\mathrm{an}}$ is Hausdorff, and also that $X_{\mathrm{an}}$ is a second countable topological space, which holds if and only if $X$ admits a Zariski open cover $\left\{X_{c}: c \in C\right\}$ with $C$ countable and each $X_{c}$ a finite type $\mathbb{C}$-scheme.

Then we can make the topological space $X_{\mathrm{an}}$ into a derived manifold $\boldsymbol{X}_{\mathrm{dm}}$ with real virtual dimension $\operatorname{vdim}_{\mathbb{R}} \boldsymbol{X}_{\mathrm{dm}}=n$, in any of the senses (a) Joyce's $m$ Kuranishi spaces mKur [22, §4.7], (b) Joyce's d-manifolds dMan [18 20, (c)

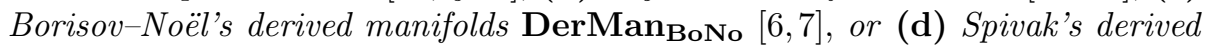
manifolds DerMan $\mathbf{S p i}_{\mathbf{S 1}}$, all discussed in $\$ 2.6$.

We will discuss the dependence of $\boldsymbol{X}_{\mathrm{dm}}$ on choices made in the constructions in 33.6. Note that $\boldsymbol{X}_{\mathrm{dm}}$ in Theorem 3.16 has dimension $\operatorname{vdim}_{\mathbb{R}} \boldsymbol{X}_{\mathrm{dm}}=$ $\operatorname{vdim}_{\mathbb{C}} \boldsymbol{X}=\frac{1}{2} \operatorname{vdim}_{\mathbb{R}} \boldsymbol{X}$, which is exactly half what we might have expected. 


\subsection{Orientations, bordism classes, and virtual classes}

Work in the situation of Theorems 3.15 and 3.16, so that we have a -2 -shifted symplectic derived $\mathbb{C}$-scheme $\left(\boldsymbol{X}, \omega_{\boldsymbol{X}}^{*}\right)$ with complex analytic topological space $X_{\mathrm{an}}$, a Kuranishi atlas $\mathcal{K}$ on $X_{\mathrm{an}}$, and a derived manifold $\boldsymbol{X}_{\mathrm{dm}}$. The next proposition, proved in $\sqrt{6.3}$. justifies our notions of orientation in $92.4-92.6$.

Proposition 3.17. In the situation of Theorems 3.15 and 3.16, there are canonical 1-1 correspondences between:

(a) Orientations on $\left(\boldsymbol{X}, \omega_{\boldsymbol{X}}^{*}\right)$ in the sense of 2.4

(b) Orientations on $\left(X_{\mathrm{an}}, \mathcal{K}\right)$ in the sense of 2.5 and

(c) Orientations on $\boldsymbol{X}_{\mathrm{dm}}$ in the sense of $\$ 2.6 .2$.

Next we consider how the derived manifold $\boldsymbol{X}_{\mathrm{dm}}$ in Theorem 3.16 depends on choices made in the construction. Once we have chosen the Kuranishi atlas $\mathcal{K}$ in Theorem 3.15. Theorem 2.18 shows that $\boldsymbol{X}_{\mathrm{dm}}$ is determined uniquely up to equivalence in its 2 - or $\infty$-category. However, constructing $\mathcal{K}$ involves many arbitrary choices, and the next proposition, proved in 6.4 using the material of 3.7. explains how $\boldsymbol{X}_{\mathrm{dm}}$ depends on these.

Proposition 3.18. In the situation of Theorem 3.16 for $\left(\boldsymbol{X}, \omega_{\boldsymbol{X}}^{*}\right), n$ fixed, the derived manifold $\boldsymbol{X}_{\mathrm{dm}}$ depends on choices made in the construction only up to bordisms of derived manifolds which fix the underlying topological space $X_{\mathrm{an}}$.

That is, if $\boldsymbol{X}_{\mathrm{dm}}, \boldsymbol{X}_{\mathrm{dm}}^{\prime}$ are possible derived manifolds in Theorem 3.16, then we can construct a derived manifold with boundary $\boldsymbol{W}_{\mathrm{dm}}$ with topological space $X_{\mathrm{an}} \times[0,1]$ and $\mathrm{vdim} \boldsymbol{W}_{\mathrm{dm}}=n+1$, and an equivalence of derived manifolds $\partial \boldsymbol{W}_{\mathrm{dm}} \simeq \boldsymbol{X}_{\mathrm{dm}} \amalg \boldsymbol{X}_{\mathrm{dm}}^{\prime}$, topologically identifying $\boldsymbol{X}_{\mathrm{dm}}$ with $X_{\mathrm{an}} \times\{0\}$ and $\boldsymbol{X}_{\mathrm{dm}}^{\prime}$ with $X_{\mathrm{an}} \times\{1\}$. We regard $\boldsymbol{W}_{\mathrm{dm}}$ as a bordism from $\boldsymbol{X}_{\mathrm{dm}}$ to $\boldsymbol{X}_{\mathrm{dm}}^{\prime}$.

This bordism $\boldsymbol{W}_{\mathrm{dm}}$ is compatible with orientations in Proposition 3.17. That is, given an orientation on $\left(\boldsymbol{X}, \omega_{\boldsymbol{X}}^{*}\right)$, we get natural orientations on $\boldsymbol{X}_{\mathrm{dm}}, \boldsymbol{X}_{\mathrm{dm}}^{\prime}$, $\boldsymbol{W}_{\mathrm{dm}}$, and an equivalence of oriented derived manifolds $\partial \boldsymbol{W}_{\mathrm{dm}} \simeq-\boldsymbol{X}_{\mathrm{dm}} \amalg \boldsymbol{X}_{\mathrm{dm}}^{\prime}$, where $-\boldsymbol{X}_{\mathrm{dm}}$ is $\boldsymbol{X}_{\mathrm{dm}}$ with the opposite orientation.

Combining this with material in 2.6.4 2.6.5 yields:

Corollary 3.19. Suppose $\left(\boldsymbol{X}, \omega_{\boldsymbol{X}}^{*}\right)$ is a proper -2-shifted symplectic derived $\mathbb{C}$ scheme, with $\operatorname{vdim}_{\mathbb{C}} \boldsymbol{X}=n$, and with an orientation in the sense of $\$ 2.4$. Then Theorem 3.16 constructs a compact derived manifold $\boldsymbol{X}_{\mathrm{dm}}$ with $\operatorname{vdim}_{\mathbb{R}} \boldsymbol{X}_{\mathrm{dm}}=$ $n$, and Proposition 3.17 defines an orientation on $\boldsymbol{X}_{\mathrm{dm}}$.

Although $\boldsymbol{X}_{\mathrm{dm}}$ depends on arbitrary choices, the d-bordism class $\left[\boldsymbol{X}_{\mathrm{dm}}\right]_{\mathrm{dbo}}$ in $B_{n}(*)$ from $\$ 2.6 .4$ and the virtual class $\left[\boldsymbol{X}_{\mathrm{dm}}\right]_{\mathrm{virt}}$ in $H_{n}\left(X_{\mathrm{an}} ; \mathbb{Z}\right)$ from $\$ 2.6 .5$ are independent of these, and depend only on $\left(\boldsymbol{X}, \omega_{\boldsymbol{X}}^{*}\right)$ and its orientation.

\subsection{Working relative to a smooth base $\mathbb{C}$-scheme $Z$}

Let $Z=\operatorname{Spec} B$ be a smooth classical affine $\mathbb{C}$-scheme, which we now assume is connected. Then the set $Z_{\text {an }}$ of $\mathbb{C}$-points of $Z$ is a complex manifold, and 
hence a real manifold. In this section we will show that all of $33.1-33.6$ also works relatively over the base $Z$. To do this, we will need a notion of a family $\left(\boldsymbol{\pi}: \boldsymbol{X} \rightarrow Z, \omega_{\boldsymbol{X} / Z}\right)$ of -2 -shifted symplectic derived $\mathbb{C}$-schemes over the base $Z$.

To understand the next definition, recall from Remark 3.9 that if $\left(\boldsymbol{X}, \omega_{\boldsymbol{X}}^{*}\right)$ is -2 -shifted symplectic, then the derived manifold $\boldsymbol{X}_{\mathrm{dm}}$ constructed in $\$ 3.5$ does not depend on the whole sequence $\omega_{\boldsymbol{X}}^{*}=\left(\omega_{\boldsymbol{X}}^{0}, \omega_{\boldsymbol{X}}^{1}, \ldots\right)$, but only on the nondegenerate pairings $\left.\omega_{\boldsymbol{X}}^{0}\right|_{x}$ on $H^{1}\left(\left.\mathbb{T}_{\boldsymbol{X}}\right|_{x}\right)$ for $x \in X_{\text {an }}$, and therefore only on the cohomology class $\left[\omega_{\boldsymbol{X}}^{0}\right] \in H^{-2}\left(\mathbb{L}_{\boldsymbol{X}}\right)$. We require that choices of $\omega_{\boldsymbol{X}}^{1}, \omega_{\boldsymbol{X}}^{2}, \ldots$ should exist (they are needed to apply Theorem 2.10, which is used in the proof of Theorem 3.7(c)), but $\boldsymbol{X}_{\mathrm{dm}}$ does not depend on them.

Definition 3.20. Let $\boldsymbol{X}$ be a derived $\mathbb{C}$-scheme, $Z=\operatorname{Spec} B$ a smooth, connected, classical affine $\mathbb{C}$-scheme, and $\boldsymbol{\pi}: \boldsymbol{X} \rightarrow Z$ a morphism. A family of -2-shifted symplectic structures on $\boldsymbol{X} / Z$ is $\left[\omega_{\boldsymbol{X} / Z}\right] \in H^{-2}\left(\mathbb{L}_{\boldsymbol{X} / Z}\right)$, such that for each $z \in Z_{\text {an }}$, writing $\boldsymbol{X}^{z}=\boldsymbol{\pi}^{-1}(z)=\boldsymbol{X} \times{ }_{\boldsymbol{\pi}, Z, z}^{h} *$ for the fibre of $\boldsymbol{\pi}$ over $z$ and $\left.\left[\omega_{\boldsymbol{X} / Z}\right]\right|_{\boldsymbol{X}^{z}} \in H^{-2}\left(\mathbb{L}_{\boldsymbol{X}^{z}}\right)$ for the restriction of $\left[\omega_{\boldsymbol{X} / Z}\right]$ to $\boldsymbol{X}^{z}$, then there should exist a -2 -shifted symplectic structure $\omega_{\boldsymbol{X}^{z}}^{*}=\left(\omega_{\boldsymbol{X}^{z}}^{0}, \omega_{\boldsymbol{X}^{z}}^{1}, \ldots\right)$ on $\boldsymbol{X}^{z}$ such that $\left.\left[\omega_{\boldsymbol{X} / Z}\right]\right|_{\boldsymbol{X}^{z}}=\left[\omega_{\boldsymbol{X}^{z}}^{0}\right]$ in $H^{-2}\left(\mathbb{L}_{\boldsymbol{X}^{z}}\right)$.

That is, a family of -2 -shifted symplectic structures on $\boldsymbol{X} / Z$ is a -2 -shifted relative 2 -form $\left[\omega_{\boldsymbol{X} / Z}\right]$ on $\boldsymbol{X} / Z$, which on each fibre $\boldsymbol{X}^{z}$ extends to a closed 2 -form which is -2 -shifted symplectic. We will explain how to extend the arguments of $33.3-3.6$ to the relative case. Here is the analogue of Definition 3.6.

Definition 3.21. Let $\boldsymbol{X}$ be a derived $\mathbb{C}$-scheme, $Z=\operatorname{Spec} B$ a smooth, classical, affine $\mathbb{C}$-scheme of pure dimension, $\boldsymbol{\pi}: \boldsymbol{X} \rightarrow Z$ a morphism, and $\left[\omega_{\boldsymbol{X} / Z}\right]$ in $H^{-2}\left(\mathbb{L}_{\boldsymbol{X} / Z}\right)$ a family of -2 -shifted symplectic structures on $\boldsymbol{X} / Z$. Write $\operatorname{dim}_{\mathbb{C}} Z=k$ and $\operatorname{vdim}_{\mathbb{C}} \boldsymbol{X}=n+k$. Suppose $A^{\bullet} \in \mathbf{c d g a}_{\mathbb{C}}$ is of standard form, $\boldsymbol{\alpha}: A^{\bullet} \hookrightarrow \boldsymbol{X}$ is a Zariski open inclusion, and $\beta: B \rightarrow A^{0}$ is a smooth morphism of $\mathbb{C}$-algebras, such that (3.10) homotopy commutes. Define complex geometric data $V, \tau, E, F, s, t$ and $\psi: s^{-1}(0) \stackrel{\cong}{\longrightarrow} R \subseteq X_{\text {an }}$ as in Definition 3.2 and suppose $R \neq \emptyset$. Then for each $v \in s^{-1}(0)$ with $\psi(v)=x \in X_{\text {an }}$ and $\tau(v)=\pi(x)=z \in Z_{\mathrm{an}}$, equation (3.12) gives an isomorphism from a vector space depending on $V, \tau, Z_{\text {an }}, E, F, s, t, \tau$ at $v$ to $H^{1}\left(\left.\mathbb{T}_{\boldsymbol{X} / Z}\right|_{x}\right)$.

As in (2.6), the relative 2 -form $\left[\omega_{\boldsymbol{X} / Z}\right]$ induces a pairing

$$
Q_{x}:=\left.\omega_{\boldsymbol{X} / Z}^{0}\right|_{x} \cdot: H^{1}\left(\left.\mathbb{T}_{\boldsymbol{X} / Z}\right|_{x}\right) \times H^{1}\left(\left.\mathbb{T}_{\boldsymbol{X} / Z}\right|_{x}\right) \longrightarrow \mathbb{C},
$$

which is nondegenerate as under the equivalence $\left.\left.\mathbb{T}_{\boldsymbol{X} / Z}\right|_{x} \simeq \mathbb{T}_{\boldsymbol{X}^{z}}\right|_{x}, Q_{x}$ is identified with the pairing induced by a -2 -shifted symplectic form $\omega_{\boldsymbol{X}^{z}}^{*}$ on $\boldsymbol{X}^{z}$, as in Definition 3.20, Define

$$
\tilde{Q}_{v}: \frac{\operatorname{Ker}\left(\left.t\right|_{v}:\left.\left.E\right|_{v} \rightarrow F\right|_{v}\right)}{\operatorname{Im}\left(\left.\mathrm{d} s\right|_{v}:\left.T_{v}\left(V / Z_{\text {an }}\right) \rightarrow E\right|_{v}\right)} \times \frac{\operatorname{Ker}\left(\left.t\right|_{v}:\left.\left.E\right|_{v} \rightarrow F\right|_{v}\right)}{\operatorname{Im}\left(\left.\mathrm{d} s\right|_{v}:\left.T_{v}\left(V / Z_{\text {an }}\right) \rightarrow E\right|_{v}\right)} \longrightarrow \mathbb{C}
$$

to be the nondegenerate complex quadratic form identified with $Q_{x}$ in (3.30) by the isomorphism $H^{1}\left(\left.\mathbb{T}_{\boldsymbol{\alpha}}\right|_{v}\right)$ in (3.12). 
Consider pairs $\left(U, E^{-}\right)$, where $U \subseteq V$ is open and $E^{-}$is a real vector subbundle of $\left.E\right|_{U}$. Given such $\left(U, E^{-}\right)$, we write $E^{+}=\left.E\right|_{U} / E^{-}$for the quotient vector bundle over $U$, and $s^{+} \in C^{\infty}\left(E^{+}\right)$for the image of $\left.s\right|_{U}$ under the projection $\left.E\right|_{U} \rightarrow E^{+}$, and $\psi^{+}:=\left.\psi\right|_{s^{-1}(0) \cap U}: s^{-1}(0) \cap U \rightarrow X_{\text {an }}$. We say that $\left(U, E^{-}\right)$satisfies condition $(*)$ if:

(*) For each $v \in s^{-1}(0) \cap U$, we have

$$
\begin{aligned}
\left.\operatorname{Im}\left(\left.\mathrm{d} s\right|_{v}:\left.T_{v}\left(V / Z_{\text {an }}\right) \rightarrow E\right|_{v}\right) \cap E^{-}\right|_{v} & =\{0\} & & \text { in }\left.E\right|_{v}, \\
\left.t\right|_{v}\left(\left.E^{-}\right|_{v}\right) & =\left.t\right|_{v}\left(\left.E\right|_{v}\right) & & \text { in }\left.F\right|_{v},
\end{aligned}
$$

and the natural real linear map

$$
\Pi_{v}:\left.E^{-}\right|_{v} \cap \operatorname{Ker}\left(\left.t\right|_{v}:\left.\left.E\right|_{v} \rightarrow F\right|_{v}\right) \rightarrow \frac{\operatorname{Ker}\left(\left.t\right|_{v}:\left.\left.E\right|_{v} \rightarrow F\right|_{v}\right)}{\operatorname{Im}\left(\left.\mathrm{d} s\right|_{v}:\left.T_{v}\left(V / Z_{\text {an }}\right) \rightarrow E\right|_{v}\right)},
$$

which is injective by (3.32), has image $\operatorname{Im} \Pi_{v}$ a real vector subspace of dimension exactly half the real dimension of $\operatorname{Ker}\left(\left.t\right|_{v}\right) / \operatorname{Im}\left(\left.\mathrm{d} s\right|_{v}\right)$, and the real quadratic form $\operatorname{Re} \tilde{Q}_{v}$ on $\operatorname{Ker}\left(\left.t\right|_{v}\right) / \operatorname{Im}\left(\left.\mathrm{d} s\right|_{v}\right)$ from (3.31) restricts to a negative definite real quadratic form on $\operatorname{Im} \Pi_{v}$.

We say $\left(U, E^{-}\right)$satisfies condition $(\dagger)$ if:

(†) $\left(U, E^{-}\right)$satisfies condition $(*)$ and $s^{-1}(0) \cap U=\left(s^{+}\right)^{-1}(0) \subseteq U$.

Then $\left(U, E^{+}, s^{+}, \psi^{+}\right)$is a Kuranishi neighbourhood on $X_{\text {an }}$.

Observe that if $v \in s^{-1}(0) \cap U$ with $\psi(v)=x \in X_{\text {an }}$ then using (3.11) (3.12) and (3.32) - (3.34) we find as for (3.25) that there is an exact sequence

$$
\left.0 \rightarrow H^{0}\left(\left.\mathbb{T}_{\boldsymbol{X} / Z}\right|_{x}\right) \rightarrow T_{v}\left(V / Z_{\text {an }}\right) \rightarrow E^{+}\right|_{v} \rightarrow H^{1}\left(\left.\mathbb{T}_{\boldsymbol{X} / Z}\right|_{x}\right) / \operatorname{Im} \Pi_{v} \rightarrow 0 .
$$

Hence as for (3.26) we have

$$
\begin{aligned}
\operatorname{dim}_{\mathbb{R}} U & -\operatorname{dim}_{\mathbb{R}} Z_{\text {an }}-\operatorname{rank}_{\mathbb{R}} E^{+} \\
& =\operatorname{dim}_{\mathbb{R}} H^{0}\left(\left.\mathbb{T}_{\boldsymbol{X} / Z}\right|_{x}\right)-\operatorname{dim}_{\mathbb{R}} H^{1}\left(\left.\mathbb{T}_{\boldsymbol{X} / Z}\right|_{x}\right)+\operatorname{dim}_{\mathbb{R}} \operatorname{Im}_{\Pi_{v}} \\
& =2 \operatorname{dim}_{\mathbb{C}} H^{0}\left(\left.\mathbb{T}_{\boldsymbol{X} / Z}\right|_{x}\right)-\operatorname{dim}_{\mathbb{C}} H^{1}\left(\left.\mathbb{T}_{\boldsymbol{X} / Z}\right|_{x}\right) \\
& =\operatorname{dim}_{\mathbb{C}} H^{0}\left(\left.\mathbb{T}_{\boldsymbol{X} / Z}\right|_{x}\right)-\operatorname{dim}_{\mathbb{C}} H^{1}\left(\left.\mathbb{T}_{\boldsymbol{X} / Z}\right|_{x}\right)+\operatorname{dim}_{\mathbb{C}} H^{2}\left(\left.\mathbb{T}_{\boldsymbol{X} / Z}\right|_{x}\right) \\
& =\operatorname{vdim}_{\mathbb{C}} \boldsymbol{X}-\operatorname{dim}_{\mathbb{C}} Z=n .
\end{aligned}
$$

Thus the Kuranishi neighbourhood $\left(U, E^{+}, s^{+}, \psi^{+}\right)$has virtual dimension

$$
\operatorname{dim} U-\operatorname{rank} E^{+}=n+2 k=\frac{1}{2}\left(\operatorname{vdim}_{\mathbb{R}} \boldsymbol{X}-\operatorname{dim}_{\mathbb{R}} Z_{\text {an }}\right)+\operatorname{dim}_{\mathbb{R}} Z_{\text {an }},
$$

which is the real dimension of the base $Z_{\text {an }}$, plus half the real virtual dimension of the fibres $\boldsymbol{X}^{z}$.

Note that essentially the only important difference between Definitions 3.6 and 3.21 is that $T_{v} V$ in equations (3.21), (3.22), (3.24) is replaced by $T_{v}\left(V / Z_{\text {an }}\right)$ in equations (3.31), (3.32), and (3.34). 
Theorem 3.22. Theorem 3.7 holds with Definition 3.21 in place of Definition 3.6 .

Proof. In the proofs of Theorem 3.7(a),(b) in $\$ 5.1$ \$5.2 we replace $\left.\mathrm{d} s\right|_{v}: T_{v} V \rightarrow$ $\left.E\right|_{v}$ by $\left.\mathrm{d} s\right|_{v}:\left.T_{v}\left(V / Z_{\text {an }}\right) \rightarrow E\right|_{v}$ throughout, and no other changes are needed.

For part (c), fix $z \in Z_{\text {an }}$, so that Definition 3.20 gives a -2 -shifted symplectic derived $\mathbb{C}$-scheme $\left(\boldsymbol{X}^{z}, \omega_{\boldsymbol{X}^{z}}^{*}\right)$ with $\left.\left[\omega_{\boldsymbol{X} / Z}\right]\right|_{\boldsymbol{X}^{z}}=\left[\omega_{\boldsymbol{X}^{z}}^{0}\right]$ in $H^{-2}\left(\mathbb{L}_{\boldsymbol{X}^{z}}\right)$. Consider the complex submanifolds $V^{z}=\tau^{-1}(z)$ in $V$ and $U^{z}=U \cap V^{z}$ in $U$, and write $E^{z}, F^{z}, s^{z}, t^{z}$ for the restrictions of $E, F, s, t$ to $V^{z}$, and $E^{ \pm z}, s^{+z}, \psi^{+z}$ for the restrictions of $E^{ \pm}, s^{+}, \psi^{+}$to $U^{z}$. Then $\left(\boldsymbol{X}^{z}, \omega_{\boldsymbol{X}^{z}}^{*}\right), V^{z}, E^{z}, \ldots$ satisfy Definition 3.6. so Theorem 3.7(c) shows that $\left(s^{z}\right)^{-1}(0) \cap U^{z}$ and $\left(s^{+z}\right)^{-1}(0)$ coincide near $\left(s^{z}\right)^{-1}(0) \cap U^{z}$ in $U^{z}$. Hence $\left(s^{-1}(0) \cap U\right) \cap \tau^{-1}(z)$ and $\left(\left(s^{+}\right)^{-1}(0)\right) \cap \tau^{-1}(z)$ coincide near $\left(s^{-1}(0) \cap U\right) \cap \tau^{-1}(z)$ in $U$. As this holds for all $z \in Z_{\text {an }}, s^{-1}(0) \cap U$ and $\left(s^{+}\right)^{-1}(0)$ coincide near $s^{-1}(0) \cap U$ in $U$, and the theorem follows.

When we extend 33.4 to the relative case, in the analogue of Definition 3.10 we also include data $\boldsymbol{\pi}: \boldsymbol{X} \rightarrow Z=\operatorname{Spec} B$ and smooth $\beta_{J}: B \rightarrow A_{J}^{0}$, $\beta_{K}: B \rightarrow A_{K}^{0}$ with $\beta_{J}=\Phi_{J K} \circ \beta_{K}$ and (3.2) homotopy commuting for $J, K$. We obtain an analogue of (3.28) with rows (3.35) rather than (3.25), and so as for (3.29) we get an exact sequence

$$
\left.0 \rightarrow T_{v_{J}}\left(U_{J} / Z_{\mathrm{an}}\right) \stackrel{\left.\left.\mathrm{d} s_{J}^{+}\right|_{v_{J}} \oplus \mathrm{d} \phi_{J K}\right|_{v_{J}}}{\longrightarrow} T_{v_{K}}\left(\left.U_{K}^{+}\right|_{v_{J}} \oplus Z_{\mathrm{an}}\right) \stackrel{-\left.\left.\chi_{J K}^{+}\right|_{v_{J}} \oplus \mathrm{d} s_{K}^{+}\right|_{v_{K}}}{\longrightarrow} E_{K}^{+}\right|_{v_{K}} \rightarrow 0 .
$$

But by taking the direct sum of this with id $: T_{z} Z_{\text {an }} \rightarrow T_{z} Z_{\text {an }}$ in the second and third positions, we see that this implies (3.29) is exact, and the analogue of Corollary 3.11 follows. The relative analogue of Lemma 3.12, in which we replace $T V_{J}, T V_{K}$ by $T\left(V_{J} / Z_{\text {an }}\right), T\left(V_{K} / Z_{\text {an }}\right)$, is immediate.

For 3.5, we prove the following relative analogue of Theorem 3.15

Theorem 3.23. Let $\boldsymbol{X}$ be a separated derived $\mathbb{C}$-scheme, $Z=\operatorname{Spec} B$ a smooth, connected, classical affine $\mathbb{C}$-scheme, $\boldsymbol{\pi}: \boldsymbol{X} \rightarrow Z$ a morphism, and $\left[\omega_{\boldsymbol{X} / Z}\right]$ a family of -2 -shifted symplectic structures on $\boldsymbol{X} / Z$, with $\operatorname{dim}_{\mathbb{C}} Z=k$ and $\operatorname{vdim}_{\mathbb{C}} \boldsymbol{X}=n+k$. Write $X_{\text {an }}, Z_{\text {an }}$ for the sets of $\mathbb{C}$-points of $X=t_{0}(\boldsymbol{X}), Z$ with the complex analytic topology, and suppose $X_{\mathrm{an}}$ is paracompact. Then we can construct a relative Kuranishi atlas $\mathcal{K}, \varpi_{J: J \in A}$ for $\pi_{\mathrm{an}}: X_{\mathrm{an}} \rightarrow Z_{\mathrm{an}}$ of real dimension $n+2 k$, as in Definition 2.15, with $\varpi_{J}: U_{J} \rightarrow Z_{\text {an }}$ a submersion. If $\boldsymbol{X}$ is quasicompact (equivalently, of finite type) then we can take $\mathcal{K}$ to be finite.

Proof. First choose a family $\left\{\left(A_{i}^{\bullet}, \boldsymbol{\alpha}_{i}, \beta_{i}\right): i \in I\right\}$, where $A_{i}^{\bullet} \in \mathbf{c d g a}_{\mathbb{C}}$ is a standard form cdga, and $\boldsymbol{\alpha}_{i}$ : $\mathbf{S p e c} A_{i}^{\bullet} \hookrightarrow \boldsymbol{X}$ is a Zariski open inclusion in $\mathbf{d S c h}_{\mathbb{C}}$ for each $i$ in $I$, an indexing set, and $\beta_{i}: B \rightarrow A_{i}^{0}$ is a smooth morphism of classical $\mathbb{C}$-algebras such that (3.1) homotopy commutes, with $\left\{R_{i}:=\left(\operatorname{Im} \boldsymbol{\alpha}_{i}\right)_{\text {an }}: i \in I\right\}$ an open cover of the complex analytic topological space $X_{\mathrm{an}}$. This is possible by a relative version of Theorem 2.5 , easily proved by modifying the proof of [4, Th. 4.1] to work over the base $Z=\operatorname{Spec} B$. Apply Theorem 3.1 to get data $A_{J}^{\bullet} \in \mathbf{c d g a}_{\mathbb{C}}, \boldsymbol{\alpha}_{J}: \operatorname{Spec} A_{J}^{\bullet} \hookrightarrow \boldsymbol{X}, \beta_{J}: B \rightarrow A_{J}^{0}$ for finite $\emptyset \neq J \subseteq I$ and quasi-free morphisms $\Phi_{J K}: A_{K}^{\bullet} \rightarrow A_{J}^{\bullet}$, for all finite $\emptyset \neq K \subseteq J \subseteq I$. 
Use the notation of $₫ 3.2$ to rewrite $A_{J}^{\bullet}, \beta_{J}, \Phi_{J K}$ in terms of complex geometry. As in Corollary 3.5 this gives data $V_{J}, \tau_{J}, E_{J}, F_{J}, s_{J}, t_{J}, \psi_{J}, R_{J}$ for all finite $\emptyset \neq J \subseteq I$, and $\phi_{J K}, \chi_{J K}, \xi_{J K}$ for all finite $\emptyset \neq K \subseteq J \subseteq I$. Note that the holomorphic submersions $\tau_{J}: V_{J} \rightarrow Z_{\text {an }}$ with $\tau_{J}=\tau_{K} \circ \phi_{J K}$ for $K \subseteq J$ were not used in $\sqrt[3.3]{3.6}$ as there $Z_{\text {an }}$ was the point $*$, but now we need them.

Proposition 3.14 now also holds in our relative situation. Its proof in 66.2 uses Theorem 3.7 and Lemma 3.12, which as above hold in the relative situation with Definition 3.21 and $T\left(V_{J} / Z_{\text {an }}\right)$ in place of Definition 3.6 and $T V_{J}$. As in the proof of Theorem 3.15 we have now constructed a Kuranishi atlas $\mathcal{K}$ on $X_{\text {an }}$, with dimension $n+2 k$. Setting $\varpi_{J}:=\left.\tau_{J}\right|_{U_{J}}: U_{J} \rightarrow Z_{\text {an }}$ for $J \in A$, we see that $\mathcal{K}, \varpi_{J: J \in A}$ is a relative Kuranishi atlas for $\pi_{\text {an }}$, with $\varpi_{J}$ a submersion. If $X$ is quasicompact we can take $I$ finite, so $A$ and $\mathcal{K}$ are finite.

We then deduce the following relative analogue of Theorem 3.16.

Theorem 3.24. (i) Let $\boldsymbol{X}$ be a separated derived $\mathbb{C}$-scheme, $Z=\operatorname{Spec} B$ a smooth, connected, classical affine $\mathbb{C}$-scheme, $\boldsymbol{\pi}: \boldsymbol{X} \rightarrow Z$ a morphism, and $\left[\omega_{\boldsymbol{X} / Z}\right]$ a family of -2 -shifted symplectic structures on $\boldsymbol{X} / Z$, with $\operatorname{dim}_{\mathbb{C}} Z=k$ and $\operatorname{vdim}_{\mathbb{C}} \boldsymbol{X}=n+k$. Write $X_{\mathrm{an}}, Z_{\mathrm{an}}$ for the sets of $\mathbb{C}$-points of $X=t_{0}(\boldsymbol{X}), Z$ with the complex analytic topology, and suppose $X_{\mathrm{an}}$ is second countable.

Then we can make the topological space $X_{\mathrm{an}}$ into a derived manifold $\boldsymbol{X}_{\mathrm{dm}}$ with real virtual dimension $\operatorname{vdim}_{\mathbb{R}} \boldsymbol{X}_{\mathrm{dm}}=n+2 k$, in any of the senses (a) Joyce's m-Kuranishi spaces mKur [22, §4.7], (b) Joyce's d-manifolds dMan [18, 20,

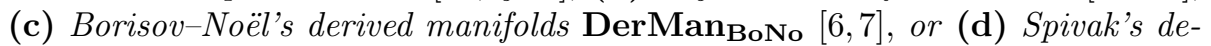

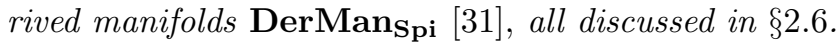

(ii) We can also define a morphism of derived manifolds $\boldsymbol{\pi}_{\mathrm{dm}}: \boldsymbol{X}_{\mathrm{dm}} \rightarrow Z_{\mathrm{an}}$, with underlying continuous map $\pi_{\mathrm{an}}: X_{\mathrm{an}} \rightarrow Z_{\mathrm{an}}$.

(iii) For each $z \in Z_{\mathrm{an}}$, the fibre $\boldsymbol{X}_{\mathrm{dm}}^{z}=\boldsymbol{\pi}_{\mathrm{dm}}^{-1}(z)=\boldsymbol{X}_{\mathrm{dm}} \times_{\boldsymbol{\pi}_{\mathrm{dm}}, Z_{\mathrm{an}}, z} *$ is a derived manifold with $\operatorname{vdim}_{\mathbb{R}} \boldsymbol{X}_{\mathrm{dm}}^{z}=n$. From Definition 3.20, $\boldsymbol{X}^{z}=\boldsymbol{\pi}^{-1}(z)$ has a -2 shifted symplectic structure $\omega_{\boldsymbol{X}^{z}}^{*}$, and both $\boldsymbol{X}_{\mathrm{dm}}^{z}, \boldsymbol{X}^{z}$ have (complex analytic) topological space $\pi_{\mathrm{an}}^{-1}(z) \subseteq X_{\mathrm{an}}$. Then $\boldsymbol{X}_{\mathrm{dm}}^{z}$ is up to equivalence a possible choice for the derived manifold associated to $\left(\boldsymbol{X}^{z}, \omega_{\boldsymbol{X}^{z}}^{*}\right)$ in Theorem 3.16.

Proof. Parts (i),(ii) follow from Theorems 2.18 and 3.23 For (iii), if $z \in$ $Z_{\text {an }}$ then as $\tau_{J}: V_{J} \rightarrow Z_{\text {an }}$ is a holomorphic submersion for $J \in A$, the fibre $V_{J}^{z}:=\tau_{J}^{-1}(z)$ is a complex submanifold of $V_{J}$. Setting $U_{J}^{z}=U_{J} \cap$ $V_{J}^{z}$ and writing $E_{J}^{z}, F_{J}^{z}, s_{J}^{z}, t_{J}^{z}$ for the restrictions of $E_{J}, F_{J}, s_{J}, t_{J}$ to $V_{J}^{z}$, and $E_{J}^{-z}, E_{J}^{+z}, s_{J}^{+z}, \psi_{J}^{+z}$ for the restrictions of $E_{J}^{-}, E_{J}^{+}, s_{J}^{+}, \psi_{J}^{+}$to $U_{J}^{z}$, we see that $I, A, V_{J}^{z}, E_{J}^{z}, F_{J}^{z}, s_{J}^{z}, t_{J}^{z}, U_{J}^{z}, \ldots$ are a possible choice for the data $I, A, V_{J}, E_{J}, \ldots$ in the application of Theorems 3.15 and 3.16 to $\left(\boldsymbol{X}^{z}, \omega_{\boldsymbol{X}^{z}}^{*}\right)$. But from facts about fibre products of derived manifolds in [18, 20, 23, we see that the derived manifold $\boldsymbol{X}_{\mathrm{dm}}^{z}=\boldsymbol{X}_{\mathrm{dm}} \times_{\boldsymbol{\pi}_{\mathrm{dm}}, Z_{\mathrm{an}}, z} *$ may be constructed as above from the data $I, A, U_{J}^{z}, E_{J}^{+z}, s_{J}^{+z}, \psi_{J}^{+z}, \ldots$. The theorem follows.

Next we discuss orientations, generalizing $\$ 2.4$ and 33.6 to the relative case. Here is the analogue of Definition 2.12 
Definition 3.25. Let $\boldsymbol{X}$ be a derived $\mathbb{C}$-scheme, $Z=\operatorname{Spec} B$ a smooth, connected, classical affine $\mathbb{C}$-scheme, $\boldsymbol{\pi}: \boldsymbol{X} \rightarrow Z$ a morphism, and $\left[\omega_{\boldsymbol{X} / Z}\right] \in$ $H^{-2}\left(\mathbb{L}_{\boldsymbol{X} / Z}\right)$ a family of -2 -shifted symplectic structures on $\boldsymbol{X} / Z$. Then as in (2.4), $\left[\omega_{\boldsymbol{X} / Z}\right]$ induces a canonical isomorphism of line bundles on $X=t_{0}(\boldsymbol{X})$ :

$$
\iota_{\boldsymbol{X} / Z, \omega_{\boldsymbol{X} / Z}}:\left[\operatorname{det}\left(\left.\mathbb{L}_{\boldsymbol{X} / Z}\right|_{X}\right)\right]^{\otimes^{2}} \longrightarrow \mathcal{O}_{X} \cong \mathcal{O}_{X}^{\otimes^{2}} .
$$

An orientation for $\left(\boldsymbol{\pi}: \boldsymbol{X} \rightarrow Z,\left[\omega_{\boldsymbol{X} / Z}\right]\right)$ is an isomorphism $o: \operatorname{det}\left(\left.\mathbb{L}_{\boldsymbol{X} / Z}\right|_{X}\right) \rightarrow$ $\mathcal{O}_{X}$ such that $o \otimes o=\iota_{\boldsymbol{X} / Z, \omega_{X / Z}}$.

Here is the relative analogue of Proposition 3.17 In (b),(c), we could use also use notions of relative orientation for $\left(X_{\mathrm{an}}, \mathcal{K}\right) \rightarrow Z_{\text {an }}$ and $\boldsymbol{X}_{\mathrm{dm}} \rightarrow Z_{\mathrm{an}}$. But as $Z_{\text {an }}$ is a complex manifold with a natural orientation, these are equivalent to absolute orientations for $\left(X_{\mathrm{an}}, \mathcal{K}\right), \boldsymbol{X}_{\mathrm{dm}}$, so we do not bother. The proof is an easy modification of that in 6.3 .

Proposition 3.26. In the situation of Theorems 3.23 and 3.24, there are canonical 1-1 correspondences between:

(a) Orientations on $\left(\boldsymbol{\pi}: \boldsymbol{X} \rightarrow Z,\left[\omega_{\boldsymbol{X} / Z}\right]\right)$ in the sense of Definition 3.25 ,

(b) Orientations on $\left(X_{\mathrm{an}}, \mathcal{K}\right)$ in the sense of 92.5 and

(c) Orientations on $\boldsymbol{X}_{\mathrm{dm}}$ in the sense of $\$ 2.6 .2$.

The relative analogue of Proposition 3.18 does hold, but we will not prove it, as we do not need it. The next theorem says that the virtual classes $\left[\boldsymbol{X}_{\mathrm{dm}}\right]_{\mathrm{dbo}},\left[\boldsymbol{X}_{\mathrm{dm}}\right]_{\text {virt }}$ of a proper oriented -2 -shifted symplectic derived $\mathbb{C}$-scheme $\left(\boldsymbol{X}, \omega_{\boldsymbol{X}}^{*}\right)$ defined in Corollary 3.19 are unchanged under deformation in families. Note that it is essential that the base $\mathbb{C}$-scheme $Z$ be connected in Theorem 3.27 .

Theorem 3.27. Let $\boldsymbol{X}$ be a separated derived $\mathbb{C}$-scheme, $Z=\operatorname{Spec} B$ a smooth, connected, classical affine $\mathbb{C}$-scheme, $\boldsymbol{\pi}: \boldsymbol{X} \rightarrow Z$ a proper morphism, and $\left[\omega_{\boldsymbol{X} / Z}\right]$ a family of -2 -shifted symplectic structures on $\boldsymbol{X} / Z$, equipped with an orientation, with $\operatorname{dim}_{\mathbb{C}} Z=k$ and $\operatorname{vdim}_{\mathbb{C}} \boldsymbol{X}=n+k$.

For each $z \in Z_{\text {an }}$ we have a proper, oriented -2 -shifted symplectic $\mathbb{C}$-scheme $\left(\boldsymbol{X}^{z}, \omega_{\boldsymbol{X}^{z}}^{*}\right)$ with $\operatorname{vdim} \boldsymbol{X}^{z}=n$, so Corollary 3.19 defines a d-bordism class $\left[\boldsymbol{X}_{\mathrm{dm}}^{z}\right]_{\mathrm{dbo}} \in d B_{n}(*)$ and a virtual class $\left[\boldsymbol{X}_{\mathrm{dm}}^{z}\right]_{\mathrm{virt}} \in H_{n}\left(X_{\mathrm{an}}^{z} ; \mathbb{Z}\right)$, depending only on $\left(\boldsymbol{X}^{z}, \omega_{\boldsymbol{X}^{z}}^{*}\right)$. Then $\left[\boldsymbol{X}_{\mathrm{dm}}^{z_{1}}\right]_{\mathrm{dbo}}=\left[\boldsymbol{X}_{\mathrm{dm}}^{z_{2}}\right]_{\mathrm{dbo}}$ and $\imath_{*}^{z_{1}}\left(\left[\boldsymbol{X}_{\mathrm{dm}}^{z_{1}}\right]_{\mathrm{virt}}\right)=i_{*}^{z_{2}}\left(\left[\boldsymbol{X}_{\mathrm{dm}}^{z_{2}}\right]_{\mathrm{virt}}\right)$ for all $z_{1}, z_{2} \in Z_{\mathrm{an}}$, where $\imath_{*}^{z}\left(\left[\boldsymbol{X}_{\mathrm{dm}}^{z}\right]_{\mathrm{virt}}\right) \in H_{n}\left(X_{\mathrm{an}} ; \mathbb{Z}\right)$ is the pushforward under the inclusion $\imath^{z}: X_{\mathrm{an}}^{z} \hookrightarrow X_{\mathrm{an}}$.

Proof. Theorem 3.24 constructs a derived manifold $\boldsymbol{X}_{\mathrm{dm}}$ with vdim $\boldsymbol{X}_{\mathrm{dm}}=$ $n+2 k$ and a morphism $\boldsymbol{\pi}_{\mathrm{dm}}: \boldsymbol{X}_{\mathrm{dm}} \rightarrow Z_{\mathrm{an}}$, which is proper as $\boldsymbol{\pi}$ is proper, and Proposition 3.26 gives an orientation on $\boldsymbol{X}_{\mathrm{dm}}$.

Let $z_{1}, z_{2} \in Z_{\text {an }}$. As $Z$ is connected we can choose a smooth map $\gamma:[0,1] \rightarrow$ $Z_{\text {an }}$ with $\gamma(0)=z_{1}$ and $\gamma(1)=z_{2}$. The fibre product

$$
\boldsymbol{W}_{\mathrm{dm}}=\boldsymbol{X}_{\mathrm{dm}} \times_{\boldsymbol{\pi}_{\mathrm{dm}}, Z_{\mathrm{an}, \gamma}}[0,1]
$$


exists as a derived manifold with boundary by [19, §7.5], [20, §7.6], [23], with $\operatorname{vdim} \boldsymbol{W}_{\mathrm{dm}}=n+1$, and $\boldsymbol{W}_{\mathrm{dm}}$ is compact as $[0,1]$ is and $\boldsymbol{\pi}_{\mathrm{dm}}$ is proper, and oriented since $\boldsymbol{X}_{\mathrm{dm}}, Z_{\mathrm{an}},[0,1]$ are. As $\partial \boldsymbol{X}_{\mathrm{dm}}=\partial Z_{\mathrm{an}}=\emptyset$, the boundary is

$$
\partial \boldsymbol{W}_{\mathrm{dm}}=\boldsymbol{X}_{\mathrm{dm}} \times_{\boldsymbol{\pi}_{\mathrm{dm}}, Z_{\mathrm{an}, \gamma}} \partial[0,1]=\boldsymbol{X}_{\mathrm{dm}}^{z_{1}} \amalg \boldsymbol{X}_{\mathrm{dm}}^{z_{2}},
$$

where $\boldsymbol{X}_{\mathrm{dm}}^{z_{1}}, \boldsymbol{X}_{\mathrm{dm}}^{z_{2}}$ are the fibres of $\boldsymbol{\pi}_{\mathrm{dm}}: \boldsymbol{X}_{\mathrm{dm}} \rightarrow Z_{\mathrm{an}}$ at $z_{1}, z_{2}$.

Since $\partial[0,1]=-\{0\} \amalg\{1\}$ in oriented 0-manifolds, we have $\partial \boldsymbol{W}_{\mathrm{dm}}=$ $-\boldsymbol{X}_{\mathrm{dm}}^{z_{1}} \amalg \boldsymbol{X}_{\mathrm{dm}}^{z_{2}}$ in oriented derived manifolds. Therefore Definition 2.20 gives $\left[\boldsymbol{X}_{\mathrm{dm}}^{z_{1}}\right]_{\mathrm{dbo}}=\left[\boldsymbol{X}_{\mathrm{dm}}^{z_{2}}\right]_{\mathrm{dbo}}$ in $d B_{n}(*)$. By Theorem $3.22(\mathrm{c}) \boldsymbol{X}_{\mathrm{dm}}^{z_{1}}, \boldsymbol{X}_{\mathrm{dm}}^{z_{2}}$ are outcomes of Theorem 3.16 applied to $\left(\boldsymbol{X}^{z_{1}}, \omega_{\boldsymbol{X}^{z_{1}}}^{*}\right),\left(\boldsymbol{X}^{z_{2}}, \omega_{\boldsymbol{X}^{z_{2}}}^{*}\right)$, so $\left[\boldsymbol{X}_{\mathrm{dm}}^{z_{1}}\right]_{\mathrm{dbo}},\left[\boldsymbol{X}_{\mathrm{dm}}^{z_{2}}\right]_{\mathrm{dbo}}$ are the d-bordism classes associated to $\left(\boldsymbol{X}^{z_{1}}, \omega_{\boldsymbol{X}^{z_{1}}}^{*}\right),\left(\boldsymbol{X}^{z_{2}}, \omega_{\boldsymbol{X}^{z_{2}}}^{*}\right)$ in Corollary 3.19. A similar argument works for the homology classes.

Remark 3.28. The assumptions that $Z$ is smooth, classical, and affine, and $\boldsymbol{X}$ is separated, in Theorem 3.27 are easily removed; we can work over a base $\boldsymbol{Z}$ which is a general classical or derived $\mathbb{C}$-scheme, provided it is connected.

To see this, suppose $\boldsymbol{\pi}: \boldsymbol{X} \rightarrow \boldsymbol{Z}$ is a proper morphism of derived $\mathbb{C}$-schemes with $\boldsymbol{Z}$ connected, and $\left[\omega_{\boldsymbol{X} / \boldsymbol{Z}}\right] \in H^{-2}\left(\mathbb{L}_{\boldsymbol{X} / \boldsymbol{Z}}\right)$ is a family of -2-shifted symplectic structures on $\boldsymbol{X} / \boldsymbol{Z}$ equipped with an orientation, extending Definitions 3.20 and 3.25 to general $\boldsymbol{Z}$ in the obvious way.

Suppose $z_{1}, z_{2} \in Z_{\text {an }}$. As $\boldsymbol{Z}$ is connected we can find a sequence $z_{1}=$ $z^{0}, z^{1}, \ldots, z^{N}=z_{2}$ of points in $Z_{\mathrm{an}}$, and a sequence of smooth, connected, affine curves $C^{1}, \ldots, C^{N}$ over $\mathbb{C}$ with morphisms $\pi^{i}: C^{i} \rightarrow \boldsymbol{Z}$, such that $\pi^{i}\left(C^{i}\right)$ contains $z^{i-1}, z^{i}$ for $i=1, \ldots, N$. Then $\boldsymbol{X}^{i}=\boldsymbol{X} \times_{\boldsymbol{\pi}, \boldsymbol{Z}, \pi^{i}}^{h} C^{i}$ is a derived $\mathbb{C}$-scheme, and $\left[\omega_{\boldsymbol{X} / \boldsymbol{Z}}\right]$ pulls back to a family $\left[\omega_{\boldsymbol{X}^{i} / C^{i}}\right]$ of oriented -2 -shifted symplectic structures on $\boldsymbol{X}^{i} / C^{i}$. Applying Theorem 3.27 to $\left(\boldsymbol{X}^{i} \rightarrow C^{i},\left[\omega_{\boldsymbol{X}^{i} / C^{i}}\right]\right)$ we see that $\left[\boldsymbol{X}_{\mathrm{dm}}^{z^{i-1}}\right]=\left[\boldsymbol{X}_{\mathrm{dm}}^{z^{i}}\right]$ in $d B_{n}(*)$ for $i=1, \ldots, N$, so that

$$
\left[\boldsymbol{X}_{\mathrm{dm}}^{z_{1}}\right]_{\mathrm{dbo}}=\left[\boldsymbol{X}_{\mathrm{dm}}^{z^{0}}\right]=\left[\boldsymbol{X}_{\mathrm{dm}}^{z^{1}}\right]=\cdots=\left[\boldsymbol{X}_{\mathrm{dm}}^{z^{N}}\right]=\left[\boldsymbol{X}_{\mathrm{dm}}^{z_{2}}\right]_{\mathrm{dbo}} .
$$

The same argument works for virtual classes $\left[\boldsymbol{X}_{\mathrm{dm}}^{z_{i}}\right]_{\mathrm{virt}}$ in homology.

We took $\boldsymbol{Z}$ to be smooth above to avoid defining families $\boldsymbol{\pi}_{\mathrm{dm}}: \boldsymbol{X}_{\mathrm{dm}} \rightarrow \boldsymbol{Z}$ of derived manifolds over a base $\boldsymbol{Z}$ which is not a (derived) manifold.

\section{8 'Holomorphic Donaldson invariants' of C-Y 4-folds}

We now outline how the results of 33.1 can be used to define new enumerative invariants of (semi)stable coherent sheaves on Calabi-Yau 4-folds $Y$, which we could call 'holomorphic Donaldson invariants', and which should be unchanged under deformations of $Y$. A related programme using gauge theory has recently been proposed by Cao and Leung [8 10, which we discuss in 33.9 .

We begin by discussing Donaldson-Thomas invariants $D T^{\alpha}(\tau)$ of CalabiYau 3-folds, introduced by Thomas 32. Suppose $Z$ is a Calabi-Yau 3-fold over $\mathbb{C}$ with an ample line bundle $\mathcal{O}_{Z}(1)$, which defines a Gieseker stability condition $\tau$ on coherent sheaves on $Z$, and $\alpha \in H^{\text {even }}(Z ; \mathbb{Q})$. Then one can form coarse 
moduli $\mathbb{C}$-schemes $\mathcal{M}_{\mathrm{st}}^{\alpha}(\tau), \mathcal{M}_{\mathrm{ss}}^{\alpha}(\tau)$ of $\tau$-(semi)stable coherent sheaves on $Z$ of Chern character $\alpha$, with $\mathcal{M}_{\mathrm{st}}^{\alpha}(\tau) \subseteq \mathcal{M}_{\mathrm{ss}}^{\alpha}(\tau)$ Zariski open, and $\mathcal{M}_{\mathrm{ss}}^{\alpha}(\tau)$ proper.

Thomas [32] showed that $\mathcal{M}_{\mathrm{st}}^{\alpha}(\tau)$ carries an 'obstruction theory' $\phi: E^{\bullet} \rightarrow$ $\mathbb{L}_{\mathcal{M}_{\mathrm{st}}^{\alpha}(\tau)}$ of virtual dimension 0, in the sense of Behrend and Fantechi [1]. Thus, if there are no strictly $\tau$-semistable sheaves in class $\alpha$, so that $\mathcal{M}_{\mathrm{st}}^{\alpha}(\tau)=\mathcal{M}_{\mathrm{ss}}^{\alpha}(\tau)$ and $\mathcal{M}_{\mathrm{st}}^{\alpha}(\tau)$ is proper, then [1] gives a virtual count $D T^{\alpha}(\tau)=\left[\mathcal{M}_{\mathrm{st}}^{\alpha}(\tau)\right]_{\mathrm{virt}} \in \mathbb{Z}$. Thomas proved that $D T^{\alpha}(\tau)$ is unchanged under continuous deformations of $Z$.

Later, Joyce and Song [24] extended the definition of $D T^{\alpha}(\tau)$ to invariants $\overline{D T^{\alpha}}(\tau) \in \mathbb{Q}$ for all $\alpha \in H^{\text {even }}(Z ; \mathbb{Q})$, dropping the condition that there are no strictly $\tau$-semistable sheaves in class $\alpha$, and proved a wall-crossing formula for $\overline{D T}{ }^{\alpha}(\tau)$ under change of stability condition $\tau$. At about the same time, Kontsevich and Soibelman 25] defined a motivic generalization of DonaldsonThomas invariants (assuming existence of 'orientation data' as in 82.4 ), and proved their own wall-crossing formula under change of $\tau$.

Thomas [32] called his invariants $D T^{\alpha}(\tau)$ 'holomorphic Casson invariants', though they are now generally known as Donaldson-Thomas invariants. Here Casson invariants are integer invariants of oriented real 3-manifolds $Z_{\mathbb{R}}$ which are homology 3-spheres, which 'count' flat connections on $Z_{\mathbb{R}}$.

This followed a programme of Donaldson and Thomas [13], which starting with some well-known geometry in real dimensions 2,3 and 4 , aimed to find analogues in complex dimensions 2,3 and 4; so the complex analogues of homology 3 -spheres, and flat connections upon them, are Calabi-Yau 3-folds, and holomorphic vector bundles (or coherent sheaves) upon them.

Donaldson invariants 12] are invariants of compact, oriented 4-manifolds $Y_{\mathbb{R}}$, defined by 'counting' moduli spaces $\mathcal{M}_{\text {inst }}^{\alpha}$ of $\mathrm{SU}(2)$-instantons $E$ on $Y_{\mathbb{R}}$ with $c_{2}(E)=\alpha \in \mathbb{Z}$. In contrast to Casson and Donaldson-Thomas invariants, the (virtual) dimension $d^{\alpha}$ of $\mathcal{M}_{\text {inst }}^{\alpha}$ need not be zero. Oversimplifying / lying a bit, one first constructs an orientation on $\mathcal{M}_{\text {inst }}^{\alpha},[12, \S 5.4]$. Then we have a virtual class $\left[\mathcal{M}_{\text {inst }}^{\alpha}\right]_{\text {virt }} \in H_{d^{\alpha}}\left(\mathcal{M}_{\text {inst }}^{\alpha} ; \mathbb{Z}\right)$. For each $\beta \in H_{2}\left(Y_{\mathbb{R}} ; \mathbb{Z}\right)$ we construct a natural cohomology class $\mu(\beta) \in H^{2}\left(\mathcal{M}_{\text {inst }}^{\alpha} ; \mathbb{Z}\right)$, with $\mu\left(\beta_{1}+\beta_{2}\right)=\mu\left(\beta_{1}\right)+\mu\left(\beta_{2}\right)$. Then if $d^{\alpha}=2 k$, we define Donaldson invariants $D^{\alpha}\left(\beta_{1}, \ldots, \beta_{k}\right)=\left(\mu\left(\beta_{1}\right) \cup \cdots \cup\right.$ $\left.\mu\left(\beta_{k}\right)\right) \cdot\left[\mathcal{M}_{\text {inst }}^{\alpha}\right]_{\text {virt }} \in \mathbb{Z}$ for all $\beta_{1}, \ldots, \beta_{k} \in H_{2}\left(Y_{\mathbb{R}} ; \mathbb{Z}\right)$. We can think of $D^{\alpha}$ as a $\mathbb{Z}$-valued homogeneous degree $k$ polynomial on $H_{2}\left(Y_{\mathbb{R}} ; \mathbb{Z}\right)$.

We propose, following [13, to define 'holomorphic Donaldson invariants' of Calabi-Yau 4-folds. The gauge theory ideas which were the primary focus of 13 will be discussed in $\$ 3.9$, here we work in the world of (derived) algebraic geometry. Suppose $Y$ is a Calabi-Yau 4 -fold over $\mathbb{C}$ (i.e. $Y$ is smooth and projective with $H^{i}\left(\mathcal{O}_{Y}\right)=\mathbb{C}$ if $i=0,4$ and $H^{i}\left(\mathcal{O}_{Y}\right)=0$ otherwise), and $\alpha=\left(\alpha^{0}, \alpha^{2}, \alpha^{4}, \alpha^{6}, \alpha^{8}\right) \in H^{\text {even }}(Y ; \mathbb{Q})$. As above we can form coarse moduli $\mathbb{C}$-schemes $\mathcal{M}_{\mathrm{st}}^{\alpha}(\tau) \subseteq \mathcal{M}_{\mathrm{ss}}^{\alpha}(\tau)$ of Gieseker (semi)stable coherent sheaves on $Y$ of Chern character $\alpha$, with $\mathcal{M}_{\mathrm{ss}}^{\alpha}(\tau)$ proper.

To make contact with the work of 33.1 we need to show:

Claim 3.29. There is a-2-shifted symplectic derived $\mathbb{C}$-scheme $\left(\mathcal{M}_{\mathrm{st}}^{\alpha}(\tau), \omega^{*}\right)$, natural up to equivalence, with classical truncation $t_{0}\left(\mathcal{M}_{\mathrm{st}}^{\alpha}(\tau)\right)=\mathcal{M}_{\mathrm{st}}^{\alpha}(\tau)$, of virtual dimension $\operatorname{vdim}_{\mathbb{C}} \mathcal{M}_{\mathrm{st}}^{\alpha}(\tau)=d^{\alpha}:=2-\operatorname{deg}(\alpha \cup \bar{\alpha} \cup \operatorname{td}(T Y))_{8}$, where 
$\bar{\alpha}=\left(\alpha^{0},-\alpha^{2}, \alpha^{4},-\alpha^{6}, \alpha^{8}\right)$, and $\operatorname{td}(-)$ is the Todd class.

Pantev et al. [30, §2.1] prove the analogue of Claim 3.29] in the context of (derived) Artin stacks, but we want to reduce to (derived) schemes. Roughly this means factoring out the $\mathbb{C}^{*}$ stabilizer groups at each point of the $\tau$-stable derived moduli stack. Actually, it should not be difficult to extend 3.1 to derived algebraic $\mathbb{C}$-spaces rather than derived $\mathbb{C}$-schemes, and then it would be enough to construct $\mathcal{M}_{\text {st }}^{\alpha}(\tau)$ as a derived algebraic $\mathbb{C}$-space.

Next we would need to answer the:

Question 3.30. Does $\left(\mathcal{M}_{\mathrm{st}}^{\alpha}(\tau), \omega^{*}\right)$ in Claim 3.29 have a natural orientation, in the sense of 2.4 possibly depending on some choice of data on $Y$ ?

Following the argument of Donaldson [12, §5.4], Cao and Leung [10, Th. 2.2] prove an orientability result, which should translate to the statement that if the Calabi-Yau 4-fold $Y$ has holonomy SU(4) with $H_{*}(Y ; \mathbb{Z})$ torsion-free, and $\mathcal{M}_{\text {st }}^{\alpha}(\tau)$ is a derived moduli scheme of coherent sheaves on $Y$, then orientations on $\mathcal{M}_{\text {st }}^{\alpha}(\tau)$ exist, though they do not construct a natural choice.

If both these problems are solved, then Theorem 3.16 makes $\mathcal{M}_{\mathrm{st}}^{\alpha}(\tau)$ an into a derived manifold $\mathcal{M}_{\mathrm{st}}^{\alpha}(\tau)_{\mathrm{dm}}$ of real virtual dimension $d^{\alpha}$, which is oriented by Proposition 3.17. If there are no strictly $\tau$-semistable sheaves in class $\alpha$ then $\mathcal{M}_{\mathrm{st}}^{\alpha}(\tau)_{\mathrm{dm}}$ is also compact, and has a d-bordism class $\left[\mathcal{M}_{\mathrm{st}}^{\alpha}(\tau)_{\mathrm{dm}}\right]_{\mathrm{dbo}}$ in $d B_{d^{\alpha}}(*)$ and virtual class $\left[\mathcal{M}_{\mathrm{st}}^{\alpha}(\tau)_{\mathrm{dm}}\right]_{\text {virt }}$ in $H_{d^{\alpha}}\left(\mathcal{M}_{\mathrm{st}}^{\alpha}(\tau)_{\mathrm{an}} ; \mathbb{Z}\right)$.

If $d^{\alpha}=0$ then $\left[\mathcal{M}_{\mathrm{st}}^{\alpha}(\tau)_{\mathrm{dm}}\right]_{\mathrm{dbo}} \in d B_{0}(*) \cong \mathbb{Z}$ is the virtual count we want. But if $d^{\alpha}>0$ we should aim to find suitable cohomology classes on $\mathcal{M}_{\mathrm{st}}^{\alpha}(\tau)$ an and integrate them over $\left[\mathcal{M}_{\mathrm{st}}^{\alpha}(\tau)_{\mathrm{dm}}\right]_{\text {virt }}$, as for Donaldson invariants above.

Claim 3.31. One can define natural cohomology classes $\mu(\beta)$ on $\mathcal{M}_{\mathrm{st}}^{\alpha}(\tau)$ an depending on homology classes $\beta$ on $Y$, which can be combined with $\left[\mathcal{M}_{\mathrm{st}}^{\alpha}(\tau)_{\mathrm{dm}}\right]_{\text {virt }}$ to give integer invariants, in a similar way to Donaldson invariants.

If $\mathcal{M}_{\mathrm{st}}^{\alpha}(\tau)$ is a fine moduli space, there is a universal sheaf $\mathcal{E}$ on $\mathcal{M}_{\mathrm{st}}^{\alpha}(\tau) \times Y$, with Chern classes $c_{i}(\mathcal{E}) \in H^{2 i}\left(\mathcal{M}_{\mathrm{st}}^{\alpha}(\tau)_{\text {an }} \times Y ; \mathbb{Q}\right) \cong \bigoplus_{k} H^{2 i-k}\left(\mathcal{M}_{\mathrm{st}}^{\alpha}(\tau)_{\text {an }} ; \mathbb{Q}\right) \otimes$ $H^{k}(Y ; \mathbb{Q})$, and we can make $\mu_{i}(\beta) \in H^{2 i-k}\left(\mathcal{M}_{\mathrm{st}}^{\alpha}(\tau)\right.$ an $\left.; \mathbb{Q}\right)$ by contracting $c_{i}(\mathcal{E})$ with $\beta \in H_{k}(Y ; \mathbb{Q})$. Using the results of $\$ 3.7$ we should be able to prove that the resulting invariants are unchanged under continuous deformations of $Y$.

This would take us to the same point as Thomas 32] in the Calabi-Yau 3fold case: we could 'count' moduli spaces $\mathcal{M}_{\mathrm{st}}^{\alpha}(\tau)$ for those classes $\alpha$ containing no strictly $\tau$-semistable sheaves, and get a deformation-invariant answer. Many questions would remain, for instance, how to count strictly $\tau$-semistables, wallcrossing formulae as in [24,25, computation in examples, and so on.

We hope to return to these issues in future work.

\subsection{Motivation from gauge theory, and ' $\mathrm{SU}(4)$ instantons'}

Finally we discuss some ideas of Donaldson and Thomas [13], which were part of the motivation for this paper, and the work of Cao and Leung [8] 10]. 
Let $Y$ be a Calabi-Yau 4-fold over $\mathbb{C}$, regarded as a compact real 8-manifold $Y$ with complex structure $J$, Ricci-flat Kähler metric $g$, Kähler form $\omega$, and holomorphic volume form $\Omega$. Fix a complex vector bundle $E \rightarrow Y$ of rank $r>0$ with Hermitian metric $h$ and Chern character $\operatorname{ch}(E)=\alpha$, and as in 8, 9, assume for simplicity that $c_{1}(E)=0$. Consider connections $\nabla$ on $E$ preserving $h$ with curvature $F \in C^{\infty}\left(\operatorname{End}(E) \otimes_{\mathbb{C}}\left(\Lambda^{2} T^{*} Y \otimes_{\mathbb{R}} \mathbb{C}\right)\right)$. The splitting

$$
\Lambda^{2} T^{*} Y \otimes_{\mathbb{R}} \mathbb{C}=\langle\omega\rangle_{\mathbb{C}} \oplus \Lambda_{0}^{1,1} T^{*} Y \oplus \Lambda^{2,0} T^{*} Y \oplus \Lambda^{0,2} T^{*} Y
$$

induces a corresponding decomposition $F=F^{\omega} \oplus F_{0}^{1,1} \oplus F^{2,0} \oplus F^{0,2}$.

We call $\nabla$ a Hermitian-Einstein connection if $F^{\omega}=F^{2,0}=F^{0,2}=0$. We can split $\nabla=\partial_{E} \oplus \bar{\partial}_{E}$, where $\bar{\partial}_{E}$ gives $E$ the structure of a holomorphic vector bundle on $(Y, J)$, as $F^{0,2}=0$. The Hitchin-Kobayashi correspondence says that if $\left(E, \bar{\partial}_{E}\right)$ is a holomorphic vector bundle and is slope-stable, then $\bar{\partial}_{E}$ extends to a unique Hermitian-Einstein connection $\nabla=\partial_{E} \oplus \bar{\partial}_{E}$ preserving $h$. Also, holomorphic vector bundles on $Y$ are algebraic. Thus, studying moduli spaces $\mathcal{M}_{\text {alg-vb }}^{\alpha}$ of stable algebraic vector bundles is roughly equivalent to studying moduli spaces $\mathcal{M}_{\mathrm{HE}}^{\alpha}$ of Hermitian-Einstein connections, modulo gauge.

As a system of p.d.e.s, the Hermitian-Einstein equations are overdetermined: there are $8 r^{2}$ unknowns, $13 r^{2}$ equations and $r^{2}$ gauge equivalences, with $8 r^{2}-$ $13 r^{2}-r^{2}<0$. Algebraically, this corresponds to the fact that the natural obstruction theory on $\mathcal{M}_{\text {alg-vb }}$ is not perfect, so we cannot form virtual classes.

Using $\Omega, g$ we can define real splittings $\Lambda^{2,0} T^{*} Y=\Lambda_{+}^{2,0} T^{*} Y \oplus \Lambda_{-}^{2,0} T^{*} Y$, $\Lambda^{0,2} T^{*} Y=\Lambda_{+}^{0,2} T^{*} Y \oplus \Lambda_{-}^{0,2} T^{*} Y$ and corresponding decompositions $F^{2,0}=F_{+}^{2,0} \oplus$ $F_{-}^{2,0}, F^{0,2}=F_{+}^{0,2} \oplus F_{-}^{0,2}$. Following Donaldson and Thomas [13, §3], we call $\nabla$ an $\mathrm{SU}(4)$-instanton if $F^{\omega}=F_{+}^{2,0}=F_{+}^{0,2}=0$. This gives $8 r^{2}$ unknowns, $7 r^{2}$ equations and $r^{2}$ gauge equivalences, with $8 r^{2}-7 r^{2}-r^{2}=0$. It is a determined elliptic system, so that we can hope to define virtual classes. This is special to Calabi-Yau 4-folds, a complex analogue of instantons on real 4-manifolds.

Writing $\mathcal{M}_{\mathrm{SU}(4)}^{\alpha}$ for the moduli space of SU(4)-instantons, we have $\mathcal{M}_{\mathrm{HE}}^{\alpha} \subseteq$ $\mathcal{M}_{\mathrm{SU}(4)}^{\alpha}$, as the $\mathrm{SU}(4)$ instanton equations are weaker than the HermitianEinstein equations. Now $\alpha=\operatorname{ch}(E) \in \bigoplus_{p=0}^{4} H^{p, p}(Y)$ if $E$ admits HermitianEinstein connections. Conversely, as in [13, p. 36], if $\alpha \in \bigoplus_{p} H^{p, p}(Y)$ then one can use $L^{2}$-norms of components of $F$ to show that any SU(4)-instanton is Hermitian-Einstein. Thus, either $\mathcal{M}_{\mathrm{HE}}^{\alpha}=\mathcal{M}_{\mathrm{SU}(4)}^{\alpha}$, or $\mathcal{M}_{\mathrm{HE}}^{\alpha}=\emptyset$.

However, the equality $\mathcal{M}_{\mathrm{HE}}^{\alpha}=\mathcal{M}_{\mathrm{SU}(4)}^{\alpha}$ holds only at the level of sets, or topological spaces. Since $\mathcal{M}_{\mathrm{HE}}^{\alpha}$ is defined by more equations, if we regard $\mathcal{M}_{\mathrm{HE}}^{\alpha}, \mathcal{M}_{\mathrm{SU}(4)}^{\alpha}$ as (derived) $C^{\infty}$-schemes, for instance, then $\mathcal{M}_{\mathrm{HE}}^{\alpha} \subsetneq \mathcal{M}_{\mathrm{SU}(4)}^{\alpha}$.

In the setting of $3.1-3.6$, we should compare $\mathcal{M}_{\mathrm{HE}}^{\alpha}$ (a Calabi-Yau 4-fold moduli space, without a virtual class, equivalent to an algebraic moduli scheme $\left.\mathcal{M}_{\text {alg-vb }}^{\alpha}\right)$ with the -2 -shifted symplectic derived $\mathbb{C}$-scheme $\left(\boldsymbol{X}, \omega_{\boldsymbol{X}}^{*}\right)$, and $\mathcal{M}_{\mathrm{SU}(4)}^{\alpha}$ (an elliptic moduli space, hopefully with a virtual class, equal to $\mathcal{M}_{\mathrm{HE}}^{\alpha}$ on the level of topological spaces) with the derived manifold $\boldsymbol{X}_{\mathrm{dm}}$. It was these ideas from Donaldson and Thomas 13 that led the authors to believe that one could modify a -2 -shifted symplectic derived $\mathbb{C}$-scheme to get a derived manifold with the same topological space, and so define a virtual class. 
Donaldson and Thomas [13] envisaged using gauge theory to define invariants of Calabi-Yau 4-folds 'counting' moduli spaces $\mathcal{M}_{\mathrm{SU}(4)}^{\alpha}$, and also invariants of compact Spin(7)-manifolds 'counting' moduli spaces of 'Spin(7)-instantons'.

This would require finding suitable compactifications $\overline{\mathcal{M}}_{\mathrm{SU}(4)}^{\alpha}$ of the moduli spaces $\mathcal{M}_{\mathrm{SU}(4)}^{\alpha}$, and giving them a nice enough geometric structure to define virtual classes, which is a formidably difficult problem in gauge theory in dimensions $>4$. A huge advantage of our approach is that, working in algebraic geometry, with moduli spaces of coherent sheaves rather than vector bundles, we often get compactness of moduli spaces for free, without doing any work.

Cao and Leung 8 10, also aim to define enumerative invariants of CalabiYau 4-folds $Y$, which they call ' $D T_{4}$-invariants', and their ideas overlap with ours. As for our outline in 3.8 , their general theory is still rather incomplete, but they prove many partial results, and do computations in examples.

Given a vector bundle moduli space $\mathcal{M}_{\text {alg-vb }}^{\alpha} \cong \mathcal{M}_{\mathrm{HE}}^{\alpha} \cong \mathcal{M}_{\mathrm{SU}(4)}^{\alpha}$ in topological spaces, assuming it is compact, and with an orientation (compare Question [3.30), Cao and Leung [9, §5] define a virtual class $\left[\mathcal{M}_{\mathrm{SU}(4)}^{\alpha}\right]_{\text {virt }}$ for $\mathcal{M}_{\mathrm{SU}(4)}^{\alpha}$, and contract this with some cohomology classes $\mu(\beta)$ (compare Claim 3.31) to get integer invariants, which they prove are unchanged under deformations of $Y$. All this involves fairly standard material from gauge theory.

They also discuss the case in which one has a compact moduli space of coherent sheaves $\mathcal{M}_{\text {coh-sh }}^{\alpha}$, which contains the vector bundle moduli space $\mathcal{M}_{\text {alg-vb }}^{\alpha}$ as an open subset. They want to define a virtual class for $\mathcal{M}_{\text {coh-sh }}^{\alpha}$, as we want to, and they can do this under the assumptions that either $\mathcal{M}_{\text {coh-sh }}^{\alpha}$ is smooth, or (in our language) that the -2 -shifted symplectic derived scheme $\left(\mathcal{M}_{\text {coh-sh }}^{\alpha}, \omega^{*}\right)$ is locally of the form $T^{*} \boldsymbol{X}[2]$ for $\boldsymbol{X}$ a quasi-smooth derived $\mathbb{C}$-scheme.

To compare our work with theirs: given $\mathcal{M}_{\text {alg-vb }}^{\alpha} \subset \mathcal{M}_{\text {coh-sh }}^{\alpha}$ as above, assuming Claim 3.29, our Theorem 3.16 gives $\mathcal{M}_{\text {coh-sh }}^{\alpha}$ the structure of a derived manifold, but one depending on arbitrary choices. By topologically identifying $\mathcal{M}_{\text {alg-vb }}^{\alpha} \cong \mathcal{M}_{\mathrm{SU}(4)}^{\alpha}$, in effect Cao and Leung make $\mathcal{M}_{\text {alg-vb }}^{\alpha}$ into a derived manifold, canonically up to equivalence (though depending on the Kähler metric $g$ and holomorphic volume form $\Omega$ ). However, there seems no reason why their derived manifold structure on $\mathcal{M}_{\text {alg-vb }}^{\alpha} \subset \mathcal{M}_{\text {coh-sh }}^{\alpha}$ should extend smoothly to $\mathcal{M}_{\text {coh-sh }}^{\alpha}$. This is a reason why our approach may in the end be more effective.

\section{Proof of Theorem 3.1}

In this proof we write $\mathbf{c d g a}_{\mathbb{C}}$ for the ordinary category of cdgas over $\mathbb{C}$, and cdga $_{\mathbb{C}}^{\infty}$ for the $\infty$-category of cdgas over $\mathbb{C}$, defined using the model structure on cdga $_{\mathbb{C}}$. All objects in cdga $\mathbb{C}_{\mathbb{C}}$ are fibrant. A cdga $A$ is cofibrant if it is a retract of a cdga $A^{\prime}$ which is almost-free, that is, free as a graded commutative algebra. If $\phi: A \rightarrow B$ is a morphism in cdga $\mathbb{C}_{\mathbb{C}}$ then $\phi: A \rightarrow B$ is also a morphism in $\mathbf{c d g a}_{\mathbb{C}}^{\infty}$. However, morphisms $\phi: A \rightarrow B$ in cdga $_{\mathbb{C}}^{\infty}$ may not correspond to morphisms $A \rightarrow B$ in cdga $\mathbb{C}_{\mathbb{C}}$ unless $A$ is cofibrant.

The spectrum functor Spec maps $\left(\mathbf{c d g a}_{\mathbb{C}}\right)^{\mathbf{o p}} \rightarrow \mathbf{d S c h}_{\mathbb{C}}$ and $\left(\mathbf{c d g a}_{\mathbb{C}}^{\infty}\right)^{\mathbf{o p}} \rightarrow$ $\mathbf{d S c h}_{\mathbb{C}}$, and $\left(\mathbf{c d g a}_{\mathbb{C}}^{\infty}\right)^{\mathbf{o p}} \rightarrow \mathbf{d S c h} \mathbf{S}_{\mathbb{C}}$ is an equivalence with the full $\infty$-subcategory 
of $\mathbf{d S c h}_{\mathbb{C}}$ with affine objects. So, morphisms $\phi: A \rightarrow B$ in $\mathbf{c d g a}_{\mathbb{C}}^{\infty}$ are essentially the same thing as morphisms $\mathbf{S p e c} B \rightarrow \mathbf{S p e c} A$ in $\mathbf{d S c h}_{\mathbb{C}}$.

Let $\boldsymbol{\pi}: \boldsymbol{X} \rightarrow Z=\operatorname{Spec} B$ and $\left\{\left(A_{i}^{\bullet}, \boldsymbol{\alpha}_{i}, \beta_{i}\right): i \in I\right\}$ be as in Theorem 3.1. Our task is to construct a standard form cdga $A_{J}^{\bullet}=\left(A_{J}^{*}, \mathrm{~d}\right)$, a Zariski open inclusion $\boldsymbol{\alpha}_{J}: \operatorname{Spec} A_{J}^{\bullet} \hookrightarrow \boldsymbol{X}$, and a morphism $\beta_{J}: B \rightarrow A_{J}^{0}$ for all finite $\emptyset \neq J \subseteq I$, and a quasi-free morphism $\Phi_{J K}: A_{K}^{\bullet} \rightarrow A_{J}^{\bullet}$ for all finite $\emptyset \neq K \subseteq J \subseteq I$, satisfying conditions. We will do this by induction on increasing $k=|J|$. Here is our inductive hypothesis:

Hypothesis 4.1. Let $k=1,2, \ldots$ be given. Then

(a) We are given finite subsets $S_{J}^{n}$ for all $\emptyset \neq J \subseteq I$ with $|J| \leqslant k$ and all $n=-1,-2, \ldots$.

(b) For all $\emptyset \neq J \subseteq I$ with $|J| \leqslant k$ we have $A_{J}^{0}=\bigotimes_{i \in J}^{\text {over } B} A_{i}^{0}$ as a smooth $\mathbb{C}$-algebra of pure dimension, where the tensor products are over $B$ using $\beta_{i}: B \rightarrow A_{i}^{0}$ to make $A_{i}^{0}$ into a $B$-algebra, so that if $J=\left\{i_{1}, \ldots, i_{j}\right\}$ then

$$
A_{J}^{0}=A_{i_{1}} \otimes_{B} A_{i_{2}} \otimes_{B} \cdots \otimes_{B} A_{i_{j}} .
$$

The morphism $\beta_{J}: B \rightarrow A_{J}^{0}$ is induced by (4.1) and the $\beta_{i}: B \rightarrow A_{i}^{0}$ for $i \in J$, and is smooth as the $\beta_{i}$ are.

(c) For all $\emptyset \neq J \subseteq I$ with $|J| \leqslant k$, as a graded $\mathbb{C}$-algebra, $A_{J}^{*}$ is freely generated over $A_{J}^{0}$ by generators $\bigsqcup_{\emptyset \neq K \subseteq J} S_{K}^{n}$ in degree $n$ for $n=-1,-2, \ldots$.

(d) For all $\emptyset \neq K \subseteq J \subseteq I$ with $|J| \leqslant k$, the morphism $\Phi_{J K}^{0}: A_{K}^{0} \rightarrow A_{J}^{0}$ in degree 0 is the morphism

$$
A_{K}^{0}=\bigotimes_{i \in K}^{\text {over } B} A_{i}^{0}=\left(\bigotimes_{i \in K}^{\text {over } B} A_{i}^{0}\right) \otimes_{B}\left(\bigotimes_{i \in J \backslash K}^{\text {over } B} B\right) \longrightarrow \bigotimes_{i \in J}^{\text {over } B} A_{i}^{0}=A_{J}^{0}
$$

induced by the morphisms id $: A_{i}^{0} \rightarrow A_{i}^{0}$ for $i \in K$ and $\beta_{i}: B \rightarrow A_{i}^{0}$ for $i \in J \backslash K$. Then $\Phi_{J K}: A_{K}^{*} \rightarrow A_{J}^{*}$ is the unique morphism of graded $\mathbb{C}$-algebras acting by $\Phi_{J K}^{0}$ in degree zero, and mapping $\Phi_{J K}: \gamma \mapsto \gamma$ for each $\gamma \in S_{L}^{n}$ for $\emptyset \neq L \subseteq K \subseteq J \subseteq I$ and $n=-1,-2, \ldots$, so that $\gamma$ is a free generator of both $A_{K}^{*}$ over $A_{K}^{0}$ and $A_{J}^{*}$ over $A_{J}^{0}$.

Note that $\Phi_{J K}^{0}: A_{K}^{0} \rightarrow A_{J}^{0}$ is a smooth morphism of $\mathbb{C}$-algebras of pure relative dimension, since id : $A_{i}^{0} \rightarrow A_{i}^{0}$ and $\beta_{i}: B \rightarrow A_{i}^{0}$ are. Also $\Phi_{J K}$ maps independent generators $\coprod_{\emptyset \neq L \subseteq K} S_{L}^{n}$ of $A_{K}^{*}$ over $A_{K}^{0}$ to independent generators of $A_{J}^{*}$ over $A_{J}^{0}$. Hence $\Phi_{J K}: A_{K}^{*} \rightarrow A_{J}^{*}$ is quasi-free.

Clearly $\beta_{J}=\Phi_{J K}^{0} \circ \beta_{K}=\Phi_{J K} \circ \beta_{K}: B \rightarrow A_{J}^{0}$.

Also, if $\emptyset \neq L \subseteq K \subseteq J \subseteq I$ with $|J| \leqslant K$ then clearly $\Phi_{J L}^{0}=\Phi_{J K}^{0} \circ \Phi_{K L}^{0}$ : $A_{L}^{0} \rightarrow A_{J}^{0}$, and $\Phi_{J L}=\Phi_{J K} \circ \Phi_{K L}: A_{L}^{*} \rightarrow A_{J}^{*}$.

(e) For all $\emptyset \neq J \subseteq I$ with $|J| \leqslant k$ and all $n=-1,-2, \ldots$, we are given maps $\delta_{J}^{n}: S_{J}^{n} \rightarrow A_{J}^{n+1}$.

(f) Let $\emptyset \neq J \subseteq I$ with $|J| \leqslant k$. Define $\mathrm{d}: A_{J}^{*} \rightarrow A_{J}^{*+1}$ uniquely by the conditions that $\mathrm{d}$ satisfies the Leibnitz rule, and

$$
\mathrm{d} \gamma=\Phi_{J K} \circ \delta_{K}^{n}(\gamma) \text { for all } \emptyset \neq K \subseteq J, n \leqslant-1 \text { and } \gamma \in S_{K}^{n} .
$$


We require that $\mathrm{d} \circ \mathrm{d}=0: A_{J}^{*} \rightarrow A_{J}^{*+2}$, so that $A_{J}^{\bullet}=\left(A_{J}^{*}, \mathrm{~d}\right)$ is a cdga.

This defines $A_{J}^{\bullet}=\left(A_{J}^{*}, \mathrm{~d}\right)$, as a standard form cdga over $\mathbb{C}$. Observe that if $\emptyset \neq K \subseteq J \subseteq I$ with $|J| \leqslant k$ then as $\Phi_{J K}: A_{K}^{*} \rightarrow A_{J}^{*}$ is a morphism of graded $\mathbb{C}$-algebras with $\Phi_{J K} \circ \mathrm{d} \gamma=\mathrm{d} \circ \Phi_{J K}(\gamma)$ for all $\gamma$ in the generating sets $\bigsqcup_{\emptyset \neq L \subseteq K} S_{L}^{n}$ for $A_{K}^{*}$ over $A_{K}^{0}$, we have $\Phi_{J K} \circ \mathrm{d}=\mathrm{d} \circ \Phi_{J K}: A_{K}^{*} \rightarrow$ $A_{J}^{*+1}$, and so $\Phi_{J K}: A_{K}^{\bullet} \rightarrow A_{J}^{\bullet}$ is a morphism of cdgas.

(g) For all $\emptyset \neq J \subseteq I$ with $|J| \leqslant k$, we are given a Zariski open inclusion $\boldsymbol{\alpha}_{J}: \operatorname{Spec} A_{J}^{\bullet} \hookrightarrow \boldsymbol{X}$, with image $\operatorname{Im} \boldsymbol{\alpha}_{J}=\bigcap_{i \in J} \operatorname{Im} \boldsymbol{\alpha}_{i}$, such that (3.2) homotopy commutes.

If $\emptyset \neq K \subseteq J \subseteq I$ with $|J| \leqslant k$ then (3.3) homotopy commutes.

Remark 4.2. (i) In Hypothesis 4.1, the only actual data required are the finite sets $S_{J}^{n}$ in (a), the maps $\delta_{J}^{n}: S_{J}^{n} \rightarrow A_{J}^{n+1}$ in (e), and the morphisms $\boldsymbol{\alpha}_{J}: \operatorname{Spec} A_{J}^{\bullet} \hookrightarrow \boldsymbol{X}$ in (g).

Also, the only statements requiring proof are that $\mathrm{d} \circ \mathrm{d}=0 \mathrm{in}(\mathrm{f})$, and that $\boldsymbol{\alpha}_{J}$ is a Zariski open inclusion with image $\bigcap_{i \in J} \operatorname{Im} \boldsymbol{\alpha}_{i}$, and that (3.2) and (3.3) homotopy commute in (g). All of (b),(c),(d) are definitions and deductions.

(ii) Most of the conclusions of Theorem 3.1 are immediate from the definitions in (a)-(g): that $A_{J}^{\bullet}$ is a standard form cdga, and $\beta_{J}: B \rightarrow A_{J}^{0}$ is smooth, and $\Phi_{J K}: A_{K}^{\bullet} \rightarrow A_{J}^{\bullet}$ is quasi-free, and $\beta_{J}=\Phi_{J K} \circ \beta_{K}$, and $\Phi_{J L}=\Phi_{J K} \circ \Phi_{K L}$.

For the first step in the induction, we prove Hypothesis 4.1 when $k=1$. Then the only subsets $\emptyset \neq J \subseteq I$ with $|J| \leqslant k$ are $J=\{i\}$ for $i \in I$, and the only subsets $\emptyset \neq K \subseteq J \subseteq I$ with $|J| \leqslant k$ are $J=K=\{i\}$ for $i \in I$.

As in Theorem 3.1 we are given data $\left\{\left(A_{i}^{\bullet}, \boldsymbol{\alpha}_{i}, \beta_{i}\right): i \in I\right\}$, where $A_{i}^{\bullet}$ is a standard form cdga, so that $A_{i}^{*}$ is freely generated over $A_{i}^{0}$ by finitely many generators in each degree $n=-1,-2, \ldots$, as in Definition 2.1. For each $i \in I$ and each $n=-1,-2, \ldots$, choose a subset $S_{\{i\}}^{n} \subseteq A_{i}^{n}$, as in part (a) for $J=\{i\}$, such that $A_{i}^{*}$ is freely generated over $A_{i}^{0}$ by $\coprod_{n \leqslant-1} S_{\{i\}}^{n}$. Set $A_{\{i\}}^{\bullet}=A_{i}^{\bullet}$ and $\beta_{\{i\}}=\beta_{i}$, so that parts (b),(c) hold for $J=\{i\}$.

Part (d) is a definition, and when $k=1$ only says that when $J=K=\{i\}$ we have $\Phi_{\{i\}\{i\}}=\mathrm{id}: A_{\{i\}}^{\bullet} \rightarrow A_{\{i\}}^{\bullet}$. For (e), define $\delta_{\{i\}}^{n}: S_{\{i\}}^{n} \rightarrow A_{\{i\}}^{n+1}=A_{i}^{n+1}$ by $\delta_{\{i\}}^{n}(\gamma)=\mathrm{d} \gamma$, using $\mathrm{d}$ in the cdga $A_{i}^{\bullet}=\left(A_{i}^{*}, \mathrm{~d}\right)$. Given (e), part (f) says that the differentials d in $A_{\{i\}}^{\bullet}=\left(A_{\{i\}}^{*}, \mathrm{~d}\right)$ and $A_{i}^{\bullet}=\left(A_{i}^{*}, \mathrm{~d}\right)$ agree, consistent with setting $A_{\{i\}}^{\bullet}=A_{i}^{\bullet}$, so d od $=0$ in $A_{\{i\}}^{\bullet}$ as $A_{i}^{\bullet}$ is a cdga.

For (g), if $i \in I$ define $\boldsymbol{\alpha}_{\{i\}}=\boldsymbol{\alpha}_{i}: A_{\{i\}}^{\bullet}=A_{i}^{\bullet} \rightarrow \boldsymbol{X}$. Then the assumptions on $\left\{\left(A_{i}^{\bullet}, \boldsymbol{\alpha}_{i}, \beta_{i}\right): i \in I\right\}$ in Theorem 3.1 imply that $\boldsymbol{\alpha}_{\{i\}}$ is a Zariski open inclusion, with image $\operatorname{Im} \boldsymbol{\alpha}_{\{i\}}=\operatorname{Im} \boldsymbol{\alpha}_{i}$, and (3.2) homotopy commutes for $J=\{i\}$ as (3.1) does. The only $\emptyset \neq K \subseteq J \subseteq I$ with $|J| \leqslant k=1$ are $J=K=\{i\}$, and then (3.3) homotopy commutes as $\boldsymbol{\alpha}_{J}=\boldsymbol{\alpha}_{K}=\boldsymbol{\alpha}_{\{i\}}$ and $\Phi_{J K}=$ id. This completes Hypothesis 4.1 when $k=1$. Note that our definitions $A_{\{i\}}^{\bullet}=A_{i}^{\bullet}, \boldsymbol{\alpha}_{\{i\}}=\boldsymbol{\alpha}_{i}$, and $\beta_{\{i\}}=\beta_{i}$ for $i \in I$ are as required in Theorem 3.1(i).

Next we prove the inductive step. Let $l \geqslant 1$ be given, and suppose Hypothesis 4.1 holds with $k=l$. Keeping all the data in (a),(e),(g) for $|J| \leqslant l$ the same, 
we will prove Hypothesis 4.1 with $k=l+1$. To do this, for each $J \subseteq I$ with $|J|=l+1$, we have to construct the data of finite sets $S_{J}^{n}$ for $n=-1,-2, \ldots$ in (a), and maps $\delta_{J}^{n}: S_{J}^{n} \rightarrow A_{J}^{n+1}$ in (e), and the morphism $\boldsymbol{\alpha}_{J}: \operatorname{Spec} A_{J}^{\bullet} \hookrightarrow \boldsymbol{X}$ in $(\mathrm{g})$, and then prove the claims in (f) that $\mathrm{d} \circ \mathrm{d}=0$, and in $(\mathrm{g})$ that $\boldsymbol{\alpha}_{J}$ is a Zariski open inclusion with image $\bigcap_{i \in J} \operatorname{Im} \boldsymbol{\alpha}_{i}$, and that (3.2) and (3.3) homotopy commute.

Note that as Hypothesis 4.1 involves no compatibility conditions between data for distinct $J, J^{\prime} \subseteq I$ with $|J|=\left|J^{\prime}\right|=k$, we can do this independently for each $J \subseteq I$ with $|J|=l+1$, that is, it is enough to give the proof for a single such $J$. So fix a subset $J \subseteq I$ with $|J|=l+1$.

We first define a standard form cdga $\tilde{A}_{J}^{\bullet}$ which is an approximation to the $\operatorname{cdga} A_{J}^{\bullet}$ that we want, and morphisms $\tilde{\beta}_{J}: B \rightarrow \tilde{A}_{J}^{0}, \tilde{\Phi}_{J K}: A_{K}^{\bullet} \rightarrow \tilde{A}_{J}^{\bullet}$ for all $\emptyset \neq K \subsetneq J$, so that $|K| \leqslant l$ and $A_{K}^{\bullet}$ is already defined:

- Define $\tilde{A}_{J}^{0}=A_{J}^{0}$ and $\tilde{\beta}_{J}=\beta_{J}: B \rightarrow \tilde{A}_{J}^{0}=A_{J}^{0}$ as in Hypothesis 4.1(b).

- Define $\tilde{A}_{J}^{*}$ to be the graded $\mathbb{C}$-algebra freely generated over $A_{J}^{0}$ by generators $\coprod_{\emptyset \neq K \subsetneq J} S_{K}^{n}$ in degree $n$ for $n=-1,-2, \ldots$ This is the same as for $A_{J}^{*}$ in Hypothesis 4.1(c), except that we do not include generators $S_{J}^{n}$, since $S_{J}^{n}$ is not yet defined.

- If $\emptyset \neq K \subsetneq J$, so that $A_{K}^{\bullet}$ is defined, define $\Phi_{J K}^{0}: A_{K}^{0} \rightarrow A_{J}^{0}=\tilde{A}_{J}^{0}$ as in Hypothesis 4.1(d), and define $\tilde{\Phi}_{J K}: A_{K}^{*} \rightarrow \tilde{A}_{J}^{*}$ to be the unique morphism of graded $\mathbb{C}$-algebras acting by $\Phi_{J K}^{0}$ in degree zero, and mapping $\Phi_{J K}: \gamma \mapsto \gamma$ for each $\gamma \in S_{L}^{n}$ for $\emptyset \neq L \subseteq K$ and $n=-1,-2, \ldots$

- The differential d : $\tilde{A}_{J}^{*} \rightarrow \tilde{A}_{J}^{*+1}$ in the cdga $\tilde{A}_{J}^{\bullet}=\left(\tilde{A}_{J}^{*}, \mathrm{~d}\right)$ is determined uniquely as in (4.2) by

$$
\mathrm{d} \gamma=\tilde{\Phi}_{J K} \circ \delta_{K}^{n}(\gamma) \text { for all } \emptyset \neq K \subsetneq J, n \leqslant-1 \text { and } \gamma \in S_{K}^{n} .
$$

Then $\tilde{\Phi}_{J K}: A_{K}^{\bullet} \rightarrow \tilde{A}_{J}^{\bullet}$ is a cdga morphism for all $\emptyset \neq K \subsetneq J$, as in Hypothesis 4.1(f) for $\Phi_{J K}$.

That is, $\tilde{A}_{J}^{\bullet}$ is the colimit in the ordinary category $\mathbf{c d g a}_{\mathbb{C}}$ of the commutative diagram $\Gamma$ with vertices the objects $B$ and $A_{K}^{\bullet}$ for all $K$ with $\emptyset \neq K \subsetneq J$, and edges the morphisms $\beta_{K}: B \rightarrow A_{K}^{\bullet}$ and $\Phi_{K_{1} K_{2}}: A_{K_{2}}^{\bullet} \rightarrow A_{K_{1}}^{\bullet}$ for $\emptyset \neq K_{2} \subsetneq$ $K_{1} \subsetneq J$, and $\tilde{\beta}_{J}: B \rightarrow \tilde{A}_{J}^{\bullet}, \tilde{\Phi}_{J K}: A_{K}^{\bullet} \rightarrow \tilde{A}_{J}^{\bullet}$ are the projections to the colimit. Since all the morphisms in $\Gamma$ are almost-free in negative degrees and smooth in degree 0 , these morphisms are sufficiently cofibrant to compute the homotopy colimits as well. Indeed, having such a morphism $A^{\bullet} \rightarrow C^{\bullet}$ we can factor it into $A^{\bullet} \rightarrow A^{\bullet} \underset{A^{0}}{\otimes} C^{0} \rightarrow C^{\bullet}$. Each one of these morphisms is flat, and hence homotopy pullbacks can be computed without resolving. Finally we notice that the colimit of the entire diagram $\Gamma$ can be calculated as a sequence of pullbacks. So $\tilde{A}_{J}^{\bullet}$ is also the homotopy colimit of $\Gamma$ in the $\infty$-category $\operatorname{cdga}_{\mathbb{C}}^{\infty}$. Hence $\operatorname{Spec} \tilde{A}_{J}^{\bullet}$ is the homotopy limit of Spec $\Gamma$ in the $\infty$-category $\mathbf{d S c h}_{\mathbb{C}}$.

For $\emptyset \neq K \subsetneq J$, consider $\bigcap_{i \in K} \operatorname{Im} \boldsymbol{\alpha}_{i}$ as an open derived $\mathbb{C}$-subscheme of $\boldsymbol{X}$. Then by Hypothesis $4.1(\mathrm{~g}), \boldsymbol{\alpha}_{K}: \operatorname{Spec} A_{K}^{\bullet} \rightarrow \bigcap_{i \in K} \operatorname{Im} \boldsymbol{\alpha}_{i}$ is an equivalence in 
$\mathbf{d S c h}_{\mathbb{C}}$. We also have the open derived $\mathbb{C}$-subscheme $\bigcap_{i \in J} \operatorname{Im} \boldsymbol{\alpha}_{i}$ in $\boldsymbol{X}$, which is affine by Definition 2.6 as $\boldsymbol{X}$ has affine diagonal and $\operatorname{Im} \boldsymbol{\alpha}_{i} \simeq \operatorname{Spec} A_{i}^{\bullet}$ is affine for $i \in J$. Thus we may choose a standard form cdga $\hat{A}_{J}^{\bullet}$ and an equivalence $\hat{\alpha}_{J}: \operatorname{Spec} \hat{A}_{J}^{\bullet} \hookrightarrow \bigcap_{i \in J} \operatorname{Im} \boldsymbol{\alpha}_{i}$.

Define morphisms $\hat{\boldsymbol{\beta}}_{J}: \operatorname{Spec} \hat{A}_{J}^{\bullet} \rightarrow Z=\operatorname{Spec} B$ by $\boldsymbol{\beta}_{J}=\boldsymbol{\pi} \circ \hat{\boldsymbol{\alpha}}_{\boldsymbol{J}}$, and $\hat{\phi}_{J K}: \operatorname{Spec} \hat{A}_{J}^{\bullet} \rightarrow \operatorname{Spec} A_{K}^{\bullet}$ for $\emptyset \neq K \subsetneq J$ to be the composition

$$
\operatorname{Spec} \hat{A}_{J}^{\bullet} \stackrel{\hat{\alpha}_{J}}{\longrightarrow} \bigcap_{i \in J} \operatorname{Im} \boldsymbol{\alpha}_{i} \longrightarrow \bigcap_{i \in K} \operatorname{Im} \boldsymbol{\alpha}_{i} \stackrel{\boldsymbol{\alpha}_{K}^{-1}}{\longrightarrow} \operatorname{Spec} A_{K}^{\bullet},
$$

where $\boldsymbol{\alpha}_{K}^{-1}$ is a quasi-inverse for the equivalence $\boldsymbol{\alpha}_{K}: \operatorname{Spec} A_{K}^{\bullet} \rightarrow \bigcap_{i \in K} \operatorname{Im} \boldsymbol{\alpha}_{i}$.

Now by the homotopy limit property of $\operatorname{Spec} \tilde{A}_{J}^{\bullet}$, there exists a morphism $\boldsymbol{\psi}: \operatorname{Spec} \hat{A}_{J}^{\bullet} \rightarrow \operatorname{Spec} \tilde{A}_{J}^{\bullet}$ in $\mathbf{d} \mathbf{S c h} \mathbb{C}_{\mathbb{C}}$ unique up to homotopy, with homotopies $\hat{\boldsymbol{\beta}}_{J} \simeq \operatorname{Spec} \tilde{\beta}_{J} \circ \boldsymbol{\psi}$ and $\hat{\boldsymbol{\phi}}_{J K} \simeq \operatorname{Spec} \tilde{\Phi}_{J K} \circ \boldsymbol{\psi}$ for $\emptyset \neq K \subsetneq J$. We can then write $\boldsymbol{\psi} \simeq \operatorname{Spec} \Psi$ for $\Psi: \tilde{A}_{J}^{\bullet} \rightarrow \hat{A}_{J}^{\bullet}$ a morphism in $\mathbf{c d g a}_{\mathbb{C}}^{\infty}$, unique up to homotopy. However, we do not yet know that $\Psi$ descends to a morphism in cdga $_{\mathbb{C}}$. The definitions of $\hat{\boldsymbol{\beta}}_{J}, \hat{\boldsymbol{\phi}}_{J K}$ and $\boldsymbol{\psi} \simeq \operatorname{Spec} \Psi$ give homotopies

$$
\begin{aligned}
\pi & \circ \hat{\boldsymbol{\alpha}}_{J} \simeq \operatorname{Spec} \tilde{\beta}_{J} \circ \operatorname{Spec} \Psi: \operatorname{Spec} \hat{A}_{J}^{\bullet} \longrightarrow Z, \\
\hat{\boldsymbol{\alpha}}_{J} & \simeq \boldsymbol{\alpha}_{K} \circ \operatorname{Spec} \tilde{\Phi}_{J K} \circ \operatorname{Spec} \Psi: \operatorname{Spec} \hat{A}_{J}^{\bullet} \longrightarrow X, \emptyset \neq K \subsetneq J .
\end{aligned}
$$

Consider the composition of morphisms of classical $\mathbb{C}$-algebras

$$
A_{J}^{0} \rightleftharpoons \tilde{A}_{J}^{0} \longrightarrow H^{0}\left(\tilde{A}_{J}^{\bullet}\right) \stackrel{H^{0}(\Psi)}{\longrightarrow} H^{0}\left(\hat{A}_{J}^{\bullet}\right) .
$$

Here Spec $H^{0}(\Psi)$ is the natural morphism

$$
\operatorname{Spec} H^{0}(\Psi): X_{J} \longrightarrow \prod_{\emptyset \neq K \subsetneq J}^{\text {over } Z} X_{K},
$$

writing $X_{K}$ for the open $\mathbb{C}$-subscheme $\bigcap_{k \in K} t_{0}\left(\operatorname{Im} \boldsymbol{\alpha}_{k}\right)$ in $X$. This is the restriction of the multidiagonal $\Delta_{X}^{2^{|J|-2}}: X \rightarrow X \times_{Z} X \times_{Z} \cdots \times_{Z} X$, with $2^{|J|-2}$ copies of $X$ on the right. As $X$ is separated, $\Delta_{X}^{2}: X \rightarrow X \times_{Z} X$ is a closed immersion, so $\Delta_{X}^{2^{|J|-2}}$ is a closed immersion. Also the domain $X_{J}$ of (4.5) is the preimage under $\Delta_{X}^{2^{|J|-2}}$ of the target, since $X_{J}=\bigcap_{\emptyset \neq K \subset J} X_{K}$ as $|J| \geqslant 2$.

Hence (4.5) is a closed immersion, so $H^{0}(\Psi)$ in (4.4) is surjective. Also $\tilde{A}_{J}^{0} \rightarrow H^{0}\left(\tilde{A}_{J}^{\bullet}\right)$ is surjective, so the composition (4.4) is surjective. Therefore we can replace $\hat{A}_{J}^{\bullet}$ by an equivalent object in $\mathbf{c d g a}_{\mathbb{C}}^{\infty}$, such that $\hat{A}_{J}^{0}=\tilde{A}_{J}^{0}$, and the following homotopy commutes in $\mathbf{c d g a}_{\mathbb{C}}^{\infty}$ :

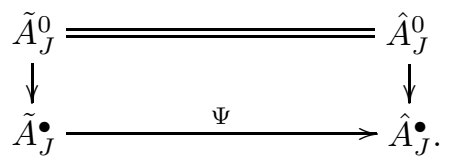

Now $\Psi: \tilde{A}_{J}^{\bullet} \rightarrow \hat{A}_{J}^{\bullet}$ is a morphism in $\mathbf{c d g a}_{\mathbb{C}}^{\infty}$. For this to descend to a morphism in $\mathbf{c d g a}_{\mathbb{C}}$, the simplest condition is that $\tilde{A}_{J}^{\bullet}$ should be cofibrant and $\hat{A}_{J}^{\bullet}$ fibrant in the model category $\mathbf{c d g a}_{\mathbb{C}}$. Here $\hat{A}_{J}^{\bullet}$ is fibrant, as all objects are, 
but $\tilde{A}_{J}^{\bullet}$ may not be cofibrant, i.e. a retract of an almost-free cdga. However, $\tilde{A}_{J}^{\bullet}$ is cofibrant as an $\tilde{A}_{J}^{0}$-algebra, as it is free in negative degrees, and (4.6) says that $\Psi$ does descend to a morphism in cdga $\mathbb{C}_{\mathbb{C}}$ in degree 0 . Together these imply that $\Psi$ descends to a morphism $\Psi: \tilde{A}_{J}^{\bullet} \rightarrow \hat{A}_{J}^{\bullet}$ in $\mathbf{c d g a}_{\mathbb{C}}$.

Next, by induction on decreasing $n=-1,-2, \ldots$ we will choose the data $S_{J}^{n}, \delta_{J}^{n}$ in Hypothesis 4.1(a),(e). Here is our inductive hypothesis:

Hypothesis 4.3. Let $N=0,-1,-2, \ldots$ be given. Then:

(a) We are given finite subsets $S_{J}^{n}$ for $n=-1,-2, \ldots, N$. Write $A_{J, N}^{*}=$ $\tilde{A}_{J}^{*}\left[S_{J}^{1}, \ldots, S_{J}^{N}\right]$ for the graded $\mathbb{C}$-algebra freely generated over $\tilde{A}_{J}^{*}$ by the sets of extra generators $S_{J}^{n}$ in degree $n$ for all $n=-1,-2, \ldots, N$.

(b) We are given maps $\delta_{J}^{n}: S_{J}^{n} \rightarrow A_{J, N}^{n+1}$ for $n=-1,-2, \ldots, N$. Define d : $A_{J, N}^{*} \rightarrow A_{J, N}^{*+1}$ uniquely by the conditions that d satisfies the Leibnitz rule, and $\mathrm{d}$ is as in $\tilde{A}_{J}^{\bullet}=\left(\tilde{A}_{J}^{*}, \mathrm{~d}\right)$ on $\tilde{A}_{J}^{*} \subseteq A_{J, N}^{*}$, and on the extra generators $\gamma \in S_{J}^{n}$ for $n=-1,-2, \ldots, N$, we have $\mathrm{d} \gamma=\delta_{J}^{n}(\gamma) \in A_{J, N}^{n+1}$. We require that $\mathrm{d} \circ \mathrm{d}=0: A_{J, N}^{*} \rightarrow A_{J, N}^{*+2}$, so that $A_{J, N}^{\bullet}=\left(A_{J, N}^{*}, \mathrm{~d}\right)$ is a cdga.

(c) We are given maps $\xi_{J}^{n}: S_{J}^{n} \rightarrow \hat{A}_{J}^{n}$ for $n=-1,-2, \ldots, N$.

Define $\Xi_{N}: A_{J, N}^{*} \rightarrow \hat{A}_{J}^{*}$ to be the morphism of graded $\mathbb{C}$-algebras such that $\Xi_{N}=\Psi$ on $\tilde{A}_{J}^{*} \subseteq A_{J, N}^{*}$, and on the extra generators $\gamma \in S_{J}^{n}$ for $n=-1,-2, \ldots, N$, we have $\Xi_{N}(\gamma)=\xi_{J}^{n}(\gamma) \in \hat{A}_{J, N}^{n}$.

We require that $\Xi_{N} \circ \mathrm{d}=\mathrm{d} \circ \Xi_{N}: A_{J, N}^{*} \rightarrow \hat{A}_{J}^{*+1}$, so that $\Xi_{N}: A_{J, N}^{\bullet} \rightarrow \hat{A}_{J}^{\bullet}$ is a cdga morphism.

We also require that $H^{n}\left(\Xi_{N}\right): H^{n}\left(A_{J, N}^{\bullet}\right) \rightarrow H^{n}\left(\hat{A}_{J}^{\bullet}\right)$ should be an isomorphism for $n=0,-1,-2, \ldots, N+1$, and surjective for $n=N$.

For the first step $N=0$, there is no data $S_{J}^{n}, \delta_{J}^{n}, \xi_{J}^{n}$, and $A_{J, 0}^{\bullet}=\tilde{A}_{J}^{\bullet}$, and $\Xi_{0}=\Psi$, and the only thing to prove is that $H^{0}(\Psi): H^{0}\left(\tilde{A}_{J}^{\bullet}\right) \rightarrow H^{0}\left(\hat{A}_{J}^{\bullet}\right)$ is surjective, which holds as $\Psi^{0}=\mathrm{id}: \tilde{A}_{J}^{0} \rightarrow \tilde{A}_{J}^{0}=\hat{A}_{J}^{0}$ from above. So Hypothesis 4.3 holds for $N=0$.

For the inductive step, let $m=0,-1,-2, \ldots$ be given, and suppose Hypothesis 4.3 holds with $N=m$. Keeping all the data $S_{J}^{n}, \delta_{J}^{n}, \xi_{J}^{n}$ for $n=-1, \ldots, m$ the same, we will prove Hypothesis 4.3 with $N=m-1$. Note that with $S_{J}^{-1}, \ldots, S_{J}^{m}$ the same, the graded $\mathbb{C}$-algebras $A_{J, m}^{*}, A_{J, m-1}^{*}$ agree in degrees $0,-1, \ldots, m$, so it makes sense to say that $\delta_{J}^{n}: S_{J}^{n} \rightarrow A_{J, m}^{n+1}$ and $\delta_{J}^{n}: S_{J}^{n} \rightarrow A_{J, m-1}^{n+1}$ are equal for $n=-1,-2, \ldots, m$. We must choose data $S_{J}^{m-1}, \delta_{J}^{m-1}: S_{J}^{m-1} \rightarrow A_{J, m-1}^{m}$ and $\xi_{J}^{m-1}: S_{J}^{m-1} \rightarrow \hat{A}_{J}^{m-1}$, and verify the last two conditions of Hypothesis 4.3(c).

Choose a finite subset $\dot{S}_{J}^{m-1}$ of $\operatorname{Ker}\left(H^{m}\left(\Xi_{m}\right): H^{m}\left(A_{J, m}^{\bullet}\right) \rightarrow H^{m}\left(\hat{A}_{J}^{\bullet}\right)\right)$ which generates $\operatorname{Ker}(\cdots)$ as an $H^{0}\left(A_{J, m}^{\bullet}\right)$-module, and a finite subset $\ddot{S}_{J}^{m-1}$ of $H^{m-1}\left(\hat{A}_{J}^{\bullet}\right)$ such that $\ddot{S}_{J}^{m-1}$ and $\operatorname{Im}\left(H^{m-1}\left(\Xi_{m}\right): H^{m-1}\left(A_{J, m}^{\bullet}\right) \rightarrow H^{m-1}\left(\hat{A}_{J}^{\bullet}\right)\right)$ generate $H^{m-1}\left(\hat{A}_{J}^{\bullet}\right)$ as an $H^{0}\left(\hat{A}_{J}^{\bullet}\right)$-module. Finite subsets suffice in each case 
since $A_{J, m}^{\bullet}, \hat{A}_{J}^{\bullet}$ are of standard form, so that $H^{n}\left(A_{J, m}^{\bullet}\right), H^{n}\left(\hat{A}_{J}^{\bullet}\right)$ are finitely generated over $H^{0}\left(A_{J, m}^{\bullet}\right), H^{0}\left(\hat{A}_{J}^{\bullet}\right)$ for all $n$. Set $S_{J}^{m-1}=\dot{S}_{J}^{m-1} \amalg \ddot{S}_{J}^{m-1}$.

Then Hypothesis 4.3(a) defines $A_{J, m-1}^{*}$ as a graded $\mathbb{C}$-algebra, with $A_{J, m-1}^{n}$ $=A_{J, m}^{n}$ in degrees $n \geqslant m$. For all $\gamma \in \dot{S}_{J}^{m-1}$, choose a representative $\delta_{J}^{m-1}(\gamma)$ in $A_{J, m-1}^{m}=A_{J, m}^{m}$ for the cohomology class $\gamma$ in $H^{m}\left(A_{J, m}^{\bullet}\right)$, so that $\mathrm{d}\left(\delta_{J}^{m-1}(\gamma)\right)=0$ in $A_{J, m}^{m+1}$. Define $\delta_{J}^{m-1}(\gamma)=0$ in $A_{J, m-1}^{m}$ for all $\gamma \in \ddot{S}_{J}^{m-1}$. This defines $\delta_{J}^{m-1}: S_{J}^{m-1} \rightarrow A_{J, m-1}^{m}$ in Hypothesis 4.3(b), and hence d : $A_{J, m-1}^{*} \rightarrow A_{J, m-1}^{*+1}$.

To see that $\mathrm{d} \circ \mathrm{d}=0: A_{J, m-1}^{*} \rightarrow A_{J, m-1}^{*+2}$, note that $A_{J, m-1}^{*}=A_{J, m}^{*}\left[S_{J}^{m-1}\right]$, so d on $A_{J, m-1}^{*}$ is determined by d on $A_{J, m}^{*}$, which already satisfies $\mathrm{d} \circ \mathrm{d}=0$ by induction, and d on the extra generators $S_{J}^{m-1}$, which satisfy d $\circ \mathrm{d}=0$ as for $\gamma \in \dot{S}_{J}^{m-1}$ we have $\mathrm{d} \circ \mathrm{d} \gamma=\mathrm{d}\left(\delta_{J}^{m-1}(\gamma)\right)=0$, and for $\gamma \in \ddot{S}_{J}^{m-1}$ we have $\mathrm{d} \gamma=0$ so $\mathrm{d} \circ \mathrm{d} \gamma=0$. Hence $A_{J, m-1}^{\bullet}=\left(A_{J, m-1}^{*}, \mathrm{~d}\right)$ is a cdga, as we have to prove.

For all $\gamma \in \dot{S}_{J}^{m-1}$, as $\delta_{J}^{m-1}(\gamma) \in A_{J, m}^{m}$ represents a cohomology class in $\operatorname{Ker}\left(H^{m}\left(\Xi_{m}\right): H^{m}\left(A_{J, m}^{\bullet}\right) \rightarrow H^{m}\left(\hat{A}_{J}^{\bullet}\right)\right)$, we see that $\Xi_{m} \circ \delta_{J}^{m-1}(\gamma)$ is exact in $\hat{A}_{J}^{\bullet}$, so we can choose an element $\xi_{J}^{m-1}(\gamma) \in \hat{A}_{J}^{m-1}$ with $\mathrm{d} \circ \xi_{J}^{m-1}(\gamma)=\Xi_{m} \circ$ $\delta_{J}^{m-1}(\gamma)$. For all $\gamma \in \ddot{S}_{J}^{m-1} \subset H^{m-1}\left(\hat{A}_{J}^{\bullet}\right)$, choose an element $\xi_{J}^{m-1}(\gamma) \in \hat{A}_{J}^{m-1}$ representing $\gamma$, so that $\mathrm{d} \circ \xi_{J}^{m-1}(\gamma)=0$. This defines $\xi_{J}^{m-1}: S_{J}^{m-1} \rightarrow \hat{A}_{J}^{m-1}$.

Hypothesis 4.3(c) now defines $\Xi_{m-1}: A_{J, m-1}^{*} \rightarrow \hat{A}_{J}^{*}$. To prove that $\Xi_{m-1} \circ$ $\mathrm{d}=\mathrm{d} \circ \Xi_{m-1}$, note that $A_{J, m-1}^{*}=A_{J, m}^{*}\left[S_{J}^{m-1}\right]$, and on $A_{J, m}^{*} \subseteq A_{J, m-1}^{*}$ we have $\Xi_{m-1}=\Xi_{m}$, and $\Xi_{m} \circ \mathrm{d}=\mathrm{d} \circ \Xi_{m}$ by induction. So it is enough to prove that $\Xi_{m-1} \circ \mathrm{d}(\gamma)=\mathrm{d} \circ \Xi_{m-1}(\gamma)$ for all $\gamma \in S_{J}^{m-1}$. If $\gamma \in \dot{S}_{J}^{m-1}$ then

$$
\Xi_{m-1} \circ \mathrm{d}(\gamma)=\Xi_{m-1} \circ \delta_{J}^{m-1}(\gamma)=\Xi_{m} \circ \delta_{J}^{m-1}(\gamma)=\mathrm{d} \circ \xi_{J}^{m-1}(\gamma)=\mathrm{d} \circ \Xi_{m-1}(\gamma),
$$

as we want. Similarly, if $\gamma \in \ddot{S}_{J}^{m-1}$ then

$$
\Xi_{m-1} \circ \mathrm{d}(\gamma)=\Xi_{m-1} \circ \delta_{J}^{m-1}(\gamma)=0=\mathrm{d} \circ \xi_{J}^{m-1}(\gamma)=\mathrm{d} \circ \Xi_{m-1}(\gamma) .
$$

Therefore $\Xi_{m-1} \circ \mathrm{d}=\mathrm{d} \circ \Xi_{m-1}$, and $\Xi_{m-1}: A_{J, m-1}^{\bullet} \rightarrow \hat{A}_{J}^{\bullet}$ is a cdga morphism.

Finally we have to show that $H^{n}\left(\Xi_{m-1}\right): H^{n}\left(A_{J, m-1}^{\bullet}\right) \rightarrow H^{n}\left(\hat{A}_{J}^{\bullet}\right)$ is be an isomorphism for $n=-1,-2, \ldots, m$, and surjective for $n=m-1$. Since $\Xi_{m}: A_{J, m}^{\bullet} \rightarrow \hat{A}_{J}^{\bullet}$ and $\Xi_{m-1}: A_{J, m-1}^{\bullet} \rightarrow \hat{A}_{J}^{\bullet}$ coincide in degrees $0,-1, \ldots, m$, in cohomology they coincide in degrees $0,-1, \ldots, m+1$, so $H^{n}\left(\Xi_{m-1}\right)$ is an isomorphism for $n=0,-1, \ldots, m+1$ as $H^{n}\left(\Xi_{m}\right)$ is, by induction.

As $H^{m}\left(\Xi_{m}\right): H^{m}\left(A_{J, m}^{\bullet}\right) \rightarrow H^{m}\left(\hat{A}_{J}^{\bullet}\right)$ is surjective, and the added generators $\dot{S}_{J}^{m-1}$ in $A_{J, m-1}^{\bullet}$ span $\operatorname{Ker}\left(H^{m}\left(\Xi_{m}\right)\right)$, adding $\dot{S}_{J}^{m-1}$ makes $H^{m}\left(\Xi_{m-1}\right)$ : $H^{m}\left(A_{J, m-1}^{\bullet}\right) \rightarrow H^{m}\left(\hat{A}_{J}^{\bullet}\right)$ into an isomorphism. Also, since the added generators $\ddot{S}_{J}^{m-1}$ together with $\operatorname{Im}\left(H^{m-1}\left(\Xi_{m}\right)\right)$ generate $H^{m-1}\left(\hat{A}_{J}^{\bullet}\right)$, adding $\ddot{S}_{J}^{m-1}$ makes $H^{m-1}\left(\Xi_{m-1}\right): H^{m-1}\left(A_{J, m-1}^{\bullet}\right) \rightarrow H^{m-1}\left(\hat{A}_{J}^{\bullet}\right)$ surjective.

This proves Hypothesis 4.3 for $N=m-1$, so by induction Hypothesis 4.3

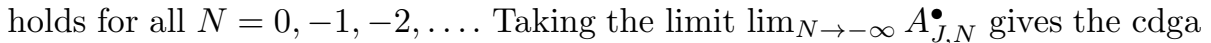
$A_{J}^{\bullet}$ defined in Hypothesis 4.1 using the data $S_{J}^{n}, \delta_{J}^{n}$ for all $n=-1,-2, \ldots$ from Hypothesis 4.3(a),(b) as $N \rightarrow-\infty$. The data $\xi_{J}^{n}$ for $n=-1,-2, \ldots$ from part 
(c) defines a morphism $\Xi=\lim _{N \rightarrow-\infty} \Xi_{N}: A_{J}^{\bullet} \rightarrow \hat{A}_{J}^{\bullet}$, where $\Xi, A_{J}^{\bullet}$ agree with $\Xi_{N}, A_{J, N}^{\bullet}$ in degrees $0,-1, \ldots, N$ for all $N \leqslant 0$.

Hence $H^{n}(\Xi): H^{n}\left(A_{J}^{\bullet}\right) \rightarrow H^{n}\left(\hat{A}_{J}^{\bullet}\right)$ agrees with $H^{n}\left(\Xi_{N}\right): H^{n}\left(A_{J, N}^{\bullet}\right) \rightarrow$ $H^{n}\left(\hat{A}_{J}^{\bullet}\right)$ for all $n=0,-1, \ldots, N+1$, so $H^{n}(\Xi)$ is an isomorphism for all $n \leqslant 0$ by Hypothesis 4.3 (c), and $\Xi: A_{J}^{\bullet} \rightarrow \hat{A}_{J}^{\bullet}$ is a quasi-isomorphism in cdga $\mathbf{C}_{\mathbb{C}}$, and hence an equivalence in $\operatorname{cdga}_{\mathbb{C}}^{\infty}$. Thus $\operatorname{Spec} \Xi: \operatorname{Spec} \hat{A}_{J}^{\bullet} \rightarrow \operatorname{Spec} A_{J}^{\bullet}$ is an equivalence in $\mathbf{d S c h}_{\mathbb{C}}$. So we can choose a quasi-inverse $\chi: \mathbf{S p e c} A_{J}^{\bullet} \rightarrow \mathbf{S p e c} \hat{A}_{J}^{\bullet}$ in $\mathbf{d} \mathbf{S c h} \mathbf{C}_{\mathbb{C}}$.

Write $\iota: \tilde{A}_{J}^{\bullet} \hookrightarrow A_{J}^{\bullet}$ for the inclusion. Then $\Psi=\Xi \circ \iota: \tilde{A}_{J}^{\bullet} \rightarrow \hat{A}_{J}^{\bullet}$, since $\left.\Xi_{N}\right|_{\tilde{A}_{J}^{\bullet}}=\Psi$, so taking the limit $N \rightarrow-\infty$ gives $\left.\Xi\right|_{\tilde{A}_{J}^{\bullet}}=\Psi$. Also the definitions of $\beta_{J}: B \rightarrow A_{J}^{\bullet}$ and $\Phi_{J K}: A_{K}^{\bullet} \rightarrow A_{J}^{\bullet}$ for $\emptyset \neq K \subsetneq J$ in Hypothesis 4.1(b),(d) satisfy $\beta_{J}=\iota \circ \tilde{\beta}_{J}$ and $\Phi_{J K}=\iota \circ \tilde{\Phi}_{J K}$.

Define $\boldsymbol{\alpha}_{J}=\hat{\boldsymbol{\alpha}}_{J} \circ \chi: \operatorname{Spec} A_{J}^{\bullet} \rightarrow \boldsymbol{X}$. Since $\hat{\boldsymbol{\alpha}}_{J}$ is a Zariski open inclusion with image $\bigcap_{i \in J} \operatorname{Im} \boldsymbol{\alpha}_{i}$, and $\boldsymbol{\chi}$ is an equivalence, $\boldsymbol{\alpha}_{J}: \operatorname{Spec} A_{J}^{\bullet} \rightarrow \boldsymbol{X}$ is a Zariski open inclusion with image $\bigcap_{i \in J} \operatorname{Im} \boldsymbol{\alpha}_{i}$, as in Hypothesis 4.1(g). Then we have

$$
\begin{aligned}
\boldsymbol{\pi} \circ \boldsymbol{\alpha}_{J} & =\boldsymbol{\pi} \circ \hat{\boldsymbol{\alpha}}_{J} \circ \chi \simeq \operatorname{Spec} \tilde{\beta}_{J} \circ \operatorname{Spec} \Psi \circ \chi \\
& \simeq \operatorname{Spec} \tilde{\beta}_{J} \circ \operatorname{Spec} \iota \circ \operatorname{Spec} \Xi \circ \chi \simeq \operatorname{Spec} \tilde{\beta}_{J} \circ \operatorname{Spec} \iota=\operatorname{Spec} \beta_{J},
\end{aligned}
$$

using (4.3) in the second step, $\Psi=\Xi \circ \iota$ in the third, Spec $\Xi$, $\chi$ quasi-inverse in the fourth, and $\beta_{J}=\iota \circ \tilde{\beta}_{J}$ in the fifth. Thus (3.2) homotopy commutes.

Similarly, if $\emptyset \neq K \subsetneq J$ then

$$
\begin{aligned}
\boldsymbol{\alpha}_{J} & =\hat{\boldsymbol{\alpha}}_{J} \circ \chi \simeq \boldsymbol{\alpha}_{K} \circ \operatorname{Spec} \tilde{\Phi}_{J K} \circ \operatorname{Spec} \Psi \circ \chi \\
& \simeq \boldsymbol{\alpha}_{K} \circ \operatorname{Spec} \tilde{\Phi}_{J K} \circ \operatorname{Spec} \iota \circ \operatorname{Spec} \Xi \circ \chi \simeq \boldsymbol{\alpha}_{K} \circ \operatorname{Spec} \Phi_{J K}
\end{aligned}
$$

using (4.3) in the second step, $\Psi=\Xi \circ \iota$ in the third, and $\Phi_{J K}=\iota \circ \tilde{\Phi}_{J K}$ and Spec $\Xi, \chi$ quasi-inverse in the fourth. Hence (3.3) homotopy commutes.

This proves that Hypothesis 4.1 holds with $k=l+1$, and completes the inductive step begun shortly after Remark 4.2. Hence by induction, Hypothesis 4.1 holds for all $k=1,2, \ldots$, so Hypothesis 4.1 holds for $k=\infty$. Theorem 3.1 follows, since all the conclusions of Theorem 3.1(i),(ii) are either part of Hypothesis 4.1, or for $A_{\{i\}}^{\bullet}=A_{i}^{\bullet}, \boldsymbol{\alpha}_{\{i\}}=\boldsymbol{\alpha}_{i}, \beta_{\{i\}}=\beta_{i}$ in part (i) were included in the first step of the induction. This completes the proof.

\section{$5 \quad$ Proof of Theorem 3.7}

\subsection{Theorem 3.7(a): (*) is an open condition}

Suppose $\boldsymbol{X}, \omega_{\boldsymbol{X}}^{*}, A^{\bullet}, \boldsymbol{\alpha}, V, E, F, s, t, \psi$ are as in Definition [3.6, and suppose that $U \subseteq V$ is open, $E^{-}$is a real vector subbundle of $\left.E\right|_{U}$, and $v \in s^{-1}(0) \cap U$, such that the assumptions on $\left.E^{-}\right|_{v}$ in condition $(*)$ hold at $v$. We must show that these assumptions also hold for all $v^{\prime}$ in an open neighbourhood of $v$ in $s^{-1}(0) \cap U$. Suppose for a contradiction that this is false. Then we can choose a sequence $\left(v_{i}\right)_{i=1}^{\infty}$ in $s^{-1}(0) \cap U$ such that $v_{i} \rightarrow v$ as $i \rightarrow \infty$, and the assumptions on $\left.E^{-}\right|_{v_{i}}$ in $(*)$ do not hold for any $i=1,2, \ldots$. 
By passing to a subsequence of $\left(v_{i}\right)_{i=1}^{\infty}$, we can assume that $\left.\operatorname{dim} \operatorname{Im} \mathrm{d} s\right|_{v_{i}}$ and $\left.\operatorname{dim} \operatorname{Ker} t\right|_{v_{i}}$ are independent of $i=1,2, \ldots$ By trivializing $E$ near $v$, we can regard $\left(\left.\operatorname{Im~d} s\right|_{v_{i}}\right)_{i=1}^{\infty}$ and $\left(\left.\operatorname{Ker} t\right|_{v_{i}}\right)_{i=1}^{\infty}$ as sequences in complex Grassmannians, which are compact. Thus, passing to a subsequence of $\left(v_{i}\right)_{i=1}^{\infty}$, we can assume they converge, and there are complex vector subspaces $I_{v},\left.K_{v} \subseteq E\right|_{v}$ such that $\left.\operatorname{Imd} s\right|_{v_{i}} \rightarrow I_{v}$ and $\left.\operatorname{Ker} t\right|_{v_{i}} \rightarrow K_{v}$ as $i \rightarrow \infty$.

As $t \circ \mathrm{d} s=0$ on $s^{-1}(0)$ we have $\left.\left.\operatorname{Imd} s\right|_{v_{i}} \subseteq \operatorname{Ker} t\right|_{v_{i}}$, and so $I_{v} \subseteq K_{v}$. Also $\left.\operatorname{Imd} s\right|_{v} \subseteq I_{v}$, since if $w \in T_{v} V$ we can find $w_{i} \in T_{v_{i}} V$ with $w_{i} \rightarrow w$ as $i \rightarrow \infty$, and then $\left.\left.\mathrm{d} s\right|_{v_{i}}\left(w_{i}\right) \rightarrow \mathrm{d} s\right|_{v}(w)$ as $i \rightarrow \infty$. Similarly $\left.K_{v} \subseteq \operatorname{Ker} t\right|_{v}$.

We now have a quotient vector space $\left(\left.\operatorname{Ker} t\right|_{v}\right) /\left(\left.\operatorname{Im} \mathrm{d} s\right|_{v}\right)$, which as in (3.21) carries a nondegenerate quadratic form $\tilde{Q}_{v}$. There are subspaces $I_{v} /\left(\left.\operatorname{Im} \mathrm{d} s\right|_{v}\right) \subseteq$ $K_{v} /\left(\left.\operatorname{Imd} s\right|_{v}\right) \subseteq\left(\left.\operatorname{Ker} t\right|_{v}\right) /\left(\left.\operatorname{Imd} s\right|_{v}\right)$. Also, for each $i=1,2, \ldots$ we have quotient space $\left(\left.\operatorname{Ker} t\right|_{v_{i}}\right) /\left(\left.\operatorname{Im} d s\right|_{v_{i}}\right)$ with quadratic forms $\tilde{Q}_{v_{i}}$. As $i \rightarrow \infty$ we have

$$
\left(\left.\operatorname{Ker} t\right|_{v_{i}}\right) /\left(\left.\operatorname{Im} \mathrm{d} s\right|_{v_{i}}\right) \longrightarrow K_{v} / I_{v} \cong\left[K_{v} /\left(\left.\operatorname{Im} \mathrm{d} s\right|_{v}\right)\right] /\left[I_{v} /\left(\left.\operatorname{Im} \mathrm{d} s\right|_{v}\right)\right] .
$$

One can prove using a representative $\omega_{A} \bullet$ for $\boldsymbol{\alpha}^{*}\left(\omega_{\boldsymbol{X}}^{0}\right)$ that

$$
I_{v} /\left(\left.\operatorname{Imd} s\right|_{v}\right)=\left\{e \in\left(\left.\operatorname{Ker} t\right|_{v}\right) /\left(\left.\operatorname{Imd} s\right|_{v}\right): \tilde{Q}_{v}(e, k)=0 \forall k \in K_{v} /\left(\left.\operatorname{Im~d} s\right|_{v}\right)\right\},
$$

that is, $I_{v} /\left(\left.\operatorname{Imd} s\right|_{v}\right)$ and $K_{v} /\left(\left.\operatorname{Imd} s\right|_{v}\right)$ are orthogonal subspaces w.r.t. $\tilde{Q}_{v}$. Hence the restriction of $\tilde{Q}_{v}$ to $K_{v} /\left(\left.\operatorname{Imd} s\right|_{v}\right)$ is null along $I_{v} /\left(\left.\operatorname{Im} \mathrm{d} s\right|_{v}\right)$, and descends to a nondegenerate quadratic form $\check{Q}_{v}$ on $\left[K_{v} /\left(\left.\operatorname{Im} \mathrm{d} s\right|_{v}\right)\right] /\left[I_{v} /\left(\left.\operatorname{Im} \mathrm{d} s\right|_{v}\right)\right]$ $\cong K_{v} / I_{v}$. Then under the limit (5.1), we have $\tilde{Q}_{v_{i}} \rightarrow \check{Q}_{v}$ as $i \rightarrow \infty$.

By $(*)$ for $\left(U, E^{-}\right)$at $v$, we have $\left.\operatorname{Im}\left(\left.\mathrm{d} s\right|_{v}\right) \cap E^{-}\right|_{v}=\{0\}$, and the map $\Pi_{v}:\left.E^{-}\right|_{v} \cap \operatorname{Ker}\left(\left.t\right|_{v}\right) \rightarrow\left(\left.\operatorname{Ker} t\right|_{v}\right) /\left(\left.\operatorname{Imd} s\right|_{v}\right)$ in (3.24) has image $\operatorname{Im} \Pi_{v}$ of half the total dimension, with $\operatorname{Re} \tilde{Q}_{v}$ negative definite on $\operatorname{Im} \Pi_{v}$. Since $\tilde{Q}_{v}$ is zero on $I_{v} /\left(\left.\operatorname{Im} \mathrm{d} s\right|_{v}\right)$, it follows that $\operatorname{Im} \Pi_{v} \cap\left(I_{v} /\left(\left.\operatorname{Im} \mathrm{d} s\right|_{v}\right)\right)=\{0\}$, and thus

$$
\left.E^{-}\right|_{v} \cap I_{v}=\{0\} .
$$

Condition 3.23, that $\left.t\right|_{v}\left(\left.E^{-}\right|_{v}\right)=\left.t\right|_{v}\left(\left.E\right|_{v}\right)$, is equivalent to the equation $\left.E^{-}\right|_{v}+\operatorname{Ker}\left(\left.t\right|_{v}\right)=\left.E\right|_{v}$, in subspaces of $\left.E\right|_{v}$. As $\operatorname{Im} \Pi_{v}$ is a maximal negative definite subspace for $\operatorname{Re} \tilde{Q}_{v}$ in $\left(\left.\operatorname{Ker} t\right|_{v}\right) /\left(\left.\operatorname{Imd} s\right|_{v}\right)$, and $K_{v} /\left(\left.\operatorname{Im} \mathrm{d} s\right|_{v}\right)$ is the orthogonal to a null subspace $I_{v} /\left(\left.\operatorname{Im} \mathrm{d} s\right|_{v}\right)$ w.r.t. $\operatorname{Re} \tilde{Q}_{v}$, it follows that $\operatorname{Im} \Pi_{v}+K_{v} /\left(\left.\operatorname{Im} \mathrm{d} s\right|_{v}\right)=\left(\left.\operatorname{Ker} t\right|_{v}\right) /\left(\left.\operatorname{Im} \mathrm{d} s\right|_{v}\right)$. Lifting to Ker $\left.t\right|_{v}$ gives $\left[\left.E^{-}\right|_{v} \cap\right.$ $\left.\left(\left.\operatorname{Ker} t\right|_{v}\right)\right]+K_{v}=\left.\operatorname{Ker} t\right|_{v}$. Thus the subspace $\left.E^{-}\right|_{v}+K_{v}$ in $\left.E\right|_{v}$ contains $\left.E^{-}\right|_{v}$ and $\left.\operatorname{Ker} t\right|_{v}$, so as $\left.E^{-}\right|_{v}+\operatorname{Ker}\left(\left.t\right|_{v}\right)=\left.E\right|_{v}$, we see that

$$
\left.E^{-}\right|_{v}+K_{v}=\left.E\right|_{v}
$$

Write $\check{\Pi}_{v}:\left.E^{-}\right|_{v} \cap K_{v} \rightarrow K_{v} / I_{v}$ for the natural projection. It is injective by (5.2). Using (5.2)-(5.3) and the facts that $\operatorname{Im} \Pi_{v}$ has half the dimension of $\left(\left.\operatorname{Ker} t\right|_{v}\right) /\left(\left.\operatorname{Im} \mathrm{d} s\right|_{v}\right)$, and $\operatorname{dim}\left[I_{v} /\left(\left.\operatorname{Im} \mathrm{d} s\right|_{v}\right)\right]+\operatorname{dim}\left[K_{v} /\left(\left.\operatorname{Im} \mathrm{d} s\right|_{v}\right)\right]=$ $\operatorname{dim}\left[\left(\left.\operatorname{Ker} t\right|_{v}\right) /\left(\left.\operatorname{Im} \mathrm{d} s\right|_{v}\right)\right]$ as $I_{v} /\left(\left.\operatorname{Im} \mathrm{d} s\right|_{v}\right), K_{v} /\left(\left.\operatorname{Im} \mathrm{d} s\right|_{v}\right)$ are orthogonal subspaces, by a dimension count we find that $\operatorname{Im} \check{\Pi}_{v}$ has half the total dimension of $K_{v} / I_{v}$. Also, since the quadratic form on $\check{Q}_{v}$ on $K_{v} / I_{v} \cong\left[K_{v} /\left(\left.\operatorname{Im} \mathrm{d} s\right|_{v}\right)\right] /\left[I_{v} /\left(\left.\operatorname{Im} \mathrm{d} s\right|_{v}\right)\right]$ descends from the restriction of $\tilde{Q}_{v}$ to $K_{v} /\left(\left.\operatorname{Im} \mathrm{d} s\right|_{v}\right)$, and $\operatorname{Im} \check{\Pi}_{v}$ descends from 
$\operatorname{Im} \Pi_{v} \cap\left[K_{v} /\left(\left.\operatorname{Imd} s\right|_{v}\right)\right]$, and $\operatorname{Re} \tilde{Q}_{v}$ is negative definite on $\operatorname{Im} \Pi_{v}$, we see that $\operatorname{Re} \check{Q}_{v}$ is negative definite on $\operatorname{Im} \check{\Pi}_{v}$.

As $\left.\left.E^{-}\right|_{v_{i}} \rightarrow E^{-}\right|_{v}$ and $\left.\operatorname{Im} \mathrm{d} s\right|_{v_{i}} \rightarrow I_{v}$ as $i \rightarrow \infty$, we see from (5.2) that

$$
\left.E^{-}\right|_{v_{i}} \cap\left(\left.\operatorname{Im} \mathrm{d} s\right|_{v_{i}}\right)=\{0\} \quad \text { for } i \gg 0 .
$$

Since $\left.\left.E^{-}\right|_{v_{i}} \rightarrow E^{-}\right|_{v}$ and $\left.\operatorname{Ker} t\right|_{v_{i}} \rightarrow K_{v}$ as $i \rightarrow \infty$, we see from (5.3) that $\left.E^{-}\right|_{v_{i}}+\left.\operatorname{Ker} t\right|_{v_{i}}=\left.E\right|_{v_{i}}$ for $i \gg 0$. But this is equivalent to

$$
\left.t\right|_{v_{i}}\left(\left.E^{-}\right|_{v_{i}}\right)=\left.t\right|_{v_{i}}\left(\left.E\right|_{v_{i}}\right) \quad \text { in }\left.F\right|_{v_{i}} \text { for } i \gg 0 .
$$

Using (5.4)-(5.5), the same dimension count as above implies that $\operatorname{Im} \tilde{\Pi}_{v_{i}}$ has half the dimension of $\left(\left.\operatorname{Ker} t\right|_{v_{i}}\right) /\left(\left.\operatorname{Im} \mathrm{d} s\right|_{v_{i}}\right)$ for $i \gg 0$. Under the limit (5.1), we have $\tilde{Q}_{v_{i}} \rightarrow \check{Q}_{v}$ and $\operatorname{Im} \tilde{\Pi}_{v_{i}} \rightarrow \operatorname{Im} \check{\Pi}_{v}$. Thus, as $\operatorname{Re} \check{Q}_{v}$ is negative definite on $\operatorname{Im} \check{\Pi}_{v}$, we see that $\operatorname{Re} \tilde{Q}_{v_{i}}$ is negative definite on $\operatorname{Im} \tilde{\Pi}_{v_{i}}$ for $i \gg 0$. Together with (5.4) -(5.5), this shows that the assumptions on $\left.E^{-}\right|_{v_{i}}$ in (*) hold for $i \gg 0$, which contradicts the choice of sequence $\left(v_{i}\right)_{i=1}^{\infty}$. This proves Theorem 3.7(a).

\subsection{Theorem 3.7(b): extending pairs $\left(U, E^{-}\right)$satisfying $(*)$}

Suppose $\boldsymbol{X}, \omega_{\boldsymbol{X}}^{*}, A^{\bullet}, \boldsymbol{\alpha}, V, E, F, s, t, \psi$ are as in Definition [3.6] and $\left(U, E^{-}\right)$satisfying (*) is as in Definition 3.6, and $C \subseteq V$ is closed with $C \subseteq U$. Our goal is to construct $\left(\tilde{U}, \tilde{E}^{-}\right)$satisfying $(*)$ for $V, E, \ldots$ with $C \cup s^{-1}(0) \subseteq \tilde{U} \subseteq V$, such that $\left.E^{-}\right|_{U^{\prime}}=\left.\tilde{E}^{-}\right|_{U^{\prime}}$ for $U^{\prime}$ an open neighbourhood of $C$ in $U \cap \tilde{\tilde{U}}$.

Using the notation of $93.2, s^{-1}(0)^{\text {alg }}$ is a finite type closed $\mathbb{C}$-subscheme of $V^{\text {alg }}$, and the maps $\left.v \mapsto \operatorname{dim} \operatorname{Ker} \mathrm{d} s\right|_{v},\left.v \mapsto \operatorname{dim} \operatorname{Ker} t\right|_{v}$ are upper semicontinuous, algebraically constructible functions $s^{-1}(0)^{\text {alg }} \rightarrow \mathbb{N}$, noting that $\left.t\right|_{v}$ is independent of choices for $v \in s^{-1}(0)^{\text {alg }}$. Therefore by some standard facts about constructible sets in algebraic geometry, we can choose a stratification of Zariski topological spaces $s^{-1}(0)^{\text {alg }}=\coprod_{a \in A} W_{a}^{\text {alg }}$, where $A$ is a finite indexing set, and $W_{a}^{\text {alg }}$ is a smooth, connected, locally closed $\mathbb{C}$-subscheme of $s^{-1}(0)^{\text {alg }} \subseteq V^{\text {alg }}$ for each $a \in A$, with closure $\bar{W}_{a}^{\text {alg }}$ in $s^{-1}(0)^{\text {alg }}$ a finite union of strata $W_{b}$, such that $\left.v \mapsto \operatorname{dim} \operatorname{Ker} \mathrm{d} s\right|_{v}$ and $\left.v \mapsto \operatorname{dim} \operatorname{Ker} t\right|_{v}$ are both constant functions on $W_{a}^{\text {alg }}$.

Writing $W_{a} \subseteq s^{-1}(0) \subseteq V$ for the set of $\mathbb{C}$-points of $W_{a}^{\text {alg }}$, each $W_{a}$ is a connected, locally closed complex submanifold of $V$ lying in $s^{-1}(0)$, with closure $\bar{W}_{a}$ a finite union of submanifolds $W_{b}$, such that $s^{-1}(0)=\coprod_{a \in A} W_{a}$. On each $W_{a}$, the maps $\left.v \mapsto \operatorname{dim} \operatorname{Ker} \mathrm{d} s\right|_{v}$ and $\left.v \mapsto \operatorname{dim} \operatorname{Ker} t\right|_{v}$ are constant. This implies that Ker $\left.\mathrm{d} s\right|_{W_{a}}$ is a holomorphic vector subbundle of $\left.T V\right|_{W_{a}}$, and $\left.\operatorname{Im} \mathrm{d} s\right|_{W_{a}}$ a holomorphic vector subbundle of $\left.E\right|_{W_{a}}$, and Ker $\left.t\right|_{W_{a}}$ a holomorphic vector subbundle of $\left.E\right|_{W_{a}}$, and $\left.\operatorname{Im} t\right|_{W_{a}}$ a holomorphic vector subbundle of $\left.F\right|_{W_{a}}$. We have $\left.\left.\left.\operatorname{Im} \mathrm{d} s\right|_{W_{a}} \subseteq \operatorname{Ker} t\right|_{W_{a}} \subseteq E\right|_{W_{a}}$ as $t \circ \mathrm{d} s=0$ on $s^{-1}(0)$.

Thus we have a holomorphic vector bundle $\left(\left.\operatorname{Ker} t\right|_{W_{a}}\right) /\left(\left.\operatorname{Im} \mathrm{d} s\right|_{W_{a}}\right)$ over $W_{a}$, whose fibre at $v \in W_{a}$ is identified with $H^{1}\left(\left.\mathbb{T}_{\boldsymbol{X}}\right|_{x}\right)$ for $x=\psi(v)$ by (3.9). As in (2.6) we have a quadratic form $Q_{x}$ on $H^{1}\left(\left.\mathbb{T}_{\boldsymbol{X}}\right|_{x}\right)$, and as in (3.21) $\tilde{Q}_{v}$ is the quadratic form on $\left(\left.\operatorname{Ker} t\right|_{W_{a}}\right) /\left.\left(\left.\operatorname{Im~} \mathrm{d} s\right|_{W_{a}}\right)\right|_{v}$ identified with $Q_{x}$ by (3.9) . One can prove using a representative $\omega_{A}$ • for $\boldsymbol{\alpha}^{*}\left(\omega_{\boldsymbol{X}}^{0}\right)$ that $\tilde{Q}_{v}$ depends holomorphically 
on $v \in W_{a}$. Hence $\tilde{Q}_{v}=\left.\tilde{Q}_{a}\right|_{v}$ for $\tilde{Q}_{a} \in H^{0}\left(S^{2}\left[\left(\left.\operatorname{Ker} t\right|_{W_{a}}\right) /\left(\left.\operatorname{Im~} \mathrm{d} s\right|_{W_{a}}\right)\right]^{*}\right)$, a nondegenerate holomorphic quadratic form on the fibres of $\left(\left.\operatorname{Ker} t\right|_{W_{a}}\right) /\left(\left.\operatorname{Im} d s\right|_{W_{a}}\right)$.

The idea of the proof is to choose $\tilde{E}^{-}$near $W_{a}$ by induction on increasing $\operatorname{dim} W_{a}$, starting with $a \in A$ with $\operatorname{dim} W_{a}=0$, then $\operatorname{dim} W_{a}=1$, and so on. Since $\operatorname{dim}\left(\bar{W}_{a} \backslash W_{a}\right)<\operatorname{dim} W_{a}$, we see that $\bar{W}_{a} \backslash W_{a}$ is a finite union of $W_{b}$ with $\operatorname{dim} W_{b}<\operatorname{dim} W_{a}$, so when we choose $\tilde{E}^{-}$near $W_{a}$ we will already have chosen $\tilde{E}^{-}$near $\bar{W}_{a} \backslash W_{a}$, and the extension over $W_{a}$ should be compatible with this.

Our inductive hypothesis $(\ddagger)_{m}$ for $m=0,1,2, \ldots$ is:

$(\ddagger)_{m}$ For all $a \in A$ with $\operatorname{dim} W_{a} \leqslant m$ we have chosen a pair $\left(\check{U}_{a}, \check{E}_{a}^{-}\right)$satisfying (*) for $V, E, F, s, t, \ldots$ with $W_{a} \subseteq \check{U}_{a} \subseteq V$, such that there is an open neighbourhood $\hat{U}_{a}$ of $C \cap \check{U}_{a}$ in $U \cap \check{U}_{a}$ with $\left.E^{-}\right|_{\hat{U}_{a}}=\left.\check{E}_{a}^{-}\right|_{\hat{U}_{a}}$, and if $b \in A$ with $W_{b} \subseteq \bar{W}_{a} \backslash W_{a}$ (which implies that $\operatorname{dim} W_{b}<\operatorname{dim} W_{a} \leqslant m$, so $\left(\check{U}_{b}, \check{E}_{b}^{-}\right)$is defined), then there is an open neighbourhood $\hat{U}_{a b}$ of $W_{b}$ in $\check{U}_{b}$ such that $\check{E}_{a}^{-}\left|\check{U}_{a} \cap \hat{U}_{a b}=\check{E}_{b}^{-}\right|_{\breve{U}_{a} \cap \hat{U}_{a b}}$.

First consider how to choose $\left(\check{U}_{a}, \check{E}_{a}^{-}\right)$satisfying (*) with $W_{a} \subseteq \check{U}_{a} \subseteq V$ for $a \in A$ with no compatibility conditions, either with $\left(U, E^{-}\right)$near $C$, or with $\left(\check{U}_{b}, \check{E}_{b}^{-}\right)$for $W_{b} \subseteq \bar{W}_{a} \backslash W_{a}$. We can do this as follows:

(i) Choose a real vector subbundle $\dot{E}_{a}$ of $\left(\left.\operatorname{Ker} t\right|_{W_{a}}\right) /\left(\left.\operatorname{Im} \mathrm{d} s\right|_{W_{a}}\right)$, whose real rank is half the real rank of $\left(\left.\operatorname{Ker} t\right|_{W_{a}}\right) /\left(\left.\operatorname{Imd} s\right|_{W_{a}}\right)$, such that $\operatorname{Re} \tilde{Q}_{a}$ is negative definite on $\dot{E}_{a}$.

(ii) Lift $\dot{E}_{a}$ to a real vector subbundle $\ddot{E}_{a}$ of $\left.\operatorname{Ker} t\right|_{W_{a}}$. That is, the projection $\left.\operatorname{Ker} t\right|_{W_{a}} \rightarrow\left(\left.\operatorname{Ker} t\right|_{W_{a}}\right) /\left(\left.\operatorname{Imd} s\right|_{W_{a}}\right)$ induces an isomorphism $\ddot{E}_{a} \rightarrow \dot{E}_{a}$.

(iii) Choose a real vector subbundle $\dddot{E}_{a}$ of $\left.E\right|_{W_{a}}$ with $\left.E\right|_{W_{a}}=\left.\dddot{E}_{a} \oplus \operatorname{Ker} t\right|_{W_{a}}$.

(iv) Set $\left.\check{E}_{a}^{-}\right|_{W_{a}}=\ddot{E}_{a} \oplus \dddot{E}_{a}$. Then $\left.\check{E}_{a}^{-}\right|_{W_{a}}$ is a real vector subbundle of $\left.E\right|_{W_{a}}$, and the assumptions on $\left.\check{E}_{a}^{-}\right|_{v}$ in condition $(*)$ in $\$ 3.3$ hold for all $v \in W_{a}$.

(v) Choose any real vector subbundle $\check{E}_{a}^{-}$of $\left.E\right|_{\breve{U}_{a}}$ on a small open neighbour$\operatorname{hood} \breve{U}_{a}$ of $W_{a}$ in $V$, extending the given $\left.\breve{E}_{a}^{-}\right|_{W_{a}}=\ddot{E}_{a} \oplus \dddot{E}_{a}$ on $W_{a}$.

Observe that by Theorem 3.7(a), proved in $\$ 5.1$, condition $(*)$ holds for $\check{E}_{a}^{-}$on an open neighbourhood of $W_{a}$. So by making $\check{U}_{a}$ smaller, we can suppose $\left(\check{U}_{a}, \check{E}_{a}^{-}\right)$satisfies $(*)$.

All of these are possible. Any $\left(\check{U}_{a}, \check{E}_{a}^{-}\right)$satisfying $(*)$ with $W_{a} \subseteq \check{U}_{a} \subseteq V$ arises from steps (i)-(v) (though $\dddot{E}_{a}$ in (iii) is not uniquely determined by $\check{E}_{a}^{-}$). Furthermore (taking germs in $(\mathrm{v})$ ), the space of choices in each step is contractible.

Now suppose $m=0,1, \ldots$, and $(\ddagger)_{m-1}$ holds if $m>0$, and $a \in A$ with $\operatorname{dim} W_{a}=m$. To choose $\left(\check{U}_{a}, \check{E}_{a}^{-}\right)$with the compatibility conditions required in $(\ddagger)_{m}$, we follow (i)-(v), but modified as follows. In step (i), we choose $\dot{E}_{a}$ with

$$
\left.\dot{E}_{a}\right|_{W_{a} \cap \hat{U}_{a}}=\left[\left(\left.\left(E^{-} \cap \operatorname{Ker} t\right)\right|_{W_{a} \cap \hat{U}_{a}}\right)+\left(\left.\operatorname{Im} \mathrm{d} s\right|_{W_{a} \cap \hat{U}_{a}}\right)\right] /\left(\left.\operatorname{Im} \mathrm{d} s\right|_{W_{a} \cap \hat{U}_{a}}\right),
$$

for some small open neighbourhood $\hat{U}_{a}$ of $C \cap W_{a}$ in $U$, and if $b \in A$ with $W_{b} \subseteq \bar{W}_{a} \backslash W_{a}$ then

$$
\left.\dot{E}_{a}\right|_{W_{a} \cap \check{U}_{a b}}=\left[\left(\left(\left.\check{E}_{b}^{-} \cap \operatorname{Ker} t\right|_{W_{a} \cap \hat{U}_{a b}}\right)\right)+\left(\left.\operatorname{Im} \mathrm{d} s\right|_{W_{a} \cap \hat{U}_{a b}}\right)\right] /\left(\left.\operatorname{Imd} s\right|_{W_{a} \cap \hat{U}_{a b}}\right),
$$


for some small open neighbourhood $\hat{U}_{a b}$ of $W_{b}$ in $\check{U}_{b}$.

To see this is possible, first note that the first part of $(\ddagger)_{m-1}$ with $b$ in place of $a$ implies that equations (5.6) and (5.7) are compatible, that is they prescribe the same value for $\dot{E}_{a}$ on $W_{a} \cap \hat{U}_{a} \cap \hat{U}_{a b}$, provided the open neighbourhoods $\hat{U}_{a}, \hat{U}_{a b}$ are small enough. Also given distinct $b, b^{\prime} \in A$ with $W_{b}, W_{b^{\prime}} \subseteq \bar{W}_{a} \backslash W_{a}$, either (a) $W_{b^{\prime}} \subseteq \bar{W}_{b} \backslash W_{b}$, or (b) $W_{b} \subseteq \bar{W}_{b^{\prime}} \backslash W_{b^{\prime}}$, or (c) $W_{b} \cap \bar{W}_{b^{\prime}}=\bar{W}_{b} \cap W_{b^{\prime}}=\emptyset$. In cases (a),(b) we can use the second part of $(\ddagger)_{m-1}$ to show that (5.7) for $b, b^{\prime}$ are compatible provided $\hat{U}_{a b}, \hat{U}_{a b^{\prime}}$ are small enough, and in case (c) we can choose $\hat{U}_{a b}, \hat{U}_{a b^{\prime}}$ with $\hat{U}_{a b} \cap \hat{U}_{a b^{\prime}}=\emptyset$, so compatibility is trivial.

Thus, if $\hat{U}_{a}$ and $\hat{U}_{a b}$ for all $b$ are small enough then (5.6) and (5.7) for all $b$ are compatible, and can be combined into a single equation prescribing $\dot{E}_{a}$ on $\breve{W}_{a}:=W_{a} \cap\left(\hat{U}_{a} \cup \bigcup_{b} \hat{U}_{a b}\right)$. We then have to extend $\dot{E}_{a}$ from $\check{W}_{a}$ to $W_{a}$, satisfying the required conditions. This may not be possible: if we have chosen $E^{-}$or $\check{E}_{b}^{-}$badly near the 'edge' of $\check{W}_{a}$ in $W_{a}$, then the prescribed values of $\dot{E}_{a}$ may not extend continuously to the closure $\bar{W}_{a}$ of $\check{W}_{a}$ in $W_{a}$. However, we can deal with this problem by shrinking all the $\hat{U}_{a}, \hat{U}_{a b}$, such that the closure $\check{W}_{a}$ of the new $\check{W}_{a}$ lies inside the old $\check{W}_{a}$. Then it is guaranteed that the prescribed value of $\dot{E}_{a}$ on $\check{W}_{a}$ extends smoothly to an open neighbourhood of $\overline{\breve{W}_{a}}$ in $W_{a}$, so we can choose $\dot{E}_{a}$ on $W_{a}$ satisfying all the required conditions (5.6)-(5.7).

In a similar way, for each of steps (ii)-(v) we can show that making the open neighbourhoods $\hat{U}_{a}, \hat{U}_{a b}$ smaller if necessary, we can make choices consistent with the compatibility conditions on $\left(\check{U}_{a}, \check{E}_{a}^{-}\right)$in $(\ddagger)_{m}$. So by induction, $(\ddagger)_{m}$ holds for all $m=0,1, \ldots$ Fix data $\left(\check{U}_{a}, \check{E}_{a}^{-}\right), \hat{U}_{a}, \hat{U}_{a b}$ satisfying $(\ddagger)_{m}$ for $m=\operatorname{dim} V$.

Next, choose open neighbourhoods $U^{\prime}$ of $C$ in $U \subseteq V$ and $\tilde{U}_{a}$ of $W_{a}$ in $\check{U}_{a}$ for each $a \in A$, such that $U^{\prime} \cap \tilde{U}_{a} \subseteq \hat{U}_{a}$ for $a \in A$, and $\tilde{U}_{a} \cap \tilde{U}_{b} \subseteq \hat{U}_{a b}$ if $a, b \in A$ with $W_{b} \subseteq \bar{W}_{a} \backslash W_{a}$, and $\tilde{U}_{a} \cap \tilde{U}_{b}=\emptyset$ if $a, b \in A$ with $\bar{W}_{a} \cap W_{b}=W_{a} \cap \bar{W}_{b}=\emptyset$. This is possible provided $U^{\prime}$ and $\tilde{U}_{a}$ for $a \in A$ are all small enough.

Define $\tilde{U}=U^{\prime} \cup \bigcup_{a \in A} \tilde{U}_{a}$, which is an open neighbourhood of $C \cup \bigcup_{a \in A} W_{a}=$ $C \cup s^{-1}(0)$ in $V$. Define a vector subbundle $\tilde{E}^{-}$of $\left.E\right|_{\tilde{U}}$ by $\left.\tilde{E}^{-}\right|_{U^{\prime}}=\left.E^{-}\right|_{U^{\prime}}$ and $\left.\tilde{E}^{-}\right|_{\tilde{U}_{a}}=\left.\tilde{E}_{a}^{-}\right|_{\tilde{U}_{a}}$ for $a \in A$. These values are consistent on overlaps $U^{\prime} \cap \tilde{U}_{a}$ and $\tilde{U}_{a} \cap \tilde{U}_{b}$ by construction, so $\tilde{E}^{-}$is well-defined. Also $\left(\tilde{U}, \tilde{E}^{-}\right)$satisfies $(*)$, since $\left(U, E^{-}\right)$and the $\left(\check{U}_{a}, \check{E}_{a}^{-}\right)$do, and $U^{\prime}$ is an open neighbourhood of $C$ in $U \cap \tilde{U}$ with $\left.E^{-}\right|_{U^{\prime}}=\left.\tilde{E}^{-}\right|_{U^{\prime}}$ by definition. This proves Theorem $3.7(\mathrm{~b})$.

\subsection{Theorem 3.7(c): $s^{-1}(0)=\left(s^{+}\right)^{-1}(0)$ locally in $U$}

In 33.4 we explained how to pullback pairs $\left(U_{K}, E_{K}^{-}\right)$satisfying $(*)$ along a quasi-free $\Phi_{J K}: A_{K}^{\bullet} \rightarrow A_{J}^{\bullet}$. We can also pushforward $\left(U_{J}, E_{J}^{-}\right)$along $\Phi_{J K}$.

Definition 5.1. Let $\boldsymbol{X}, \omega_{\boldsymbol{X}}^{*}, n, \Phi_{J K}: A_{K}^{\bullet} \rightarrow A_{J}^{\bullet}$ and $V_{J}, E_{J}, \ldots, \chi_{J K}, \xi_{J K}$ be as in Definition 3.10, and suppose $\left(U_{J}, E_{J}^{-}\right)$satisfies $(*)$ for $A_{J}^{\bullet}$. Our goal is to construct $\left(U_{K}, E_{K}^{-}\right)$satisfying $(*)$ for $A_{K}^{\bullet}$, with $\psi_{J}\left(s_{J}^{-1}(0) \cap U_{J}\right)=\psi_{K}\left(s_{K}^{-1}(0) \cap\right.$ $\left.U_{K}\right) \subseteq X_{\text {an }}$, and if $\left(U_{J}, E_{J}^{-}\right),\left(U_{K}, E_{K}^{-}\right)$also satisfy $(\dagger)$, a coordinate change of Kuranishi neighbourhoods, as in 92.5 .

$$
\left(U_{K}, \theta_{K J}, \eta_{K J}\right):\left(U_{K}, E_{K}^{+}, s_{K}^{+}, \psi_{K}^{+}\right) \longrightarrow\left(U_{J}, E_{J}^{+}, s_{J}^{+}, \psi_{J}^{+}\right) .
$$


Let $v_{J} \in s_{J}^{-1}(0) \cap U_{J}$ with $\phi_{J K}\left(v_{J}\right)=v_{K} \in s_{K}^{-1}(0) \subseteq V_{K}$ and $\psi_{J}\left(v_{J}\right)=$ $\psi_{K}\left(v_{K}\right)=x \in X_{\mathrm{an}}$. We claim that we can choose splittings of real vector spaces

$$
\begin{array}{ll}
T_{v_{J}} V_{J}=\tilde{T}_{v_{J}} V_{J} \oplus T_{v_{J}}^{\prime} V_{J}, & \left.E_{J}\right|_{v_{J}}=\left.\left.\left.\tilde{E}_{J}\right|_{v_{J}} \oplus E_{J}^{\prime}\right|_{v_{J}} \oplus E_{J}^{\prime \prime}\right|_{v_{J}}, \\
\left.E_{J}^{-}\right|_{v_{J}}=\left.\left.\tilde{E}_{J}^{-}\right|_{v_{J}} \oplus \tilde{E}_{J}^{\prime \prime}\right|_{v_{J}}, & \left.F_{J}\right|_{v_{J}}=\left.\left.\left.\tilde{F}_{J}\right|_{v_{J}} \oplus F_{J}^{\prime \prime}\right|_{v_{J}} \oplus F_{J}^{\prime \prime \prime}\right|_{v_{J}},
\end{array}
$$

fitting into a commutative diagram of the form

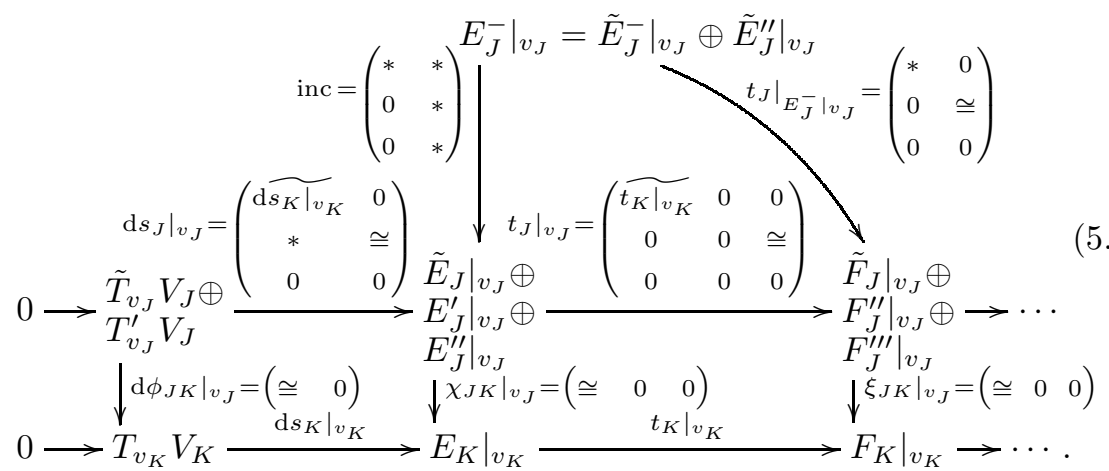

To prove this, note that the rows of (5.10) are $\left.\mathbb{T}_{\mathbf{S p e c} A_{J}^{\bullet}}\right|_{v_{J}},\left.\mathbb{T}_{\mathbf{S p e c} A_{K}^{\bullet}}\right|_{v_{K}}$, and are complexes, and the lower columns are induced by $\Phi_{J K}$, are surjective as $\Phi_{J K}$ is quasi-free, and induce isomorphisms on cohomology as in $\$ 3.2$. Then:

(i) Define $T_{v_{J}}^{\prime} V_{J}=\left.\operatorname{Ker} \mathrm{d} \phi_{J K}\right|_{v_{J}}$.

(ii) Choose arbitrary $\tilde{T}_{v_{J}} V_{J}$ with $T_{v_{J}} V_{J} \cong \tilde{T}_{v_{J}} V_{J} \oplus T_{v_{J}}^{\prime} V_{J}$. Then $\tilde{T}_{v_{J}} V_{J} \cong$ $T_{v_{K}} V_{K}$ as $\mathrm{d} \phi_{J K}$ is surjective.

(iii) Define $\left.E_{J}^{\prime}\right|_{v_{J}}=\left.\mathrm{d} s_{J}\right|_{v_{J}}\left[T_{v_{J}}^{\prime} V_{J}\right]$. Then $\left.E_{J}^{\prime}\right|_{v_{J}} \cong T_{v_{J}}^{\prime} V_{J}$ as the columns of (5.10) are isomorphisms in cohomology, and $\left.E_{J}^{\prime}\right|_{v_{J}} \subseteq \operatorname{Ker}\left(\left.\chi_{J K}\right|_{v_{J}}\right)$ as the left hand square of (5.10) commutes.

(iv) Choose $\left.E_{J}^{\prime \prime}\right|_{v_{J}}$ with $\operatorname{Ker}\left(\left.\chi_{J K}\right|_{v_{J}}\right)=\left.\left.E_{J}^{\prime}\right|_{v_{J}} \oplus E_{J}^{\prime \prime}\right|_{v_{J}}$.

(v) Since the columns of (5.10) are isomorphisms on cohomology, $\left.t_{J}\right|_{v_{J}}$ is injective on $\left.E_{J}^{\prime \prime}\right|_{v_{J}}$. Define $\left.F_{J}^{\prime \prime}\right|_{v_{J}}=\left.t_{J}\right|_{v_{J}}\left[\left.E_{J}^{\prime \prime}\right|_{v_{J}}\right]$. Then $\left.\left.F_{J}^{\prime \prime}\right|_{v_{J}} \cong E_{J}^{\prime \prime}\right|_{v_{J}}$. Also $\left.\left.F_{J}^{\prime \prime}\right|_{v_{J}} \subseteq \operatorname{Ker} \xi_{J K}\right|_{v_{J}}$, as the right hand square of (5.10) commutes.

(vi) Choose $\left.F_{J}^{\prime \prime \prime}\right|_{v_{J}}$ with $\left.\operatorname{Ker} \xi_{J K}\right|_{v_{J}}=\left.\left.F_{J}^{\prime \prime}\right|_{v_{J}} \oplus F_{J}^{\prime \prime \prime}\right|_{v_{J}}$.

(vii) Since the columns of (5.10) are isomorphisms on cohomology, we have

$$
\begin{aligned}
\left.F_{J}^{\prime \prime}\right|_{v_{J}} & =\left.t_{J}\right|_{v_{J}}\left[\left.\left.E_{J}^{\prime}\right|_{v_{J}} \oplus E_{J}^{\prime \prime}\right|_{v_{J}}\right]=\left.t_{J}\right|_{v_{J}}\left[\left.\operatorname{Ker} \chi_{J K}\right|_{v_{J}}\right] \\
& =\left.\left.\operatorname{Ker} \xi_{J K}\right|_{v_{J}} \cap \operatorname{Im} t_{J}\right|_{v_{J}}=\left.\left(\left.\left.F_{J}^{\prime \prime}\right|_{v_{J}} \oplus F_{J}^{\prime \prime \prime}\right|_{v_{J}}\right) \cap \operatorname{Im} t_{J}\right|_{v_{J}} .
\end{aligned}
$$

Thus we may choose $\left.\tilde{F}_{J}\right|_{v_{J}}$ with $\left.F_{J}\right|_{v_{J}}=\left.\left.\left.\tilde{F}_{J}\right|_{v_{J}} \oplus F_{J}^{\prime \prime}\right|_{v_{J}} \oplus F_{J}^{\prime \prime \prime}\right|_{v_{J}}$ and $\left.\left.\left.\operatorname{Im} t_{J}\right|_{v_{J}} \subseteq \tilde{F}_{J}\right|_{v_{J}} \oplus F_{J}^{\prime \prime}\right|_{v_{J}}$. So the third row of $\left.t_{J}\right|_{v_{J}}$ in (15.10) is zero. Also $\left.\left.\tilde{F}_{J}\right|_{v_{J}} \cong F_{K}\right|_{v_{K}}$ by (vi) as $\xi_{J K}$ is surjective. 
(viii) Set $\left.\tilde{E}_{J}^{-}\right|_{v_{J}}=\left.\left.E_{J}^{-}\right|_{v_{J}} \cap t_{J}\right|_{v_{J}} ^{-1}\left(\left.\tilde{F}_{J}\right|_{v_{J}}\right)$. We claim $\left.\chi_{J K}\right|_{v_{J}}$ is injective on $\left.\tilde{E}_{J}^{-}\right|_{v_{J}}$. To see this, note that we have an exact sequence

$$
\left.\left.\left.\left.\left.0 \longrightarrow E_{J}^{-}\right|_{v_{J}} \cap \operatorname{Ker} t_{J}\right|_{v_{J}} \longrightarrow \tilde{E}_{J}^{-}\right|_{v_{J}} \longrightarrow t_{J}\right|_{v_{J}}\left[\left.E_{J}^{-}\right|_{v_{J}}\right] \cap \tilde{F}_{J}\right|_{v_{J}} \longrightarrow 0
$$

as $\left.\left.\operatorname{Ker} t_{J}\right|_{v_{J}} \subseteq t_{J}\right|_{v_{J}} ^{-1}\left(\left.\tilde{F}_{J}\right|_{v_{J}}\right)$. The last part of $(*)$ implies that $\left.\chi_{J K}\right|_{v_{J}}$ maps $\left.\left.E_{J}^{-}\right|_{v_{J}} \cap \operatorname{Ker} t_{J}\right|_{v_{J}}$ injectively into $\left.\operatorname{Ker} t_{K}\right|_{v_{K}}$. Also $\left.\xi_{J K}\right|_{v_{J}}$ is injective on $\left.\tilde{F}_{J}\right|_{v_{J}}$, and the right square of (5.10) commutes, so the claim follows.

(ix) Choose $\left.\left.\tilde{E}_{J}\right|_{v_{J}} \subseteq E_{J}\right|_{v_{J}}$ such that $\left.\left.\tilde{E}_{J}^{-}\right|_{v_{J}} \subseteq \tilde{E}_{J}\right|_{v_{J}}$ and $\left.E_{J}\right|_{v_{J}}=\left.\tilde{E}_{J}\right|_{v_{J}} \oplus$ $\operatorname{Ker}\left(\left.\chi_{J K}\right|_{v_{J}}\right)=\left.\left.\left.\tilde{E}_{J}\right|_{v_{J}} \oplus E_{J}^{\prime}\right|_{v_{J}} \oplus E_{J}^{\prime \prime}\right|_{v_{J}}$ by (iv) and $\left.\left.t_{J}\right|_{v_{J}}\left[\left.\tilde{E}_{J}\right|_{v_{J}}\right] \subseteq \tilde{F}_{J}\right|_{v_{J}}$. This is possible as $\left.\chi_{J K}\right|_{v_{J}}$ is injective on $\left.\tilde{E}_{J}^{-}\right|_{v_{J}}$, and using (v),(vii) and (viii). Then $\left.\left.\tilde{E}_{J}\right|_{v_{J}} \cong E_{K}\right|_{v_{K}}$ as $\chi_{J K}$ is surjective.

(x) Choose $\left.\tilde{E}_{J}^{\prime \prime}\right|_{v_{J}}$ such that $\left.E_{J}^{-}\right|_{v_{J}}=\left.\left.\tilde{E}_{J}^{-}\right|_{v_{J}} \oplus \tilde{E}_{J}^{\prime \prime}\right|_{v_{J}}$ and $\left.\left.t_{J}\right|_{v_{J}}\left[\left.\tilde{E}_{J}^{\prime \prime}\right|_{v_{J}}\right] \subseteq F_{J}^{\prime \prime}\right|_{v_{J}}$. This is possible by (viii) and $\left.\left.\left.\operatorname{Im} t_{J}\right|_{v_{J}} \subseteq \tilde{F}_{J}\right|_{v_{J}} \oplus F_{J}^{\prime \prime}\right|_{v_{J}}$.

As $\left.t_{J}\right|_{v_{J}}\left(\left.E_{J}^{-}\right|_{v_{J}}\right)=\left.t_{J}\right|_{v_{J}}\left(\left.E_{J}\right|_{v_{J}}\right)$ by (3.23) and $\left.F_{J}^{\prime \prime}\right|_{v_{J}}=\left.t_{J}\right|_{v_{J}}\left[\left.E_{J}^{\prime \prime}\right|_{v_{J}}\right]$, we see that $\left.t_{J}\right|_{v_{J}}\left[\left.\tilde{E}_{J}^{\prime \prime}\right|_{v_{J}}\right]=\left.F_{J}^{\prime \prime}\right|_{v_{J}}$. Also $\left.t_{J}\right|_{v_{J}}:\left.\left.\tilde{E}_{J}^{\prime \prime}\right|_{v_{J}} \rightarrow F_{J}^{\prime \prime}\right|_{v_{J}}$ is injective, as $\left.\left.\operatorname{Ker} t_{J}\right|_{\left.E_{J}^{-}\right|_{v_{J}}} \subseteq E_{J}^{-}\right|_{v_{J}}$ by (viii). Hence $\left.\left.\tilde{E}_{J}^{\prime \prime}\right|_{v_{J}} \cong F_{J}^{\prime \prime}\right|_{v_{J}}$.

We can do all this, not just at one $v_{J} \in s_{J}^{-1}(0) \cap U_{J}$, but in an open neighbourhood $U_{J}^{\prime}$ of $s_{J}^{-1}(0) \cap U_{J}$ in $U_{J}$. That is, we can choose $U_{J}^{\prime}$, and splittings

$$
\begin{aligned}
\left.T V_{J}\right|_{U_{J}^{\prime}} & =\tilde{T} V_{J} \oplus T^{\prime} V_{J}, & \left.E_{J}\right|_{U_{J}^{\prime}} & =\left.\tilde{E}_{J} \oplus E_{J}^{\prime} \oplus E_{J}^{\prime \prime}\right|_{v_{J}}, \\
\left.E_{J}^{-}\right|_{U_{J}^{\prime}} & =\tilde{E}_{J}^{-} \oplus \tilde{E}_{J}^{\prime \prime}, & \left.F_{J}\right|_{U_{J}^{\prime}} & =\tilde{F}_{J} \oplus F_{J}^{\prime \prime} \oplus F_{J}^{\prime \prime \prime},
\end{aligned}
$$

with $\tilde{E}_{J}^{-} \subseteq \tilde{E}_{J}$, such that (5.10) holds at each $v_{J} \in s_{J}^{-1}(0) \cap U_{J}$. To see this, note that the argument above can be carried out on $s_{J}^{-1}(0) \cap U_{J}$ regarded as a $C^{\infty}$-subscheme of $U_{J}$, in the sense of $C^{\infty}$-algebraic geometry in [17, and the splittings (5.11) with $\tilde{E}_{J}^{-} \subseteq \tilde{E}_{J}$ can then be extended from $s_{J}^{-1}(0) \cap U_{J}$ to an open neighbourhood $U_{J}^{\prime}$. Making $U_{J}^{\prime}$ smaller, we can suppose the component of $\chi_{J K}$ mapping $\left.\tilde{E}_{J} \rightarrow \phi_{J K}\right|_{U_{J}^{\prime}} ^{*}\left(E_{K}\right)$ is an isomorphism. We can also choose the splittings so that away from $s_{J}^{-1}(0) \cap U_{J},\left.t_{J}\right|_{U_{J}^{\prime}}$ has the form

$$
\left.t_{J}\right|_{U_{J}^{\prime}}=\left(\begin{array}{ccc}
* & * & 0 \\
* & * & \cong \\
* & * & 0
\end{array}\right):\left.\tilde{E}_{J}\right|_{v_{J}} \oplus E_{J}^{\prime} \oplus E_{J}^{\prime \prime} \longrightarrow \tilde{F}_{J} \oplus F_{J}^{\prime \prime} \oplus F_{J}^{\prime \prime \prime} .
$$

Write $\left.s_{J}\right|_{U_{J}^{\prime}}=\tilde{s}_{J} \oplus s_{J}^{\prime} \oplus s_{J}^{\prime \prime}$, for $\tilde{s}_{J} \in C^{\infty}\left(\tilde{E}_{J}\right), s_{J}^{\prime} \in C^{\infty}\left(E_{J}^{\prime}\right)$ and $s_{J}^{\prime \prime} \in$ $C^{\infty}\left(E_{J}^{\prime \prime}\right)$. Then (5.12) and $t_{J} \circ s_{J}=0$ imply that $s_{J}^{\prime \prime}=0$. From (5.10) we see that at each $v_{J} \in s_{J}^{-1}(0) \cap U_{J},\left.\mathrm{~d} s_{J}^{\prime}\right|_{v_{J}}:\left.T_{v_{J}} V_{J} \rightarrow E_{J}^{\prime}\right|_{v_{J}}$ is surjective, and $\left.\mathrm{d} \phi_{J K}\right|_{v_{J}}: \operatorname{Ker}\left(\left.\mathrm{d} s_{J}^{\prime}\right|_{v_{J}}\right) \rightarrow T_{v_{K}} V_{K}$ is an isomorphism. Hence $s_{J}^{\prime}$ is transverse near $v_{J}$, so that $\left(s_{J}^{\prime}\right)^{-1}(0)$ is an embedded submanifold of $V_{J}$ near $v_{J}$ with tangent space $\operatorname{Ker}\left(\left.\mathrm{d} s_{J}^{\prime}\right|_{v_{J}}\right)$ at $v_{J}$, and $\left.\phi_{J K}\right|_{\left(s_{J}^{\prime}\right)^{-1}(0)}:\left(s_{J}^{\prime}\right)^{-1}(0) \rightarrow V_{K}$ is a local diffeomorphism near $v_{J}$. Thus, making $U_{J}^{\prime}$ smaller, we can suppose that $s_{J}^{\prime}$ is transverse on $U_{J}^{\prime}$, so that $\left(s_{J}^{\prime}\right)^{-1}(0)$ is an embedded submanifold 
of $U_{J}^{\prime}$, and $\left.\phi_{J K}\right|_{\left(s_{J}^{\prime \prime}\right)^{-1}(0)}:\left(s_{J}^{\prime}\right)^{-1}(0) \rightarrow V_{K}$ is a local diffeomorphism. But $\phi_{J K}$ is injective on $s_{J}^{-1}(0) \cap U_{J}$, so making $U_{J}^{\prime}$ smaller, we can also suppose that $\left.\phi_{J K}\right|_{\left(s_{J}^{\prime}\right)^{-1}(0)}$ is a diffeomorphism with an open set $U_{K}$ in $V_{K}$, with inverse $\theta_{K J}: U_{K} \stackrel{\cong}{\longrightarrow}\left(s_{J}^{\prime}\right)^{-1}(0) \subseteq U_{J}^{\prime} \subseteq U_{J}$.

We now have a vector bundle $\theta_{K J}^{*}\left(E_{J}\right)$ over $U_{K}$, and vector subbundles $\theta_{K J}^{*}\left(\tilde{E}_{J}, E_{J}^{\prime}, E_{J}^{\prime \prime}, E_{J}^{-}, \tilde{E}_{J}^{-}, \tilde{E}_{J}^{\prime \prime}\right)$ with $\theta_{K J}^{*}\left(E_{J}\right)=\theta_{K J}^{*}\left(\tilde{E}_{J}\right) \oplus \theta_{K J}^{*}\left(E_{J}^{\prime}\right) \oplus \theta_{K J}^{*}\left(E_{J}^{\prime \prime}\right)$, $\theta_{K J}^{*}\left(E_{J}^{-}\right)=\theta_{K J}^{*}\left(\tilde{E}_{J}^{-}\right) \oplus \theta_{K J}^{*}\left(\tilde{E}_{J}^{\prime \prime}\right)$ and $\theta_{K J}^{*}\left(\tilde{E}_{J}^{-}\right) \subseteq \theta_{K J}^{*}\left(\tilde{E}_{J}\right)$. Since $\phi_{J K} \circ \theta_{K J}=$ $\operatorname{id}_{U_{K}}$, pulling back $\chi_{J K}: E_{J} \rightarrow \phi_{J K}^{*}\left(E_{K}\right)$ by $\theta_{K J}$ gives a surjective vector bundle morphism $\theta_{K J}^{*}\left(\chi_{J K}\right):\left.\theta_{K J}^{*}\left(E_{J}\right) \rightarrow E_{K}\right|_{U_{K}}$, where $\theta_{K J}^{*}\left(\chi_{J K}\right)$ restricts to an isomorphism $\theta_{K J}^{*}\left(\tilde{E}_{J}\right) \rightarrow E_{K}$. We also have a section $\theta_{K J}^{*}\left(s_{J}\right)$ of $\theta_{K J}^{*}\left(E_{J}\right)$, whose components in $\theta_{K J}^{*}\left(\tilde{E}_{J}\right), \theta_{K J}^{*}\left(E_{J}^{\prime}\right), \theta_{K J}^{*}\left(E_{J}^{\prime \prime}\right)$ are $\theta_{K J}^{*}\left(\tilde{s}_{J}\right), 0,0$. Applying $\theta_{K J}^{*}$ to (3.14) and using $E_{J}^{\prime \prime} \subseteq \operatorname{Ker} \chi_{J K}$ shows that

$$
\theta_{K J}^{*}\left(\chi_{J K}\right)\left[\theta_{K J}^{*}\left(s_{J}\right)\right]=\theta_{K J}^{*}\left(\chi_{J K}\right)\left[\theta_{K J}^{*}\left(\tilde{s}_{J}\right)\right]=\left.s_{K}\right|_{U_{K}} .
$$

Define a vector subbundle $\left.E_{K}^{-} \subseteq E_{K}\right|_{U_{K}}$ by $E_{K}^{-}=\theta_{K J}^{*}\left(\chi_{J K}\right)\left[\theta_{K J}^{*}\left(\tilde{E}_{J}^{-}\right)\right]$. This is valid as $\theta_{K J}^{*}\left(\tilde{E}_{J}^{-}\right) \subseteq \theta_{K J}^{*}\left(\tilde{E}_{J}\right)$, and $\theta_{K J}^{*}\left(\chi_{J K}\right)$ is an isomorphism on $\theta_{K J}^{*}\left(\tilde{E}_{J}\right)$. We claim that $\left(U_{K}, E_{K}^{-}\right)$satisfies condition $(*)$. To see this, let $v_{K} \in s_{K}^{-1}(0) \cap U_{K}$, and set $v_{J}=\theta_{K J}\left(v_{K}\right)$. Then $v_{J} \in s_{J}^{-1}(0) \cap U_{J}^{\prime}$ with $\phi_{J K}\left(v_{J}\right)=v_{K}$, so (5.9) (5.10) hold, with the columns of (5.10) isomorphisms on cohomology. From this and $(*)$ for $\left(U_{J}, E_{J}^{-}\right)$at $v_{J}$, we can deduce $(*)$ for $\left(U_{K}, E_{K}^{-}\right)$at $v_{K}$.

Writing $E_{J}^{+}=\left.E_{J}\right|_{U_{J}} / E_{J}^{-}, s_{J}^{+}=s_{J}+E_{J}^{-} \in C^{\infty}\left(E_{J}^{+}\right)$, and similarly for $E_{K}^{+}, s_{K}^{+}$, define a vector bundle morphism $\eta_{K J}: E_{K}^{+} \rightarrow \theta_{K J}^{*}\left(E_{J}^{+}\right)$by

$$
\eta_{K J}: e_{K}+\left.E_{K}^{-} \longmapsto \theta_{K J}^{*}\left(\chi_{J K}\right)\right|_{\theta_{K J}^{*}\left(\tilde{E}_{J}\right)} ^{-1}\left[e_{K}\right]+\theta_{K J}^{*}\left(E_{J}^{-}\right) .
$$

This is well-defined as $\left.\theta_{K J}^{*}\left(\chi_{J K}\right)\right|_{\theta_{K J}^{*}\left(\tilde{E}_{J}\right)}: \theta_{K J}^{*}\left(\tilde{E}_{J}\right) \rightarrow E_{K}$ is an isomorphism, with inverse $\left.\theta_{K J}^{*}\left(\chi_{J K}\right)\right|_{\theta_{K J}^{*}\left(\tilde{E}_{J}\right)} ^{-1}: E_{K} \rightarrow \theta_{K J}^{*}\left(\tilde{E}_{J}\right)$, which maps $E_{K}^{-} \rightarrow \theta_{K J}^{*}\left(\tilde{E}_{J}^{-}\right)$ $\subseteq \theta_{K J}^{*}\left(E_{J}^{-}\right)$by definition of $E_{K}^{-}$. Also (5.13) implies that $\eta_{K J}\left(s_{K}^{+}\right)=\theta_{K J}^{*}\left(s_{J}^{+}\right)$. Using (5.10) we can also show that the analogue of (2.8) for $\theta_{K J}, \eta_{K J}$ at $v_{K}$ is exact. Therefore, if $\left(U_{J}, E_{J}^{-}\right),\left(U_{K}, E_{K}^{-}\right)$also satisfy $(\dagger)$, then $\left(U_{K}, \theta_{K J}, \eta_{K J}\right)$ in (5.8) is a coordinate change. This completes Definition 5.1

We now prove Theorem 3.7 (c). Suppose $\boldsymbol{X}, \omega_{\boldsymbol{X}}^{*}, A^{\bullet}, \boldsymbol{\alpha}, V, E, F, s, t, \psi$ and $\left(U, E^{-}\right)$satisfying $(*)$ are as in Definition 3.6. Then $\boldsymbol{X}^{\prime}:=\boldsymbol{\alpha}\left(\operatorname{Spec} A^{\bullet}\right) \subseteq \boldsymbol{X}$ is an affine derived $\mathbb{C}$-subscheme of $\boldsymbol{X}$. Let $v \in s^{-1}(0) \cap U$, and set $x=$ $\psi(v) \in X_{\text {an }}$. Write $\left(A_{1}^{\bullet}, \boldsymbol{\alpha}_{1}\right)=\left(A^{\bullet}, \boldsymbol{\alpha}\right), V_{1}=V, E_{1}=E, v_{1}=v$ and so on. Applying Theorem 2.10 to $\left(\boldsymbol{X}^{\prime},\left.\omega_{\boldsymbol{X}}^{*}\right|_{\boldsymbol{X}^{\prime}}\right)$ at $x$ gives a pair $\left(A_{2}^{\bullet}, \omega_{A_{\dot{2}}}\right)$ in -2Darboux form and a Zariski open inclusion $\boldsymbol{\alpha}_{2}$ : Spec $A_{2}^{\bullet} \hookrightarrow \boldsymbol{X}^{\prime} \subseteq \boldsymbol{X}^{2}$ which is minimal at $x \in \operatorname{Im} \boldsymbol{\alpha}_{2}$ with $\boldsymbol{\alpha}_{2}^{*}\left(\omega_{\boldsymbol{X}}^{*}\right) \simeq \omega_{A_{2}}$. Section 3.2 applied to $A_{2}^{\bullet}, \boldsymbol{\alpha}_{2}$ gives $V_{2}, E_{2}, s_{2}, \ldots$ Set $v_{2}=\psi_{2}^{-1}(x) \in s_{2}^{-1}(0) \subseteq V_{2}$.

Applying Theorem 3.1 to the derived $\mathbb{C}$-scheme $\boldsymbol{X}^{\prime}$ with $I=\{1,2\}$ and initial data $\left\{\left(A_{1}^{\bullet}, \boldsymbol{\alpha}_{1}\right),\left(A_{2}^{\bullet}, \boldsymbol{\alpha}_{2}\right)\right\}$ gives $\left(A_{12}^{\bullet}, \boldsymbol{\alpha}_{12}\right)$ with image $\operatorname{Im} \boldsymbol{\alpha}_{12}=\operatorname{Im} \boldsymbol{\alpha}_{1} \cap$ $\operatorname{Im} \boldsymbol{\alpha}_{2}$ and quasi-free morphisms $\Phi_{12,1}: A_{1}^{\bullet} \rightarrow A_{12}^{\bullet}, \Phi_{12,2}: A_{2}^{\bullet} \rightarrow A_{12}^{\bullet}$ such that (3.3) homotopy commutes in $\mathbf{d} \mathbf{S} \mathbf{c h}_{\mathbb{C}}$. Section 3.2 applied to $A_{12}^{\bullet}$ gives 
$V_{12}, E_{12}, s_{12}, \ldots$, and to $\Phi_{12,1}, \Phi_{12,2}$ gives $\phi_{12,1}: V_{12} \rightarrow V_{1}=V, \chi_{12,1}, \xi_{12,1}$ and $\phi_{12,2}: V_{12} \rightarrow V_{2}, \chi_{12,2}, \xi_{12,2}$, simplifying notation a little. Set $v_{12}=\psi_{12}^{-1}(x) \in$ $s_{12}^{-1}(0) \subseteq V_{12}$, so that $\phi_{12,1}\left(v_{12}\right)=v_{1}$ and $\phi_{12,2}\left(v_{12}\right)=v_{2}$.

We have $\left(U, E^{-}\right)$satisfying $(*)$ for $A_{1}^{\bullet}, \boldsymbol{\alpha}_{1}, V_{1}, E_{1}, s_{1}, \ldots$ Thus by Lemma 3.12, we can choose $\left(U_{12}, E_{12}^{-}\right)$satisfying $(*)$ for $V_{12}, E_{12}, s_{12}, \ldots$ and compatible with $\left(U, E^{-}\right)$under $\phi_{12,1}, \chi_{12,1}$ in the sense of $\$ 3.4$, such that $v_{12} \in s_{12}^{-1}(0) \cap$ $\phi_{12,1}^{-1}(U) \subseteq U_{12} \subseteq V_{12}$. Also 33.4 defines $\chi_{12,1}^{+}$such that if $\left(U, E^{-}\right)$and $\left(U_{12}, E_{12}^{-}\right)$ satisfy $(\dagger)$ (we do not assume this), then

$$
\left(U_{12},\left.\phi_{12,1}\right|_{U_{12}}, \chi_{12,1}^{+}\right):\left(U_{12}, E_{12}^{+}, s_{12}^{+}, \psi_{12}^{+}\right) \longrightarrow\left(U, E^{+}, s^{+}, \psi^{+}\right)
$$

is a coordinate change of Kuranishi neighbourhoods, as in Corollary 3.11

Now apply Definition 5.1 to pushforward $\left(U_{12}, E_{12}^{-}\right)$in $V_{12}, E_{12}, s_{12}, \ldots$ along $\phi_{12,2}, \chi_{12,2}, \xi_{12,2}$. This yields $\left(U_{2}, E_{2}^{-}\right)$satisfying $(*)$ for $V_{2}, E_{2}, s_{2}, \ldots$ with $\phi_{12,2}\left(s_{12}^{-1}(0) \cap U_{12}\right) \subseteq U_{2} \subseteq V_{2}$, so in particular $v_{2} \in U_{2}$, and data $\theta_{2,12}, \eta_{2,12}$ such that if $\left(U_{2}, E_{2}^{-}\right)$and $\left(U_{12}, E_{12}^{-}\right)$satisfy ( $\left.\dagger\right)$ (we do not assume this), then

$$
\left(U_{2}, \theta_{2,12}, \eta_{2,12}\right):\left(U_{2}, E_{2}^{+}, s_{2}^{+}, \psi_{2}^{+}\right) \longrightarrow\left(U_{12}, E_{12}^{+}, s_{12}^{+}, \psi_{12}^{+}\right)
$$

is a coordinate change of Kuranishi neighbourhoods, as in (5.8).

Since $\left(A_{2}^{\bullet}, \omega_{A_{2}^{*}}\right)$ is in -2 -Darboux form and minimal at $x$, Example 3.8 proves that there exists an open neighbourhood $U_{2}^{\prime}$ of $v_{2}$ in $U_{2}$ such that $s_{2}^{-1}(0) \cap U_{2}^{\prime}=$ $\left(s_{2}^{+}\right)^{-1}(0) \cap U_{2}^{\prime}$. Then $\left(U_{2}^{\prime},\left.E_{2}^{-}\right|_{U_{2}^{\prime}}\right)$ satisfies $(\dagger)$. The construction in Definition 5.1 implies that $\theta_{2,12}$ identifies $s_{2}^{-1}(0)$ near $v_{2}$ with $s_{12}^{-1}(0)$ near $v_{12}$, and identifies $\left(s_{2}^{+}\right)^{-1}(0)$ near $v_{2}$ with $\left(s_{12}^{+}\right)^{-1}(0)$ near $v_{12}$ (the second follows from the fact that the analogue of (2.8) for $\theta_{2,12}, \eta_{2,12}$ at $v_{2}, v_{12}$ is exact, so (5.14) is a coordinate change of Kuranishi neighbourhoods near $\left.v_{2}, v_{12}\right)$. Since $s_{2}^{-1}(0)=\left(s_{2}^{+}\right)^{-1}(0)$ near $v_{2}$, it follows that $s_{12}^{-1}(0)=\left(s_{12}^{+}\right)^{-1}(0)$ near $v_{12}$. That is, there exists an open neighbourhood $U_{12}^{\prime}$ of $v_{12}$ in $U_{12}$ such that $s_{12}^{-1}(0) \cap U_{12}^{\prime}=\left(s_{12}^{+}\right)^{-1}(0) \cap U_{12}^{\prime}$.

Similarly, $\phi_{12,1}$ identifies $s_{12}^{-1}(0)$ near $v_{12}$ with $s^{-1}(0)$ near $v$, and identifies $\left(s_{12}^{+}\right)^{-1}(0)$ near $v_{12}$ with $\left(s^{+}\right)^{-1}(0)$ near $v$, so there exists an open neighbourhood $U_{v}^{\prime}$ of $v$ in $U$ such that $s^{-1}(0) \cap U_{v}^{\prime}=\left(s^{+}\right)^{-1}(0) \cap U_{v}^{\prime}$. This holds for all $v \in s^{-1}(0) \cap U$. Define $U^{\prime}=\bigcup_{v \in s^{-1}(0)} U_{v}^{\prime}$. Then $U^{\prime}$ is an open neighbourhood of $s^{-1}(0) \cap U$ in $U$, and $s^{-1}(0) \cap U^{\prime}=\left(s^{+}\right)^{-1}(0) \cap U^{\prime}$. Theorem 3.7 (c) follows.

\section{Proofs of some auxiliary results}

Next we prove Propositions $3.13,3.14$ and 3.17

\subsection{Proof of Proposition 3.13}

Let $Z$ be a paracompact, Hausdorff topological space and $\left\{R_{i}: i \in I\right\}$ an open cover of $Z$. By paracompactness we can choose a locally finite refinement $\left\{S_{i}: i \in I\right\}$. That is, $S_{i} \subseteq R_{i} \subseteq Z$ is open with $\bigcup_{i \in I} S_{i}=Z$, and each $z \in Z$ has an open $z \in U_{z} \subseteq Z$ with $U_{z} \cap S_{i} \neq \emptyset$ for only finitely many $i \in I$. 
By a standard result in topology known as the Shrinking Lemma, we can choose open sets $T_{i}^{1} \subseteq Z$ with closures $\bar{T}_{i}^{1} \subseteq Z$ for $i \in I$ such that $T_{i}^{1} \subseteq \bar{T}_{i}^{1} \subseteq S_{i}$ for $i \in I$ and $\bigcup_{i \in I} T_{i}^{1}=Z$. The next part of the proof broadly follows that of McDuff and Wehrheim [28, Lem. 7.1.7], who prove a similar result with $Z$ compact and $I$ finite. By induction on $k=2,3, \ldots$, choose open $T_{i}^{k} \subseteq Z$ with

$$
T_{i} \subseteq \bar{T}_{i}^{1} \subseteq T_{i}^{2} \subseteq \bar{T}_{i}^{2} \subseteq T_{i}^{3} \subseteq \bar{T}_{i}^{3} \subseteq \cdots \subseteq S_{i} \subseteq Z
$$

for $i \in I$. Here to choose $T_{i}^{k}$ we note that $Z$ is normal as it is paracompact and Hausdorff, so we can choose open $T_{i}^{k}, U \subseteq Z$ with $\bar{T}_{i}^{k-1} \subseteq T_{i}^{k}, Z \backslash S_{i} \subseteq U$ and $T_{i}^{k} \cap U=\emptyset$. Then $T_{i}^{k} \subseteq Z \backslash U \subseteq S_{i}$, and $Z \backslash U$ is closed, so $\bar{T}_{i}^{k} \subseteq S_{i}$.

Now for each finite $\emptyset \neq J \subseteq I$, define a closed subset $C_{J} \subseteq Z$ by

$$
C_{J}=\bigcap_{j \in J} \bar{T}_{j}^{|J|} \backslash \bigcap_{i \in I \backslash J} T_{i}^{|J|+1} .
$$

Then part (i) of the proposition follows from $\bar{T}_{j}^{|J|} \subseteq S_{j} \subseteq R_{j}$ for $j \in J$ by (6.1), and (ii) from $\left\{S_{i}: i \in I\right\}$ locally finite with $C_{J} \subseteq \bigcap_{i \in I} S_{i}$. For (iii), suppose $\emptyset \neq J, K \subseteq I$ are finite with $J \nsubseteq K$ and $K \nsubseteq J$. Without loss of generality, suppose $|J| \leqslant|K|$. Then there exists $j \in J \backslash K$, and (6.2) gives $C_{J} \subseteq \bar{T}_{j}^{|J|}$ and $C_{K} \subseteq Z \backslash T_{j}^{|K|+1}$, which forces $C_{J} \cap C_{K}=\emptyset$ as $\bar{T}_{j}^{|J|} \subseteq T_{j}^{|K|+1}$ by (6.1).

For part (iv), if $z \in Z$, define

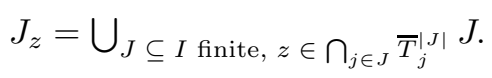

Then $J_{z}$ is finite as $\left\{S_{i}: i \in I\right\}$ is locally finite, so $z \in S_{j}$ for only finitely many $j \in I$, and $J_{z}$ is nonempty as $\left\{T_{i}^{1}: i \in I\right\}$ covers $Z$, so $z \in T_{i}^{1} \subseteq \bar{T}_{i}^{2}$ for some $i \in I$, and $J=\{i\}$ is a possible set in the union (6.3). If $j \in J_{z}$ then $j \in J$ for some $J$ in the union ([6.3), so that $z \in \bar{T}_{j}^{|J|} \subseteq \bar{T}_{j}^{\left|J_{z}\right|}$ as $|J| \leqslant\left|J_{z}\right|$. If $i \in I \backslash J_{z}$ then $z \notin \bigcap_{j \in J_{z} \cup\{i\}} \bar{T}_{j}^{\left|J_{z}\right|+1}$, as $J_{z} \cup\{i\}$ is not one of the sets $J$ in (6.3), but $z \in \bigcap_{j \in J_{z}} \bar{T}_{j}^{\left|J_{z}\right|+1}$, so $z \notin \bar{T}_{i}^{\left|J_{z}\right|+1}$. Hence $z \in C_{J_{z}}$ by (6.2), and part (iv) follows. This completes the proof of Proposition 3.13

\subsection{Proof of Proposition 3.14}

We work in the situation of 33.5 just after Remark 3.28, so that we have data $X_{\text {an }}, I, V_{J}, E_{J}, s_{J}, \psi_{J}$ and $C_{J} \subseteq R_{J}=\bigcap_{i \in J} R_{i} \subseteq X_{\text {an }}$ for all $J \in A$, and $\phi_{J K}, \chi_{J K}$ for all $J, K \in A$ with $K \subsetneq J$. We will first prove the following inductive hypothesis $(+)_{m}$, by induction on $m=1,2, \ldots$ :

$(+)_{m}$ For all $J \in A$ with $|J| \leqslant m$, we can choose $\left(\tilde{U}_{J}, \tilde{E}_{J}^{-}\right)$satisfying condition $(*)$ for $A_{J}^{\bullet}, V_{J}, E_{J}, F_{J}, s_{J}, t_{J}, \psi_{J}, \ldots$, such that $\psi_{J}^{-1}\left(C_{J}\right) \subseteq \tilde{U}_{J} \subseteq V_{J}$, and if $J, K \in A$ with $K \subsetneq J$ and $0<|K|<|J| \leqslant m$ then there exists open $\tilde{U}_{J K} \subseteq \tilde{U}_{J}$ with $\psi_{J}^{-1}\left(C_{J} \cap C_{K}\right) \subseteq \tilde{U}_{J K}$ such that $\left(\tilde{U}_{J K},\left.\tilde{E}_{J}^{-}\right|_{\tilde{U}_{J K}}\right)$ is compatible with $\left(\tilde{U}_{K}, \tilde{E}_{K}^{-}\right)$, in the sense of 3.4 That is, $\phi_{J K}\left(\tilde{U}_{J K}\right) \subseteq$ $\tilde{U}_{K} \subseteq V_{K}$ and $\left.\left.\left.\chi_{J K}\right|_{\tilde{U}_{J K}}\left(\left.\tilde{E}_{J}^{-}\right|_{\tilde{U}_{J K}}\right) \subseteq \phi_{J K}\right|_{\tilde{U}_{J K}} ^{*}\left(\tilde{E}_{K}^{-}\right) \subseteq \phi_{J K}\right|_{\tilde{U}_{J K}} ^{*}\left(E_{K}\right)$. 
For the first step, to prove $(+)_{1}$, for all $J=\{i\}$ with $i \in I$ we choose $\left(\tilde{U}_{J}, \tilde{E}_{J}^{-}\right)$ for $A_{J}^{\bullet}, V_{J}, E_{J}, \ldots$ satisfying (*) with $s_{J}^{-1}(0) \subseteq \tilde{U}_{J}$, so that $\psi_{J}^{-1}\left(C_{J}\right) \subseteq \tilde{U}_{J}$, by applying Theorem 3.7 (b) with $C=U=\emptyset$. The second part of $(+)_{1}$ is trivial, as there are no $J, K \in A$ with $0<|K|<|J| \leqslant 1$.

For the inductive step, suppose $(+)_{m-1}$ holds for some $m>1$. We will prove $(+)_{m}$. Using the existing choices of $\left(\tilde{U}_{J}, \tilde{E}_{J}^{-}\right)$and $\tilde{U}_{J K}$ for $J, K \in A$ with $|J|,|K|<m$ from $(+)_{m-1}$, it remains to choose $\left(\tilde{U}_{J}, \tilde{E}_{J}^{-}\right)$when $|J|=m$, and $\tilde{U}_{J K}$ when $0<|K|<|J|=m$. So fix $J \subseteq I$ with $|J|=m$.

Then $(+)_{m-1}$ gives $\left(\tilde{U}_{K}, \tilde{E}_{K}^{-}\right)$satisfying $(*)$ for all $\emptyset \neq K \subsetneq J$. Using the notation of Lemma 3.12, set $\tilde{U}_{J K}^{\prime}=\phi_{J K}^{-1}\left(\tilde{U}_{K}\right) \subseteq V_{J}$, and define $\tilde{E}_{J K}^{\prime}=$ $\left.\chi_{J K}\right|_{\tilde{U}_{J K}^{\prime}} ^{-1}\left(\tilde{E}_{K}^{-}\right)$, a vector subbundle of $\left.E_{J}\right|_{\tilde{U}_{J K}^{\prime}}$. Then $\tilde{U}_{J K}^{\prime}$ is an open neighbourhood of $\psi_{J}^{-1}\left(C_{K}\right)$ in $V_{J}$, by (3.16).

If $\emptyset \neq L \subsetneq K \subsetneq J$ then by $(+)_{m-1}$ there exists open $\tilde{U}_{K L} \subseteq \tilde{U}_{K}$ with $\psi_{K}^{-1}\left(C_{K} \cap C_{L}\right) \subseteq \tilde{\tilde{U}}_{K L}$ such that $\phi_{K L}\left(\tilde{U}_{K L}\right) \subseteq \tilde{U}_{L}$ and $\left.\chi_{K L}\right|_{\tilde{U}_{K L}}\left(\tilde{E}_{K}^{-}\right) \subseteq$ $\left.\left.\phi_{K L}\right|_{\tilde{U}_{K L}} ^{*}\left(\tilde{E}_{L}^{-}\right) \subseteq \phi_{K L}\right|_{\tilde{U}_{K L}} ^{*}\left(\tilde{E}_{L}\right)$. Pulling back by $\phi_{J K}$, applying $\chi_{J K}$, and using the last part of Corollary 3.5(ii) then shows that we have an open neighbourhood $\tilde{U}_{J K L}^{\prime}=\phi_{J K}^{-1}\left(\tilde{U}_{K L}\right)$ of $\psi_{J}^{-1}\left(C_{K} \cap C_{L}\right)$ in $\tilde{U}_{J K}^{\prime} \cap \tilde{U}_{J L}^{\prime} \subseteq V_{J}$, such that

$$
\left.\left.\left.\tilde{E}_{J K}^{\prime}\right|_{\tilde{U}_{J K L}^{\prime}} \subseteq \tilde{E}_{J L}^{\prime}\right|_{\tilde{U}_{J K L}^{\prime}} \subseteq E_{J}\right|_{\tilde{U}_{J K L}^{\prime}} .
$$

As in Lemma 3.12, choose vector subbundles $\left.\tilde{E}_{J K}^{\prime \prime} \subseteq E_{J}\right|_{\tilde{U}_{J K}^{\prime}}$ with $\left.E_{J}\right|_{\tilde{U}_{J K}^{\prime}}=$ $\tilde{E}_{J K}^{\prime} \oplus \tilde{E}_{J K}^{\prime \prime}$ on $\tilde{U}_{J K}^{\prime}$ for all $\emptyset \neq K \subsetneq J$. Choose a connection $\nabla$ on $E_{J}$. As in Lemma 3.12, for all $\emptyset \neq K \subsetneq J, \tilde{E}_{J K}^{\prime \prime \prime}:=\left(\nabla s_{J}\right)\left[\operatorname{Kerd} \phi_{J K}\right]$ is a vector subbundle of $E_{J}$ near $s_{J}^{-1}(0)$ in $V_{J}$. Making the open neighbourhoods $\tilde{U}_{J K}^{\prime}, \tilde{U}_{J K L}^{\prime}$ smaller, we can suppose $\tilde{E}_{J K}^{\prime \prime \prime}$ is a vector subbundle of $\left.E_{J}\right|_{\tilde{U}_{J K}^{\prime}}$. If $\emptyset \neq L \subsetneq K \subsetneq J \subseteq I$ then $\operatorname{Ker} \mathrm{d} \phi_{J K} \subseteq \operatorname{Kerd} \phi_{J L}$, as $\phi_{J L}=\phi_{K L} \circ \phi_{J K}$, and so

$$
\left.\left.\left.\tilde{E}_{J K}^{\prime \prime \prime}\right|_{\tilde{U}_{J K L}^{\prime}} \subseteq \tilde{E}_{J L}^{\prime \prime \prime}\right|_{\tilde{U}_{J K L}^{\prime}} \subseteq E_{J}\right|_{\tilde{U}_{J K L}^{\prime}} .
$$

Next, by reverse induction on $l=m-1, m-2, \ldots, 1$, we will prove the following inductive hypothesis $(\times)_{J, l}$ :

$(\times)_{J, l}$ For all $\emptyset \neq L \subsetneq J$ with $l \leqslant|L|$ we can choose an open neighbourhood $\hat{U}_{J L}$ of $\psi_{J}^{-1}\left(C_{J} \cap C_{L}\right)$ in $\tilde{U}_{J L}$ and a vector subbundle $\hat{E}_{J L}^{-}$of $E_{J L}^{\prime} \mid \hat{U}_{J L}$ such that

$$
\left.E_{J}\right|_{\hat{U}_{J L}}=\left.\left.\hat{E}_{J L}^{-} \oplus E_{J L}^{\prime \prime}\right|_{\hat{U}_{J L}} \oplus E_{J L}^{\prime \prime \prime}\right|_{\hat{U}_{J L}},
$$

or equivalently, identifying $E_{J L}^{\prime}$ with $E_{J} / E_{J L}^{\prime \prime}$ on $\hat{U}_{J L}$,

$$
\left.E_{J L}^{\prime}\right|_{\hat{U}_{J L}}=\left.\hat{E}_{J L}^{-} \oplus\left[\left(E_{J L}^{\prime \prime} \oplus E_{J L}^{\prime \prime \prime}\right) / E_{J L}^{\prime \prime}\right]\right|_{\hat{U}_{J L}},
$$

and such that if $\emptyset \neq L \subsetneq K \subsetneq J$ with $l \leqslant|L|<|K|$ then there exists an open neighbourhood $\hat{U}_{J K L}$ of $\psi_{J}^{-1}\left(C_{J} \cap C_{K} \cap C_{L}\right)$ in $\hat{U}_{J K} \cap \hat{U}_{J L}$ with $\left.\hat{E}_{J L}^{-}\right|_{\hat{U}_{J K L}}=\left.\hat{E}_{J K}^{-}\right|_{\hat{U}_{J K L}}$. 
For the first step $l=m-1$, for each $L \subsetneq J$ with $|L|=m-1$ we take $\hat{U}_{J L}=\tilde{U}_{J L}$ and take $\hat{E}_{J L}^{-}$to be an arbitrary complement to $\left[\left(E_{J L}^{\prime \prime} \oplus E_{J L}^{\prime \prime \prime}\right) / E_{J L}^{\prime \prime}\right]$ in $\left.E_{J L}^{\prime}\right|_{\tilde{U}_{J L}}$, as in (6.5), which implies (6.4). The second part of $(\times)_{J, m-1}$ is trivial as there are no $K, L$ with $m-1 \leqslant|L|<|K|<|J|=m$.

For the inductive step, suppose $(\times)_{J, l+1}$ holds for some $1 \leqslant l<m-1$, and fix $L \subsetneq J$ with $|L|=l$. Choose open neighbourhoods $\hat{U}_{J K L}$ of $\psi_{J}^{-1}\left(C_{J} \cap C_{K} \cap C_{L}\right)$ in $V_{J}$ for all $L \subsetneq K \subsetneq J$ with the properties that:

(a) $\hat{U}_{J K L} \subseteq \hat{U}_{J K} \cap \tilde{U}_{J L}$, where $\hat{U}_{J K}$ is already chosen by $(\times)_{J, l+1}$.

(b) If $L \subsetneq K_{1}, K_{2} \subsetneq J$ with $K_{1} \subsetneq K_{2}$ and $K_{2} \subsetneq K_{1}$ then $\hat{U}_{J K_{1} L} \cap \hat{U}_{J K_{2} L}=\emptyset$.

(c) If $L \subsetneq K_{2} \subsetneq K_{1} \subsetneq J$ then $\hat{U}_{J K_{1} L} \cap \hat{U}_{J K_{2} L} \subseteq \hat{U}_{J K_{1} K_{2}}$, where $\hat{U}_{J K_{1} K_{2}}$ is already chosen by $(\times)_{J, l+1}$.

This is possible, using Proposition 3.13(iii) to ensure (b).

Next, we have to choose an open neighbourhood $\hat{U}_{J L}$ of $\psi_{J}^{-1}\left(C_{J} \cap C_{L}\right)$ in $\tilde{U}_{J L}$ and a vector subbundle $\hat{E}_{J L}^{-}$of $\left.E_{J L}^{\prime}\right|_{U_{J L}}$ satisfying (6.4)-(6.5), such that for all $K$ with $L \subsetneq K \subsetneq J$ we have $\hat{U}_{J K L} \subseteq \hat{U}_{J L}$ and $\left.\hat{E}_{J L}^{-}\right|_{\hat{U}_{J K L}}=\left.\hat{E}_{J K}^{-}\right|_{\hat{U}_{J K L}}$.

First note from Lemma 3.12 that (6.4) -(6.5) near $\psi_{J}^{-1}\left(C_{J} \cap C_{L}\right)$ are equivalent to $\left(\hat{U}_{J L}, \hat{E}_{J L}^{-}\right)$near $\psi_{J}^{-1}\left(C_{J} \cap C_{L}\right)$ satisfying $(*)$ and being compatible with $\left(\tilde{U}_{L}, \tilde{E}_{L}^{-}\right)$. By $(\times)_{J, l+1}$ we already know that $\left.\hat{E}_{J K}^{-}\right|_{\hat{U}_{J K L}}$ near $\psi_{J}^{-1}\left(C_{J} \cap C_{L}\right)$ satisfies $(*)$ and is compatible with $\left(\tilde{U}_{K}, \tilde{E}_{K}^{-}\right)$, so $\left.\hat{E}_{J K}^{-}\right|_{\hat{U}_{J K L}}$ is compatible with $\left(\tilde{U}_{L}, \tilde{E}_{L}^{-}\right)$near $\psi_{J}^{-1}\left(C_{J} \cap C_{L}\right)$ as $\left(\tilde{U}_{K}, \tilde{E}_{K}^{-}\right)$is compatible with $\left(\tilde{U}_{L}, \tilde{E}_{L}^{-}\right)$by $(+)_{m-1}$. Therefore the prescribed value $\left.\hat{E}_{J K}^{-}\right|_{\hat{U}_{J K L}}$ for $\hat{E}_{J L}^{-}$on $\hat{U}_{J K L}$ satisfies (6.4) - 6.5) near $\psi_{J}^{-1}\left(C_{J} \cap C_{L}\right)$, and making $\hat{U}_{J K L}$ smaller, we can suppose $\left.\hat{E}_{J K}^{-}\right|_{\hat{U}_{J K L}}$ satisfies (6.4)-(6.5) on $\hat{U}_{J K L}$. This proves that (6.4)-6.5) are compatible with the conditions $\left.\hat{E}_{J L}^{-}\right|_{\hat{U}_{J K L}}=\left.\hat{E}_{J K}^{-}\right|_{\hat{U}_{J K L}}$ for all $\emptyset \neq L \subsetneq K \subsetneq J$.

Next, observe that the prescribed values $\left.\hat{E}_{J K}^{-}\right|_{\hat{U}_{J K L}}$ for $\hat{E}_{J L}^{-}$on $\hat{U}_{J K L}$ for different $K_{1}, K_{2}$ with $L \subsetneq K_{1}, K_{2} \subsetneq J$ agree on overlaps $\hat{U}_{J K_{1} L} \cap \hat{U}_{J K_{2} L}$. This follows from (b),(c) above and $\left.\hat{E}_{J K_{1}}^{-}\right|_{\hat{U}_{J K_{1} K_{2}}}=\left.\hat{E}_{J K_{2}}^{-}\right|_{\hat{U}_{J K_{1} K_{2}}}$, which holds by $(\times)_{J, l+1}$. Therefore the last part of $(\times)_{J, l}$ can be rewritten to say that we have one prescribed value for $\hat{E}_{J L}^{-}$on the subset $\dot{U}_{J L}:=\bigcup_{K: L \subsetneq K \subsetneq J} \hat{U}_{J K L}$, which satisfies (6.4)-(6.5) on $\dot{U}_{J L}$.

So, we are given a prescribed value of $\hat{E}_{J L}^{-}$on an open set $\dot{U}_{J L} \subseteq V_{J}$ satisfying (6.5), and we have to extend it to a larger open set $\hat{U}_{J L} \subseteq V_{J}$ containing both $\dot{U}_{J L}$ and $\psi_{J}^{-1}\left(C_{J} \cap C_{K} \cap C_{L}\right)$. This may not be possible: if we have chosen previous $\hat{E}_{J K}^{-}$'s badly near the 'edge' of $\dot{U}_{J L}$ in $V_{J}$, then the prescribed values of $\hat{E}_{J L}^{-}$may not extend continuously to the closure $\overline{\dot{U}_{J L}}$ of $\dot{U}_{J L}$ in $V_{J}$, and in particular, may not extend continuously over points in $\left[\psi_{J}^{-1}\left(C_{J} \cap C_{K} \cap C_{L}\right)\right] \cap$ $\left[\overline{\dot{U}_{J L}} \backslash \dot{U}_{J L}\right]$. However, we can deal with this problem by shrinking all the $\hat{U}_{J K L}$ 's, such that the closure $\overline{\dot{U}_{J L}}$ of the new $\dot{U}_{J L}$ lies inside the old $\dot{U}_{J L}$. Then it is guaranteed that the prescribed value of $\hat{E}_{J L}^{-}$on $\dot{U}_{J L}$ extends smoothly to an open neighbourhood of $\overline{\dot{U}_{J L}}$ in $V_{J}$, so we can choose $\left(\hat{U}_{J L}, \hat{E}_{J L}^{-}\right)$satisfying all 
the required conditions. As this holds for all $L \subsetneq J$ with $|L|=l$, this completes the inductive step, and $(\times)_{J, l}$ holds for all $l=m-1, m-2, \ldots, 1$.

Fix data $\hat{U}_{J L}, \hat{E}_{J L}^{-}, \hat{U}_{J K L}$ as in $(\times)_{J, 1}$. For all $\emptyset \neq K \subsetneq J$, choose open neighbourhoods $\check{U}_{J K}$ of $\psi_{J}^{-1}\left(C_{J} \cap C_{K}\right)$ in $\hat{U}_{J K}$ such that if $K_{1} \subsetneq K_{2}$ and $K_{2} \subsetneq K_{1}$ then $\check{U}_{J K_{1}} \cap \check{U}_{J K_{2}}=\emptyset$, and if $\emptyset \neq L \subsetneq K \subsetneq J$ then $\check{U}_{J K} \cap \check{U}_{J L} \subseteq \hat{U}_{J K L}$. This is possible provided the $\check{U}_{J K}$ are small enough, using Proposition 3.13 (iii) to ensure $\check{U}_{J K_{1}} \cap \check{U}_{J K_{2}}=\emptyset$.

Define $\breve{U}_{J}=\bigcup_{K: \emptyset \neq K \subsetneq J} \check{U}_{J K}$. It is an open neighbourhood of the closed

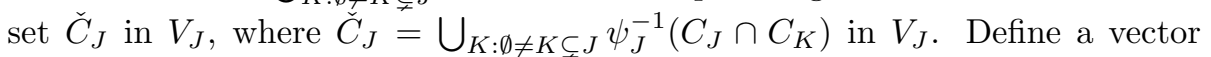
subbundle $\check{E}_{J}^{-}$of $\left.E_{J}\right|_{\check{U}_{J}}$ by $\left.\check{E}_{J}^{-}\right|_{\check{U}_{J K}}=\left.\hat{E}_{J L}^{-}\right|_{\check{U}_{J K}}$ for all $\emptyset \neq K \subsetneq J$. These prescribed values for different $K_{1}, K_{2}$ are compatible on the overlap $\check{U}_{J K_{1}} \cap \check{U}_{J K_{2}}$ by construction, so $\check{E}_{J}^{-}$is well-defined.

Now apply Theorem 3.7(b) to $A_{J}^{\bullet}, V_{J}, E_{J}, s_{J}, \ldots$, with closed set $\check{C}_{J} \subseteq V_{J}$ and pair $\left(\check{U}_{J}, \check{E}_{J}^{-}\right)$satisfying $(*)$ with $\check{C}_{J} \subseteq \check{U}_{J}$. This shows that there exists $\left(\tilde{U}_{J}, \tilde{E}_{J}^{-}\right)$satisfying $(*)$ for $A_{J}^{\bullet}, V_{J}, E_{J}, s_{J}, \ldots$, and an open neighbourhood $\check{U}_{J}^{\prime}$ of $\check{C}_{J}$ in $\check{U}_{J} \cap \tilde{U}_{J}$ such that $\left.\check{E}_{J}^{-}\right|_{\check{U}_{J}^{\prime}}=\left.\tilde{E}_{J}^{-}\right|_{\check{U}_{J}^{\prime}}$. For all $\emptyset \neq K \subsetneq J$, set $\tilde{U}_{J K}=$ $\check{U}_{J}^{\prime} \cap \check{U}_{J K}$. Then $\tilde{U}_{J K}$ is an open neighbourhood of $\psi_{J}^{-1}\left(C_{J} \cap C_{K}\right)$ in $V_{J}$, and $\left.\tilde{E}_{J}^{-}\right|_{\tilde{U}_{J K}}=\left.\check{E}_{J}^{-}\right|_{\tilde{U}_{J K}}=\left.\hat{E}_{J K}^{-}\right|_{\tilde{U}_{J K}}$, which is compatible with $\left(\tilde{U}_{K}, \tilde{E}_{K}^{-}\right)$by definition. This completes the proof of the inductive step of $(+)_{m}$. So by induction, $(+)_{m}$ holds for all $m=1,2, \ldots$.

Fix data $\left(\tilde{U}_{J}, \tilde{E}_{J}^{-}\right)$for all $J \in A$ and $\tilde{U}_{J K}$ for all $J, K \in A$ with $K \subsetneq J$ as in $(+)_{m}$ as $m \rightarrow \infty$ (or $m=|I|$ if $I$ is finite). For all $J \in A$, choose open neighbourhoods $U_{J}$ of $\psi_{J}^{-1}\left(C_{J}\right)$ in $\tilde{U}_{J}$, such that setting $E_{J}^{-}=\left.\tilde{E}_{J}^{-}\right|_{U_{J}}$ and $S_{J}=\psi_{J}\left(s_{J}^{-1}(0) \cap U_{J}\right)$, so that $S_{J}$ is an open neighbourhood of $C_{J}$ in $X_{\text {an }}$, then $\left(U_{J}, E_{J}^{-}\right)$satisfies condition ( $\dagger$ ), and for all $J, K \in A$, if $J \nsubseteq K$ and $K \nsubseteq J$ then $S_{J} \cap S_{K}=\emptyset$, and if $K \subsetneq J$ then $\psi_{J}^{-1}\left(S_{J} \cap S_{K}\right) \subseteq \tilde{U}_{J K}$. If $K \subsetneq J$, we define $U_{J K}=\tilde{U}_{J K} \cap U_{J} \cap \phi_{J K}^{-1}\left(U_{K}\right)$. Then $s_{J}^{-1}(0) \cap U_{J K}=\psi_{J}^{-1}\left(S_{J} \cap S_{K}\right)$, and $\left(U_{J K},\left.E_{J}^{-}\right|_{U_{J K}}\right)$ is compatible with $\left(U_{K}, E_{K}^{-}\right)$.

To see that we can choose $U_{J}$ for all $J \in A$ satisfying all these conditions, note that by Theorem 3.7 (c), if $U_{J}$ is small enough then $\left(U_{J}, E_{J}^{-}\right)$satisfies $(\dagger)$, as $\left(\tilde{U}_{J}, \tilde{E}_{J}^{-}\right)$satisfies $(*)$. If $J \nsubseteq K$ and $K \nsubseteq \subseteq J$ then Proposition 3.13(iii) implies that $S_{J} \cap S_{K}=\emptyset$ provided both $U_{J}, U_{K}$ are sufficiently small. Similarly, if $K \subsetneq J$ then $\psi_{J}^{-1}\left(S_{J} \cap S_{K}\right) \subseteq \tilde{U}_{J K}$ holds provided both $U_{J}, U_{K}$ are sufficiently small. Now if $I$ is infinite, it is possible that an individual set $U_{J}$ may have to satisfy infinitely many smallness conditions, for compatibility with infinitely many sets $\emptyset \neq K \subseteq I$. However, the local finiteness condition Proposition 3.13(ii) means that in an open neighbourhood of any $v_{J} \in \psi_{J}^{-1}\left(C_{J}\right)$, only finitely many smallness conditions on $U_{J}$ are relevant, so we can solve them. This completes the proof of Proposition 3.14 .

\subsection{Proof of Proposition 3.17}

Let $\left(\boldsymbol{X}, \omega_{\boldsymbol{X}}^{*}\right), X_{\mathrm{an}}, \mathcal{K}$ and $\boldsymbol{X}_{\mathrm{dm}}$ be as in Theorems 3.15 and 3.16, and use the notation of $₫ 3.5$. First we relate orientations on $\left(\boldsymbol{X}, \omega_{\boldsymbol{X}}^{*}\right)$ and $\boldsymbol{X}_{\mathrm{dm}}$ at one point 
$x \in X_{\text {an }}$. Pick $J \in A$ with $x \in S_{J}=\operatorname{Im} \psi_{J}^{+}$. From (2.7) and (2.9) we have

$\left\{\right.$ orientations on $\left(\boldsymbol{X}, \omega_{\boldsymbol{X}}^{*}\right)$ at $\left.x\right\} \cong\left\{\mathbb{C}\right.$-orientations on $\left.\left(H^{1}\left(\left.\mathbb{T}_{\boldsymbol{X}}\right|_{x}\right), Q_{x}\right)\right\}$,

$\left\{\right.$ orientations on $\boldsymbol{X}_{\mathrm{dm}}$ at $\left.x\right\} \cong\left\{\right.$ orientations on $\left.T_{x}^{*} \boldsymbol{X}_{\mathrm{dm}} \oplus O_{x} \boldsymbol{X}_{\mathrm{dm}}\right\}$,

where $Q_{x}=\omega_{\boldsymbol{X}}^{0}$. is the nondegenerate complex quadratic form on $H^{1}\left(\left.\mathbb{T}_{\boldsymbol{X}}\right|_{x}\right)$ in (2.6). There is a unique $v_{J}$ in $s_{J}^{-1}(0) \cap U_{J}=\left(s_{J}^{+}\right)^{-1}(0) \subseteq U_{J} \subseteq V_{J}$ with $\psi_{J}\left(v_{J}\right)=x$. Equation (3.9) gives an isomorphism of complex vector spaces

$$
H^{1}\left(\mathbb{T}_{\left.\boldsymbol{\alpha}_{J}\right|_{v_{J}}}\right): \frac{\operatorname{Ker}\left(\left.t_{J}\right|_{v_{J}}:\left.\left.E_{J}\right|_{v_{J}} \rightarrow F_{J}\right|_{v_{J}}\right)}{\operatorname{Im}\left(\left.\mathrm{d} s_{J}\right|_{v_{J}}:\left.T_{v_{J}} V_{J} \rightarrow E_{J}\right|_{v_{J}}\right)} \rightarrow H^{1}\left(\left.\mathbb{T}_{\boldsymbol{X}}\right|_{x}\right)
$$

Write $\tilde{Q}_{v_{J}}$ for the complex quadratic form on $\operatorname{Ker}\left(\left.t_{J}\right|_{v_{J}}\right) / \operatorname{Im}\left(\left.\mathrm{d} s_{J}\right|_{v_{J}}\right)$ identified with $Q_{x}$ by (6.8), as in Definition 3.6. Then by (6.6) we have

$$
\begin{aligned}
& \left\{\text { orientations on }\left(\boldsymbol{X}, \omega_{\boldsymbol{X}}^{*}\right) \text { at } x\right\} \cong \\
& \left\{\mathbb{C} \text {-orientations on }\left(\operatorname{Ker}\left(\left.t_{J}\right|_{v_{J}}\right) / \operatorname{Im}\left(\left.\mathrm{d} s_{J}\right|_{v_{J}}\right), \tilde{Q}_{v_{J}}\right)\right\} .
\end{aligned}
$$

Condition $(*)$ for $\left(U_{J}, E_{J}^{-}\right)$at $v_{J}$ requires that

$$
\Pi_{v_{J}}:\left.E_{J}^{-}\right|_{v_{J}} \cap \operatorname{Ker}\left(\left.t_{J}\right|_{v_{J}}:\left.\left.E_{J}\right|_{v_{J}} \rightarrow F_{J}\right|_{v_{J}}\right) \longrightarrow \frac{\operatorname{Ker}\left(\left.t_{J}\right|_{v_{J}}:\left.\left.E_{J}\right|_{v_{J}} \rightarrow F_{J}\right|_{v_{J}}\right)}{\operatorname{Im}\left(\left.\mathrm{d} s_{J}\right|_{v_{J}}:\left.T_{v_{J}} V_{J} \rightarrow E_{J}\right|_{v_{J}}\right)}
$$

should be injective, with image $\operatorname{Im} \Pi_{v_{J}}$ a real vector subspace of half the real dimension of $\operatorname{Ker}\left(\left.t_{J}\right|_{v_{J}}\right) / \operatorname{Im}\left(\left.\mathrm{d} s_{J}\right|_{v_{J}}\right)$, on which the real quadratic form $\operatorname{Re} \tilde{Q}_{v_{J}}$ is negative definite. As $\left(U_{J}, E_{J}^{+}, s_{J}^{+},\left.\psi_{J}\right|_{s_{J}^{-1}(0) \cap U_{J}}\right)$ is a Kuranishi neighbourhood on $\boldsymbol{X}_{\mathrm{dm}}$ by the proof of Theorem 3.16, equation (2.10) gives an exact sequence

$$
\left.0 \longrightarrow T_{x} \boldsymbol{X}_{\mathrm{dm}} \longrightarrow T_{v_{J}} V_{J} \stackrel{\left.\mathrm{d} s_{J}^{+}\right|_{v_{J}}}{\longrightarrow} E_{J}^{+}\right|_{v_{J}} \longrightarrow O_{x} \boldsymbol{X}_{\mathrm{dm}} \longrightarrow 0 .
$$

Condition $(*)$ implies that $\operatorname{Ker}\left(\left.\mathrm{d} s_{J}\right|_{v_{J}}\right)=\operatorname{Ker}\left(\left.\mathrm{d} s_{J}^{+}\right|_{v_{J}}\right)$, so we have

$$
T_{x} \boldsymbol{X}_{\mathrm{dm}} \cong \operatorname{Ker}\left(\left.\mathrm{d} s_{J}\right|_{v_{J}}:\left.T_{v_{J}} V_{J} \rightarrow E_{J}\right|_{v_{J}}\right) .
$$

Also from $(*)$ we see there is a canonical isomorphism

$$
O_{x} \boldsymbol{X}_{\mathrm{dm}} \cong \frac{\operatorname{Ker}\left(\left.t_{J}\right|_{v_{J}}\right) / \operatorname{Im}\left(\left.\mathrm{d} s_{J}\right|_{v_{J}}\right)}{\operatorname{Im} \Pi_{v_{J}}} .
$$

By (6.10), $T_{x} \boldsymbol{X}_{\mathrm{dm}}$ is a complex vector space, so $T_{x} \boldsymbol{X}_{\mathrm{dm}}$ and $T_{x}^{*} \boldsymbol{X}_{\mathrm{dm}}$ have natural orientations as real vector spaces. Thus by (6.11) we have a bijection

$$
\begin{aligned}
& \left\{\text { orientations on } T_{x}^{*} \boldsymbol{X}_{\mathrm{dm}} \oplus O_{x} \boldsymbol{X}_{\mathrm{dm}}\right\} \cong \\
& \left\{\text { orientations on }\left[\operatorname{Ker}\left(\left.t_{J}\right|_{v_{J}}\right) / \operatorname{Im}\left(\left.\mathrm{d} s_{J}\right|_{v_{J}}\right)\right] / \operatorname{Im} \Pi_{v_{J}}\right\} .
\end{aligned}
$$

Suppose we are given a complex basis $e_{1}, \ldots, e_{k}$ of $\operatorname{Ker}\left(\left.t_{J}\right|_{v_{J}}\right) / \operatorname{Im}\left(\left.\mathrm{d} s_{J}\right|_{v_{J}}\right) \cong$ $\mathbb{C}^{k}$ which is orthonormal w.r.t. $\tilde{Q}_{v_{J}}$. As $e_{1}, \ldots, e_{k}$ are orthonormal w.r.t. $\tilde{Q}_{v_{J}}$, the real quadratic form $\operatorname{Re} \tilde{Q}_{v_{J}}$ is positive definite on the real span $\left\langle e_{1}, \ldots, e_{k}\right\rangle_{\mathbb{R}}$, 
and $\operatorname{Re} \tilde{Q}_{v_{J}}$ is negative definite on $\operatorname{Im} \Pi_{v_{J}}$, so $\left\langle e_{1}, \ldots, e_{k}\right\rangle_{\mathbb{R}} \cap \operatorname{Im} \Pi_{v_{J}}=\{0\}$. Therefore $e_{1}+\operatorname{Im} \Pi_{v_{J}}, \ldots, e_{k}+\operatorname{Im} \Pi_{v_{J}}$ are linearly independent in the real vector space $\left[\operatorname{Ker}\left(\left.t_{J}\right|_{v_{J}}\right) / \operatorname{Im}\left(\left.\mathrm{d} s_{J}\right|_{v_{J}}\right)\right] / \operatorname{Im} \Pi_{v_{J}} \cong \mathbb{R}^{k}$, so they are a basis as $\operatorname{Im} \Pi_{v_{J}}$ has half the real dimension of $\operatorname{Ker}\left(\left.t_{J}\right|_{v_{J}}\right) / \operatorname{Im}\left(\left.\mathrm{d} s_{J}\right|_{v_{J}}\right)$. Define an identification

$$
\begin{aligned}
& \left\{\mathbb{C} \text {-orientations on }\left(\operatorname{Ker}\left(\left.t_{J}\right|_{v_{J}}\right) / \operatorname{Im}\left(\left.\mathrm{d} s_{J}\right|_{v_{J}}\right), \tilde{Q}_{v_{J}}\right)\right\} \\
& \quad \cong\left\{\text { orientations on }\left[\operatorname{Ker}\left(\left.t_{J}\right|_{v_{J}}\right) / \operatorname{Im}\left(\left.\mathrm{d} s_{J}\right|_{v_{J}}\right)\right] / \operatorname{Im} \Pi_{v_{J}}\right\}
\end{aligned}
$$

such that orientations on both sides are identified if, whenever $e_{1}, \ldots, e_{k}$ is an oriented orthonormal complex basis for $\left(\operatorname{Ker}\left(\left.t_{J}\right|_{v_{J}}\right) / \operatorname{Im}\left(\left.\mathrm{d} s_{J}\right|_{v_{J}}\right), \tilde{Q}_{v_{J}}\right)$, then $e_{1}+$ $\operatorname{Im} \Pi_{v_{J}}, \ldots, e_{k}+\operatorname{Im} \Pi_{v_{J}}$ is an oriented basis for $\left[\operatorname{Ker}\left(\left.t_{J}\right|_{v_{J}}\right) / \operatorname{Im}\left(\left.\mathrm{d} s_{J}\right|_{v_{J}}\right)\right] / \operatorname{Im} \Pi_{v_{J}}$. Combining equations (6.7), (6.9), (6.12) and (6.13) gives an identification

$$
\left\{\text { orientations on }\left(\boldsymbol{X}, \omega_{\boldsymbol{X}}^{*}\right) \text { at } x\right\} \cong\left\{\text { orientations on } \boldsymbol{X}_{\mathrm{dm}} \text { at } x\right\} \text {. }
$$

It is not difficult to show that the isomorphism (6.14) is independent of the choice of $J \in A$ with $x \in S_{J}$, and depends continuously on $x \in X_{\mathrm{an}}$. Thus we get a canonical 1-1 correspondence between the sets in Proposition 3.13(a),(c). The last part of Theorem 2.18 gives a 1-1 correspondence between the sets in Proposition 3.13(b),(c). This completes the proof.

\subsection{Proof of Proposition 3.18}

Suppose $\left(\boldsymbol{X}, \omega_{\boldsymbol{X}}^{*}\right)$ is a separated, -2 -shifted symplectic derived $\mathbb{C}$-scheme with $\operatorname{vdim}_{\mathbb{C}} \boldsymbol{X}=n$, whose complex analytic topological space $X_{\text {an }}$ is second countable. Let $\mathcal{K}, \mathcal{K}^{\prime}$ be different possible Kuranishi atlases constructed in Theorem 3.15, and $\boldsymbol{X}_{\mathrm{dm}}, \boldsymbol{X}_{\mathrm{dm}}^{\prime}$ the corresponding derived manifolds in Theorem 3.16,

As in 3.5 , let $\mathcal{K}$ be constructed using the family $\left\{\left(A_{i}^{\bullet}, \boldsymbol{\alpha}_{i}\right): i \in I\right\}$, and data $A_{J}^{\bullet}, \boldsymbol{\alpha}_{J}$ for $J \in A, \Phi_{J K}$ for $K \subseteq J$ in $A$ from Theorem 3.1, where $A=$ $\{J: \emptyset \neq J \subseteq I, J$ finite $\}$, as in 33.2 use notation $V_{J}, E_{J}, F_{J}, s_{J}, t_{J}, \psi_{J}$ and $R_{J}=\bigcap_{i \in J} R_{i} \subseteq X_{\text {an }}$ from $A_{J}^{\bullet}, \boldsymbol{\alpha}_{J}$ and $\phi_{J K}, \chi_{J K}, \xi_{J K}$ from $\Phi_{J K}$. Let $\mathcal{K}$ be defined using closed subsets $C_{J} \subseteq X_{\text {an }}$ for $J \in A$ in Proposition 3.13 and pairs $\left(U_{J}, E_{J}^{-}\right)$and open subsets $U_{J K} \subseteq U_{J}$ in Proposition 3.14 Similarly, let $\mathcal{K}^{\prime}$ be constructed using $\left\{\left(A_{i^{\prime}}^{\prime \bullet}, \boldsymbol{\alpha}_{i^{\prime}}^{\prime}\right): i^{\prime} \in I^{\prime}\right\}, A_{J^{\prime}}^{\prime \bullet}, \boldsymbol{\alpha}_{J^{\prime}}^{\prime}, V_{J^{\prime}}^{\prime}, E_{J^{\prime}}^{\prime}, \ldots, U_{J^{\prime} K^{\prime}}^{\prime} \subseteq U_{J^{\prime}}^{\prime}$.

We must build a derived manifold with boundary $\boldsymbol{W}_{\mathrm{dm}}$ with topological space $X_{\mathrm{an}} \times[0,1]$ and vdim $\boldsymbol{W}_{\mathrm{dm}}=n+1$, and an equivalence $\partial \boldsymbol{W}_{\mathrm{dm}} \simeq \boldsymbol{X}_{\mathrm{dm}} \amalg$ $\boldsymbol{X}_{\mathrm{dm}}^{\prime}$ topologically identifying $\boldsymbol{X}_{\mathrm{dm}}$ with $X_{\mathrm{an}} \times\{0\}$ and $\boldsymbol{X}_{\mathrm{dm}}^{\prime}$ with $X_{\mathrm{an}} \times\{1\}$.

Write $\tilde{\boldsymbol{\pi}}: \tilde{\boldsymbol{X}} \rightarrow Z$ to be the projection $\boldsymbol{\pi}_{\mathbb{A}^{1}}: \boldsymbol{X} \times \mathbb{A}^{1} \rightarrow \mathbb{A}^{1}$, so that $Z=\mathbb{A}^{1}=\operatorname{Spec} B$ with $B=\mathbb{C}[z]$, and $Z_{\text {an }}=\mathbb{C}$. Define $\omega_{\tilde{\boldsymbol{X}} / Z}=\boldsymbol{\pi}_{\boldsymbol{X}}^{*}\left(\omega_{\boldsymbol{X}}^{0}\right)$. Then $\omega_{\tilde{\boldsymbol{X}} / Z}$ is a family of -2 -shifted symplectic structures on $\boldsymbol{X} / Z$ in the sense of 33.7, the constant family over $Z=\mathbb{A}^{1}$ with fibre $\left(\boldsymbol{X}, \omega_{\boldsymbol{X}}^{*}\right)$. We now carry out the programme of 33.7 for $\tilde{\boldsymbol{\pi}}: \tilde{\boldsymbol{X}} \rightarrow Z, \omega_{\tilde{\boldsymbol{X}} / Z}$, choosing data as follows:

(a) Set $\tilde{I}=I \amalg I^{\prime}$, the disjoint union of $I$ and $I^{\prime}$.

(b) Define $\left(\tilde{A}_{i}^{\bullet}, \tilde{\boldsymbol{\alpha}}_{i}, \tilde{\beta}_{i}\right)$ for $i \in I$ by $\tilde{A}_{i}^{\bullet}=A_{i}^{\bullet} \otimes_{\mathbb{C}} \mathbb{C}\left[z,(z-1)^{-1}\right]$, so that $\operatorname{Spec} \tilde{A}_{i}^{\bullet}=\left(\operatorname{Spec} A_{i}^{\bullet}\right) \times\left(\mathbb{A}^{1} \backslash\{1\}\right)$, and $\tilde{\boldsymbol{\alpha}}_{i}=\boldsymbol{\alpha}_{i} \times \operatorname{inc}:\left(\operatorname{Spec} A_{i}^{\bullet}\right) \times$ 
$\left(\mathbb{A}^{1} \backslash\{1\}\right) \rightarrow \boldsymbol{X} \times \mathbb{A}^{1}$, and $\tilde{\beta}_{i}: \mathbb{C}[z] \rightarrow A_{i}^{0} \otimes_{\mathbb{C}} \mathbb{C}\left[z,(z-1)^{-1}\right], \tilde{\beta}_{i}: z \mapsto 1 \otimes z$. Similarly, define $\left(\tilde{A}_{i^{\prime}}^{\prime \bullet}, \tilde{\boldsymbol{\alpha}}_{i^{\prime}}, \tilde{\beta}_{i^{\prime}}\right)$ for $i^{\prime} \in I^{\prime}$ by $\tilde{A}_{i^{\prime}}^{\bullet}=A_{i^{\prime}}^{\prime \bullet} \otimes_{\mathbb{C}} \mathbb{C}\left[z, z^{-1}\right]$, so $\operatorname{Spec} \tilde{A}_{i^{\prime}}^{\prime \bullet}=\left(\operatorname{Spec} A_{i^{\prime}}^{\prime \bullet}\right) \times\left(\mathbb{A}^{1} \backslash\{0\}\right)$, and $\tilde{\boldsymbol{\alpha}}_{i^{\prime}}=\boldsymbol{\alpha}_{i^{\prime}}^{\prime} \times \operatorname{inc}:\left(\operatorname{Spec} A_{i^{\prime}}^{\prime \bullet}\right) \times$ $\left(\mathbb{A}^{1} \backslash\{0\}\right) \rightarrow \boldsymbol{X} \times \mathbb{A}^{1}$, and $\tilde{\beta}_{i^{\prime}}^{\prime}: \mathbb{C}[z] \rightarrow A_{i^{\prime}}^{\prime 0} \otimes_{\mathbb{C}} \mathbb{C}\left[z, z^{-1}\right], \tilde{\beta}_{i^{\prime}}^{\prime}: z \mapsto 1 \otimes z$.

(c) Write $\tilde{A}=\{\tilde{J}: \emptyset \neq \tilde{J} \subseteq \tilde{I}, \tilde{J}$ finite $\}$. Then $A \subseteq \tilde{A}$ and $A^{\prime} \subseteq \tilde{A}$.

(d) When we apply Theorem 3.1 to choose $\tilde{A}_{\tilde{J}}^{\bullet}, \tilde{\boldsymbol{\alpha}}_{\tilde{J}}, \tilde{\beta}_{\tilde{J}}$ for $\tilde{J} \in \tilde{A}$ and $\tilde{\Phi}_{\tilde{J} \tilde{K}}$ for $\tilde{K} \subseteq \tilde{J}$, we make these choices so that

$$
\begin{gathered}
\tilde{A}_{J}^{\bullet}=A_{J}^{\bullet} \otimes_{\mathbb{C}} \mathbb{C}\left[z,(z-1)^{-1}\right], \quad \tilde{A}_{J^{\prime}}^{\bullet}=A_{J^{\prime}}^{\prime} \otimes_{\mathbb{C}} \mathbb{C}\left[z, z^{-1}\right], \\
\tilde{\boldsymbol{\alpha}}_{J}=\boldsymbol{\alpha}_{J} \times \operatorname{inc}:\left(\operatorname{Spec} A_{J}^{\bullet}\right) \times\left(\mathbb{A}^{1} \backslash\{1\}\right) \longrightarrow \boldsymbol{X} \times \mathbb{A}^{1}, \tilde{\beta}_{J}: z \mapsto 1 \otimes z, \\
\tilde{\boldsymbol{\alpha}}_{J^{\prime}}=\boldsymbol{\alpha}_{J^{\prime}}^{\prime} \times \operatorname{inc}:\left(\operatorname{Spec} A_{J^{\prime}}^{\prime \bullet}\right) \times\left(\mathbb{A}^{1} \backslash\{0\}\right) \longrightarrow \boldsymbol{X} \times \mathbb{A}^{1}, \tilde{\beta}_{J^{\prime}}: z \mapsto 1 \otimes z, \\
\tilde{\Phi}_{J K}=\Phi_{J K} \otimes \operatorname{id}: A_{K}^{\bullet} \otimes_{\mathbb{C}} \mathbb{C}\left[z,(z-1)^{-1}\right] \longrightarrow A_{J}^{\bullet} \otimes_{\mathbb{C}} \mathbb{C}\left[z,(z-1)^{-1}\right], \\
\tilde{\Phi}_{J^{\prime} K^{\prime}}=\Phi_{J^{\prime} K^{\prime}}^{\prime} \otimes \operatorname{id}: A_{K^{\prime}}^{\prime \bullet} \otimes_{\mathbb{C}} \mathbb{C}\left[z, z^{-1}\right] \longrightarrow A_{J^{\prime}}^{\prime \bullet} \otimes_{\mathbb{C}} \mathbb{C}\left[z, z^{-1}\right],
\end{gathered}
$$

for all $K \subseteq J$ in $A$ and $K^{\prime} \subseteq J^{\prime}$ in $A^{\prime}$. This is clearly possible. Note that this does not determine $\tilde{A}_{\tilde{J}}^{\bullet}, \tilde{\boldsymbol{\alpha}}_{\tilde{J}}, \tilde{\beta}_{\tilde{J}}$ or $\Phi_{\tilde{J} \tilde{K}}$ if $\tilde{J} \in \tilde{A} \backslash\left(A \amalg A^{\prime}\right)$.

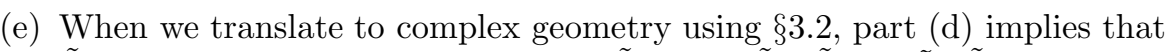
$\tilde{V}_{J}=V_{J} \times(\mathbb{C} \backslash\{1\})$ for $J \in A \subseteq \tilde{A}$. Also $\tilde{E}_{J}, \tilde{F}_{J}, \tilde{s}_{J}, \tilde{t}_{J}, \tilde{\phi}_{J K}, \tilde{\chi}_{J K}$ for $J, K \in A$ is obtained from $E_{J}, \ldots, \chi_{J K}$ by taking products with $\mathbb{C} \backslash\{1\}$. Similarly, $\tilde{V}_{J^{\prime}}, \tilde{E}_{J^{\prime}}, \tilde{F}_{J^{\prime}}, \tilde{s}_{J^{\prime}}, \tilde{t}_{J^{\prime}}, \tilde{\phi}_{J^{\prime} K^{\prime}}, \tilde{\chi}_{J^{\prime} K^{\prime}}$ for $J^{\prime}, K^{\prime} \in A^{\prime} \subseteq \tilde{A}$ are obtained from $V_{J^{\prime}}, \ldots, \chi_{J^{\prime} K^{\prime}}$ by taking products with $\mathbb{C} \backslash\{0\}$.

(f) When we choose data $\tilde{C}_{\tilde{J}},\left(\tilde{U}_{\tilde{J}}, \tilde{E}_{\tilde{J}}^{-}\right)$for $\tilde{J} \in \tilde{A}$, we do this so that

$$
\begin{aligned}
\tilde{C}_{J} \cap\left(X_{\text {an }} \times\{0\}\right) & =C_{J} \times\{0\}, & \tilde{U}_{J} \cap V_{J} \times\{0\} & =U_{J} \times\{0\}, \\
\left.\tilde{E}_{J}^{-}\right|_{U_{J} \times\{0\}} & =E_{J}^{-} \times 0, & \tilde{C}_{J^{\prime}} \cap\left(X_{\mathrm{an}} \times\{1\}\right) & =C_{J^{\prime}}^{\prime} \times\{1\}, \\
\tilde{U}_{J^{\prime}} \cap V_{J^{\prime}}^{\prime} \times\{1\} & =U_{J^{\prime}}^{\prime} \times\{1\}, & \left.\tilde{E}_{J^{\prime}}^{-}\right|_{U_{J^{\prime}}^{\prime} \times\{1\}} & =E_{J^{\prime}}^{\prime-} \times 1,
\end{aligned}
$$

whenever $J \in A$ and $J^{\prime} \in A^{\prime}$. This is clearly possible.

Theorem 3.23 constructs a relative Kuranishi atlas $\tilde{\mathcal{K}}$ for $\pi_{\mathbb{C}}: X_{\text {an }} \times \mathbb{C} \rightarrow \mathbb{C}$, of dimension $n+2$. By construction, over $X_{\text {an }} \times\{0\}$ this restricts to the Kuranishi atlas $\mathcal{K}$, and over $X_{\text {an }} \times\{1\}$ it restricts to $\mathcal{K}^{\prime}$.

Theorem 3.24 gives a derived manifold $\tilde{\boldsymbol{X}}_{\mathrm{dm}}$ with $\operatorname{vdim} \tilde{\boldsymbol{X}}_{\mathrm{dm}}=n+2$ and topological space $X_{\mathrm{an}} \times \mathbb{C}$, with a morphism $\tilde{\boldsymbol{\pi}}_{\mathrm{dm}}: \tilde{\boldsymbol{X}}_{\mathrm{dm}} \rightarrow \mathbb{C}$. From Theorem 3.24 (iii) we see that $\tilde{\boldsymbol{X}}_{\mathrm{d} \tilde{\boldsymbol{X}}_{\mathrm{m}}^{0}}^{0}=\tilde{\boldsymbol{\pi}}_{\mathrm{dm}}^{-1}(0) \simeq \boldsymbol{X}_{\mathrm{dm}}$ and $\tilde{\boldsymbol{X}}_{\mathrm{dm}}^{1}=\tilde{\boldsymbol{\pi}}_{\mathrm{dm}}^{-1}(1) \simeq \boldsymbol{X}_{\mathrm{dm}}^{\prime}$.

Now define $\boldsymbol{W}_{\mathrm{dm}}=\tilde{\boldsymbol{X}}_{\mathrm{dm}} \times \tilde{\boldsymbol{\pi}}_{\mathrm{dm}}, \mathbb{C}$,inc $[0,1]$, as a fibre product in the 2-category $\mathbf{d M a n}^{\mathbf{c}}$ of d-manifolds with corners from [18 20], where inc : $[0,1] \hookrightarrow \mathbb{C}$ is the inclusion. By properties of fibre products in $\mathbf{d M a n}^{\mathbf{c}}$ from [18 20], this has topological space $X_{\mathrm{an}} \times[0,1]$ and $\operatorname{vdim} \boldsymbol{W}_{\mathrm{dm}}=n+1$, and boundary

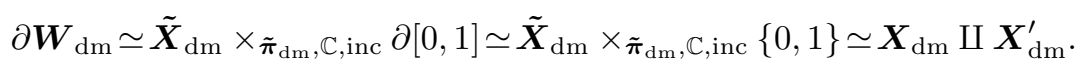

This proves the first part of Proposition 3.18 
For the last part, orientations on $\left(\boldsymbol{X}, \omega_{\boldsymbol{X}}^{*}\right)$ correspond naturally to orientations for $\tilde{\boldsymbol{\pi}}: \tilde{\boldsymbol{X}} \rightarrow Z, \omega_{\tilde{\boldsymbol{X}} / Z}$, by pullback along $\tilde{\boldsymbol{X}} \rightarrow \boldsymbol{X}$, and these correspond to orientations on $\tilde{\boldsymbol{X}}_{\mathrm{dm}}$ by Proposition [3.26, and thus (using oriented fibre products) to orientations on $\boldsymbol{W}_{\mathrm{dm}}$. Since $\partial[0,1]=-\{0\} \amalg\{1\}$ in oriented manifolds, we see that as in (6.15) that $\partial \boldsymbol{W}_{\mathrm{dm}} \simeq-\boldsymbol{X}_{\mathrm{dm}} \amalg \boldsymbol{X}_{\mathrm{dm}}^{\prime}$ in oriented derived manifolds. This completes the proof.

\section{References}

[1] K. Behrend and B. Fantechi, The intrinsic normal cone, Invent. Math. 128 (1997), 45-88.

[2] O. Ben-Bassat, C. Brav, V. Bussi, and D. Joyce, A 'Darboux Theorem' for shifted symplectic structures on derived Artin stacks, with applications, Geometry and Topology 19 (2015), 1287-1359. arXiv:1312.0090.

[3] C. Brav, V. Bussi, D. Dupont, D. Joyce, and B. Szendrői, Symmetries and stabilization for sheaves of vanishing cycles, Journal of Singularities 11 (2015), 85-151. arXiv:1211.3259.

[4] C. Brav, V. Bussi and D. Joyce, A Darboux theorem for schemes with shifted symplectic structure, arXiv:1305.6302, 2013.

[5] V. Bussi, D. Joyce and S. Meinhardt, On motivic vanishing cycles of critical loci, arXiv:1305.6428, 2013.

[6] D. Borisov, Derived manifolds and Kuranishi models,arXiv:1212.1153, 2012.

[7] D. Borisov and J. Noel, Simplicial approach to derived differential geometry, arXiv:1112.0033, 2011.

[8] Y. Cao, Donaldson-Thomas theory for Calabi-Yau four-folds, arXiv:1309.4230, 2013.

[9] Y. Cao and N.C. Leung, Donaldson-Thomas theory for Calabi-Yau fourfolds, arXiv:1407.7659, 2014.

[10] Y. Cao and N.C. Leung, Orientability for gauge theories on Calabi-Yau manifolds, arXiv:1502.01141, 2015.

[11] P.E. Conner, Differentiable Periodic Maps, second edition, Springer Lecture Notes in Mathematics 738, Springer, Berlin, 1979.

[12] S.K. Donaldson and P.B. Kronheimer, The Geometry of Four-Manifolds, OUP, 1990.

[13] S.K. Donaldson and R.P. Thomas, Gauge Theory in higher dimensions, §3 in S.A. Huggett et al., editors, The Geometric Universe, OUP, 1998.

[14] K. Fukaya, Y.-G. Oh, H. Ohta and K. Ono, Lagrangian intersection Floer theory - anomaly and obstruction, I \& II. AMS/International Press, 2009. 
[15] K. Fukaya and K. Ono, Arnold Conjecture and Gromov-Witten invariant, Topology 38 (1999), 933-1048.

[16] R. Hartshorne, Algebraic Geometry, Springer, New York, 1977.

[17] D. Joyce, Algebraic Geometry over $C^{\infty}$-rings, to appear in Memoirs of the A.M.S., 2016. arXiv:1001.0023.

[18] D. Joyce, An introduction to d-manifolds and derived differential geometry, pages 230-281 in L. Brambila-Paz et al., editors, Moduli spaces, LMS Lecture Notes 411, CUP, 2014. arXiv:1206.4207.

[19] D. Joyce, D-manifolds, d-orbifolds and derived differential geometry: a detailed summary, arXiv:1208.4948, 2012.

[20] D. Joyce, D-manifolds and d-orbifolds: a theory of derived differential geometry, to be published by OUP, 2017. Preliminary version (2012) available at http://people.maths.ox.ac.uk/ joyce/dmanifolds.html.

[21] D. Joyce, A classical model for derived critical loci, J. Diff. Geom. 101 (2015), 289-367. arXiv:1304.4508.

[22] D. Joyce, A new definition of Kuranishi space, arXiv:1409.6908, 2014.

[23] D. Joyce, in preparation, 2017.

[24] D. Joyce and Y. Song, A theory of generalized Donaldson-Thomas invariants, Mem. Amer. Math. Soc. 217 (2012), no. 1020. arXiv:0810.5645.

[25] M. Kontsevich and Y. Soibelman, Stability structures, motivic DonaldsonThomas invariants and cluster transformations, arXiv:0811.2435, 2008.

[26] J. Lurie, Derived Algebraic Geometry V, arXiv:0905.0459, 2009.

[27] D. McDuff, Notes on Kuranishi atlases, arXiv:1411.4306, 2014.

[28] D. McDuff and K. Wehrheim, Smooth Kuranishi atlases with trivial isotropy, arXiv:1208.1340, 2012.

[29] J. Milnor, 'On the Steenrod homology theory', pages 101-106 in Collected Papers of John Milnor. IV, AMS, 2009.

[30] T. Pantev, B. Toën, M. Vaquié and G. Vezzosi, Shifted symplectic structures, Publ. Math. I.H.E.S. 117 (2013), 271-328. arXiv:1111.3209.

[31] D.I. Spivak, Derived smooth manifolds, Duke Math. J. 153 (2010), 55-128. arXiv:0810.5174.

[32] R.P. Thomas, A holomorphic Casson invariant for Calabi-Yau 3-folds, and bundles on K3 fibrations, J. Diff. Geom. 54 (2000), 367-438. math.AG/9806111.

[33] B. Toën, Higher and derived stacks: a global overview, pages 435-487 in Algebraic Geometry - Seattle 2005, Proc. Symp. Pure Math. 80, Part 1, AMS, 2009. math.AG/0604504. 
[34] B. Toën, Derived Algebraic Geometry, EMS Surv. Math. Sci. 1 (2014), 153240. arXiv:1401.1044.

[35] B. Toën and G. Vezzosi, Homotopical Algebraic Geometry II, Mem. AMS 193 (2008), no. 902. math.AG/0404373.

Dennis Borisov, Mathematisches Institut, Georg-August Universität Göttingen, Bunsenstrasse 3-5, D-37073 Göttingen, Germany.

E-MAIL: dennis.borisov@gmail.com.

Dominic Joyce, The Mathematical Institute, Radcliffe Observatory QuarTER, WOODSTOCK ROAd, OXFORd, OX2 6GG, U.K.

E-MAIL: joyce@maths.ox.ac.uk. 